\title{
GUIDE TO DIATOMS IN MOUNTAIN LAKES IN THE RETEZAT MOUNTAINS, SOUTH CARPATHIANS, ROMANIA
}

\author{
Krisztina BuczKó \\ Department of Botany, Hungarian Natural History Museum, H-1431 Budapest, Pf. 137, Hungary; \\ buczko@bot.nbmus.hu
}

Buczkó, K. (2016): Guide to diatoms in mountain lakes in the Retezat Mountains, South Carpathians, Romania. - Studia bot. hung. 47(Suppl.): 9-214.

\begin{abstract}
Mountain lakes have special importance in the neo- and palaeolimnology. The diatom flora of mountain regions of Europe has been studied in detail, but less attention was paid to the Romanian part of the Carpathians. Siliceous algae of mountain lakes (1,740-2,122 m a.s.l.) were studied in the Retezat Mts (South Carpathians, Romania). Sediment samples were collected in the deepest points of twenty-three pristine lakes between 2011 and 2014. A total of 152 species and intraspecific diatom taxa were found during this study (excluding the representatives of the two most diverse diatom genera Eunotia and Pinnularia). Altogether 140 taxa were listed and nomenclaturally checked from the previously published results. 79 common species and some rarely reported taxa are illustrated by means of light- (LM) and scanning electron microscope (SEM), and 40 other are presented here only with LM images. A partial diatom checklist is presented with 217 items, 80 of them have been recently recorded for the Retezat Mountains. This compilation focuses on small celled $(<20 \mu \mathrm{m})$ diatoms. The main aim is to help the identification to the hardly visible, lightly silicificied and recently described forms, instead of repeating the earlier published data. This guide consist of a detailed light and scanning electron microscopy documentation of the diatom flora, based on 752 LM and 188 SEM pictures. New Humidophila and Staurosira taxa are presented and a new combination is proposed for Achnanthes helvetica var. minor. The detailed, illustrated guide hopefully will be a useful manual for ecological and palaeoecological surveys in the future.
\end{abstract}

Key words: Mountain lakes, diatoms, oligotrophy, taxonomy

\section{INTRODUCTION}

Diatoms are the most often used algal group in environmental assessments, due to their diversity, abundance and variability, as well as their solid valves (BATTARBEE 1986). Currently, more than 24,000 diatom species have valid scientific names (Fourtanier and Kociolek 2009a, b). MANn and Droop (1996) conservatively estimated that there are 200,000 diatom species, but many of these have only been illustrated in the literature with light microscopy. The study of diatoms is uneven, the pristine, arctic and mountain regions have been paid more attention only in the last couple of years (e.g. CATALAN et al. 2013). 
The diatom flora of mountain regions in East-central Europe has been studied in detail e.g. in the Tatra Mountains (e.g. KaWECKa 2012, Wojtal 2013), but less attention was paid to the Romanian part of the Carpathians. The alga flora of Romania is quite up-to-date thanks to the detailed compilation of CARAUS (2012), who refreshes the Romanian algae list regularly. According to the Caraus' summary, during the last decades of the 20th century, the knowledge on the algal flora of Romania has considerably developed. A lot of research projects were carried out by the scientists from Cluj-Napoca, dealing especially with algae from mountain areas, peat bogs, and other wet habitats, mainly from Transylvania. The first record about the diatom flora of the Retezat Mts was published by PÉTERFI and NAGY-TótH (1963), and soon after several papers followed (PÉTERFI 1966, $1967,1974 a, b)$. The first attempt for diatom based palaeoenvironmental reconstruction with detailed taxa list was published from the Retezat Mountains by PÉTERFi (1974b). In this foretime study Péterfi revealed the main diatom assemblages zones in Lake Zanoguta, taking into consideration of their $\mathrm{pH}$ and life form preferences. Data were interpreted with previously published palynological results and lithostratigraphy, so serves as the first multi-proxy study about the Retezat Mountains. A summary of the algal flora of the Retezat Mountains was presented in 1993 by Péterfi. In this compilation the author summarised all previously published algological data, listing 285 diatom names (PÉTERFI 1993).

My investigations on the siliceous algae of the Retezat Mountains extend back to 2008. The first results focused on diatom-based evidence for abrupt climate changes during the Late Glacial (BuCz Kó et al. 2009), and later the responses of diatoms to the Younger Dryas climatic reversal were presented (BuczKó et al. 2012). A detailed and richly illustrated overview was published on the representatives of the most abundant genus, Aulacoseira (BuczKó et al. 2010), and later data on some rare taxa belonging to the Planothidium and Nupela genera inhabiting glacial lakes of the Retezat Mountains were published (BUCzKó et al. 2013b,c).

Another group of siliceous algae belongs to the crysophytes. The members of Chrysophyceae and Synurophyceae, two chrysophyte classes often produce resting spores that are preserved in the lacustrine sediments. Chrysophyte stomatocysts of the Retezat Mts were studied by SoróczKi-Pintér et al. (2012, 2013). As a first result we reported 83 stomatocyst forms from Lake Gales, from which seven proved to be new for science (SORÓCZKI-PInTÉR et al. 2014).

This compilation focuses on small-celled diatoms. The main aim is to contribute to the hardly visible, lightly silicificied and recently described forms, instead of repeating the earlier published data. The genus Pinnularia is one of the most species rich groups in mountain environment, especially in mires. However, all the representatives of the genus are well visible and they can be satisfactorily studied by means of light microscope therefore the whole genus is excluded from 
this compilation. Nevertheless, the Romanian Pinnularia taxa are under revision (Szigyártó I. L., pers. comm.).

The Eunotia taxa are also missing from this review. This diverse genus needs revision in the light of a recently published monograph (LANGE-BERTALOT et al. 2011).

The aim of this paper is to contribute towards the better understanding of diatom diversity in a poorly investigated part of the Carpathians. The detailed, illustrated guide hopefully will be a useful manual for ecological and palaeoecological surveys in the future. A further aim of this work is to provide a revised taxon list (checklist) of diatoms from the Retezat Mts as a summary of the already published data. We hope that the detailed light (LM) and scanning electron microscope (SEM) documentation will help gaining a better overview of the oligotraphenic diatoms on a world-wide scale.

\section{MATERIAL AND METHODS}

\section{Study site}

The Retezat Mts (Munții Retezat) are located in the western part of the Southern Carpathians ("Transylvanian Alps"). It is one of the highest massifs in Romania. The highest peak is Peleaga (Vârful Peleaga, 2,509 m a.s.l.). The mountain has a versatile alpine and subalpine landscape with alpine meadows, plateaus, rocky ridges, cirque valleys and numerous mountain lakes. Most of them are situated at elevations between 1,900 and 2,200 $\mathrm{m}$ a.s.l. in the cirque valleys. Fifty-eight permanent and forty temporary glacial lakes are known (JANCSIK 2007). The climate is typically alpine: the mean annual temperature in $6{ }^{\circ} \mathrm{C}$, while at the peaks $-2{ }^{\circ} \mathrm{C}$. The coldest month is January (under $-10^{\circ} \mathrm{C}$ mean temperature), while the warmest month is June (around $6{ }^{\circ} \mathrm{C}$ mean temperature). In addition, this is the wettest part of the Eastern and Southern Carpathians, with the highest amount of rainfall in June and the lowest in October (JANCSIK 2007). The mountain comprises granite and granodiorite blocks, which are embedded in crystal slates (JANCSIK 2007).

\section{Field work}

Altogether twenty-three lakes were chosen for detailed analyses. Four collecting campaigns were carried out between 2011 and 2014 to the Retezat Mts by Csilla Kövér as a part of her PhD study (Kövér 2016) (Fig. 1, Table 1). Samples were collected from the deepest points of the lakes using a belly-boat. Chemical composition of sediments was determined (Kövér 2016). We also used some samples of sediment cores of PROLONG project (MAGYARI et al. 2016). Four 
lakes were chosen for multi-proxy palaeolimnological survey in the frame of PROLONG project, namely Brazi, Lake Gales, Lake Lia and Lake Bucura (MAgYARI et al. 2016).

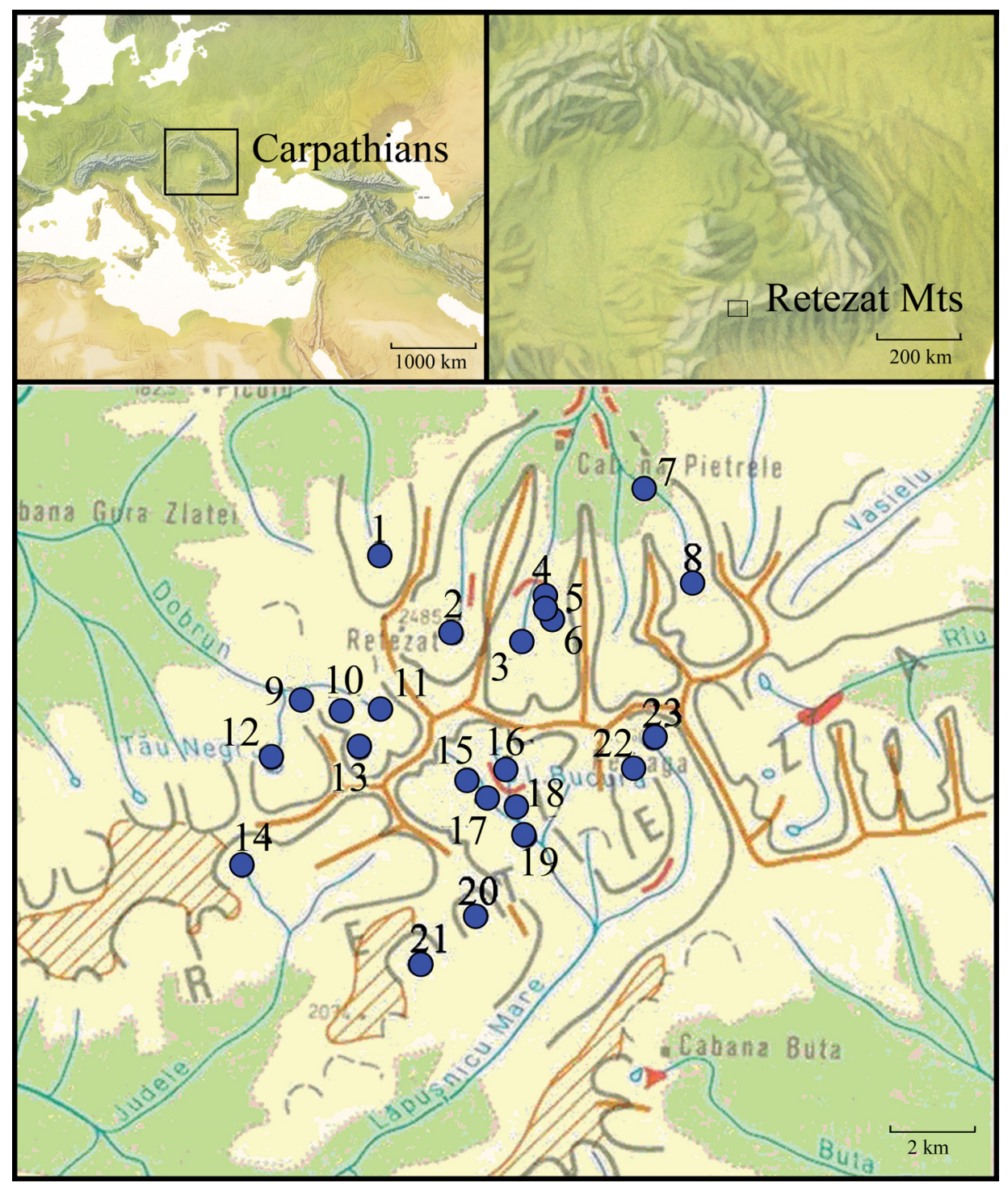

Fig. 1. Locations of the studied lakes in the Retezat Mountains, Romania (see Table 1). $1=$ Stevia, $2=$ Stanisoara, $3=$ Pietrele, $4=$ Pietrelicele-1, $5=$ Pietrelicele-3, $6=$ Pietrelicele- $2,7=$ Brazi, $8=$ Gales, $9=$ Gemenele, $10=$ Stirbu, $11=$ Caprelor, $12=$ Negru, $13=$ Lezilor, $14=$ Zanoaga, $15=$ Viorica, $16=$ Bucura, $17=$ Florica, $18=$ Ana, $19=$ Lia, $20=$ Slaveiu, $21=$ Turcelu, $22=$ Peleguta, 23 = Peleaga (partly after KövÉR 2016). 
Table 1. Details of the studied lakes in the Retezat Mts with surface area and the water depth at deepest points (after KövÉR 2016)

\begin{tabular}{|c|c|c|c|c|c|}
\hline \multirow[t]{2}{*}{ Lakes } & \multicolumn{2}{|c|}{ Coordinates } & \multirow{2}{*}{$\begin{array}{l}\text { Altitude } \\
\text { a.s.l. (m) }\end{array}$} & \multirow{2}{*}{$\begin{array}{l}\text { Surface } \\
\text { (ha) }\end{array}$} & \multirow{2}{*}{$\begin{array}{c}\text { Water } \\
\text { depths }(m)\end{array}$} \\
\hline & $\mathrm{N}\left({ }^{\circ}\right)$ & $\mathrm{E}\left({ }^{\circ}\right)$ & & & \\
\hline Stevia (1) & 45.384083 & 22.844833 & 2,060 & 0.48 & 10.6 \\
\hline Stanisoara (2) & 45.375100 & 22.861967 & 1,990 & 0.76 & 0.45 \\
\hline Pietrele (3) & 45.375100 & 22.872350 & 1,995 & 0.38 & 0.55 \\
\hline Pietrelicele (4) & 45.376150 & 22.877167 & 1,950 & 0.08 & 2.2 \\
\hline Pietrelicele-3 (5) & 45.375117 & 22.883483 & 1,989 & 0.09 & 1.8 \\
\hline Pietrelicele-2 (6) & 45.375317 & 22.881933 & 2,007 & 0.22 & 2.2 \\
\hline Brazi (7) & 45.396567 & 22.901591 & 1,740 & 0.21 & 0.9 \\
\hline Gales (8) & 45.385611 & 22.909133 & 1,990 & 3.69 & 15.5 \\
\hline Gemenele (9) & 45.365417 & 22.839111 & 1,920 & 2.48 & 6.2 \\
\hline Stirbu (10) & 45.362722 & 22.853111 & 2,088 & 1.05 & 9.0 \\
\hline Caprelor (11) & 45.359583 & 22.847639 & 2,135 & 0.20 & 1.2 \\
\hline Negru (12) & 45.359222 & 22.826833 & 2,036 & 4.32 & 27.7 \\
\hline Lezilor (13) & 45.360917 & 22.843694 & 2,092 & 0.14 & 2.1 \\
\hline Zanoaga (14) & 45.346038 & 22.822337 & 1,998 & 6.63 & 30.5 \\
\hline Viorica (15) & 45.358082 & 22.863482 & 2,063 & 0.75 & 6.1 \\
\hline Bucura (16) & 45.361516 & 22.874340 & 2,040 & 8.53 & 16.5 \\
\hline Florica (17) & 45.358399 & 22.861342 & 2,085 & 0.51 & 1.4 \\
\hline Ana (18) & 45.356617 & 22.868467 & 1,976 & 3.49 & 11.9 \\
\hline Lia (19) & 45.352402 & 22.877247 & 1,910 & 1.26 & 4.0 \\
\hline Slaveiu (20) & 45.339333 & 22.874583 & 1,930 & 3.39 & 10.6 \\
\hline Turcelu (21) & 45.333222 & 22.858222 & 2,020 & 0.20 & 0.4 \\
\hline Peleguta (22) & 45.358028 & 22.898611 & 2,097 & 0.86 & 5.6 \\
\hline Peleaga (23) & 45.364417 & 22.902111 & 2,122 & 2.05 & 4.1 \\
\hline
\end{tabular}

Preparation - counting procedure

For analyses of the siliceous algae, samples were prepared by standard digestion procedures (BATTARBEE 1986). Aliquot-evaporated suspensions were embedded in Zrax and Pleurax. From each sample at least 400 valves were counted using a light microscope (Leica DM LB2 equipped with 100 HCX PLAN APO objective and Fujifilm FinePix S2 Pro Digital Camera; later VSI-3.OM(H) digital camera). During the counting procedure images of ambiguous valves were taken; later the images were arranged according to the diatom genera to which they belong, and the small forms were grouped together. For SEM cleaned samples were air-dried on an aluminium stub. Specimens were coated with gold-palladium using a XC-7620 Mini Sputter Coater for $120 \mathrm{~s}$ at $16 \mathrm{~mA}$, and studied with a Hitachi S-2600N scanning electron microscope 
operated at $20 \mathrm{kV}$ and 5-8 mm distance. Taxonomy follows Krammer and LANGe-Bertalot (1991-2004), and Siver et al. (2005). Some more recent books (e.g. Diatoms of Europe, Iconographia Diatomologica, Bibliotheca Diatomologica floras) and papers (esp. Diatom Research papers) were also used for identification purposes. During the assembly of the diatom flora of the Retezat Mountains names were listed from the monograph of PÉTERFI (1993), nomenclature was checked and updated according to AlgaeBase (GUIRY and GUIRY 2016), accessed between 2-25 April 2016.

Abundance and constancy of taxa were calculated as in STENGER-KovÁcs and LENGYEL (2015).

\section{RESULTS}

A total of 152 species and intraspecific diatom taxa were found during this study (excluding the representatives of genera Eunotia and Pinnularia). Altogether 140 taxa were listed and nomenclaturally checked from the previously published results (PÉTERFI 1993). The merged list of taxa consists altogether of 217 diatoms. Below, 79 common species and some rarely reported taxa are illustrated by means of LM and SEM, and 40 other are presented here only with LM images. Taxa have been found in our recent study are marked with an asterisk $\left(^{*}\right)$. Taxa reported in PÉTERFi (1993) are marked with a hash mark (\#). An asterisk and a hash mark (*\#) can be found before the name of taxa that were found in both compilations.

\section{ENUMERATION}

*Achnanthes didyma Hustedt 1933: 405, fig. 857. - (Plate 84: Fig. 1) References: Hustedt (1927, 1933), Krammer and LANGe-Bertalot (2004). - Remark: This species was only found in Lake Lia, rare.

*\#Achnanthes oblongella - (see pp. 28-29, Plate 1).

*\#Achnanthes trinodis (Ralfs) Grunow in Van Heurck 1880, expl. pl. XXVII: fig. 50. - (Plate 84: Fig. 2) - References: VAN HeurCK (1880), Krammer and LANGe-Bertalot (1991). - Remarks: PÉTERFi (1993) reported this taxon from running waters. In our recent study only a few valves were found in Lake Stevia (less than $1 \%$ ).

*Achnanthes ventralis (Krasske) Lange-Bertalot in Lange-Bertalot et Krammer 1989: 155, fig. 79: 22-33, fig. 46: 7, fig. 47: 1-4. - References: LANGE-BERTALOT and Krammer (1989), Lange-Bertalot and Metzeltin (1996), Hofmann $e t$ al. (2013) as Psammothidium ventrale. - Remarks: In our recent study we found it in Lake Lezilor, Stevia, Stirbu, Turcelu and Viorica. Never was abundant (less than 1\%).

*Achnanthidium bioretii (H. Germain) Monnier, Lange-Bertalot et Ector in Monnier, Lange-Bertalot, Hoffmann et Ector 2007: 155. - (Plate 84: Figs 3-5) References: Monnier et al. (2007), Krammer and LANGe-Bertalot (1991), 
Hofmann et al. (2013) as Psammothodium bioretii. Remark: Rare, it was found in the Lake Caprelor.

\#Achnanthidium kryophila (J. B. Petersen) Bukhtiyarova 1995: 420. Reference: Bukhtiyarova (1995). - Remark: PÉterfi (1993) reported this species as Achnanthes kryophila from mires and running waters.

*\#Achnanthidium lineare W. Smith 1855: 8, pl. I: fig. 9. - (Plate 84: Figs 6-8) - References: Smith (1855), VAN DE Vijver et al. (2011). - Remarks: PÉTERfi (1993) published it as Achnanthes linearis from running waters. In our recent study was found it in Lake Lia and Peleaga, never exceeded 1\% in relative abundance.

*\#Achnanthidium minutissimum - (see pp. 30-31, Plate 2).

*\#Adlafia bryophila (Petersen) Gerd Moser, Lange-Bertalot et Metzeltin 1998: 89. - References: Moser et al. (1998), Hofmann et al. (2013). - Remarks: PÉTERFI (1993) found it in running waters, and reported it as Navicula bryophila. Very rare in our recent study, only two valves were found in the Lake Turcelu and Lake Gales.

\#Adlafia minuscula (Grunow) Lange-Bertalot in Lange-Bertalot et Genkal 1999: 32. - References: LANGe-Bertalot and Genkal (1999), HofmANn et al. (2013). - Remarks: PÉTERFI (1993) found it in running waters and reported it as Navicula minuscula. It is probably conspecific with Navicula importuna according to KRAMMER and LANGE-BERTALOT (1999a: 207).

\#Amphipleura pellucida (Kützing) Kützing 1844: 103, pl. 3: fig. 52, pl. 30: fig. 84. - References: KÜTZING (1844), HofMANN et al. (2013). - Remark: Only PÉTERFI (1993) published this species from running waters.

* Amphora copulata - (see pp. 32-33, Plate 3).

* Amphora inariensis - (see pp. 34-35, Plate 4).

*Amphora indistincta Levkov 2009: 69, 287, pl. 56: figs 20-21, pl. 78: figs 29-39, pl. 152: fig. 3, pl. 193: figs 1-6, pl. 196: fig. 3. - References: LeVKov (2009), HofmANn et al. (2013). - Remark: Rare in the sediment of Lake Lia.

*\#Amphora pediculus (Kützing) Grunow ex A. Schmidt 1875: pl. 26: fig. 99. - References: Schmidt (1875b), Levkov (2009), HofmANn et al. (2013). Remarks: PÉTERFi (1993) reported this species from lakes and running waters. Rare, it was found in Lake Lia in our recent study.

*Asterionella formosa Hassall 1850: 9-10, pl. II: fig. 5 (p. 62). - (Plate 84: Fig. 11) - References: Hassall (1850), Hofmann et al. (2013). - Remark: Rare, it was found only in Lake Lezilor and Negru in our recent study less than $1 \%$.

* Aulacoseira alpigena - (see pp. 36-37, Plate 5).

*Aulacoseira ambigua - (see pp. 38-39, Plate 6).

*\#Aulacoseira granulata (Ehrenberg) Simonsen 1979: 58. - References: SIMONSEN (1979), Houk (2003). - Remarks: PÉTERfi (1993) reported this species from lakes and running waters as Melosira granulata. In our recent study only a few valves were found in Lake Pietrelice- 2 . 
\#Aulacoseira islandica (Otto Müller) Simonsen 1979: 60. - References: SIMONSEN (1979), Houk (2003). - Remark: In PÉTERFi (1993) as Melosira islandica subsp. islandica reported from glacial lakes.

\#Aulacoseira italica (Ehrenberg) Simonsen 1979: 60. - References: SimONSEN (1979), HouK (2003). - Remark: It is reported from running waters as Melosira italica (PÉTERFi 1993).

*Aulacoseira laevissima - (see pp. 40-41, Plate 7).

\#Aulacoseira lirata (Ehrenberg) R. Ross in Hartley, Ross et D. M. Williams 1986: 606. - References: HARTLEY et al. (1986), Houk (2003). - Remark: This species is reported as Melosira distans var. lirata f. seriata from glacial lakes (PÉTERFI 1993).

*Aulacoseira nivalis - (see pp. 42-43, Plate 8).

*Aulacoseira perglabra - (see pp. 44-45, Plate 9).

*Aulacoseira pfaffiana - (see pp. 46-47, Plate 10).

\#Aulacoseira subarctica (Otto Müller) E. Y. Haworth 1990: 195. - References: HaworTH (1990), Houk (2003). - Remark: This species is reported as Melosira italica var. subarctica, and was found only in glacial lakes (PÉTERFI 1993).

* Aulacoseira valida - (see pp. 48-49, Plate 11).

*\#Boreozonacola bustedtii - (see pp. 50-51, Plate 12).

*\#Brachysira brébissonii - (see pp. 52-53, Plate 13).

*\#aloneis silicula (Ehrenberg) Cleve 1894: 51. - References: Cleve (1894). Krammer and LANge-Bertalot (1999a), HofmAnn et al. (2013). Remarks: It is reported as Caloneis ventricosa from running waters (PÉTERfi 1993). Only a few valves were found in Lake Viorica in our recent study.

*\#Cavinula cocconeiformis (W. Gregory ex Greville) D. G. Mann et A. J. Stickle in Round, Crawford et D. G. Mann 1990: 665. - (Plate 84: Fig. 12) - References: Round et al. (1990) LANGe-Bertalot and Metzeltin (1996), Hofmann et al. (2013). - Remarks: PÉTERFI (1993) recorded it from running waters. In our recent study only one valve was found in Lake Lia.

* Cavinula pseudoscutiformis - (see pp. 54-55, Plate 14).

*Chamaepinnularia mediocris (Krasske) Lange-Bertalot et Krammer in Lange-Bertalot et Metzeltin 1996: 35, pl. 28: figs 48-51. - (Plate 84: Fig. 13) - References: Lange-Bertalot and Metzeltin (1996), Hofmann et al. (2013). - Remark: Very rare, only a few valves were found in the Lake Bucura.

*Chamaepinnularia soebrensis (Krasske) Lange-Bertalot et Krammer in Lange-Bertalot et Metzeltin 1996: 36, pl. 28: figs 52-55. - (Plate 84: Fig. 14) - References: Lange-Bertalot and Metzeltin (1996), Hofmann et al. (2013). - Remark: Very rare, only a few valves were found in Lake Bucura.

*\#Cocconeis disculus (Schumann) Cleve in Cleve et Jentzsch 1882: 139. - (Plate 84: Fig. 15) - References: Cleve and Jentzsch (1882), HofmAnN et al. (2013). Remarks: PÉTERFI (1993): in running water. Only one valve was detected in Lake Lia. 
\#Cocconeis placentula var. euglypta (Ehrenberg) Grunow 1884: 110. Reference: GRUNOW (1884). - Remark: PÉTERFI (1993) recorded this variety from running waters.

\#Cocconeis placentula var. intermedia (Héribaud-Joseph et M. Peragallo) Cleve 1895. - Reference: Cleve (1895). - Remark: PÉterfi (1993) recorded this variety from running waters.

${ }^{*}$ Craticula cf. submolesta - (see pp. 56-57, Plate 15).

\#Cymbella affinis Kützing 1844: 80, pl. 6: fig. 15. - Reference: Kü TZING (1844). - Remarks: According to AlgaeBase catalogue Cymbella tumidula is currently regarded as a taxonomic synonym of C. affinis (GUIRY and GUIRY 2016). The taxonomic position of this species is uncertain. PÉTERFI (1993) published this data from lakes and running waters as C. tumidula.

\#Cymbella aspera (Ehrenberg) Cleve 1894: 175. - References: Cleve (1894), Krammer (2002). - Remark: PÉterfi (1993) published this data from lakes and running waters.

\#Cymbella bistritzae Oltean et Zanoschi 1963: 183, fig. 1. - Reference: OlteAn and Zanoschi (1963). - Remark: This species is reported from mires only by PÉTERFI (1993).

\#Cymbella belvetica var. punctata Hustedt - Remark: PÉTERFI (1993) reported it from running water of the Retezat Mts.

\#Cymbella laevis Nägeli in Rabenhorst 1863:21. - Reference: RABENHORST (1863). - Remark: PÉTERFI (1993) published this data from mires.

\#Cymbella neocistula Krammer 2002: 94, 169, pl. 85: figs 1-4; pl. 86: figs 1-7; pl. 87: figs 1-9; pl. 88: figs 1-8; pl. 89: figs 1-7; pl. 90: figs 1-8; pl. 91: figs 1-6; pl. 92: figs 1-3; pl. 93: figs 1-5. - Reference: KRAMMER (2002). - Remark: Only PÉTERFI (1993) found this species in running waters.

\#Cymbella ventricosa var. hankensis Skv. - Remarks: Only PÉTERFI (1993) mentioned this variety. Uncertain taxon.

\#Cymbella ventricosa var. ovata Grunow - Remarks: PÉTERFI (1993) reported this variety from running waters. Uncertain taxon.

*\#Cymbopleura amphicephala (Nägeli) Krammer 2003: 70, pl. 91: figs 1-18; pl. 93: figs 2-8. - References: KRAMmeR (2003), HofmANn et al. (2013). - Remarks: PÉTERFi (1993) reported this species from lakes as Cymbella amphicephala. Not rare in Lake Brazi, Lia and Gales, Turcelus and Zanoaga.

\#Cymbopleura cuspidata (Kützing) Krammer 2003: 8, pl. 1: figs 1-12; pl. 2: figs 1-11; pl. 6: figs 5-8. - Reference: KRAMMER (2003). - Remark: PÉTERFI (1993) reported this species from lakes as Cymbella cuspidata.

*Cymbopleura apiculata - (see pp. 58-59, Plate 16).

*\#Cymbopleura naviculiformis - (see pp. 60-61, Plate 17). 
*Diadesmis contenta var. biceps (Grunow) P. B. Hamilton in Hamilton, Poulin, Charles et Angell 1992: 30 (table 4). - (Plate 84: Fig. 16) - Reference: Hamilton et al. (1992). - Remark: Only one valve was found in Lake Gemenele in our recent study.

\#Diatoma ehrenbergii Kützing 1844: 48, pl. 17: fig. 17 (1-3). - Reference: KÜtZING (1844). - Remark: PÉTERFI (1993) reported it from running waters as Diatoma vulgaris var. ehrenbergii.

\#Diatoma ehrenbergii f. capitulata (Grunow) Lange-Bertalot 1993: 22, pl. 3: figs 4-14. - Reference: LANGE-BERTALOT (1993). - Remark: In running waters (PÉTERFI 1993).

*\#Diatoma hyemalis (Roth) Heiberg 1863: 58. - (Plate 84: Fig. 10) - References: Heiberg (1863), Krammer and Lange-Bertalot (2000), Hofmann et al. (2013). - Remarks: PÉTERFI (1993) reported this species from lakes and running waters. We found it in lake Bucura, Lia, Pietrelice-2 and Viorica. Rare.

*\#Diatoma mesodon - (see pp. 62-63, Plate 18).

\#Diatoma vulgaris Bory 1824: 461, fig. 1. - Reference: BORY DE SAINTVinCENT (1824). - Remark: PÉTERFi (1993) reported it from mires.

\#Diatoma vulgaris var. linearis Grunow in Van Heurck 1881: pl. 50: figs 7-8. - Reference: VAN HeurCK (1881). - Remark: PÉTERFI (1993) reported it from running waters as Diatoma vulgare var. lineare.

\#Diatoma vulgaris var. producta Grunow 1862: 363. - Reference: GRUNOW (1862). - Remark: PÉTERFI (1993) reported it from running waters as Diatoma vulgare var. productum.

\#Diploneis oblongella (Nägeli ex Kützing) Cleve-Euler 1922: 57. - Reference: Cleve-Euler (1922). - Remark: PÉTerfi (1993) reported this species from running waters.

*Encyonema caespitosum Kützing 1849: 61. - References: KÜTZING (1849), HofmANN et al. (2013). - Remark: It was rarely found in Lake Gemenele.

*\#Encyonema elginense (Krammer) D. G. Mann in Round, R. M. Crawford et D. G. Mann 1990: 666. - References: Round et al. (1990), Hofmann et al. (2013). - Remarks: PÉTERFi (1993) reported as Cymbella turgida currently regarded as a taxonomic synonym of Encyonema elginense. He found it in mires, lakes and running waters. In our recent study this species was found in Lake Gemenele.

*Encyonema gaeumannii - (see pp. 64-65, Plate 19).

*Encyonema bebridicum Grunow ex Cleve 1891: 48, pl. II: figs 16-17. - (Plate 84: Fig. 21) - Reference: CLEVE (1891). - Remark: This species is not rare in our recent study, it was found in Lake Brazi, Gemenele, Pietrele, Pietrelice- 2 and Slavieau.

*\#Encyonema minutum (Hilse) D. G. Mann in Round, R. M. Crawford et D. G. Mann 1990: 667. - References: Round et al. (1990), Krammer (1997), HofmanN et al. (2013). - Remarks: Not rare in our recent study, was found in 
Lake Ana, Brazi, Bucura, Florica, Lezilor, Lia, Peleguta, Pietrele, Pietrelice 1, Pietrelice 2, Pietrelice 3, Viorica. Its constancy is $4(61 \%)$ in our recent study.

*\#Encyonema neogracile - (see pp. 66-67, Plate 20).

*\#ncyonema perpusillum - (see pp. 68-69, Plate 21).

\#Encyonema prostratum (Berkeley) Kützing 1844: 82, pl. 25: fig. 7. - References: KÜTZING (1844), KRAMMER (1997). - Remark: PÉTERFI (1993) reported it from running waters as Cymbella prostrata.

*Encyonema silesiacum - (see pp. 70-71, Plate 22).

\#Encyonema ventricosum (C. Agardh) Grunow in A. Schmidt et al. 1875: pl. 10: fig. 59. - References: SCHMidt (1875a), KRAMmer (1997). - Remarks: PÉTERFi (1993) reported it from mires, lakes and running waters as Cymbella ventricosa. Two varieties of $C$. ventricosa (var. hankensis and var. ovata) were also reported by him.

\#Encyonopsis cesatii (Rabenhorst) Krammer 1997: 152, 156, pl. 182: figs 1-13; pl. 183: figs 10-12; pl. 185: figs 1-7, 11-13. - References: KRAMMER (1997), Hofmann et al. (2013). - Remark: PÉterfi (1993) reported it from running waters as Cymbella cesatii.

*Encyonopsis microcephala (Grunow) Krammer 1997: 91, pl. 143: figs 1, 4-5, 8-26; pl. 146: figs 1-5; pl. 47: figs 1-3; pl. 148: figs 4, 7. - Reference: KRAMMER (1997). - Remark: It was only found in Lake Negru in our recent study.

\#Epithemia adnata (Kützing) Brébisson 1838: 16. - Reference: BRÉBISSON (1838). - Remark: PÉTERFI (1993) reported it from running waters as Epithemia zebra.

*Fragilaria brevistriata Grunow in van Heurck 1885: 157, pl. 45: fig. 32. (Plate 84: Fig. 9) - References: VAn Heurck (1885), HofmAnn et al. (2013). - Remark: This species was found in Lake Caprelor, Peleaga, Peleguta and Pietrelice-3.

*\#ragilaria capucina Desmazières 1830: No. 453. - Reference: DesmazIÈRES (1830). - Remarks: PÉTERFI (1993) reported this species from mires and lakes as Fragilaria capucina var. capucina, and from lakes as Fragilaria capucina var. lanceolata. Common in our recent study, we found it in lakes Bucura, Lezilor, Peleaga, Pietrelice-2, Stevia, Turcelu, Viorica, Zanoaga (it reached 35\% in relative abundance).

\#Fragilaria capucina subsp. rumpens (Kützing) Lange-Bertalot 1993: 45, Bacill. 2/3, fig. 108: 16-21, fig. 110: 1-6A. - Reference: LANGE-BERTALOT (1993). - Remark: PÉTERFI (1993) reported it as Synedra rumpens from mires and running waters.

*\#Fragilaria gracilis - (see pp. 72-73, Plate 23).

*Fragilariforma bicapitata - (see pp. 74-75, Plate 24).

*\#ragilariforma virescens - (see pp. 76-77, Plate 25).

\#Fragilariforma virescens var. mesolepta (Rabenhorst) N. A. Andresen, Stoermer et R. G. Kreis, Jr. 2000: 414. - References: ANDRESEN et al. (2000). Remark: PÉTERFI (1993) reported this taxon from running waters as Fragilaria virescens var. mesolepta. 
*\#Frustulia crassinervia - (see pp. 78-79, Plate 26).

\#Frustulia rhomboides (Ehrenberg) De Toni 1891: 277. - Reference: DE TONI (1891). - Remark: PÉTERFI (1993) reported this taxon from running waters. \#Frustulia saxonica Rabenhorst 1853: 50, pl. 7: fig. 1. Alg. Sach. Nr. 42. Reference: RABENHORST (1853). - Remark: PÉTERfi (1993) reported this taxon from lakes and running waters as Frustulia rhomboides var. saxonica.

\#Frustulia vulgaris(Thwaites) De Toni 1891: 280. - Reference: DE Toni (1891). - Remark: PÉTERFI (1993) reported this taxon from lakes and running waters.

\#Geissleria decussis (Østrup) Lange-Bertalot et Metzeltin 1996: 65, pl. 104: fig. 2; pl. 125: figs 3-6. - References: LANGE-BERTALOT and METZELTin (1996), Potapova (2009d). - Remark: PÉterfi (1993) reported this species from running waters as Navicula decussis.

*Geissleria cf. schoenfeldii - (see pp. 80-81, Plate 27).

*Genkalia boreoalpina - (see pp. 82-83, Plate 28).

*\#Genkalia digitulus - (see pp. 84-85, Plate 29).

*Genkalia subprocera - (see pp. 86-87, Plate 30).

\#Gomphonema auritum A. Braun ex Kützing 1849: 68. - Reference: KüTZING (1849). - Remark: PÉTERFI (1993) reported this taxon from lakes and running waters as Gomphonema gracile var. auritum.

*\#Gomphonema clavatum Ehrenberg 1832: 88. - Reference: EHRENBERG (1832). - Remarks: PÉTERFi (1993) reported this taxon from mires, lakes and running waters as Gomphonema longiceps var. subclavatum and G. longiceps var. montanum. G. longiceps var. subclavatum and G. longiceps var. montanum are currently regarded as a taxonomic synonym of G. clavatum. Gomphonema clavatum is conspecific with $G$. olivaceum according to REICHARDT (2015), but not with $G$. clavatum sensu Krammer and Lange-Bertalot (GUIRY and GUIRY 2016). In our recent study it was found in Lake Pelaguta.

*Gomphonema exilissimum (Grunow) Lange-Bertalot et E. Reichardt in Lange-Bertalot et Metzeltin 1996: 70, pl. 62: figs 22-27. - Reference: LANGEBertalot and Metzeltin (1996). - Remark: Rare in our recent study. Lake Lia.

\#Gomphonema gracile Ehrenberg 1838: 217, pl. 18: fig. 3. - Reference: EHRENBERg (1838). - Remark: PÉTERFi (1993) reported this taxon from mires, lakes and running waters.

*Gomphonema hebridense W. Gregory 1854: 99, pl. 4: fig. 19. - (Plate 84: Fig. 20) - Reference: GREgory (1854). - Remark: Rare, it was found in Lake Peleaga in our recent study.

\#Gomphonema intricatum Kützing 1844: 87, pl. 9: fig. 4. - Reference: KÜtZING (1844). - Remark: PÉTERFI (1993) reported this taxon from mires.

\#Gomphonema olivaceum (Hornemann) Brébisson 1838: 14. - Reference: BRÉBISSON (1838). - Remark: PÉterfi (1993) reported this taxon from lakes. 
*Gomphonema pala E. Reichardt 2001: 212, pl. 10: figs 1-22; pl. 11: figs 4-7. - (Plate 84: Fig. 18) - References: Reichardt (2001), HofmAnN et al. (2013). - Remark: It was found only in Lake Peleguta in our recent study.

*\#omphonema parvulum (Kützing) Kützing 1849: 65. - (Plate 84: Fig. 19) - References: KütZING (1849); KRAMMER and LANGe-BeRTAlot (1999a), HofmANn et al. (2013). - Remarks: PÉterfi (1993) reported this taxon from mires, lakes and running waters. Also reported as Gomphonema parvulum var. lagenula that is currently regarded as a taxonomic synonym of Gomphonema parvulum. In our recent study it was found in Lake Ana, Gales, Lia, Pietrelica-2 and Zanoaga. It is the most common Gomphonema species in the Retezat Mts.

\#Gomphonema pumilum (Grunow) E. Reichardt et Lange-Bertalot 1991: 528, pl. 6: figs 4-11. - References: REICHARDT and LANGE-BERTALOT (1991), Hofmann et al. (2013). - Remark: PÉterfi (1993) reported this taxon from running waters as Gomphonema intricatum var. pumilum.

*Hannaea arcus - (see pp. 88-89, Plate 31).

\#Hannaea linearis (Holmboe) Álvarez-Blanco et S. Blanco 2013: 147. Reference: Álvarez-Blanco and Blanco (2013). - Remark: Péterfi (1993) reported this taxon from running waters.

*Hantzschia amphioxys (Ehrenberg) Grunow in Cleve et Grunow 1880: 103. - (Plate 84: Fig. 22) - References: CleVE and GRUNOW (1880), KRAMMER and Lange-Bertalot (1999b), Hofmann et al. (2013). - Remarks: Péterfi (1993) reported this taxon from mires, lakes and running waters. In our recent study it was found in Lake Florica and Pietrelice-1.

*\#Humidophila contenta (Grunow) Lowe, Kociolek, J. R. Johansen, Van de Vijver, Lange-Bertalot et Kopalová 2014: 357. - References: HofMANN et al. (2013) as Diadesmis contenta, Lowe et al. (2014). - Remarks: PÉterfi (1993) reported this taxon from lakes as Navicula contenta. In our recent study it was found in Lake Brazi, Gemenele and Pietrelice-2.

${ }^{*}$ Humidophila fukushimae - (see pp. 90-91, Plate 32).

*\#Humidophila perpusilla (Grunow) Lowe, Kociolek, Johansen, Van de Vijver, Lange-Bertalot et Kopalová 2014: 358. - (Plate 84: Fig. 17) - References: KrAmmer and LANGe-Bertalot (1999a), HofmAnn et al. (2013), Lowe et al. (2014). - Remarks: PÉTERFi (1993) reported this taxon from mires, lakes and running waters as Navicula perpusilla. In our recent study it was found in Lake Bucura, Negru and Viorica. Rare.

*\#umidophila schmassmannii - (see pp. 94-95, Plate 34).

*Humidophila sp. - (see pp. 92-93, Plate 33).

*Kobayasiella parasubtilissima (H. Kobayasi et T. Nagumo) Lange-Bertalot 1999: 268. - (Plate 84: Figs 23-24) - References: LANGE-BERTALOT (1999b), HofMANN et al. (2013). - Remarks: This species was found in Lake Turcelu. Rare taxon. 
\#Kobayasiella subtilissima (Cleve) Lange-Bertalot 1999: 268. - Reference: LANGe-Bertalot (1999b). - Remark: PÉTERFi (1993) reported this taxon from mires and lakes as Navicula subtilissima.

\#Kurtkrammeria neoamphioxys (Krammer) L. Bahls 2015: 176. - Reference: BAHLs (2015). - Remark: PÉTERFI (1993) reported this taxon from mires and lakes as Cymbella amphioxys.

\#Lindavia ocellata (Pantocsek) T. Nakov et al. 2015: 256. - Reference: NA Kov et al. (2015). - Remark: PÉTERFi (1993) reported this taxon from mires, lakes and running waters as Cyclotella ocellata.

\#Luticola mutica (Kützing) D. G. Mann in Round, R. M. Crawford et D. G. Mann 1990: 670. - Reference: Round et al. (1990). - Remark: PÉTERFi (1993) reported this taxon from mires, lakes and running waters probably as Navicula mutica, $N$. vanheurckii and $N$. rotaeana.

\#Martyana martyi (Héribaud-Joseph) Round in Round, Crawford et D. G. Mann 1990: 673. - Reference: Round et al. (1990). - Remark: PÉteRfi (1993) reported this taxon from running waters as Opephora martyi.

*\#Meridion circulare - (see pp. 96-97, Plate 35).

*\#Meridion circulare var. constrictum - (see pp. 98-99, Plate 36).

*Microcostatus krasskei - (see pp. 100-101, Plate 37).

*Microfissurata paludosa - (see pp. 102-103, Plate 38).

*\#Navicula angusta Grunow 1860: 528, pl. 3: fig. 19. - (Plate 85: Fig. 1) References: GRunow (1860), HofmANn et al. (2013). - Remarks: PÉterfi (1993) reported this taxon from mires. In our recent study we found it in Lake Negru.

*\#Navicula capitatoradiata H. Germain 1981: 188, pl. 72: figs 7, 7bis. References: Germain (1981), Hofmann et al. (2013). - Remarks: Péterfi (1993) reported this taxon from lakes. Also as N. cryptocephala var. intermedia. Only in the Lake Pietrele. Rare.

*\#Navicula cryptocephala - (see pp. 104-105, Plate 39).

*Navicula cryptotenella Lange-Bertalot 1985: 62, figs 18, 22-23. - (Plate 85: Figs 2-3) - References: KrAMmer and LANGE-BERTALOT (1999a), LANGEBertalot (2001), Hofmann et al. (2013). - Remark: Rare, it was found in the Lake Viorica in our recent study.

*Navicula detenta Hustedt 1943: 164, fig. 31. - (Plate 85: Figs 4-5) References: Hustedt (1943), HofmanN et al. (2013). - Remark: In our recent study was found only a few valves in Lake Peleguta.

\#Navicula boefleri Cholnoky in Cholnoky et Schindler 1953: 607, figs 34-37. - Reference: Cholnoky and Schindler (1953). - Remark: PÉterfi (1993) reported this taxon from mires.

\#Navicula ingrata Krasske 1938: 528, pl. 11: figs 17-18. - Reference: KRASS Ke (1938). - Remark: PÉTERFI (1993) reported this taxon from lakes. 
\#Navicula kotschyi Grunow 1860: 538, pl. 2: fig. 12. - Reference: GRUNOW (1860). - Remark: PÉTERFI (1993) reported this taxon from running waters.

\#Navicula radiosa Kützing 1844: 91, pl. 4: fig. 23. - References: KüTZING (1844), HofmAnn et al. (2013). - Remark: PÉTERfi (1993) reported this taxon from running waters.

*\#Navicula rhynchocephala - (see pp. 106-107, Plate 40).

\#Neidium affine (Ehrenberg) Pfitzer 1871: 39. - Reference: Pfitzer (1871). - Remark: PÉTERFI (1993) reported this taxon from lakes.

\#Neidium affine var. ceylonicum (Skvortsov) Reimer 1959: 11, pl. 1: fig. 7 (as 'ceylonica') - Reference: REIMER (1959). - Remark: PÉTERfi (1993) reported this taxon from running waters.

*\#Neidium affine var. longiceps - (see pp. 108-109, Plate 41).

*Neidium alpinum - (see pp. 110-111, Plate 42).

*\#Neidium amphigomphus - (see pp. 112-113, Plate 43).

*\#Neidium ampliatum - (see pp. 114-115, Plate 44).

*Neidium cf. antarcticum - (see pp. 116-117, Plate 45).

*Neidium bisulcatum - (see pp. 118-119, Plate 46).

\#Neidium bisulcatum var. subundulatum (Grunow) Reimer in Patrick et Reimer 1966: 398, pl. 36: figs 7-8. - Reference: PATrick and Reimer (1966). - Remark: PÉterfi (1993) reported this taxon from lakes and running waters.

*\#Neidium continentale - (see pp. 120-121, Plate 47).

*\#Neidium iridis - (see pp. 122-123, Plate 48).

\#Neidium productum (W. Smith) Cleve 1894: 69. - Reference: Cleve (1894). - Remark: PÉTERFI (1993) reported this taxon from running waters.

*\#itzschia amphibia Grunow 1862: 574, pl. 28/12: fig. 23. - (Plate 85: Fig. 7) - References: Grunow (1862), HofMANN et al. (2013). - Remarks: PÉTERFI (1993) reported this taxon from mires. Rare in our recent study.

\#Nitzschia communis Rabenhorst 1860: no.949. - Reference: RABENHORST (1848-1860). - Remark: PÉTERFI (1993) reported this taxon from mires.

*\#itzschia frustulum (Kützing) Grunow in Cleve et Grunow 1880: 98. (Plate 85: Fig. 10) - References: Cleve and Grunow (1880), HofmanN et al. (2013). - Remarks: PÉTERFi (1993) reported this taxon from mires and lakes. In our recent study it was rarely found in Lake Capleror.

*\#Nitzschia hantzschiana Rabenhorst 1860: 40, pl. 6: fig. 6. - (Plate 85: Fig. 8) - References: Rabenhorst (1860), Hofmann et al. (2013). - Remarks: PÉTERFI (1993) reported this taxon from mires and running waters. In our recent study we found it in Lake Gales.

*\#Nitzschia palea var. debilis (Kützing) Grunow in Cleve et Grunow 1880: 96. - (Plate 85: Fig. 9) - References: CLEVE and GRunow (1880), HofmANn et 
al. (2013). - Remarks: PÉTERFI (1993) reported this taxon from mires and running waters. We found it in Lake Florica.

*\#itzschia perminuta - (see pp. 124-125, Plate 49).

*Nupela fennica (Hustedt) Lange-Bertalot in Krammer et Lange-Bertalot 2004: 440. - (Plate 85: Figs 11-12) - Reference: KRAMMER and LANGE-BERTALOT (2004). - Remark: This species was rarely found in Lake Lia in our recent study.

*Nupela impexiformis - (see pp. 126-127, Plate 50).

*Nupela lapidosa - (see pp. 128-129, Plate 51).

*Nupela paludigena - (see pp. 130-131, Plate 52).

*Nupela pocsii - (see pp. 132-133, Plate 53).

*Nupela silvabercynia (Lange-Bertalot) Lange-Bertalot in Lange-Bertalot et Metzeltin 1996: 97. - Reference: LANGe-Bertalot and Metzeltin (1996). - Remark: It was found only in the Lake Peleguta, rare.

*Nupela vitiosa - (see pp. 134-135, Plate 54).

*\#Orthoseira roeseana - (see pp. 136-137, Plate 55).

\#Placoneis elginensis (W. Gregory) E. J. Cox 1988: 155, figs 20-27, 3435, 45-46, 51. - References: Cox (1988), HofmANn et al. (2013). - Remark: PÉTERFI (1993) reported this taxon from running waters as Navicula elginensis.

*Planotbidium distinctum - (see pp. 138-139, Plate 56).

*Planothidium frequentissimum (Lange-Bertalot) Lange-Bertalot 1999: 282. - (Plate 85: Figs 20-23) - References: LANGe-Bertalot (1999a), HofmANN et al. (2013). - Remark: It was found in Lake Zanoaga in our recent study, rare.

*\#lanothidium lanceolatum - (see pp. 140-141, Plate 57).

*Planothidium oestrupii (A. Cleve) M. B. Edlund in M. B. Edlund et al. 2001: 88. - (Plate 85: Figs 15-17) - References: EdLund et al. (2001), HofmANN et al. (2013). - Remark: Only a few valves were found in Lake Gales.

*\#Platessa conspicua (Ant. Mayer) Lange-Bertalot in Krammer et LangeBertalot 2004: 445. - References: Krammer and LANge-Bertalot (2004), Hofmann et al. (2013). - Remarks: PÉterfi (1993) reported this taxon from running waters as Achnanthes conspicua. He also found $A$. pinnata in lakes that is probably conspecific with $P$. conspicuum. This name is currently regarded as a taxonomic synonym of $A$. conspicua. We found it in lake Lia.

*Psammotbidium altaicum - (see pp. 142-143, Plate 58).

*\#sammothidium helveticum - (see pp. 144-145, Plate 59).

*Psammothidium belveticum var. minor - (see pp. 146-147, Plate 60).

*\#sammotbidium kuelbsii - (see pp. 148-149, Plate 61).

*Psammothidium lauenburgianaum (Hustedt) L. N. Bukhtiyarova et Round 1996: 17, figs 62-65. - Reference: Bu KHTIYAROVA and Round (1996). - Remark: It was found only in Lake Stevia, rare: less than $1 \%$ in relative abundance.

*Psammothidium levanderi - (see pp. 150-151, Plate 62). 
*\#sammothidium marginulatum - (see pp. 152-153, Plate 63).

*Psammothidium microscopicum - (see pp. 154-155, Plate 64).

*Psammothidium montanum (Krasske) S. Mayama in S. Mayama, M. Idei, K. Osada et T. Nagumo 2002: 90. - (Plate 85: Figs 18-19) - Reference: MAYAMA et al. (2002). - Remark: This species was found in Lake Pietrelice, rare, less than $1 \%$ in relative abundance.

*\#sammothidium rossii - (see pp. 156-157, Plate 65).

*Psammothidium scoticum - (see pp. 158-159, Plate 66).

*\#sammothidium subatomoides - (see pp. 160-161, Plate 67).

*Pseudostaurosira parasitica (W. Smith) Morales 2003: 287. - Reference: MoRALES and EDLUND (2003). - Remark: Only one valve was found in the Lake Stirbu.

*Pseudostaurosira parasitica var. subconstricta (Grunow) E. Morales in E. Morales et Edlund 2003: 287. - Reference: Morales and EdLund (2003). Remark: A few valves were found in the Lake Stirbu.

*Pseudostaurosira pseudoconstruens - (see pp. 162-163, Plate 68).

*Pseudostaurosiropsis E. A. Morales 2001: 117, fig. 7a-1. - (Plate 85: Figs 24-27) - Reference: Morales (2001). - Remark: Probably the representatives of this genus were found only in Lake Peleguta.

*\#Reimeria sinuata (W. Gregory) Kociolek et Stoermer 1987: 457, figs 1-10. - References: Kociolek and Stoermer (1987), HofmAnn et al. (2013). - Remarks: PÉTERfi (1993) reported it from running waters as Cymbella sinuata. Rare in our recent study, only a few valves were found in Lake Lia.

*\#Rossitbidium nodosum (Cleve) Aboal in Aboal, Alvarez Cobelas, Cambra et Ector 2003: 178. - Reference: ABOAL et al. (2003). - Remarks: PÉTERfi (1993) reported it from mires and glacial lakes, as Achnanthes nodosa. Rare in our recent study, in the Lake Brazi.

*\#Sellaphora bacillum (Ehrenberg) Mann 1989: 2, figs 2, 9, 13-14, 18, 39-40. - References: MANN (1989), Hofmann et al. (2013). - Remarks: PÉterfi (1993) found it in lakes, and published as Navicula bacillum. Rare in our recent study.

*Sellaphora elorantana - (see pp. 164-165, Plate 69).

* Sellaphora hentiensis (Kulikovskiy, Lange-Bertalot, A. Witkowski et N. I. Dorofeyuk) C. E. Wetzel et L. Ector in Wetzel, Ector, Van de Vijver, Compère et Mann 2015: 226. - References: Kulikovskiy et al. (2010), Wetzel et al. (2015). - Remark: We found it in Lake Gemenele, Peleguta and Stirbu.

*Sellaphora laticeps (Hustedt) C. E. Wetzel, L. Ector, B. Van de Vijver, Compère et D. G. Mann 2015: 226. - Reference: Wetzel et al. (2015). - Remark: This species was found in Lake Florica, Lezilor, Pietrele, Stirbu and Viorica.

\#Sellaphora medioconvexa (Hustedt) Wetzel in Wetzel, Ector, Van de Vijver, Compère et Mann 2015: 227. - Reference: Wetzel et al. (2015). - Remark: PÉTERFI (1993) reported it from glacial lakes as Navicula medioconvexa. 
*Sellaphora nigri - (see pp. 166-167, Plate 70).

*\#Sellaphora pupula (Kützing) Mereschkowsky 1902: 187, pl. 4: figs 1-5. - References: Meresch kowsky (1902), Hofmann et al. (2013). - Remarks: PÉTERFI (1993) reported it from running waters as Navicula pupula. In our recent study, it was found in the Lake Ana, Peleguta, Pietrele, Stirbu and Zanoaga. Not rare.

*Sellaphora radiosa (Hustedt) H. Kobayasi in S. Mayama, M. Idei, K. Osada et T. Nagumo 2002: 90. - Reference: Mayama et al. (2002). - Remark: In our recent study not rare, it was found in the Lake Ana, Gales and Viorica.

*\#Sellaphora rectangularis (W. Gregory) Lange-Bertalot et Metzeltin 1996: 102, pl. 25: figs 10-12, pl. 125: fig. 7. - References: LANGE-BERTALOT and METZELTiN (1996), HofmAnN et al. (2013). - Remarks: PÉTERfi (1993) reported it from mires and running waters as Navicula pupula var. rectangularis. Rare in the Lake Lia.

*Sellaphora stauroneioides - (see pp. 168-169, Plate 71).

* Sellaphora tridentula (Krasske) Wetzel in Wetzel, Ector, Van de Vijver, Compère et Mann 2015: 227. - (Plate 85: Fig. 6) - Reference: Wetzel et al. (2015). Remarks: In our recent study only one valve was found in Lake Viorica. Very rare.

*Stauroforma exiguiformis - (see pp. 170-171, Plate 72).

*Stauroneis acidoclinata - (see pp. 172-173, Plate 73).

*\#Stauroneis anceps Ehrenberg 1843: 306, 422, pl. 2/1: fig. 18. - Reference: Ehrenberg (1843). - Remarks: PÉterfi (1993) reported it from mires, lakes and glacial lakes. It is a common, but not abundant species. In our recent study we found it in all studied lake, its constancy is 5 (100\%).

*Stauroneis neofossilis - (see pp. 174-175, Plate 74).

\#Stauroneis obtusa Lagerstedt 1873: 36, pl. 1: fig. 11. - Reference: LAGERSTEDT (1873). - Remarks: PÉTERFi (1993) found this species in mires. He also reported Stauroneis lapponica from mires. S. lapponica is currently regarded as a taxonomic synonym of Stauroneis obtusa Lagerstedt.

* Stauroneis phoenicenteron - (see pp. 176-177, Plate 75).

\#Stauroneis smithii Grunow 1860: 564, pl.4: fig. 16. - References: GRUNOW (1860), Hofmann et al. (2013). - Remark: PÉTERFi (1993) reported this species from running waters of Retezat Mountains.

\#Stauroneis thermicola (Petersen) Lund 1946: 61, fig. 3. - References: Lund (1946), HofmANn et al. (2013). - Remark: PÉTERFi (1993) reported this species from running waters of Retezat Mountains.

*\#Staurosira construens Ehrenberg 1843: 424. - References: EHRENBERG (1843), Hofmann et al. (2013) as Fragilaria construens. - Remarks: PÉterfi (1993) reported this species from mires as Fragilaria construens. In our recent study it was rare in the Lake Peleaga.

* \#taurosira construens var. binodis (Ehrenberg) Hamilton in Hamilton, Poulin, Charles et Angell 1992: 29. - Reference: Hamilton et al. (1992). - 
Remarks: PÉTERfi (1993) reported this species from glacial lakes and running waters as Fragilaria construens $\mathrm{f}$. binodis. In our recent study it was rare, we found it only in the Lake Lia.

*Staurosira parasitoides Lange-Bertalot, Schmidt et Klee in Schmidt, Lange-Bertalot et Klee 2004: 3, figs 1-5. - Reference: ScHmid et al. (2004). Remark: Very rare in our recent study, only one valve was found in Lake Peleguta.

*\#Staurosira venter - (see pp. 178-179, Plate 76).

\#Staurosirella leptostauron (Ehrenberg) D. M. Williams et Round 1988: 276, figs 22-23. - Reference: Williams and Round (1988a, '1987'). - Remark: PÉTERFI (1993) reported this species from running waters as Fragilaria leptostauron.

*\#Staurosirella pinnata - (see pp. 180-181, Plate 77).

*Staurosirella sp. - (see pp. 182-183, Plate 78).

*Stenopterobia delicatissima - (see pp. 184-185, Plate 79).

\#Stephanodiscus astraea (Kützing) Grunow 1880: 114. - Reference: GRUNOW (1880). - Remark: PÉTERFI (1993) reported this species from mires, lakes and also from running waters.

*Surirella angusta - (see pp. 186-187, Plate 80).

*\#Surirella bifrons - (see pp. 186-187, Plate 80).

* Surirella linearis - (see pp. 188-191, Plates 81-82).

* Surirella linearis var. constricta Grunow 1862: 455. - Reference: GRUNOW (1862). - Remark: Only one valve was found in Lake Stirbu in our recent study.

*Surirella tenera - (see pp. 186-187, Plate 80).

\#Surirella spiralis Kützing 1844: 60, pl. 3: fig. 64. - Reference: KüTZING (1844). - Remark: PÉTERFI (1993) reported it from running waters.

*\#abellaria fenestrata (Lyngbye) Kützing 1844: 127, pl. 17: fig. 22; pl. 18: fig. 2. - (Plate 85: Fig. 30) - References: KütZING (1844), HofMANN et al. (2013). - Remarks: PÉTERFI (1993) reported it from mires and glacial lakes. We found T. fenestrata in the Lake Brazi, Bucura, Pietrelice-2, Pietrelice-3 and Slavieu in our recent study. Never abundant.

*\#Tabellaria flocculosa - (see pp. 192-193, Plate 83).

*Tetracyclus rupestris (Kützing) Grunow in Van Heurck 1881: pl. LII [52]: figs 13-14. - (Plate 85: Figs 28-29) - References: VAN HeurCK (1881), HofmANn et al. (2013). - Remarks: We found it only in the Lake Lia. Rare.

\#Ulnaria ulna var. aequalis (Kützing) Aboal in Aboal, Alvarez Cobelas, Cambra et Ector 2003: 112. - Reference: ABOAL et al. (2003). - Remark: PÉTERFI (1993) reported it from running waters.

\#Ulnaria ulna var. amphirhynchus (Ehrenberg) Aboal in Aboal, Alvarez Cobelas, Cambra et Ector 2003: 113. - Reference: ABOAL el al. (2003). - Remark: PÉTERFI (1993) reported it from running waters as Synedra ulna var. amphirbynchus. 
Achnanthes oblongella Østrup 1902: 252 (34), pl. I: fig. 9

(Plate 1: Figs 1-17)

References: Østrup (1902), Krammer and LANGe-Bertalot (1991), Potapova (without year).

Distribution in glacial lakes in the Retezat Mountains

\begin{tabular}{ll}
\hline Lakes & Florica, Gales, Gemenele, Lezilor, Lia, Negru, Peleguta, Pietrele, \\
& Pietrelice-1, Pietrelice-2, Pietrelice-3, Stanisoara, Stevia, Stirbu, \\
& Turcelu, Viorica, Zanoaga \\
Relative abundance (max.) & $5.23 \%$ \\
Constancy & $4(74 \%)$ \\
\hline
\end{tabular}

Remarks: PÉTERFi (1993) published it from mires and glacial lakes probably as Achnanthes saxonica. This taxon is one of the most common diatom in the Retezat Mts. 


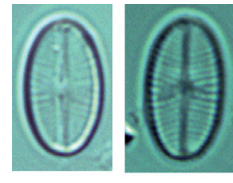

1

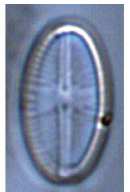

3

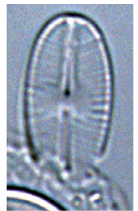

4
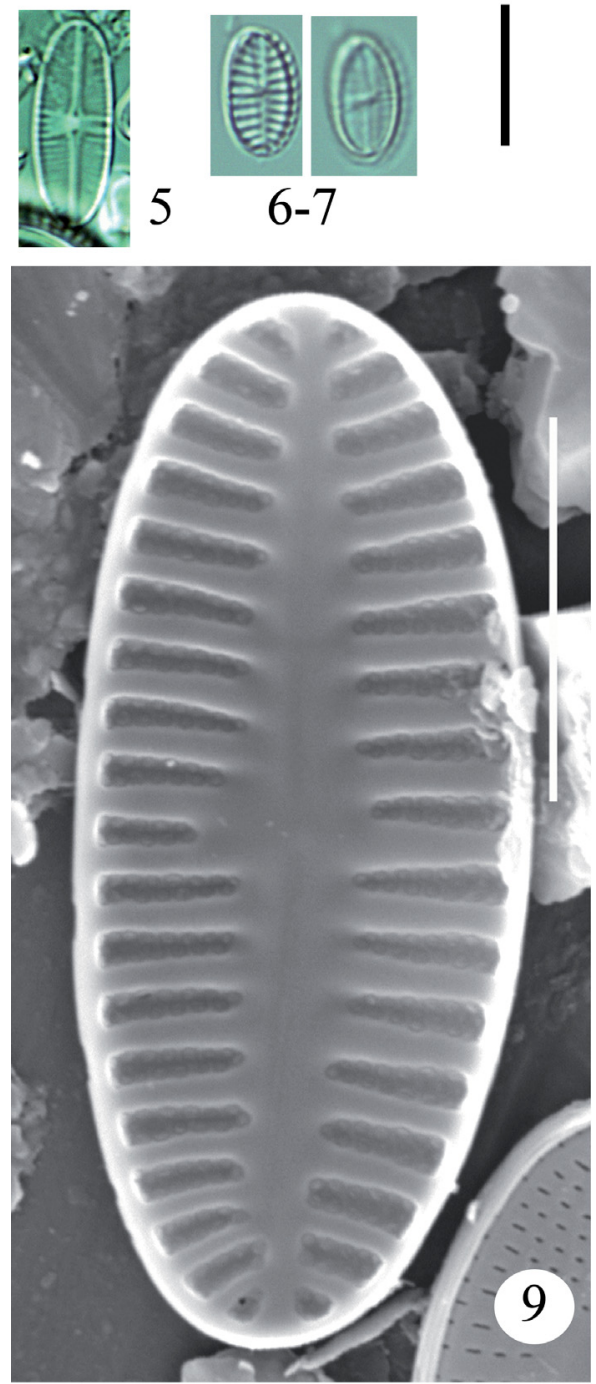
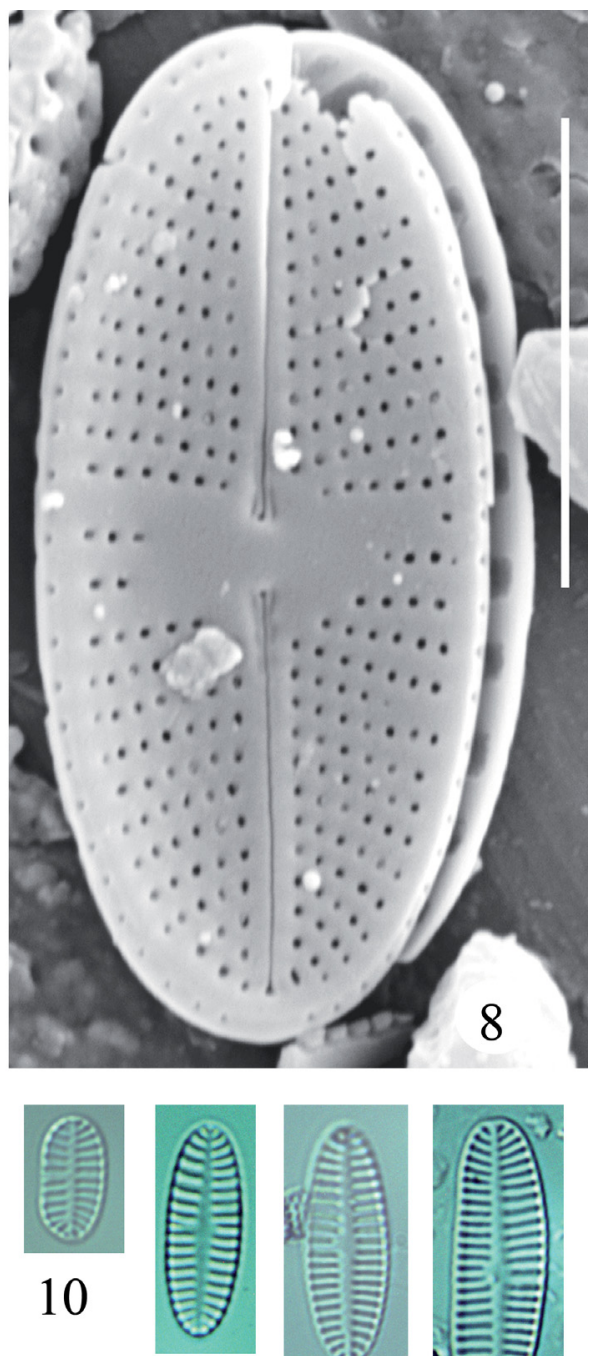

11

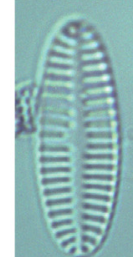

12

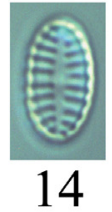

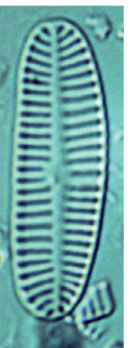

13

Plate 1: Achnanthes oblongella. - Figs 1-17: Lake Gales. Figs 1-5: Raphe valve, LM. Figs 6-7: Rapheless and raphe valves, pictures of the same specimens at different focal planes, LM. Fig. 8: Raphe valve, outside view, SEM. Fig. 9: Rapheless valve, inside view, SEM. Figs 10-14: Rapheless valve, LM. Figs 15-17: The same frustule at different focal planes. Scale bars $=10 \mu \mathrm{m}$. 


\section{Achnanthidium minutissimum (Kützing) Czarnecki 1994: 157} (Plate 2: Figs 1-15)

References: KRAMMER and LANGe-Bertalot (1991), CZARneCKi (1994), Potapova (2009a), HofmAnn et al. (2013).

Distribution in glacial lakes in the Retezat Mountains

\begin{tabular}{ll}
\hline Lakes & Ana, Bucura, Caprelor, Gales, Gemenele, Lezilor, Lia, Negru, Pe- \\
& leaga, Pietrele, Pietrelice-1, Slavieu, Stirbu, Viorica, Zanoaga \\
Relative abundance (max.) & $3.6 \%$ \\
Constancy & $4(65 \%)$ \\
\hline
\end{tabular}

Remarks: PÉTERfi (1993) reported this taxon from running water as Achnanthes affinis. Achnanthidium minutissimum is one of the most common diatoms. 

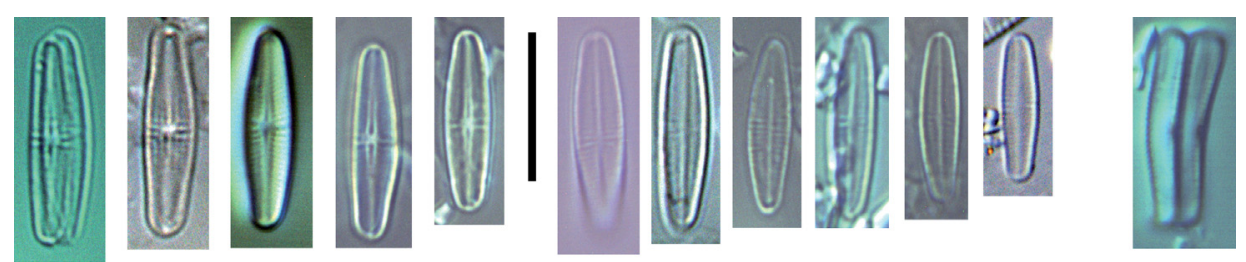

$1-5$

$6-11$

12

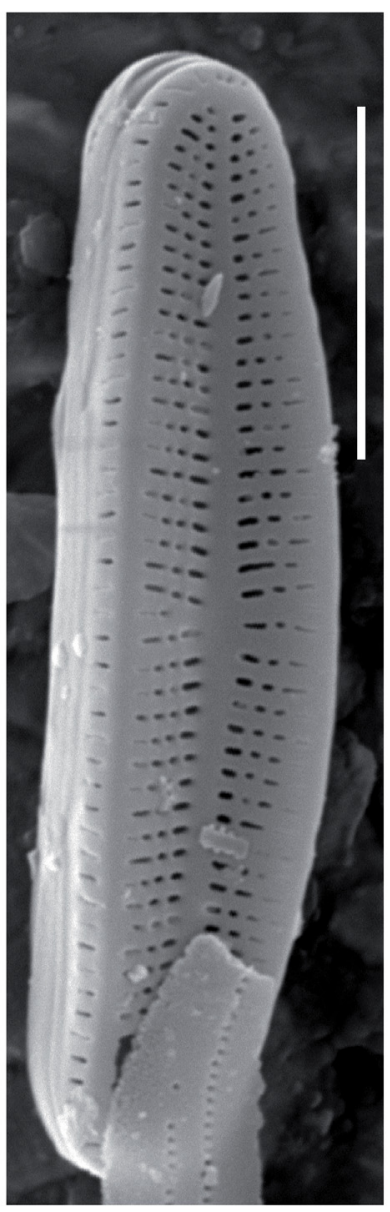

13

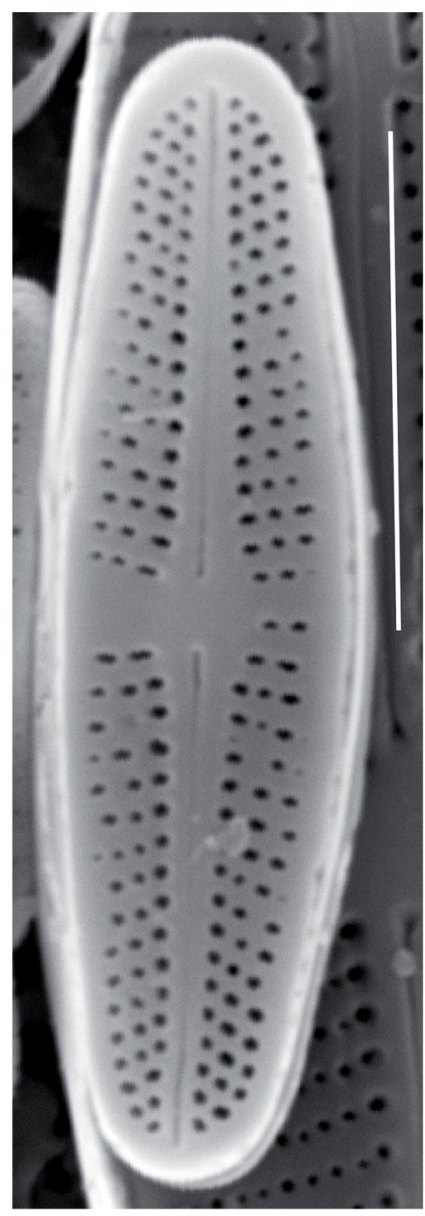

14

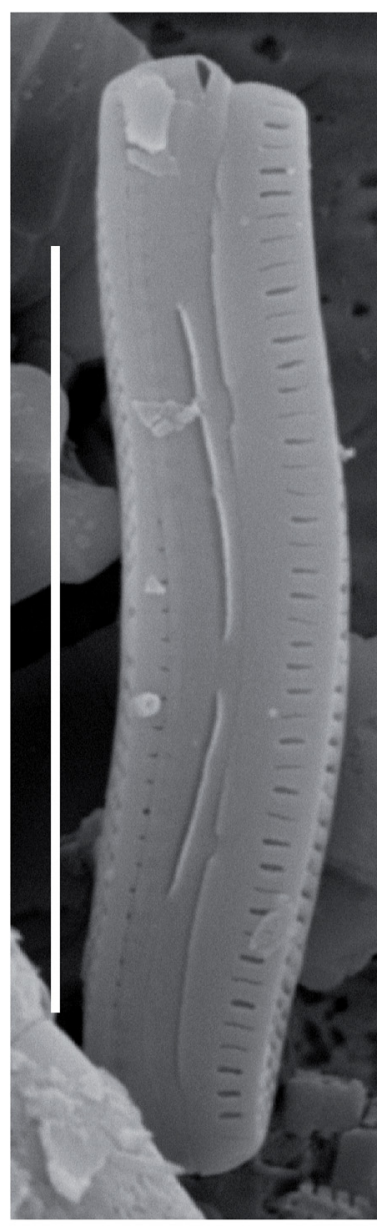

15

Plate 2: Achnanthidium minutissimum. - Figs 1-5: Lake Lia, raphe valves, LM. Figs 6-11: Lake Lia, rapheless valves, LM. Fig. 12: Lake Lia, girdle view, LM. Fig. 13: Lake Lia, rapheless valve, outside view, SEM. Fig. 14: Lake Lia, raphe valva, outside view, SEM. Fig. 15: Lake Lia, girdle view, SEM. Scale bars $=10 \mu \mathrm{m}$. 
Amphora copulata (Kützing) Schoeman et E. M. Archibald 1986: 429 , figs $11-13,30-34$

(Plate 3: Figs 1-8)

References: Schoeman and ARChibald (1986), Krammer and LANGeBertalot (1999a), Levkov (2009), Stepanek and Kociolek (2011), HofMANN et al. (2013).

Distribution in glacial lakes in the Retezat Mountains

\begin{tabular}{ll}
\hline Lakes & Lia, Peleaga, Peleguta \\
Relative abundance (max.) & $1.5 \%$ \\
Constancy & $1(12 \%)$ \\
\hline
\end{tabular}

Remarks: PÉterfi (1993) found this species in lakes and running water. It reported as Amphora ovalis var. pediculus; and Amphora perpusilla. Rare in our recent study. 

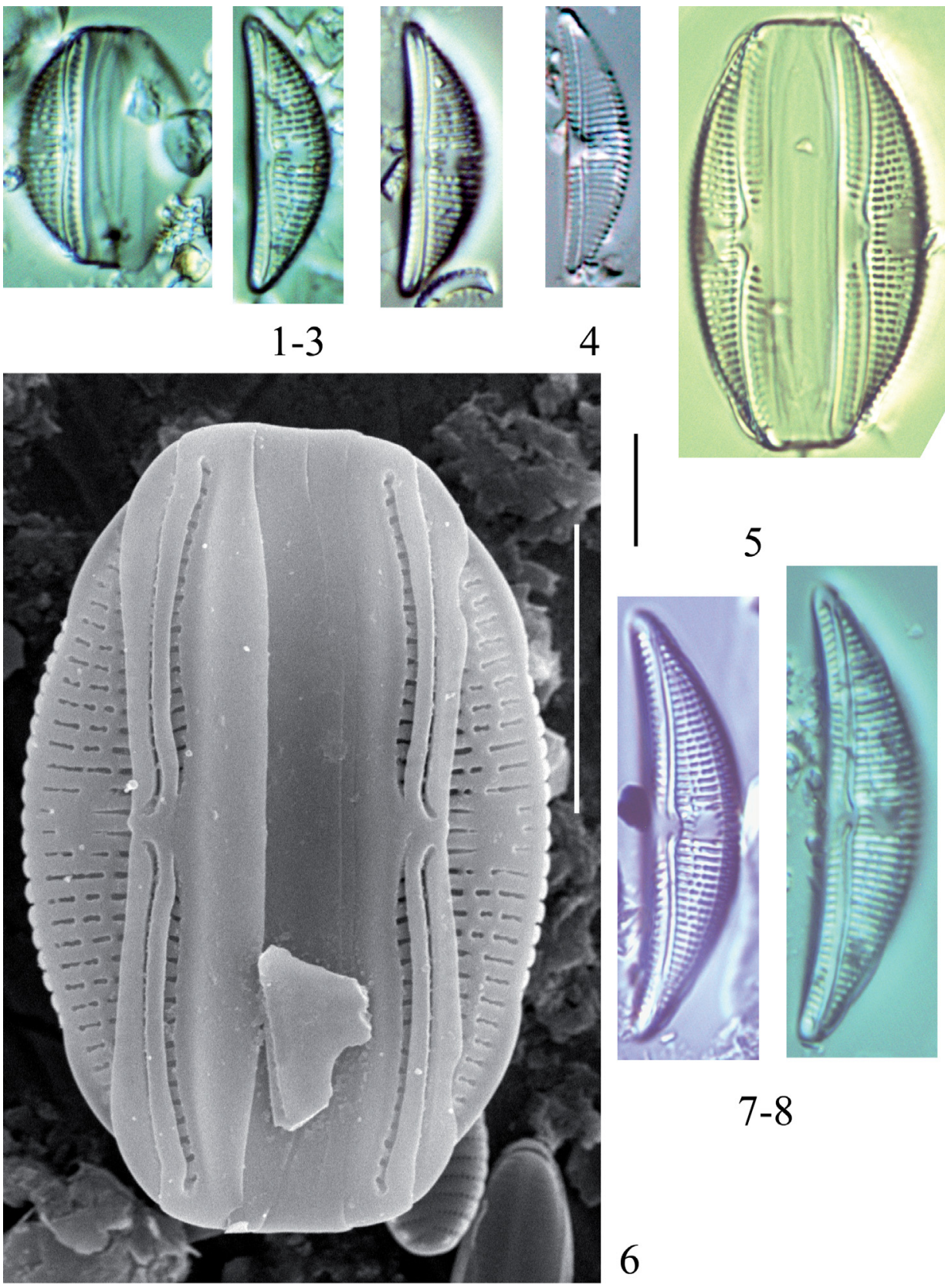

Plate 3: Amphora copulata. - Figs 1-3: Lake Peleguta, LM. Figs 4-5: Lake Gales, LM. Fig. 6: Lake Peleguta, inside view, SEM. Figs 7-8: Lake Peleguta, LM. Scale bars $=10 \mu \mathrm{m}$. 
Amphora inariensis Krammer 1980: 211, pl. 4: figs 21-24, pl. 6: figs

$$
\text { 36-37, 43-45 }
$$

(Plate 4: Figs 1-11)

References: KRAMMER (1980), LeVKov (2009).

Distribution in glacial lakes in the Retezat Mountains

\begin{tabular}{ll}
\hline Lakes & Gales \\
Relative abundance (max.) & $1 \%$ \\
Constancy & $1(4 \%)$ \\
\hline
\end{tabular}

Remark: Very rare species. 

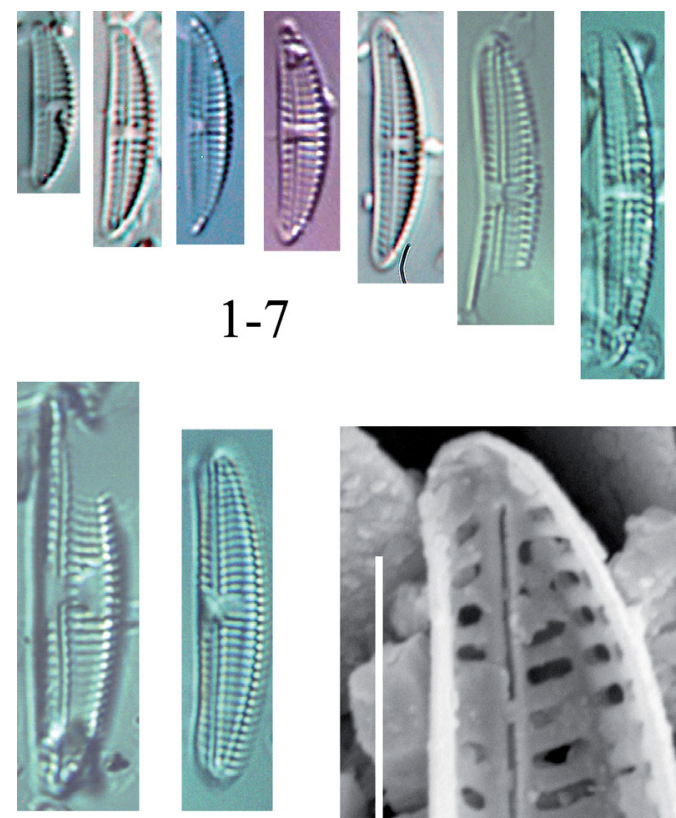

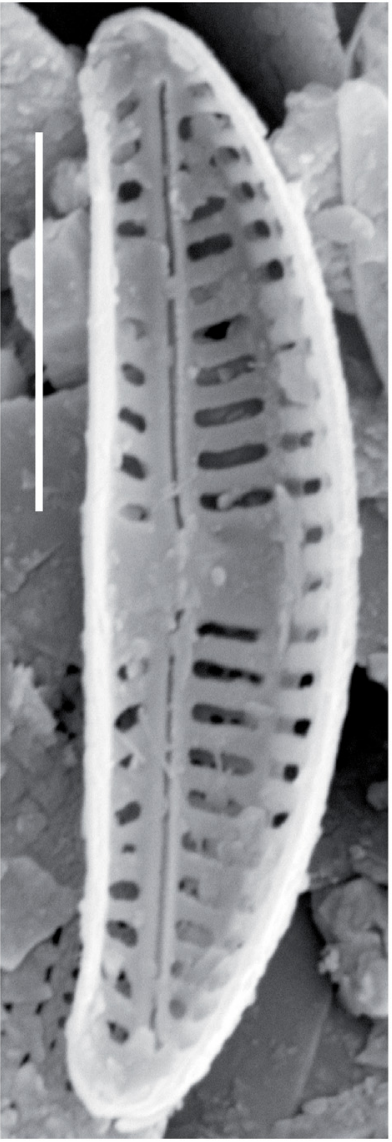

10

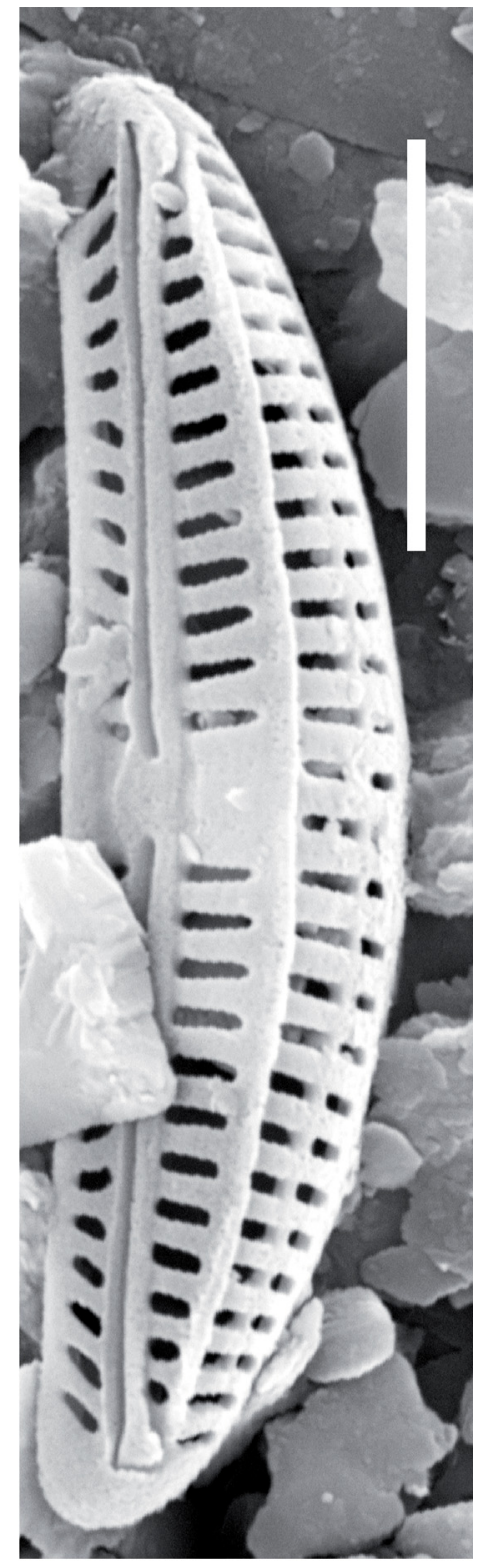

11

Plate 4: Amphora inariensis. - Figs 1-7: Lake Lia, LM. Figs 8-9: Lake Gales, LM. Figs 10-1 1: Lake Lia, outside view, SEM. Scale bars $=10 \mu \mathrm{m}$. 


\section{Aulacoseira alpigena (Grunow) Krammer 1991: 93, figs 1-15} (Plate 5: Figs 1-10)

References: Krammer (1991), Houk (2003), Kawecka and Galas (2003), Potapova (2009b), Buczkó et al. (2013a).

Distribution in glacial lakes in the Retezat Mountains

\begin{tabular}{ll}
\hline Lakes & Ana, Brazi, Bucura, Caprelor, Florica, Gales, Gemenele, Lezilor, \\
& Lia, Negru, Peleaga, Peleguta, Pietrele, Pietrelice-1, Pietrelice-2, \\
& Pietrelice-3, Slavieu, Stanisoara, Stevia, Stirbu, Turcelu, Viorica, \\
& Zanoaga \\
Relative abundance (max.) & $72 \%$ \\
Constancy & $5(100 \%)$ \\
\hline
\end{tabular}

Remarks: PÉTERFI (1993) reported this taxon from lakes and running waters as Melosira distans var. alpigena. Aulacoseira alpigena is very abundant and common in the Retezat Mts. Abundant, common species in our recent study. 

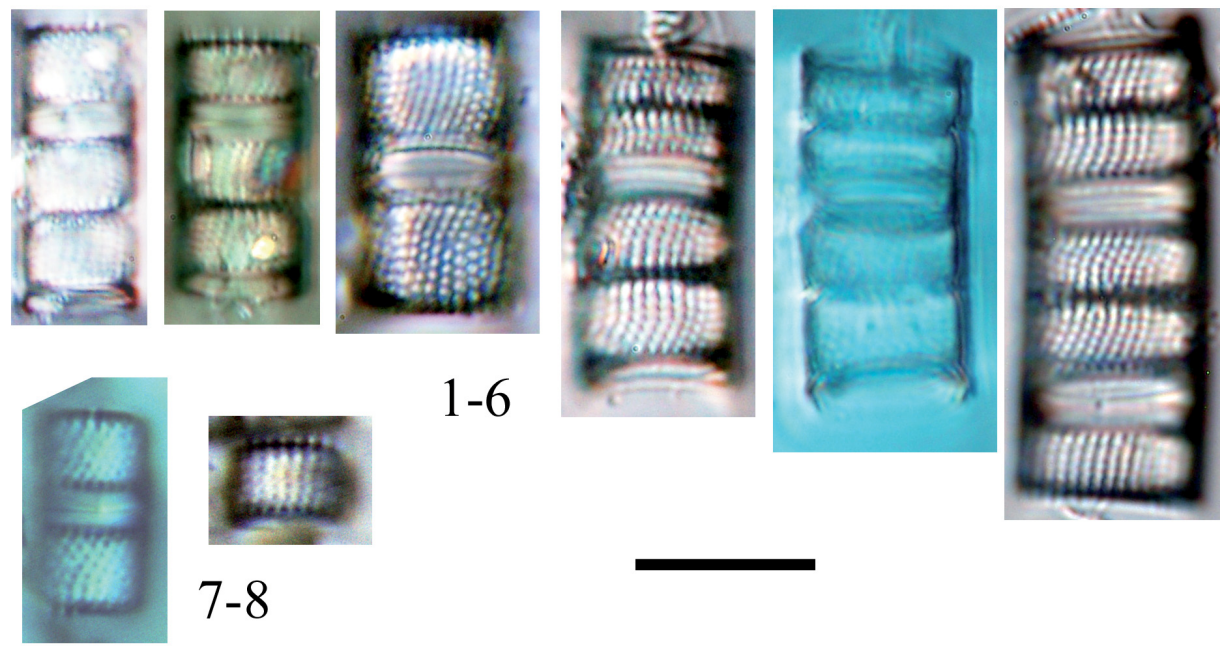

$1-6$

\section{$7-8$}

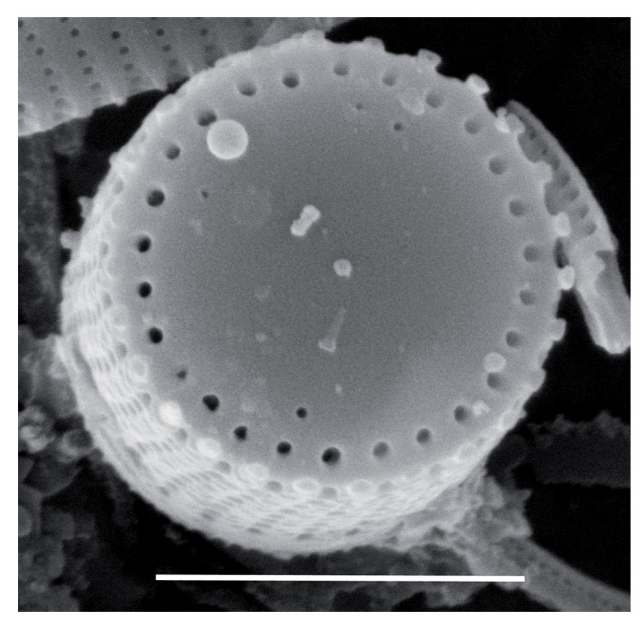

9

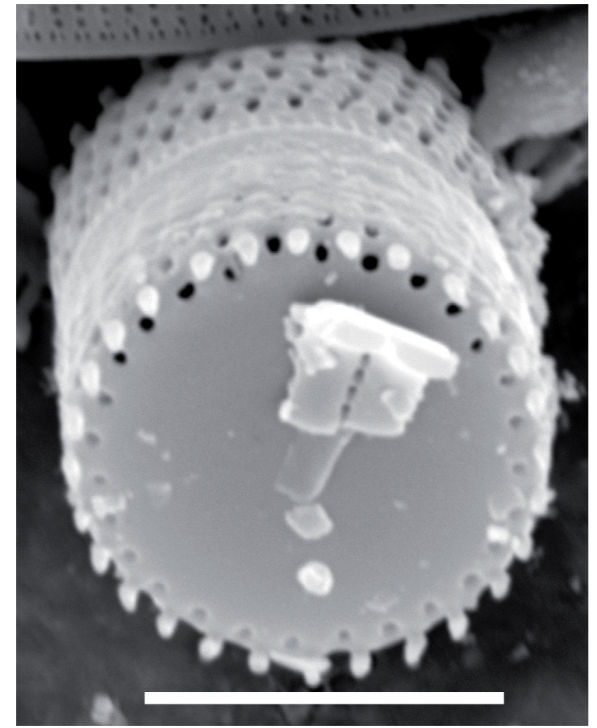

10

Plate 5: Aulacoseira alpigena. - Figs 1-6: Lake Brazi, LM. Figs 7-8: Lake Ana, LM. Figs 9-10: Lake Brazi, SEM. Scale bars $=10 \mu \mathrm{m}$ (Figs 1-8), $5 \mu \mathrm{m}$ (Figs 9-10). 


\section{Aulacoseira ambigua (Grunow) Simonsen 1979: 56 \\ (Plate 6: Figs 1-10)} (2010).

References: Simonsen (1979), Houk (2003), Potapova and English Distribution in glacial lakes in the Retezat Mountains

\begin{tabular}{ll}
\hline Lakes & $\begin{array}{l}\text { Ana, Brazi, Bucura, Caprelor, Gales, Lezilor, Lia, Negru, Peleaga, } \\
\text { Peleguta, Pietrele, Pietrelice-3, Slavieu, Stanisoara, Turcelu, Vio- } \\
\text { rica, Zanoaga }\end{array}$ \\
Relative abundance (max.) & $0.5 \%$ \\
Constancy & $1(4 \%)$ \\
\hline
\end{tabular}



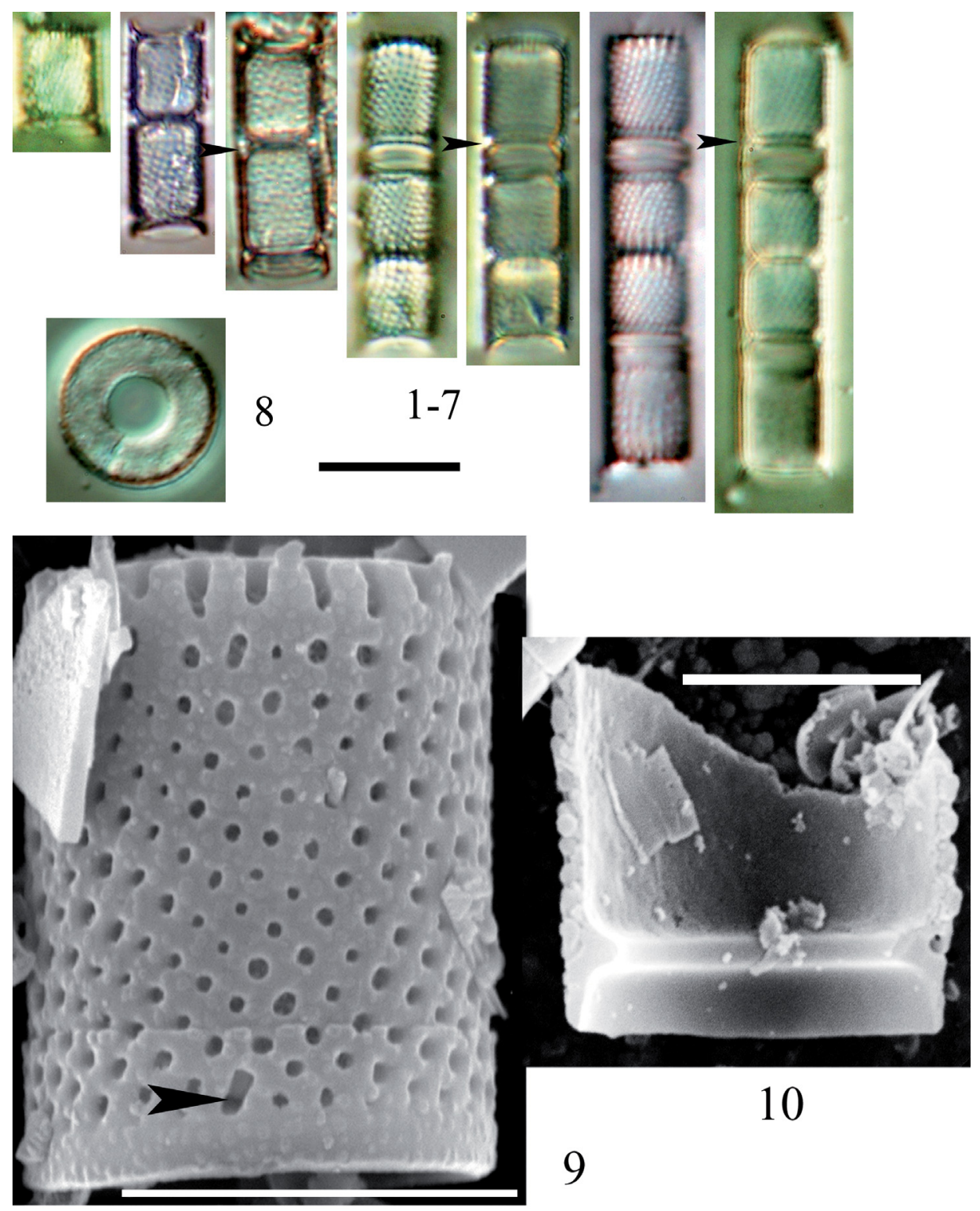

Plate 6: Aulacoseira ambigua. - Figs 1-8: Lake Brazi, LM. Figs 9-10: Lake Brazi, SEM. Figs 4-5 and 6-7 are pictures of the same specimens at different focal planes. Figs 8, 10: Ringleiste. Fig. 9: Mantle view. Arrow indicates the external opening of the rimoportula. Scale bar $=10 \mu \mathrm{m}$ (Figs 1-8), $5 \mu \mathrm{m}$ (Figs 9-10). 
Aulacoseira laevissima (Grunow) Krammer 1991: 98

(Plate 7: Figs 1-7)

References: KRAmmer (1991), Houk (2003).

Distribution in glacial lakes in the Retezat Mountains

\begin{tabular}{ll}
\hline Lakes & Brazi, Stavieau \\
Relative abundance $(\max )$. & $0.5 \%$ \\
Constancy & $1(8 \%)$ \\
\hline
\end{tabular}

Remark: Very rare species in our recent study. 


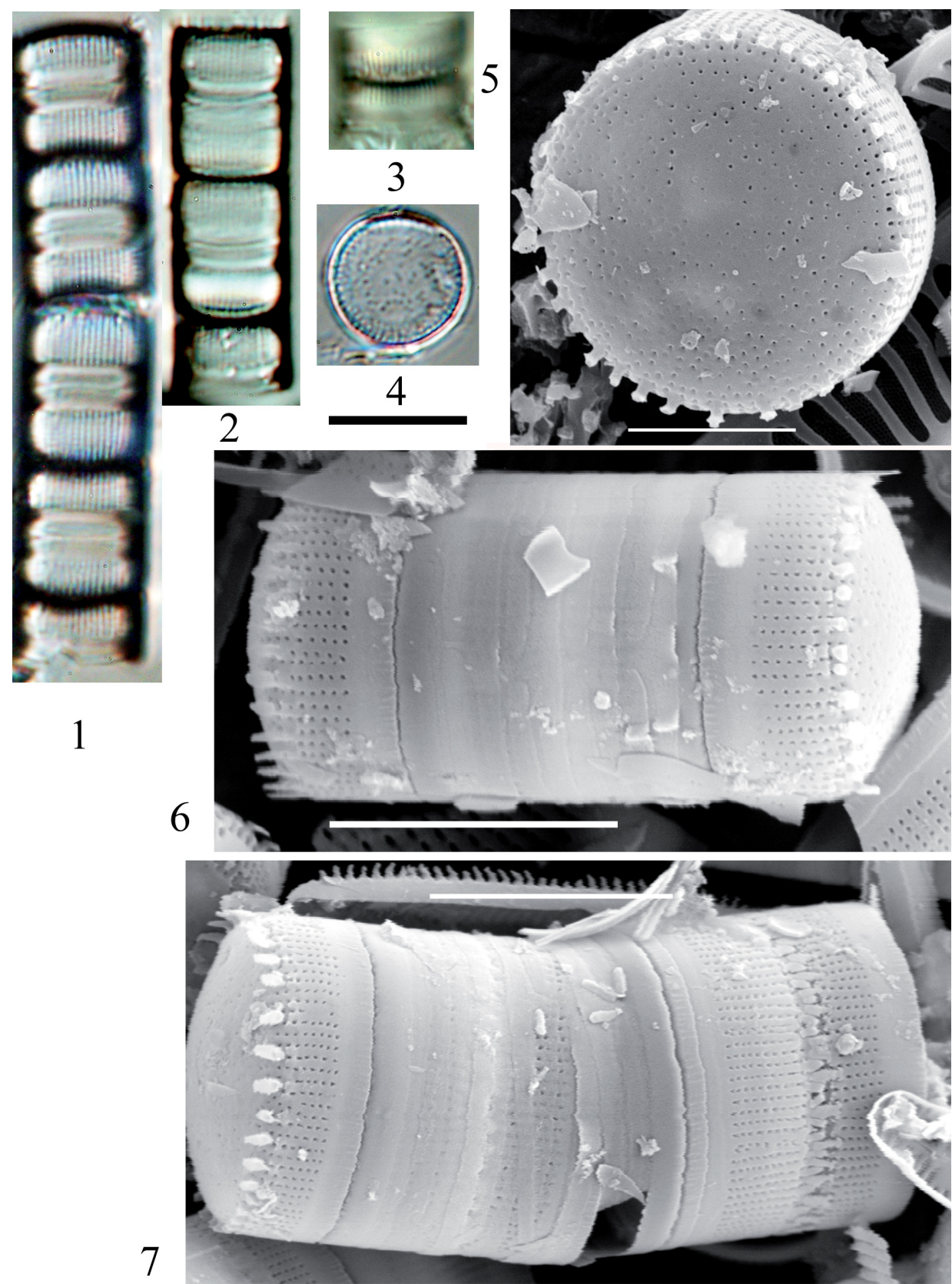

Plate 7: Aulacoseira laevissima. - Figs 1-3: Lake Brazi, mantle view, LM. Fig. 4: Lake Brazi, valve view, LM. Fig. 5: Lake Brazi, valve view, SEM. Figs 6-7: Lake Brazi, SEM. Scale bar $=10 \mu \mathrm{m}$ (Figs $1-5), 5 \mu \mathrm{m}$ (Figs $5-7$ ). 
Aulacoseira nivalis (W. Smith) English et Potapova 2009: 39 (Plate 8: Figs 1-14)

References: Houk (2003), English and Potapova (2009), Potapova and ENGLISH (2011).

Distribution in glacial lakes in the Retezat Mountains

\begin{tabular}{ll}
\hline Lakes & Bucura, Florica, Gemenele, Lezilor, Lia, Negru, Pietrelice-1, \\
& Pietrelice-2, Pietrelice-3, Slavieu, Stanisoara, Turcelu, Viorica, \\
& Zanoaga \\
Relative abundance $(\max )$. & $3 \%$ \\
Constancy & $4(61 \%)$ \\
\hline
\end{tabular}

Remark: Probably it is conspecific with Melosira distans var. distans and known from mires and lakes (PÉTERFI 1993). 


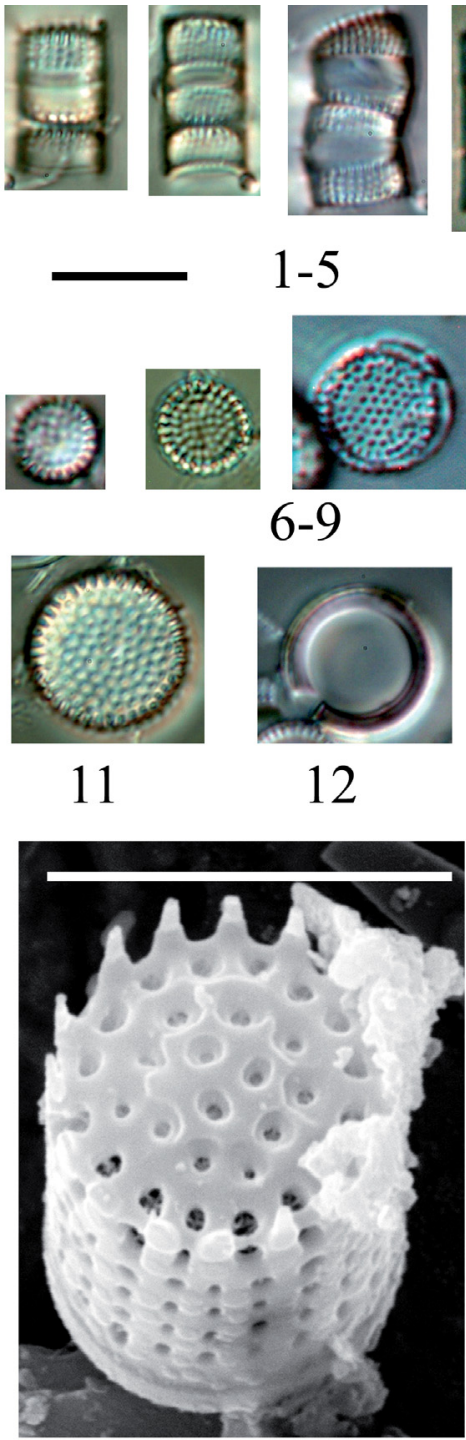

13
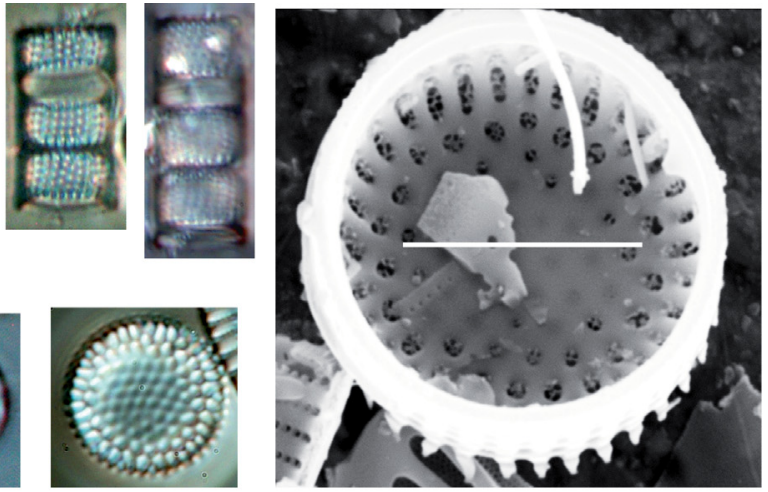

10

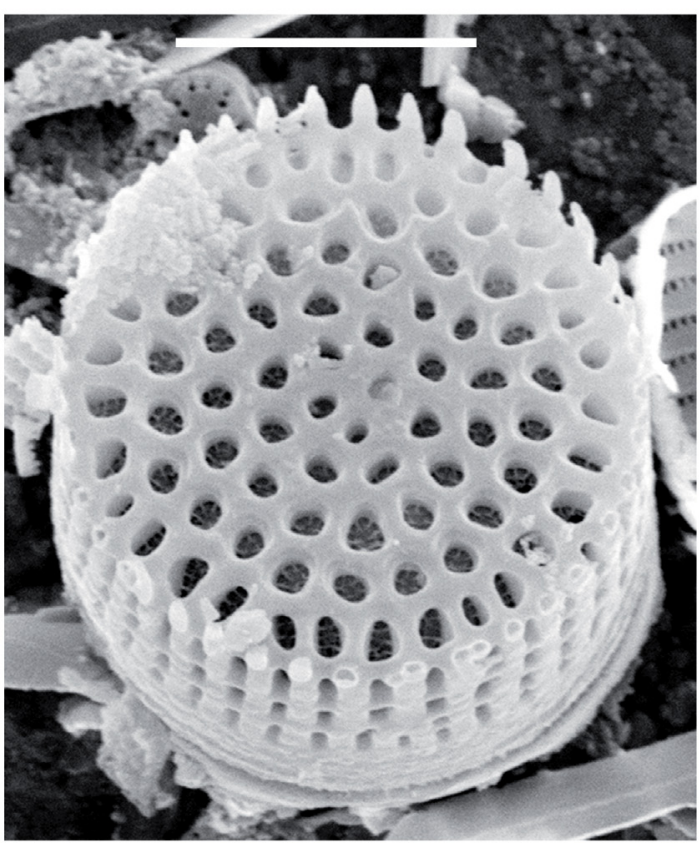

14

Plate 8: Aulacoseira nivalis. - Figs 1-5: Lake Brazi, mantle view, LM. Figs 6-9, 11-12: Lake Brazi, valve view, LM. Fig. 10: Lake Brazi, inside view, SEM. Figs 13-14: Lake Brazi, outside view, SEM. Scale bar $=10 \mu \mathrm{m}$ (Figs 1-9, 11-12), $5 \mu \mathrm{m}$ (Figs 10, 13-14). 
Aulacoseira perglabra (Østrup) E. Y. Haworth 1990: 195

(Plate 9: Figs 1-15)

References: HAWORTH (1990), HOUK (2003).

Distribution in glacial lakes in the Retezat Mountains

Lakes

Ana, Bucura, Florica, Gales, Negru, Pietrele, Pietrelice-1, Pietre-

lice-2, Pietrelice-3, Slavieu, Stanisoara

Relative abundance ( $\max ) \quad 5 \$.

Constancy $3(48 \%)$

Remark: Common but not abundant species in our recent study. 

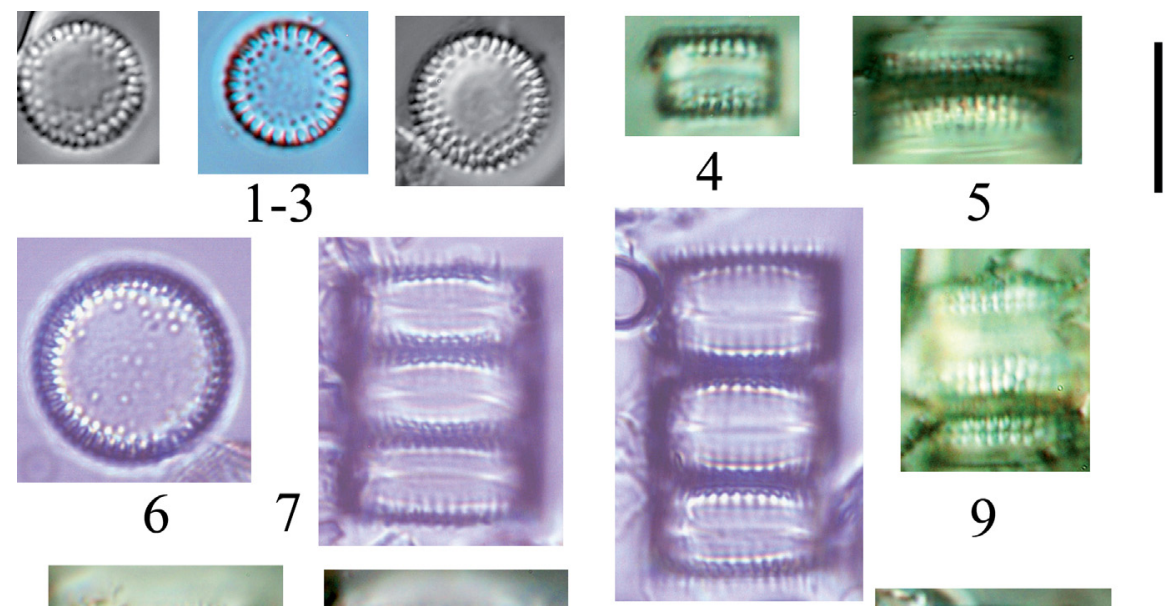

5

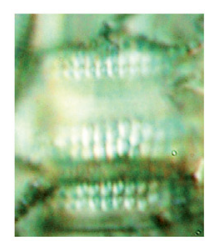

9
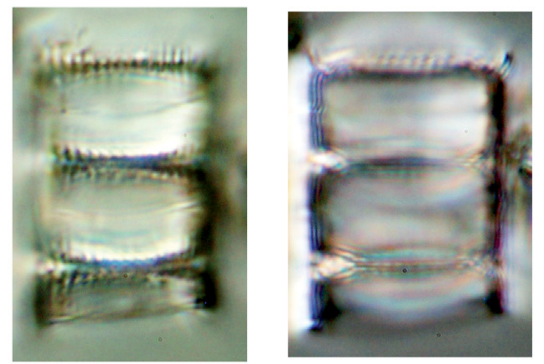

10-11

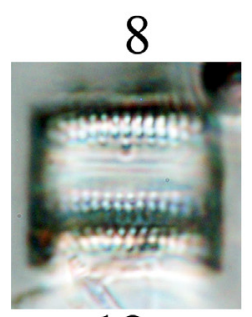

12

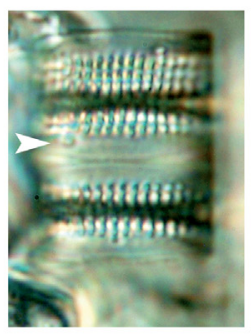

13

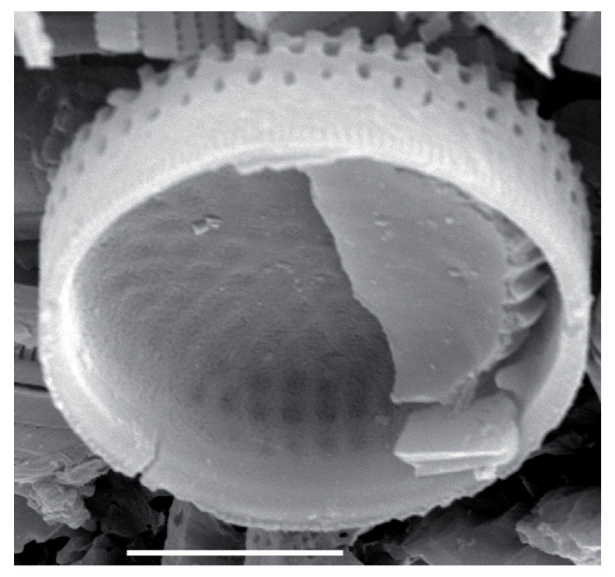

14

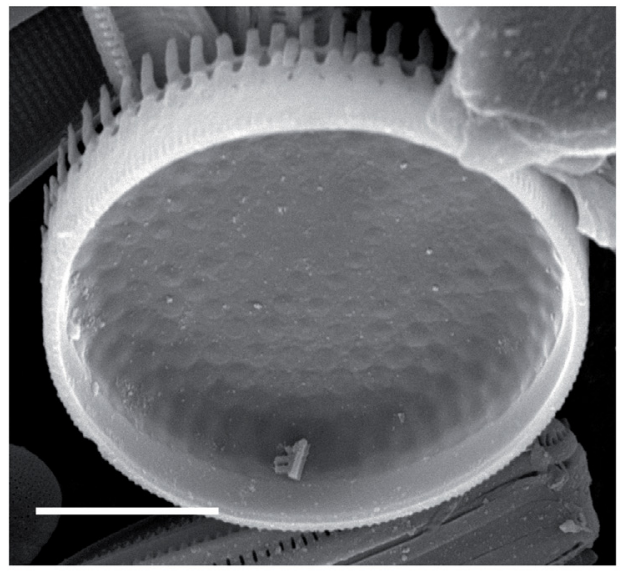

15

Plate 9: Aulacoseira perglabra. - Figs 1-3: Lake Brazi, valve view, LM. Figs 4-5: Lake Brazi, mantle view, LM. Fig. 6: Lake Negru, valve view, LM. Figs 7-8: Lake Negru, mantle view, LM. Figs 9-13: Lake Brazi, mantle view, LM. Figs 10-11 are pictures of the same specimens at different focal planes. Figs 14-15: Lake Brazi, mantle, inside view, SEM. Scale bar $=10 \mu \mathrm{m}$ (Figs 1-13), 5 $\mu \mathrm{m}$ (Figs 14-15). 


\section{Aulacoseira pfaffiana (Reinsch) Krammer 1991: 94, figs 45-54 (Plate 10: Figs 1-7)}

References: KRAMMER (1991), HOUK (2003).

\section{Distribution in glacial lakes in the Retezat Mountains}

Relative abundance (max.) $\quad 3 \%$

Remark: Common species in our recent study. 

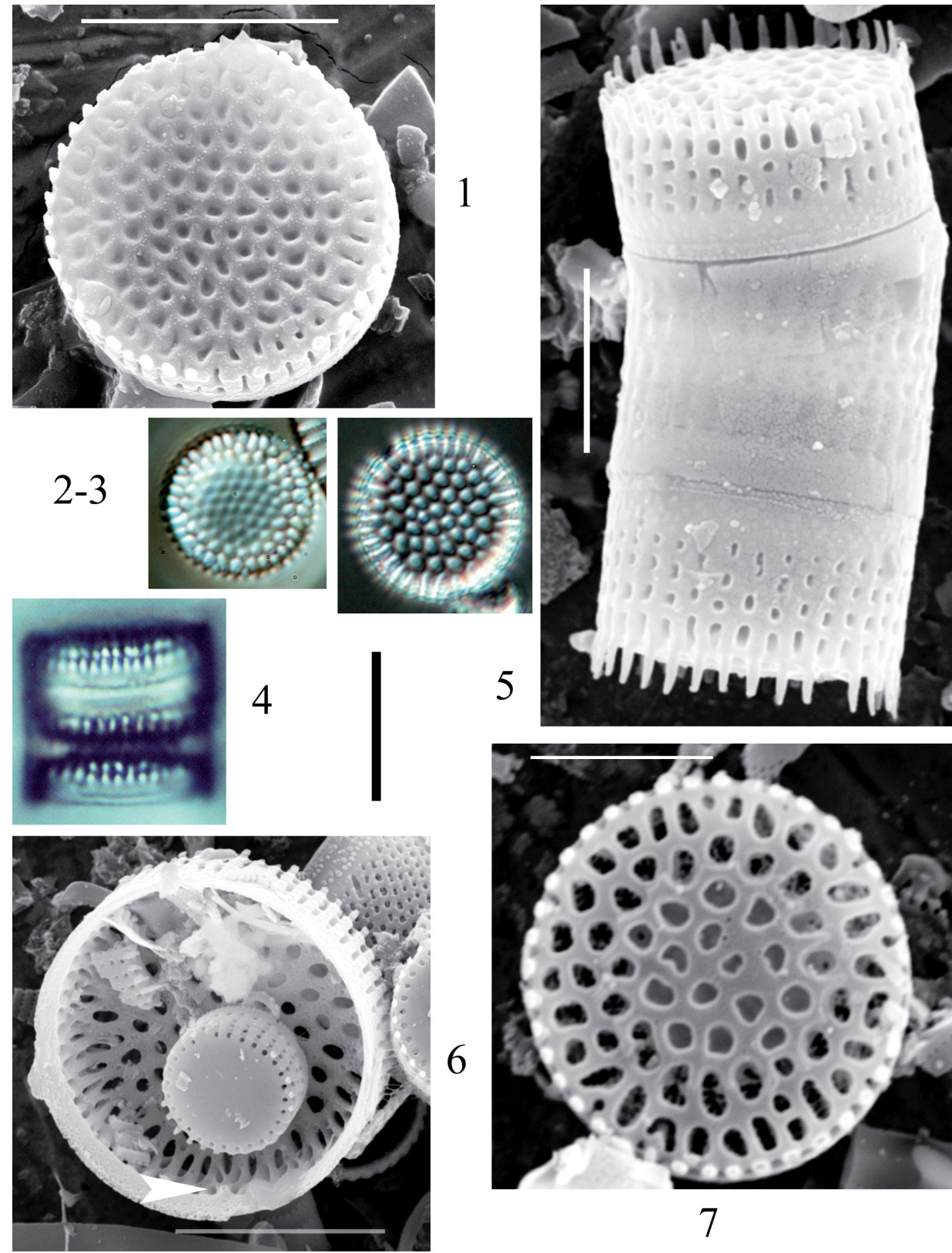

6

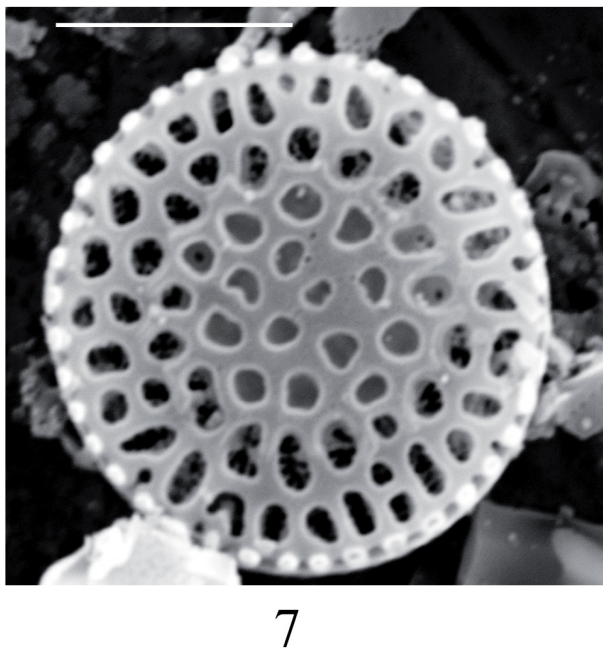

Plate 10: Aulacoseira pfaffiana. - Fig. 1: Lake Brazi, valve side, outside view, SEM. Figs 2-3: Lake Brazi, valve view, LM. Fig. 4: Lake Brazi, mantle view, LM. Fig. 5: Lake Brazi, mantle view, SEM. Fig. 6: Lake Brazi, valve side, inside view (with $A$. alpigena), SEM. Fig. 7: Lake Brazi, valve side, outside view, SEM. Scale bar = $10 \mu \mathrm{m}$ (Figs 1-4, 6), $5 \mu \mathrm{m}$ (Figs 5, 7). 


\section{Aulacoseira valida (Grunow) Krammer 1991: 98 \\ (Plate 11: Figs 1-4)}

References: Krammer (1991), Houk (2003), ENglish and Potapova (2010), BuczKó et al. (2013a).

Distribution in glacial lakes in the Retezat Mountains

\begin{tabular}{ll}
\hline Lakes & $\begin{array}{l}\text { Bucura, Caprelor, Gales, Gemenele, Negru, Peleaga, Slavieu, } \\
\text { Viorica, Zanoaga }\end{array}$ \\
Relative abundance (max.) & $16 \%$ \\
Constancy & $3(43 \%)$ \\
\hline
\end{tabular}

Remarks: PÉTERfi (1993) reported this species from lakes and mires as Melosira italica var. valida. Common, sometimes abundant species in our recent study. 

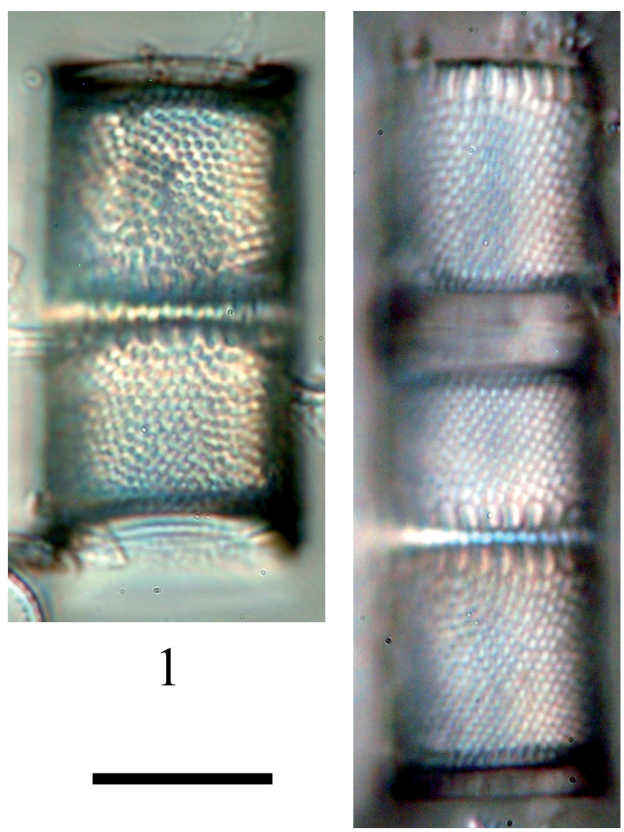

2

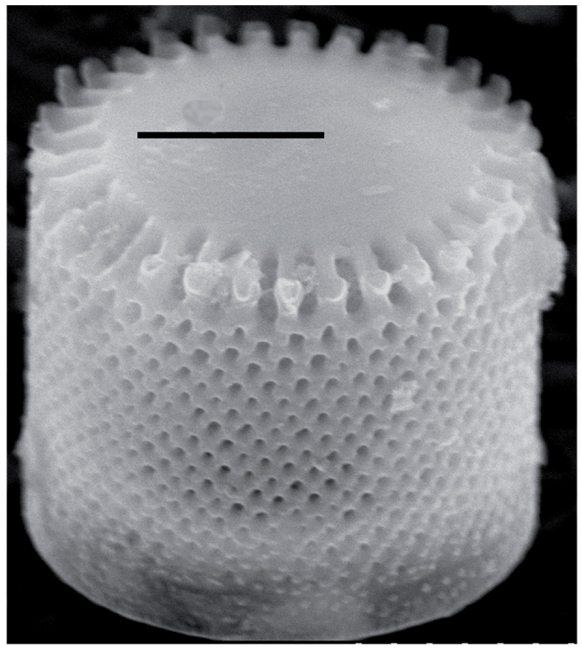

3

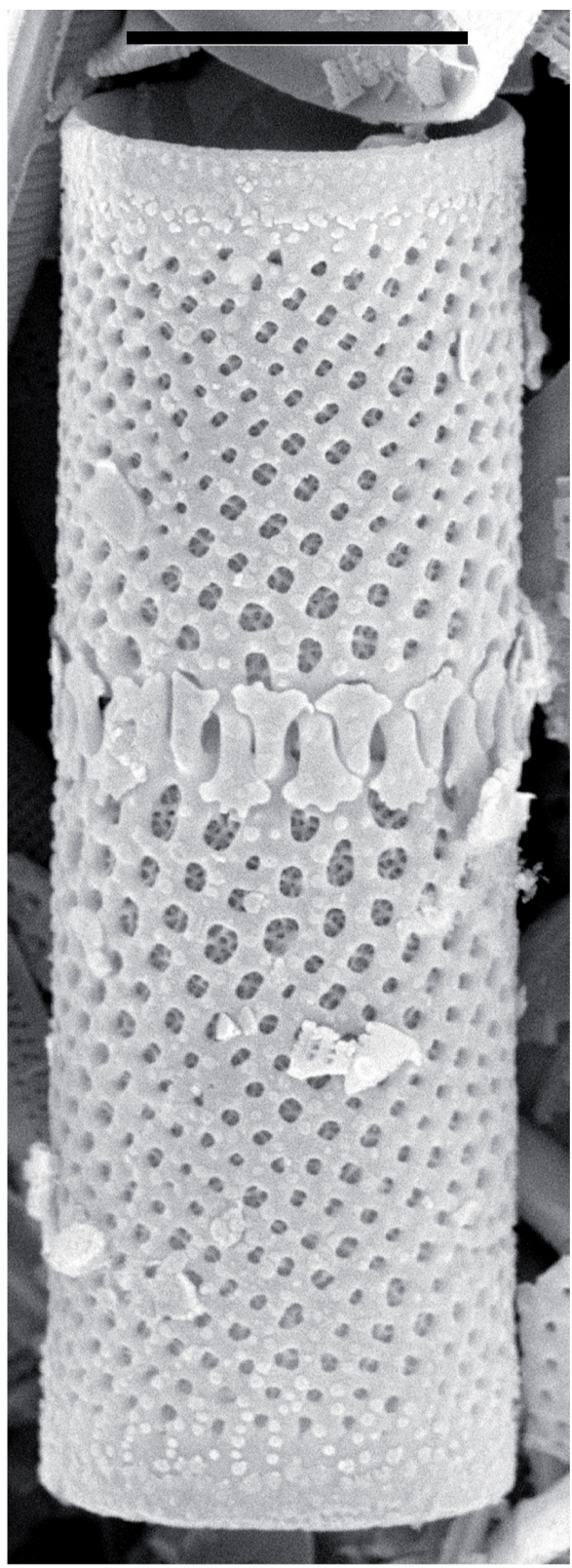

4

Plate 11: Aulacoseira valida. - Figs 1-2: Lake Brazi, LM. Fig. 3: Lake Brazi, oblique view, SEM. Fig. 4: Lake Brazi, mantle view, SEM. Scale bars $=10 \mu \mathrm{m}$. 
Boreozonacola hustedtii Lange-Bertalot, Kulikovskiy et Witkowski in Kulikovskiy, Lange-Bertalot, Witkowski, Dorofeyuk et Genkal 2010: 18 , figs $52: 1-6,53: 1-2$

(Plate 12: Figs 1-7)

References: Krammer and LANGe-Bertalot (1999a as Navicula pseudosilicula Hustedt, p. 169, figs 60: 3-5), Kuli Kovs KiY et al. (2010), BAHLs (2011a).

Distribution in glacial lakes in the Retezat Mountains

\begin{tabular}{ll}
\hline Lakes & Gales, Lia, Stavieu \\
Relative abundance $(\max )$. & $0.5 \%$ \\
Constancy & $1(12 \%)$ \\
\hline
\end{tabular}

Remarks: Probably conspecific with Caloneis ventricosa var. alpina, and it was found in lakes and running waters (PÉTERFI 1993). Rare species in our recent study. 

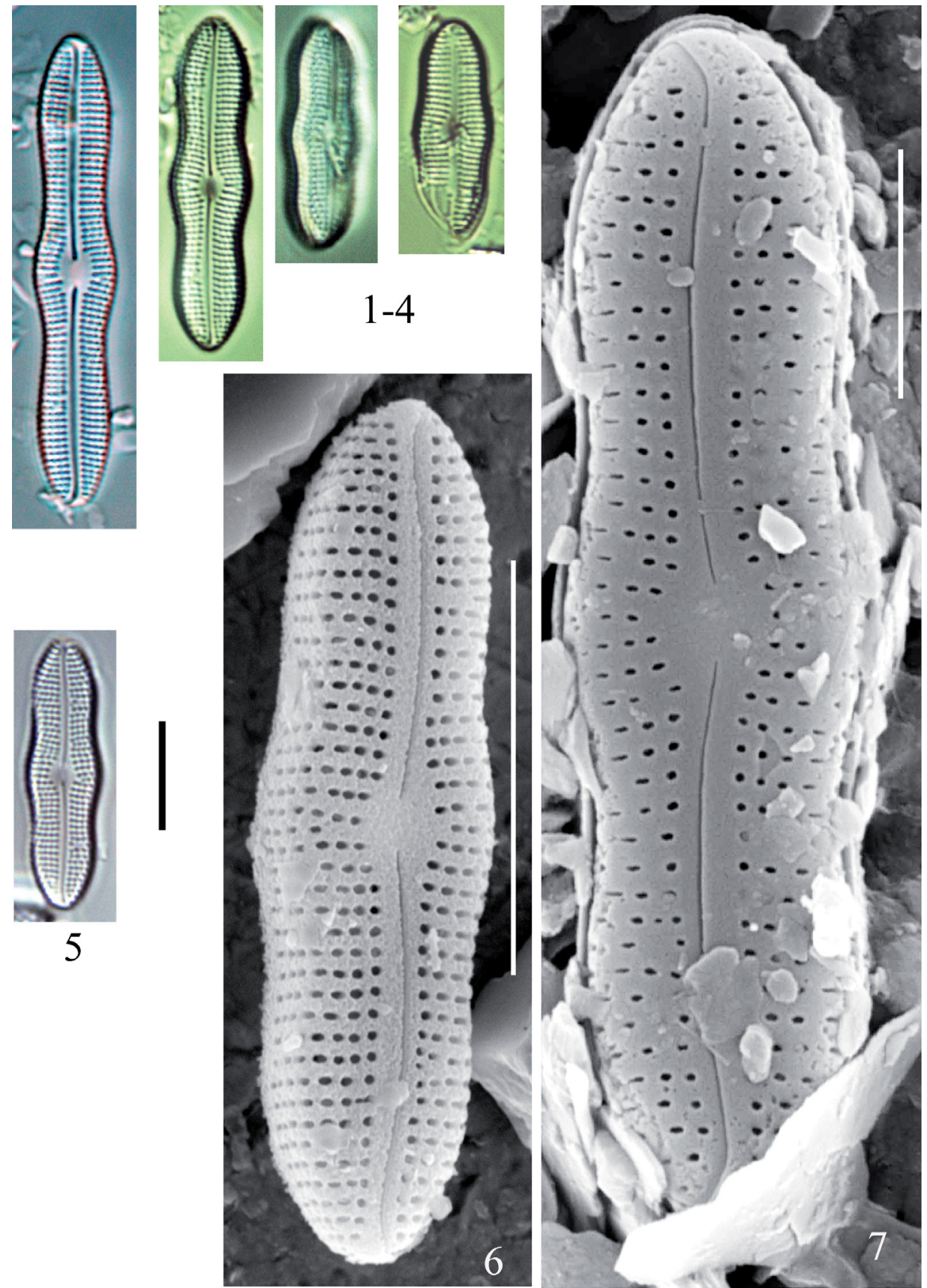

$-6$

-4
-
-
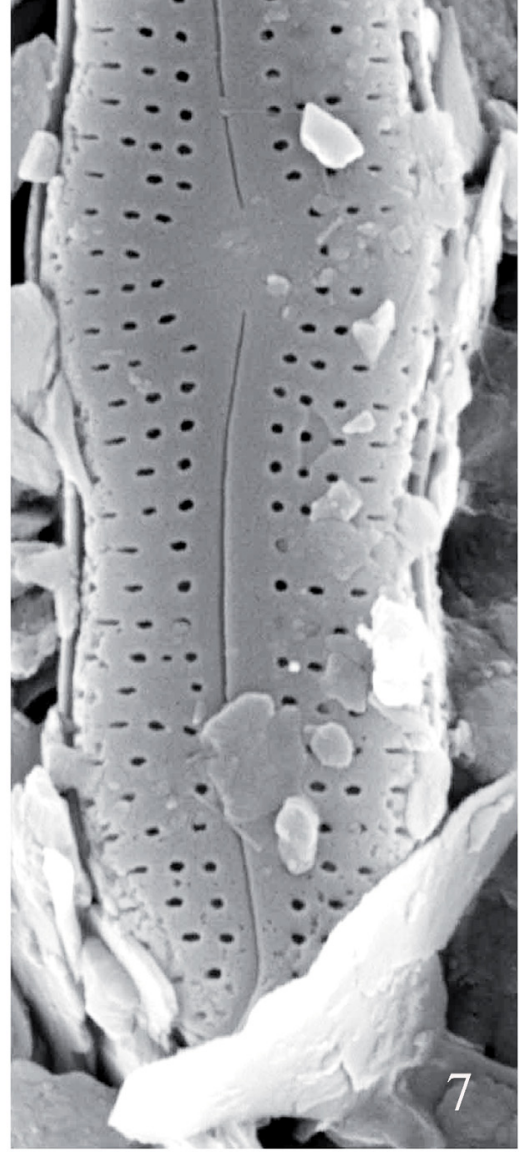

Plate 12: Boreozonacola hustedtii. - Figs 1-4: Lake Gales, LM. Fig. 5: Lake Lia, LM. Fig. 6: Lake Lia, outside view, SEM. Fig. 7: Lake Gales, outside view, SEM. Scale bars $=10 \mu \mathrm{m}$. 
Brachysira brebissonii R. Ross in Hartley, Ross et D. M. Williams 1986: 607

(Plate 13: Figs 1-14)

References: Hartley et al. (1986), LANGe-Bertalot and Moser (1994), HAMiltoN (2010).

Distribution in glacial lakes in the Retezat Mountains

\begin{tabular}{ll}
\hline Lakes & $\begin{array}{l}\text { Brazi, Bucura, Caprelor, Gales, Gemenele, Pietrelice-2, Pietreli- } \\
\text { ce-3, Slavieu, Zanoaga }\end{array}$ \\
Relative abundance (max.) & $1.5 \%$ \\
Constancy & $2(39 \%)$ \\
\hline
\end{tabular}

Remarks: PÉTERfi (1993) reported this species from lakes, mires and running waters, as Anomoeoneis serians var. brachysira. We found it rarely in our recent study. Common species. 

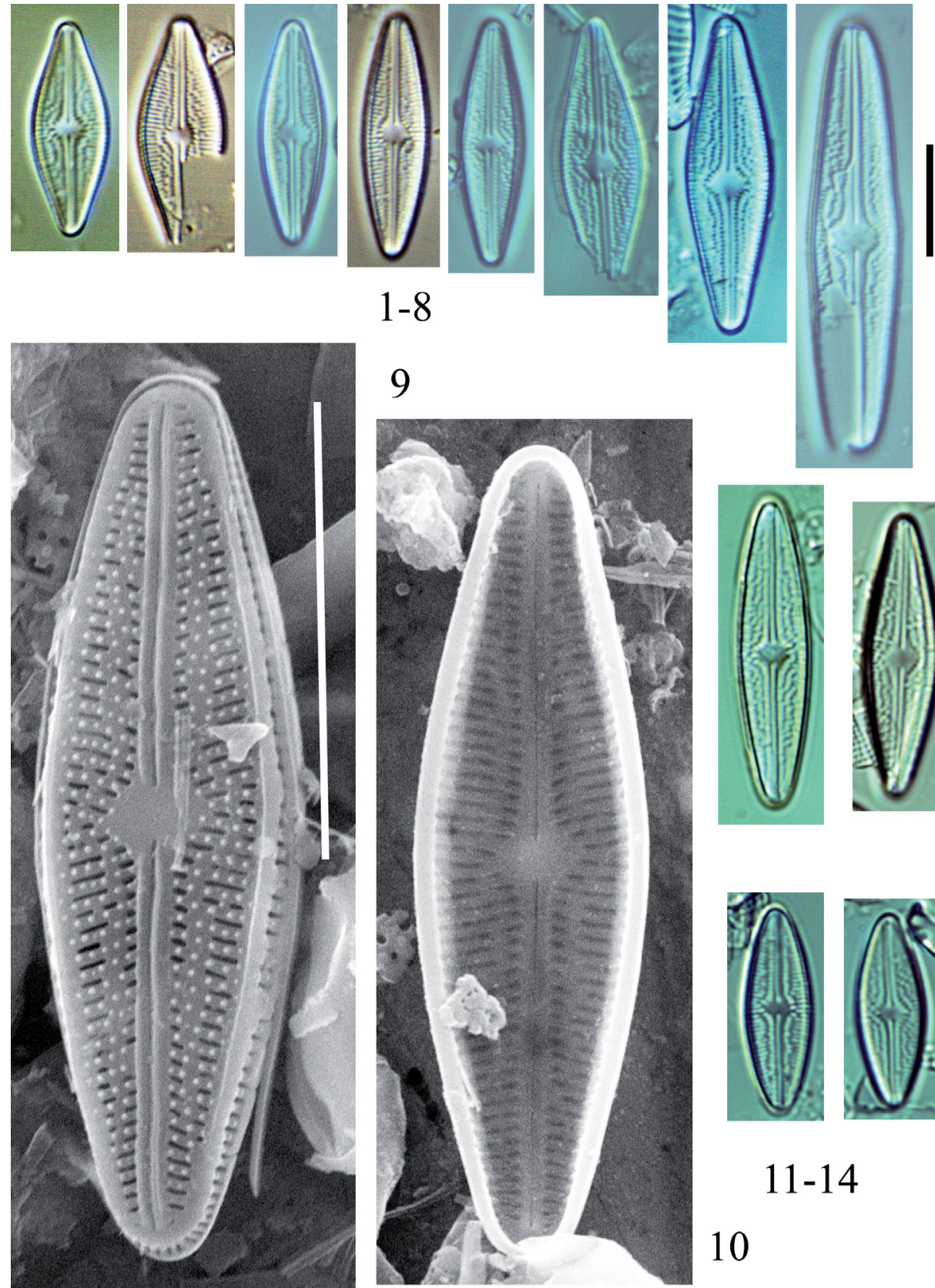

9
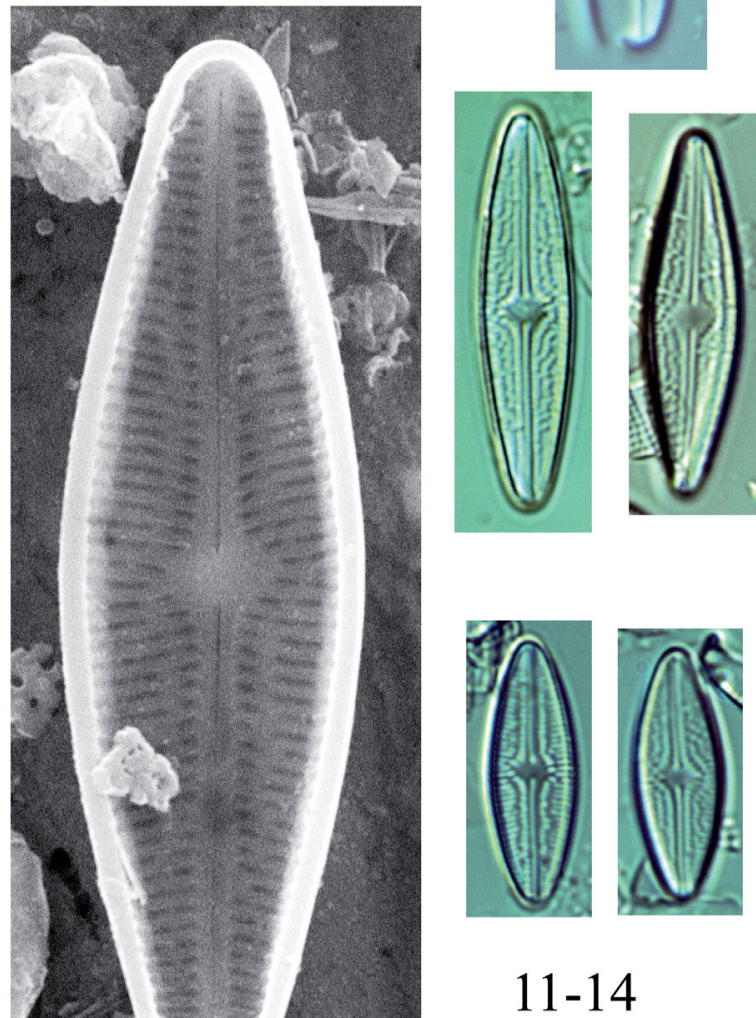

10

Plate 13: Brachysira brebissonii. - Figs 1-8: Lake Slavieu, LM. Fig. 9: Lake Brazi, outside view, SEM. Fig. 10: Lake Slavieu, inside view, SEM. Figs 11-14: Lake Brazi, LM. Scale bars $=10 \mu \mathrm{m}$. 
Cavinula pseudoscutiformis (Hustedt) D. G. Mann et A. J. Stickle in Round, Crawford et Mann 1990: 665

(Plate 14: Figs 1-19)

References: Round et al. (1990), Otu and Spaulding (2011a).

Distribution in glacial lakes in the Retezat Mountains

\begin{tabular}{ll}
\hline Lakes & $\begin{array}{l}\text { Brazi, Caprelor, Florica, Gales, Lezilor, Peleaga, Pietrele, Pietre- } \\
\text { lice-2, Pietrelice-3, Slavieu, Zanoaga }\end{array}$ \\
Relative abundance (max.) & $1.2 \%$ \\
Constancy & $3(48 \%)$ \\
\hline
\end{tabular}

Remarks: PÉTERFi (1993) reported this species from lakes. Common, but never abundant taxon. 


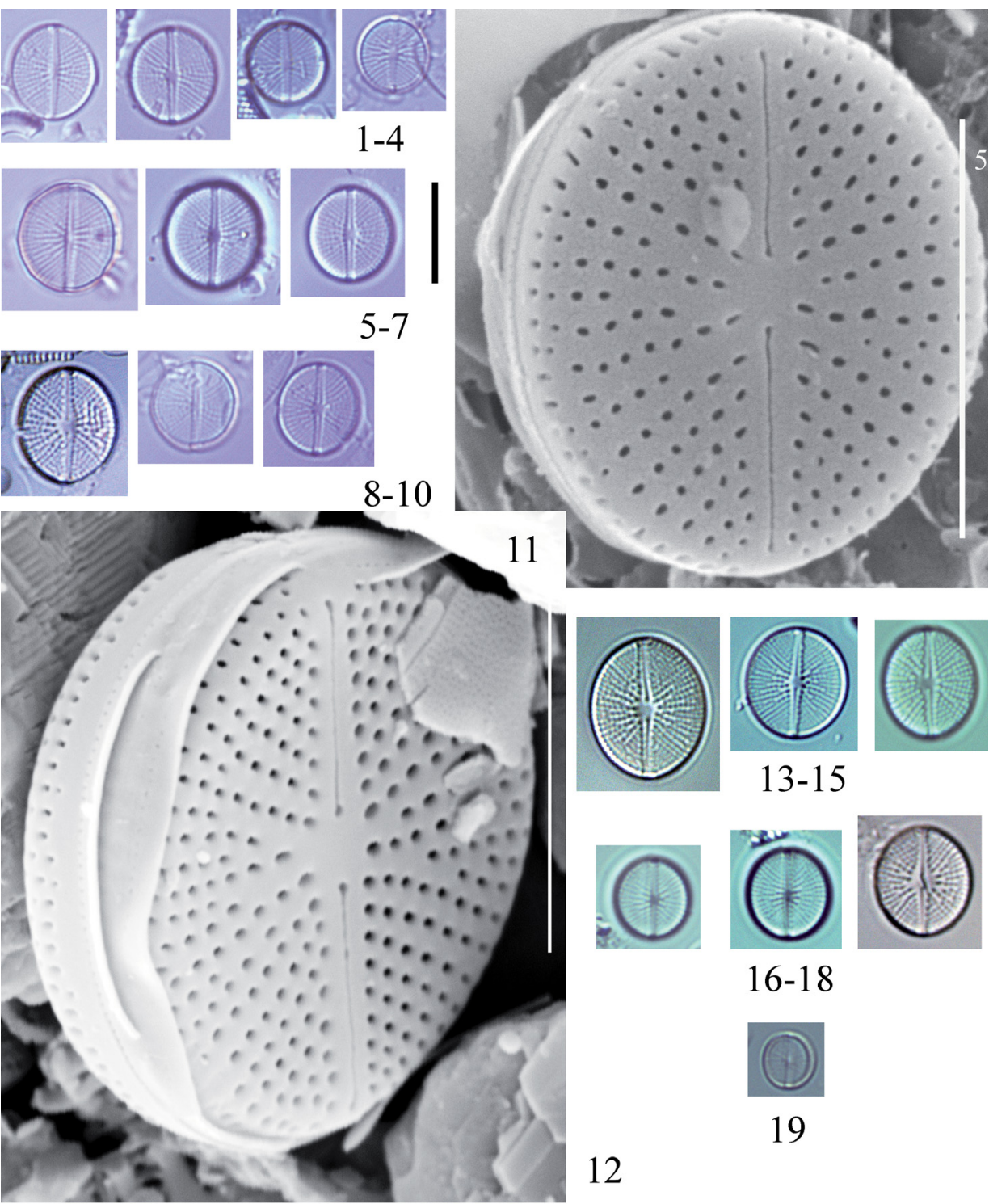

Plate 14: Cavinula pseudoscutiformis. - Figs 1-7: Lake Peleguta, LM. Figs 8-10: Lake Pietrele, LM.

Fig. 11: Lake Peleguta, outside view, SEM. Fig. 12: Lake Brazi, outside view, SEM. Figs 13-19: Lake Brazi, LM. Scale bars $=10 \mu \mathrm{m}$. 
Craticula cf. submolesta (Hustedt) Lange-Bertalot in LangeBertalot et Metzeltin 1996: 42, pl. 104: fig. 1 (Plate 15: Figs 1-10)

References: Lange-Bertalot and Metzeltin (1996), Fallu et al. (2000), LANGe-Bertalot (2001), Siver et al. (2005).

Distribution in glacial lakes in the Retezat Mountains

\begin{tabular}{ll}
\hline Lakes & $\begin{array}{l}\text { Caprelor, Florica, Gemenele, Lezilor, Lia, Peleaga, Peleguta, Ste- } \\
\text { via, Stirbu, Turcelu, Viorica, Zanoaga }\end{array}$ \\
Relative abundance (max.) & $0.5 \%$ \\
Constancy & $3(52 \%)$ \\
\hline
\end{tabular}

Remark: Common species in our recent study. 


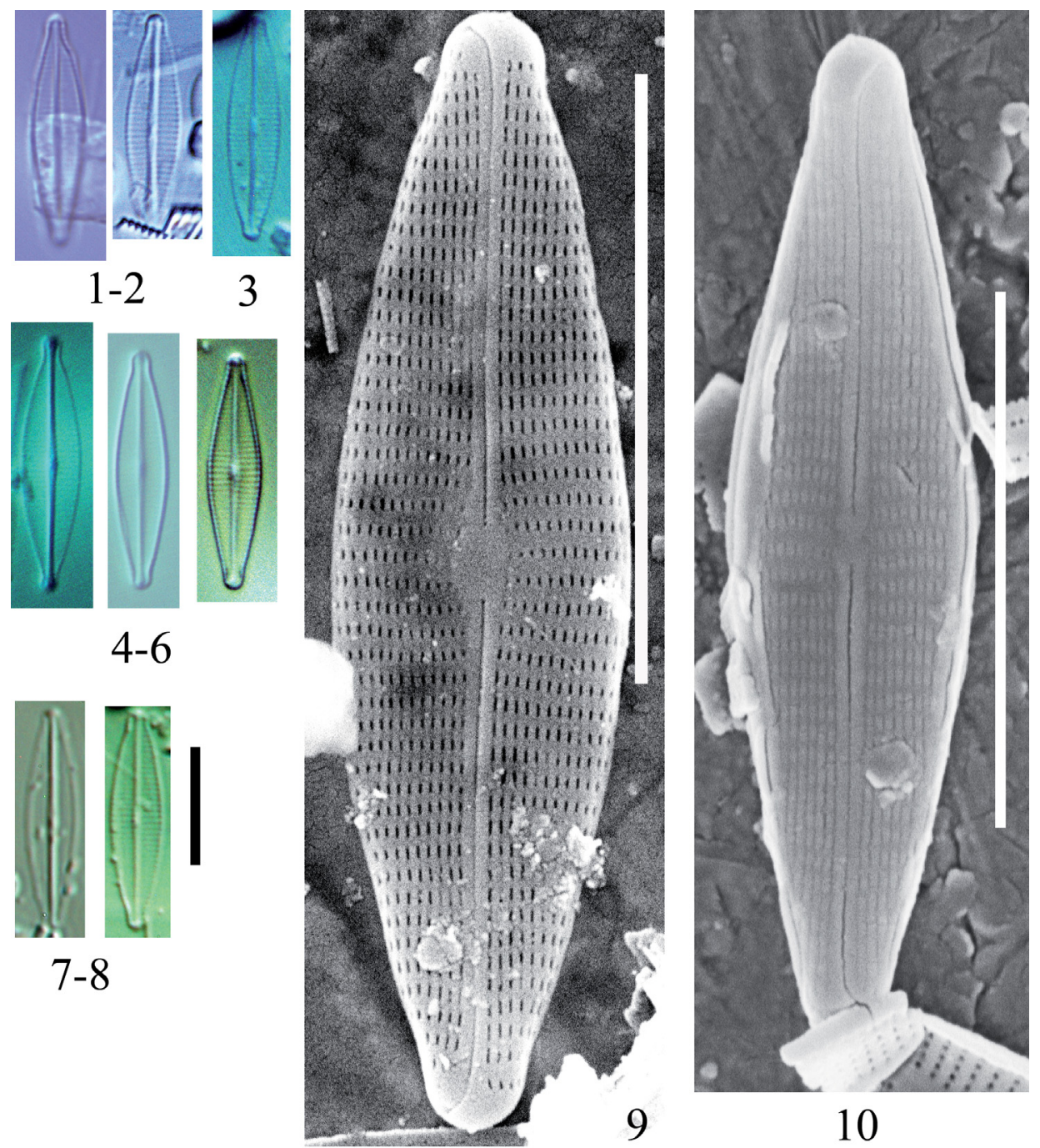

Plate 15: Craticula cf. submolesta. - Figs 1-2: Lake Pietrele, LM. Fig. 3: Lake Peleguta, LM. Figs 4-6: Lake Lezilor, LM. Figs 7-8: Lake Brazi, LM. Fig. 9: Lake Brazi, outside view, SEM. Fig. 10: Lake Pietrele, outside view, SEM. Scale bars $=10 \mu \mathrm{m}$. 
Cymbopleura apiculata Krammer 2003: 12, 152, pl. 7: figs 8-10, pl. 9: figs 1-6, pl. 10: figs 1-4, pl. 11: figs 1-3b (Plate 16: Figs 1-4)

References: Krammer (2003), Bahls (2012a).

Distribution in glacial lakes in the Retezat Mountains

\begin{tabular}{ll}
\hline Lakes & Brazi \\
Relative abundance $(\max )$. & $0.5 \%$ \\
Constancy & $1(4 \%)$ \\
\hline
\end{tabular}

Remark: Very rare species in our recent study. 

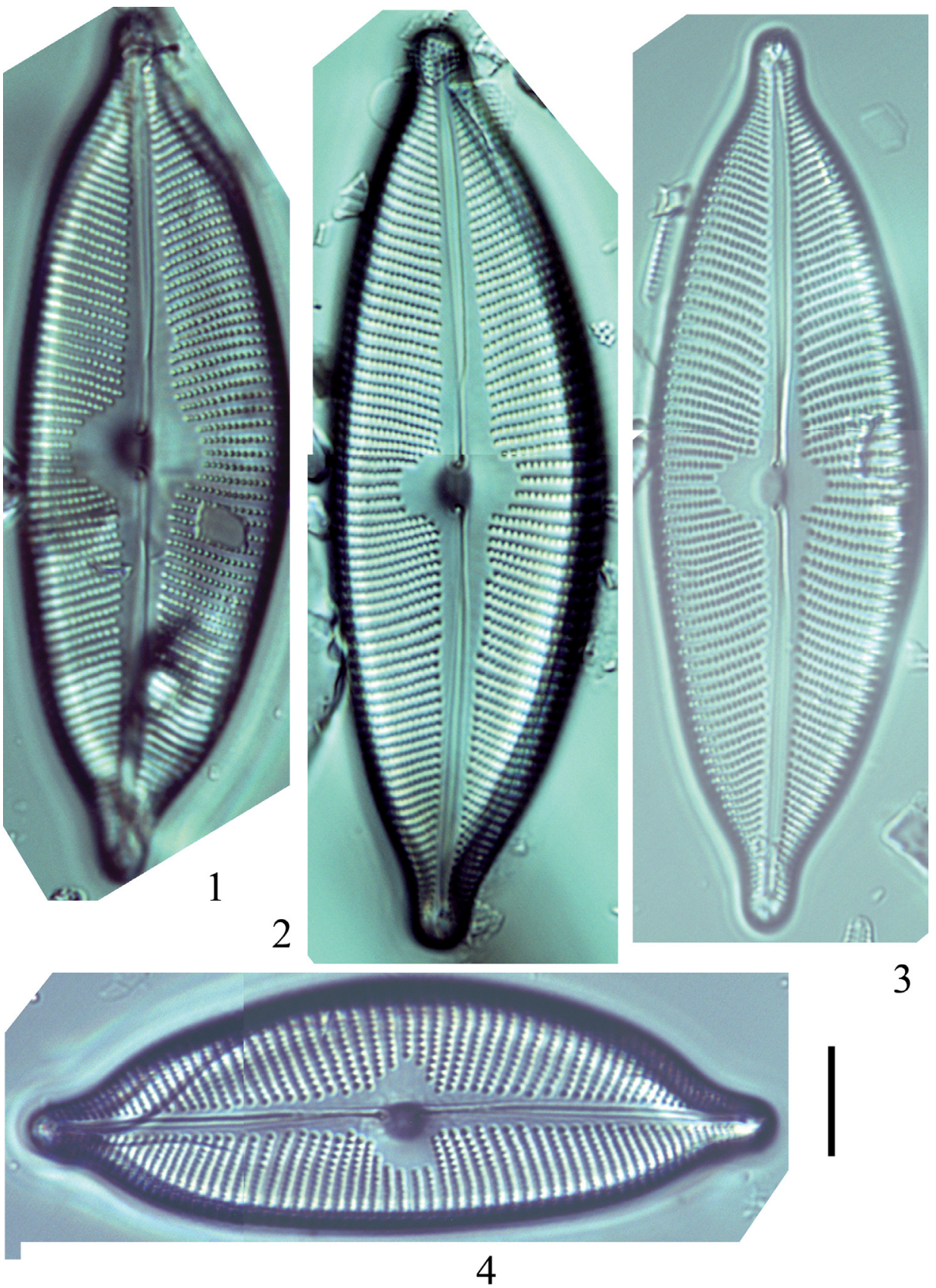

Plate 16: Cymbopleura apiculata. - Figs 1-4: Lake Brazi, LM. Scale bar $=10 \mu \mathrm{m}$. 
Cymbopleura naviculiformis (Auerswald ex Heiberg) Krammer 2003: 56, pl. 76: figs 1-13, pl. 77: figs 1-13, pl. 78: figs 1-8, pl. 79: figs 1-14, pl. 80: fig. 12, pl. 82: figs 1-12, pl. 83: figs 9-11 (Plate 17: Figs 1-11)

References: KRAMmer (2003), BAHLS (2012b).

Distribution in glacial lakes in the Retezat Mountains

\begin{tabular}{ll}
\hline Lakes & Brazi, Lia \\
Relative abundance (max.) & $0.5 \%$ \\
Constancy & $1(8 \%)$ \\
\hline
\end{tabular}

Remarks: PÉTERFI (1993) reported this species from lakes, mires and running waters as Cymbella naviculiformis. This is a rare species in our recent study. 

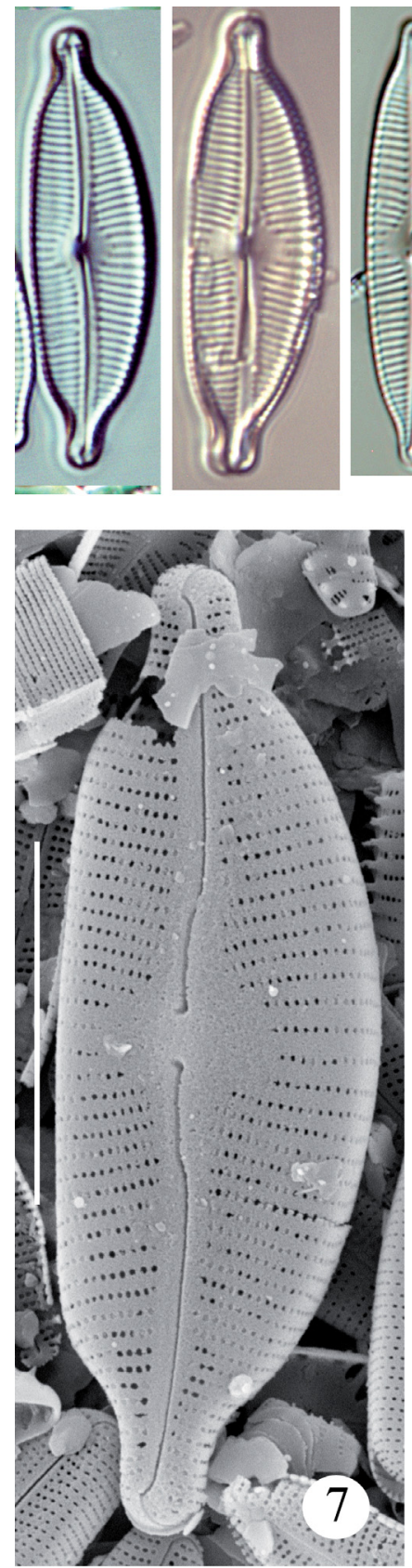
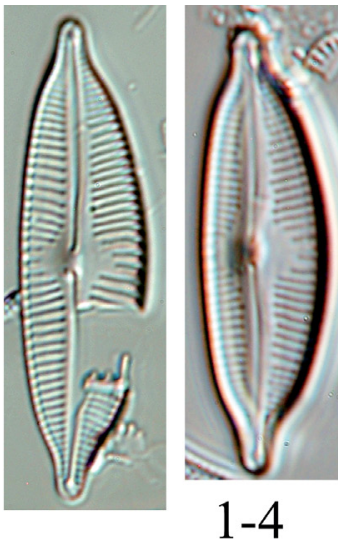

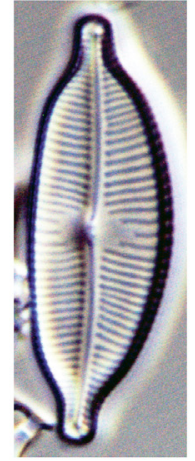

5
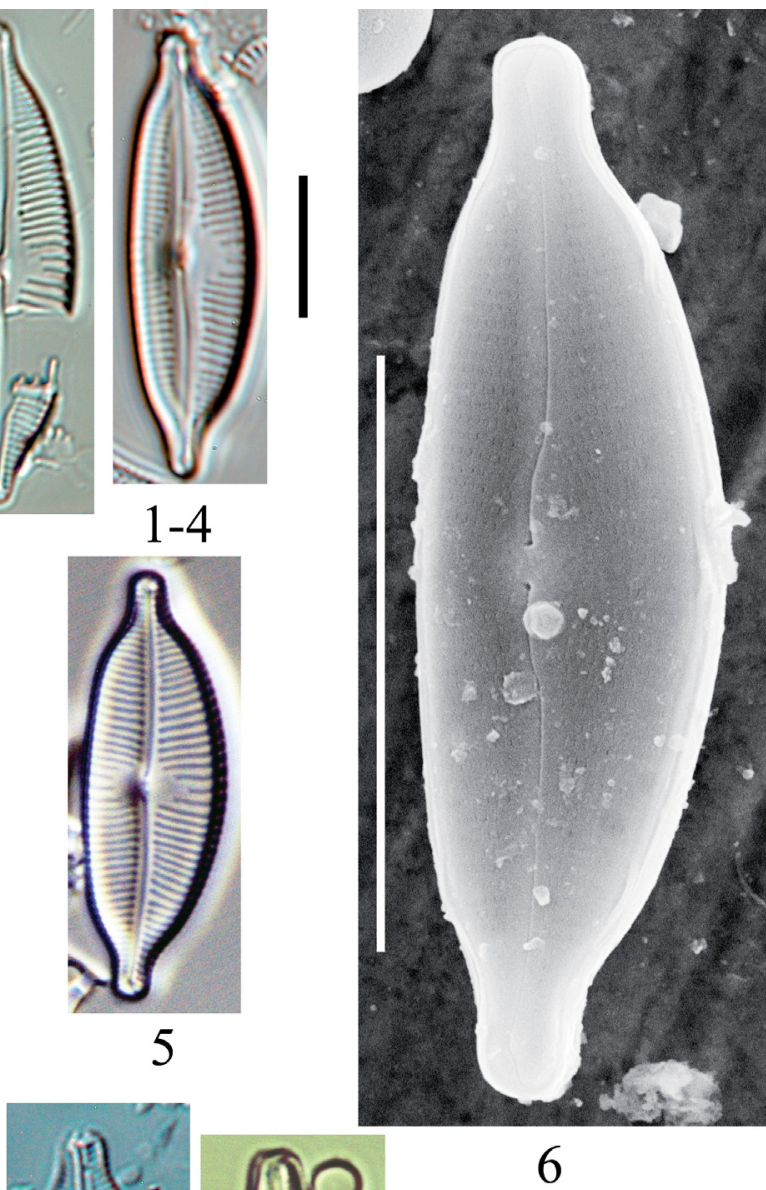

6

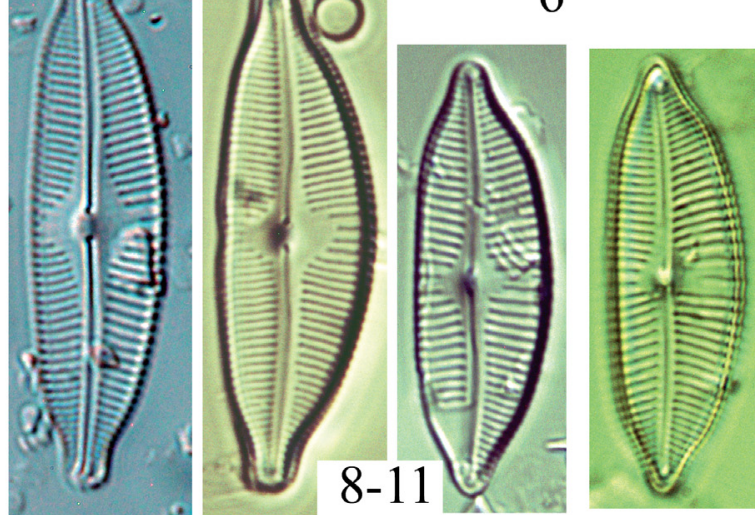

Plate 17: Cymbopleura naviculiformis. - Figs 1-4: Lake Brazi, LM. Fig. 5: Lake Lia, LM. Fig. 6: Lake Brazi, outside view, SEM. Fig. 7: Lake Lia, outside view, SEM. Figs 8-11: Lake Gales, LM. Scale bars $=10 \mu \mathrm{m}$. 
Diatoma mesodon (Ehrenberg) Kützing 1844: 47, pl. 17: fig. XIII [13]

(Plate 18: Figs 1-8)

References: KÜtzING (1844), KRAMMER and LANGe-Bertalot (1991), Potapova (2009c).

Distribution in glacial lakes in the Retezat Mountains

\begin{tabular}{ll}
\hline Lakes & Bucura, Florica, Gales, Gemenele, Lezilor, Lia, Negru, Peleaga, \\
& Pietrele, Pietrelice-2, Pietrelice-3, Slavieu, Stanisoara, Turcelu, \\
& Viorica, Zanoaga \\
Relative abundance (max.) & $3.75 \%$ \\
Constancy & $4(70 \%)$ \\
\hline
\end{tabular}

Remarks: PÉTERfi (1993) reported this species from lakes, mires and running waters as Diatoma hyemale var. mesodon. Fairly common and abundant species in the Retezat Mts. 

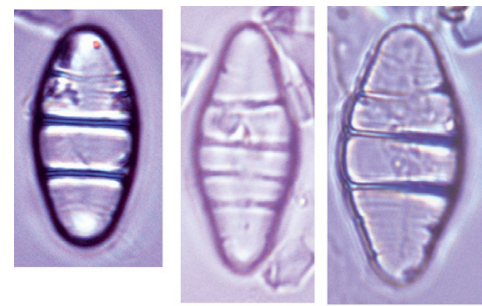

$1-3$

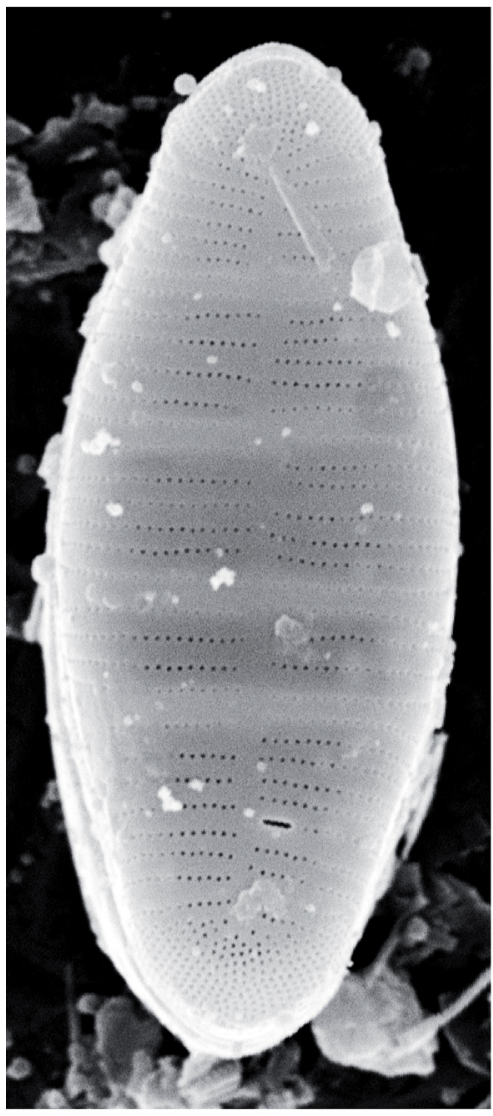

7

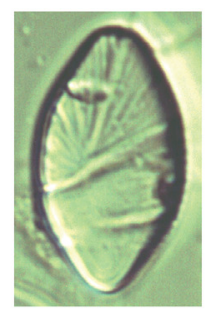

4
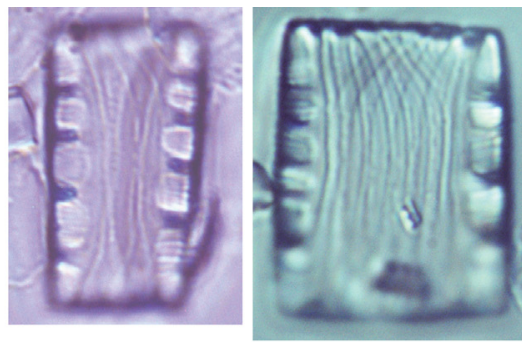

$5-6$

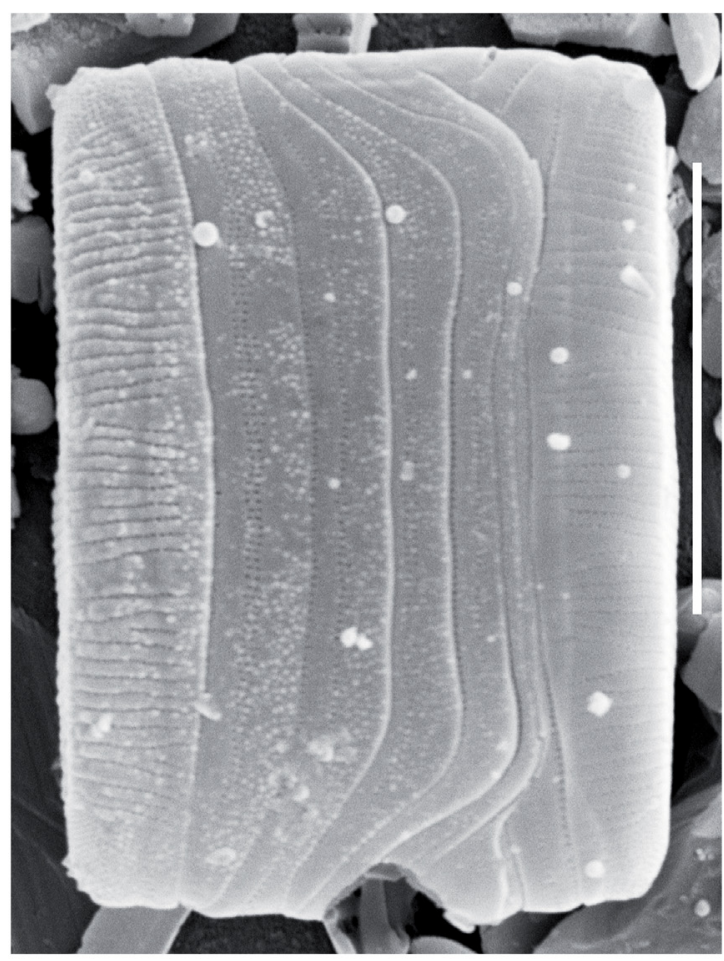

8

Plate 18: Diatoma mesodon. - Figs 1-3: Lake Negru, LM. Fig. 4: Lake Gales, LM. Figs 5-6: Lake Negru, girdle view, LM. Fig. 7: Lake Zanoaga, outside view, SEM. Fig. 8: Lake Zanoaga, girdle view, SEM. Scale bars $=10 \mu \mathrm{m}$. 
Encyonema gaeumannii (Meister) Krammer 1997: 78, pl. 141: figs 1-22, pl. 142: figs 22-23

(Plate 19: Figs 1-15)

Reference: Krammer (1997).

Distribution in glacial lakes in the Retezat Mountains

Lakes Brazi, Bucura, Caprelor, Florica, Gales, Gemenele, Stanisoara, Viorica

Relative abundance $(\max ) \quad 5 \$.

Constancy $2(35 \%)$

Remark: This is a fairly common species in the Retezat Mts. 

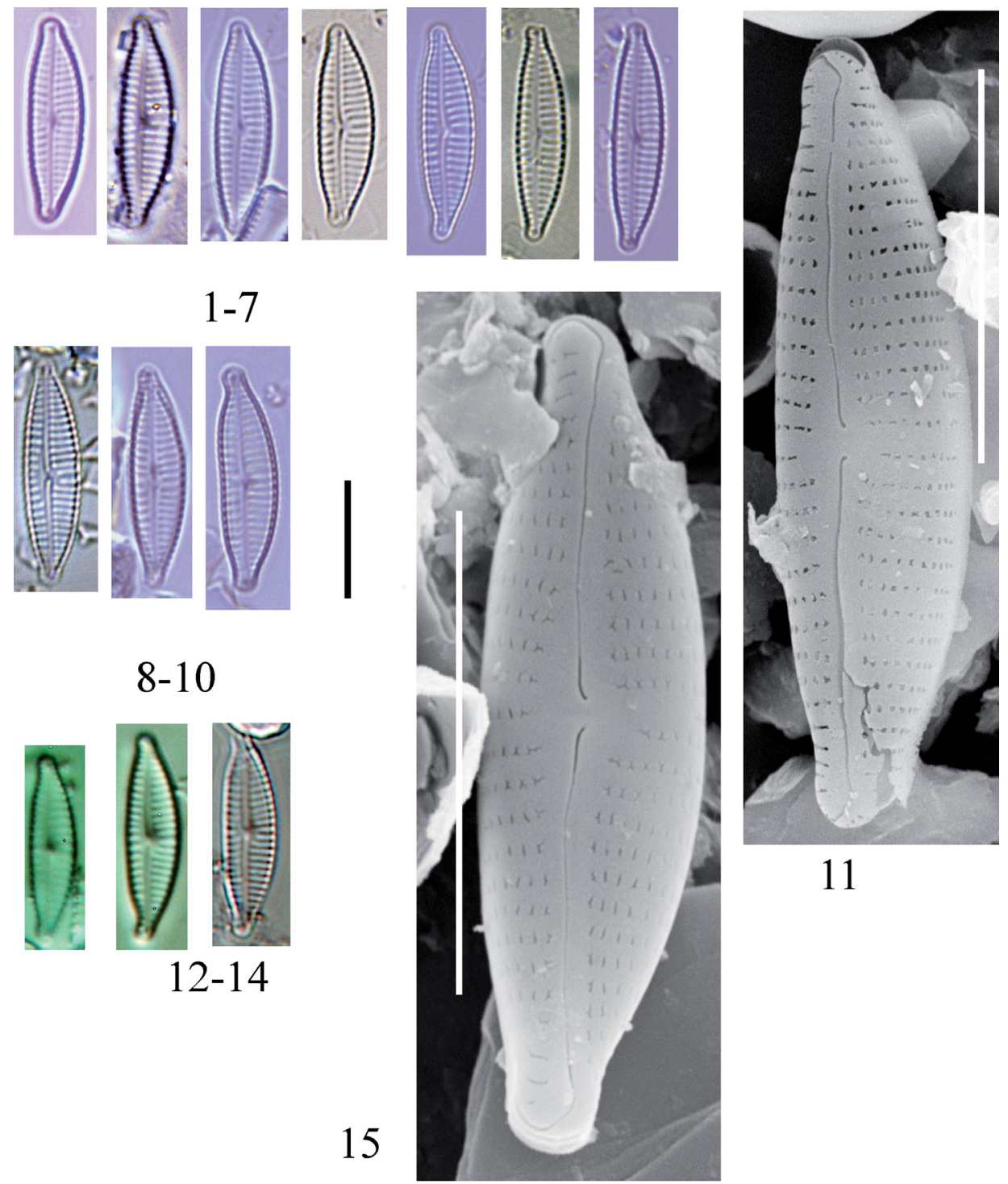

11

Plate 19: Encyonema gaeumannii. - Figs 1-10: Lake Negru, LM. Fig. 11: Lake Negru, outside view, SEM. Figs 12-14: Lake Brazi, LM. Fig. 15: Lake Negru, outside view, SEM. Scale bars $=10 \mu \mathrm{m}$. 
Encyonema neogracile Krammer 1997: 177, pl. 82: figs 1-13, pl. 83: figs 7-10, pl. 86: figs 9-12, pl. 90: fig. 6, pl. 91: figs 1-2 (Plate 20: Figs 1-12)

Reference: Krammer (1997), Hofmann et al. (2013).

Distribution in glacial lakes in the Retezat Mountains

\begin{tabular}{ll}
\hline Lakes & $\begin{array}{l}\text { Ana, Brazi, Bucura, Caprelor, Gemenele, Lezilor, Peleaga, Viori- } \\
\text { ca, Zanoaga }\end{array}$ \\
Relative abundance (max.) & $6 \%$ \\
Constancy & $2(39 \%)$ \\
\hline
\end{tabular}

Remark: PÉteRfi (1993) reported this species from lakes and mires as Cymbella gracilis. 

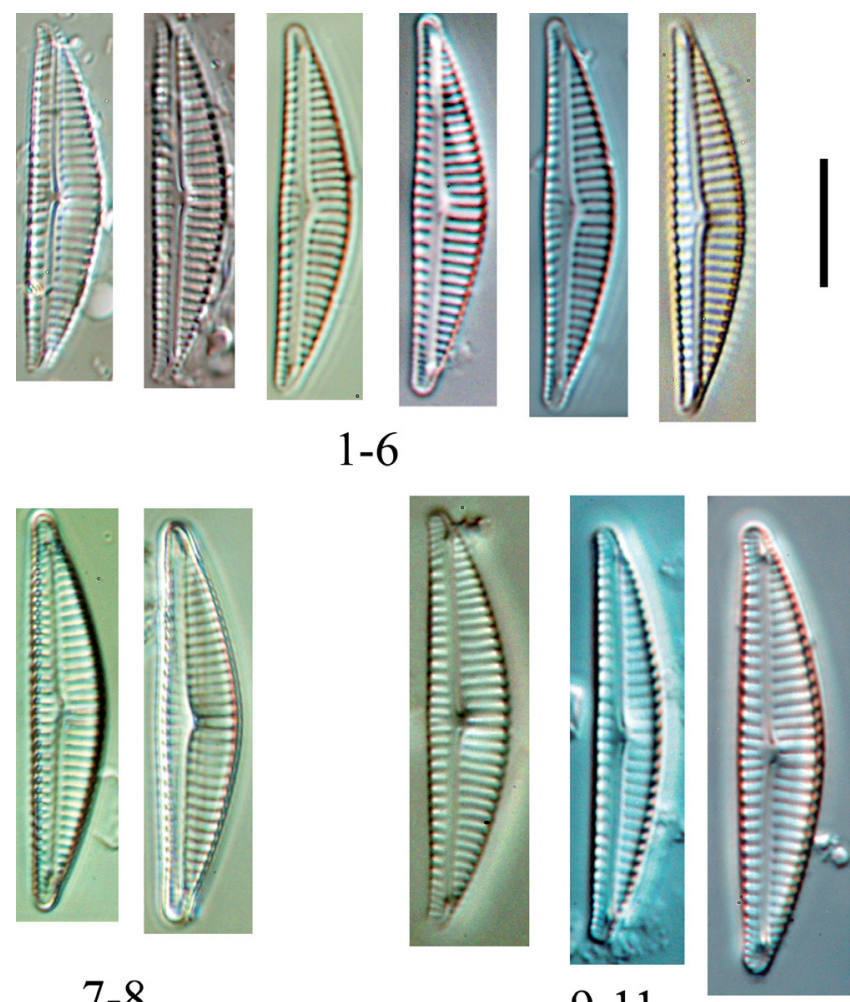

$7-8$
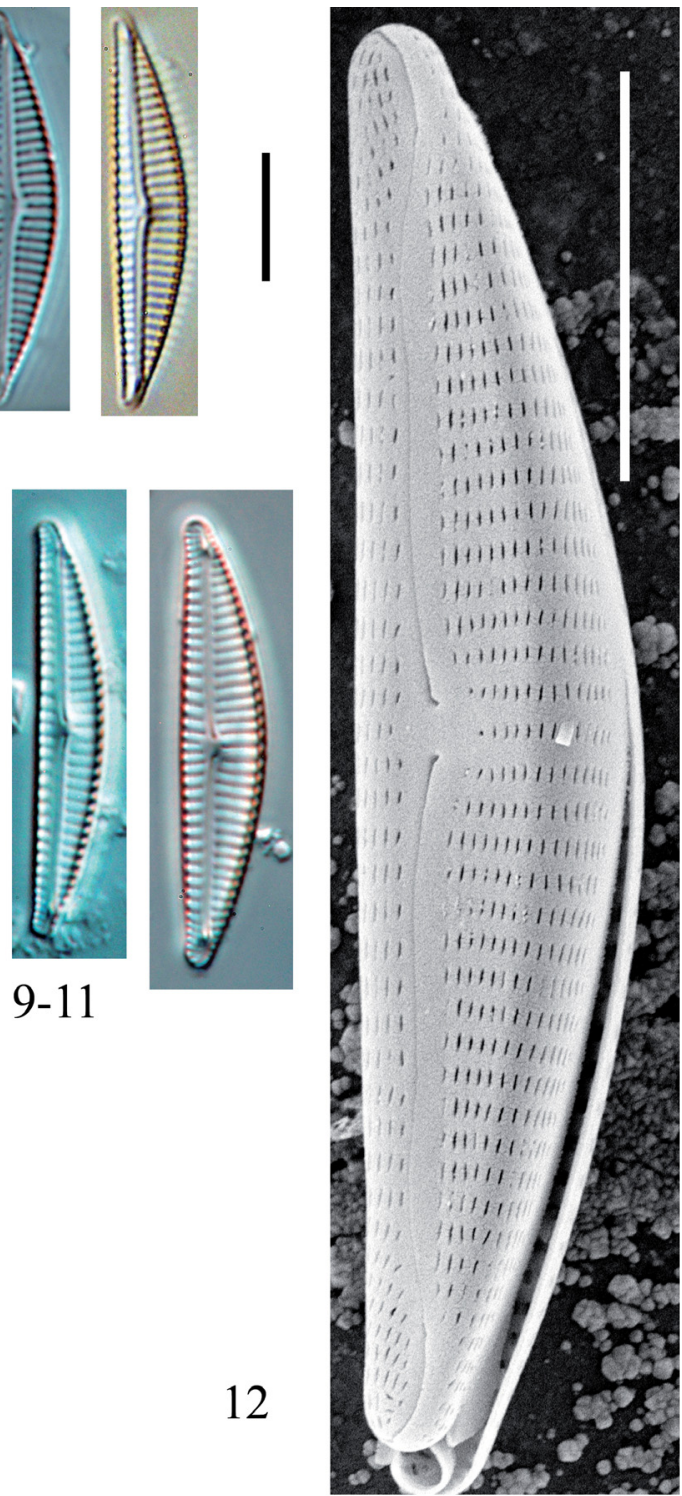

Plate 20: Encyonema neogracile. - Figs 1-8: Lake Brazi, LM. Figs 9-11: Lake Gales, LM. Fig. 12: Lake Brazi, outside view, SEM. Scale bars $=10 \mu \mathrm{m}$. 
Encyonema perpusillum (Cleve-Euler) D. G. Mann in Round, R. M. Crawford et D. G. Mann 1990: 667

(Plate 21: Figs 1-5)

References: Round et al. (1990), Hofmann et al. (2013).

Distribution in glacial lakes in the Retezat Mountains

\begin{tabular}{ll}
\hline Lakes & Zanoaga \\
Relative abundance (max.) & $0.5 \%$ \\
Constancy & $1(4 \%)$ \\
\hline
\end{tabular}

Remarks: PÉTERFI (1993) reported this species from mires as Cymbellaperpusilla. Very rare species in our recent study. 

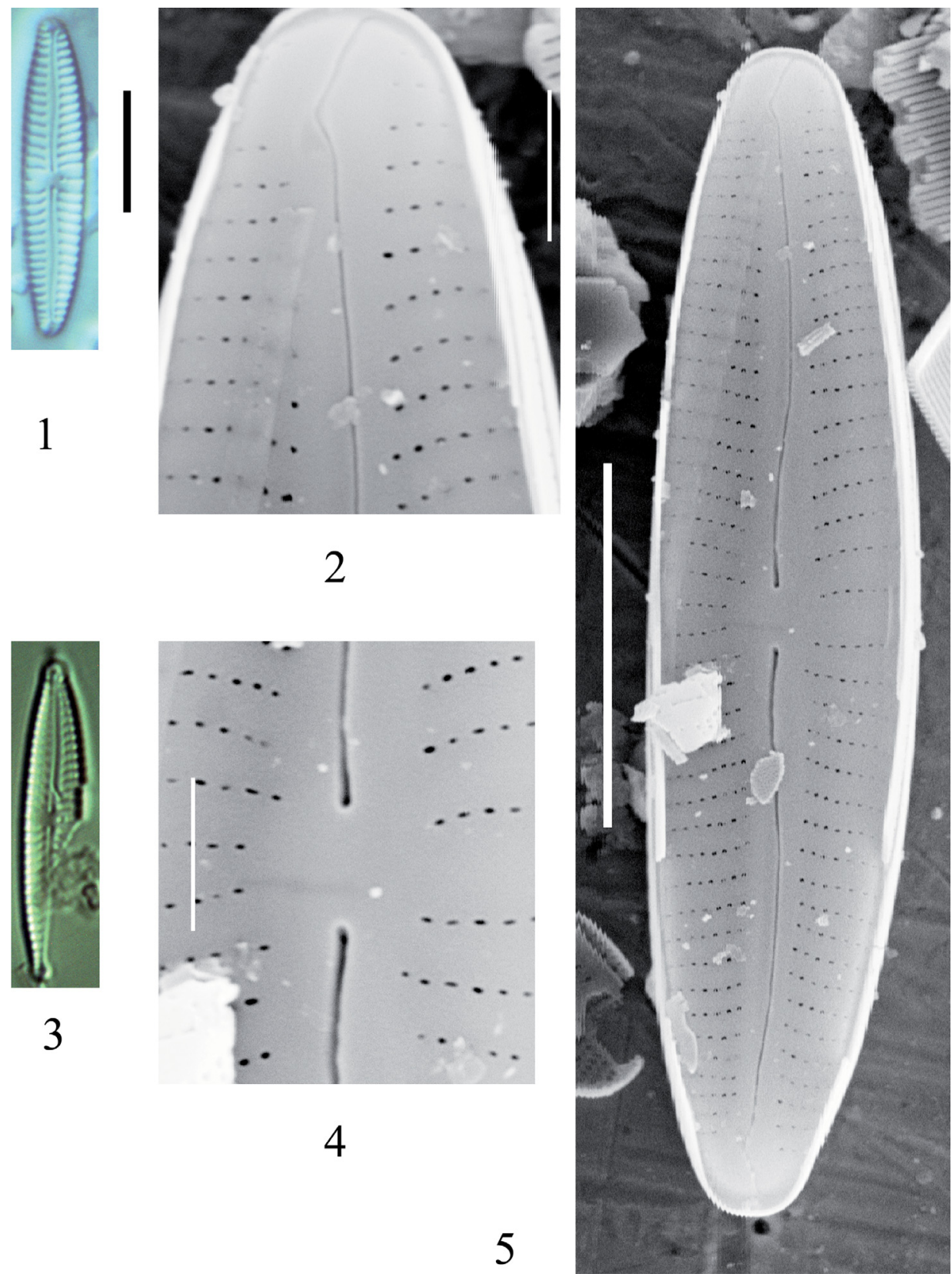

4

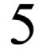

Plate 21: Encyonema perpusillum. - Figs 1, 3: Lake Zanoaga, LM. Fig. 2: Lake Zanoaga, apices, outside view, SEM. Fig. 4: Lake Zanoaga, central area, outside view, SEM. Fig. 5: Lake Zanoaga, outside view, SEM. Scale bars $=10 \mu \mathrm{m}$. 
Encyonema silesiacum (Bleisch) D. G. Mann in Round, R. M. Crawford et D. G. Mann 1990: 667

(Plate 22: Figs 1-11)

References: Round et al. (1990), KRAMMER and LANGE-BERTALOT (1991).

Distribution in glacial lakes in the Retezat Mountains

Lakes

Ana, Brazi, Bucura, Florica, Lezilor, Lia, Peleguta, Pietrele, Pietrelice-1, Pietrelice-2, Pietrelice-3, Slavieu, Stanisoara, Viorica

Relative abundance (max.) $\quad 3 \%$

Constancy $3(61 \%)$

Remark: Common, but rarely abundant species in the Retezat Mts. 

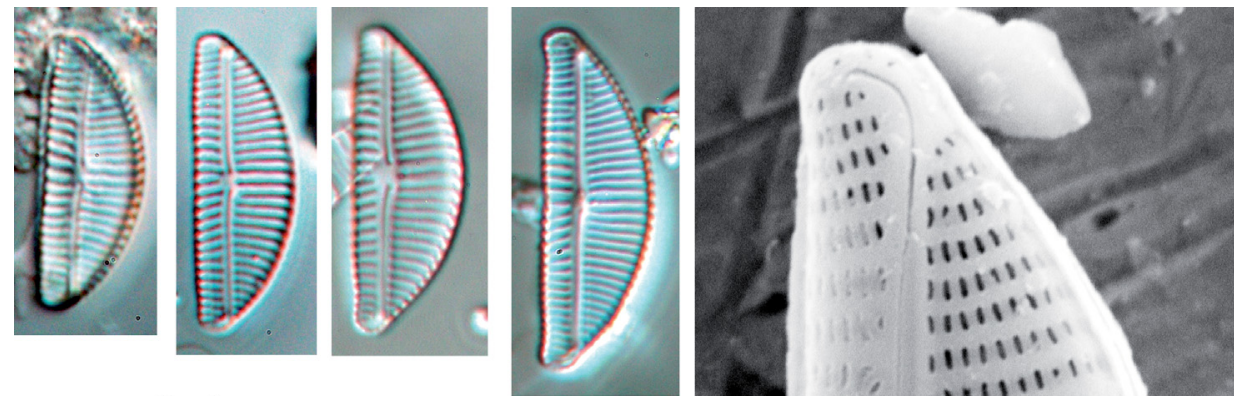

$1-4$
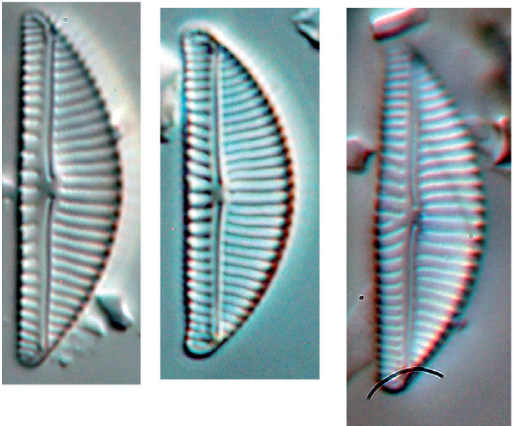

\section{$5-7$}
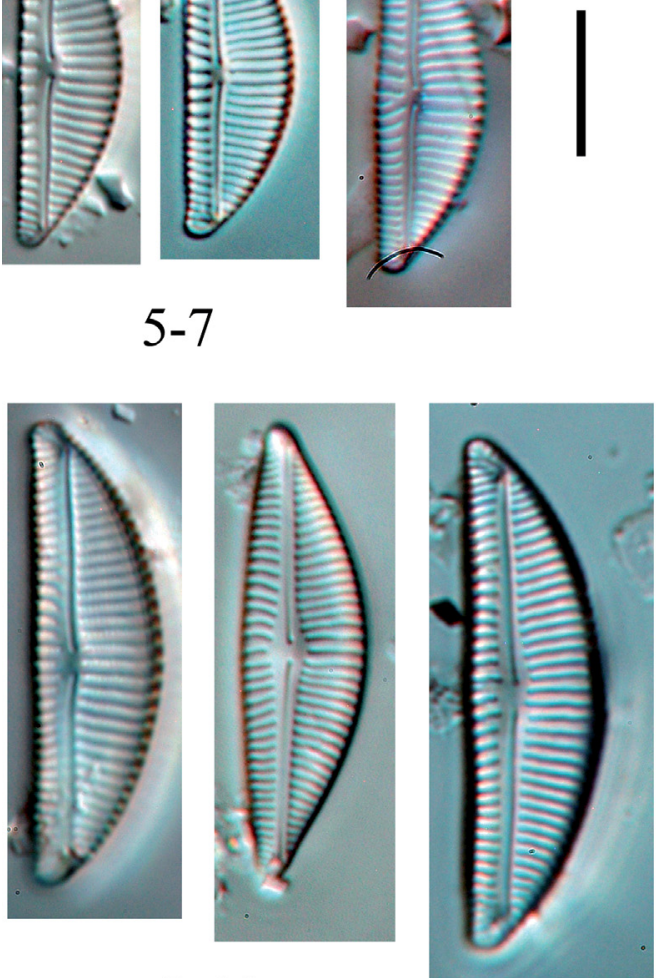

\section{$8-10$}

Plate 22: Encyonema silesiacum. - Figs 1-10: Lake Gales, LM. Fig. 11: Lake Gales, outside view, SEM. Scale bars $=10 \mu \mathrm{m}$. 
Fragilaria gracilis Østrup 1910: 190, pl. V: fig. 117 (Plate 23: Figs 1-24)

References: ØStRuP (1910), KRAMMER and LANGE-BERTALOt (1991).

\section{Distribution in glacial lakes in the Retezat Mountains}

\begin{tabular}{ll}
\hline Lakes & Ana, Bucura, Gales, Lia, Negru, Zanoaga \\
Relative abundance (max.) & $4 \%$ \\
Constancy & $2(26 \%)$ \\
\hline
\end{tabular}

Remark: Fairly common, sometimes abundant species. 

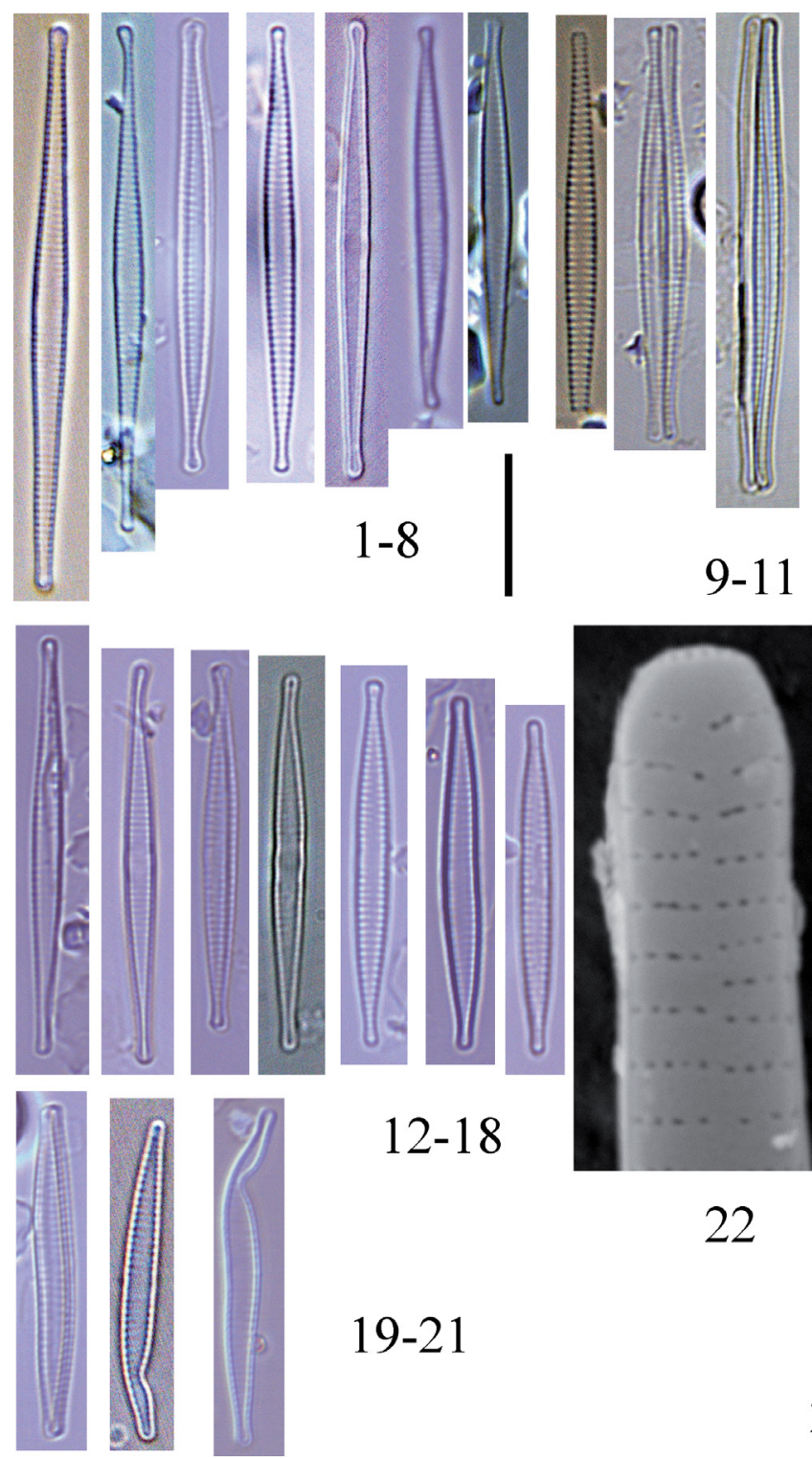

$12-18$
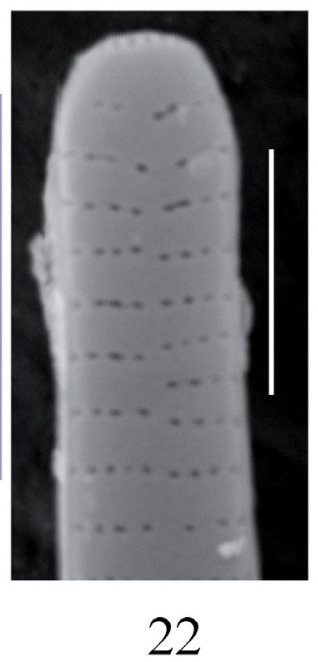

$19-21$
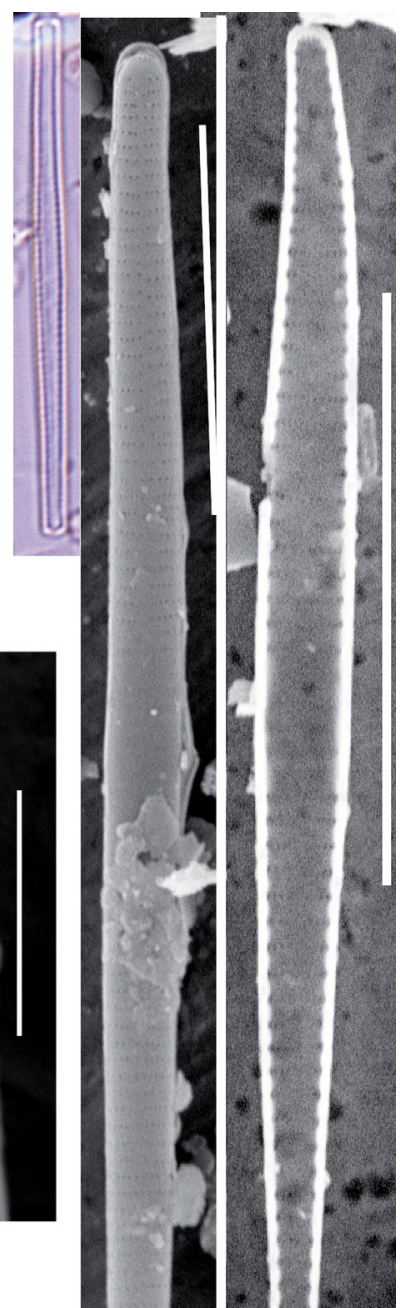
Fragilariforma bicapitata (Mayer) D. M. Williams et Round 1988: 265

(Plate 24: Figs 1-13)

References: Williams and Round (1988b), Bishop (2014).

Distribution in glacial lakes in the Retezat Mountains

\begin{tabular}{ll}
\hline Lakes & Lia, Viorica \\
Relative abundance (max.) & $0.5 \%$ \\
Constancy & $1(4 \%)$ \\
\hline
\end{tabular}

Remark: Very rare species in our recent study. 

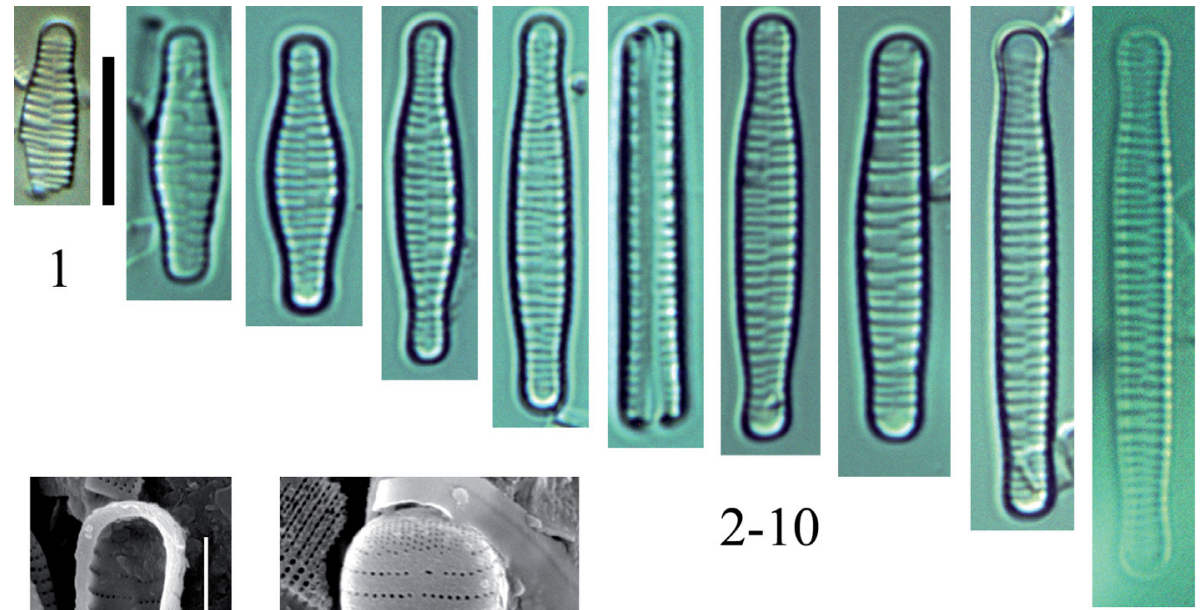

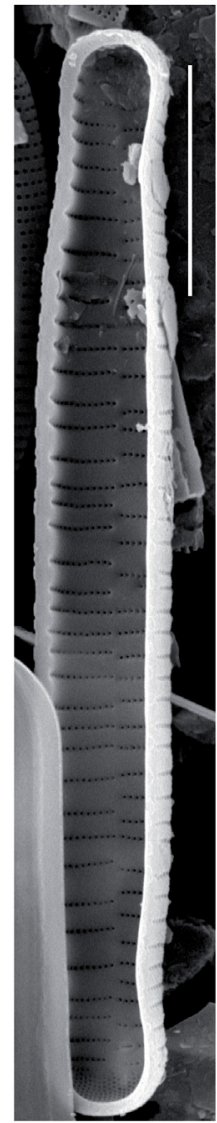

11

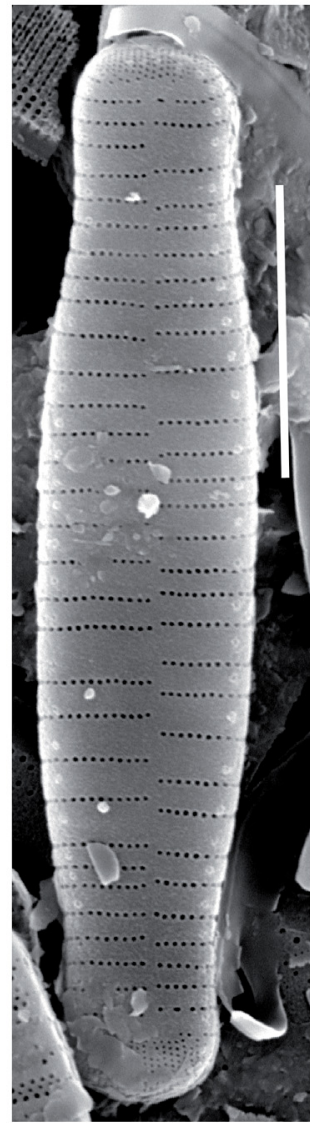

12

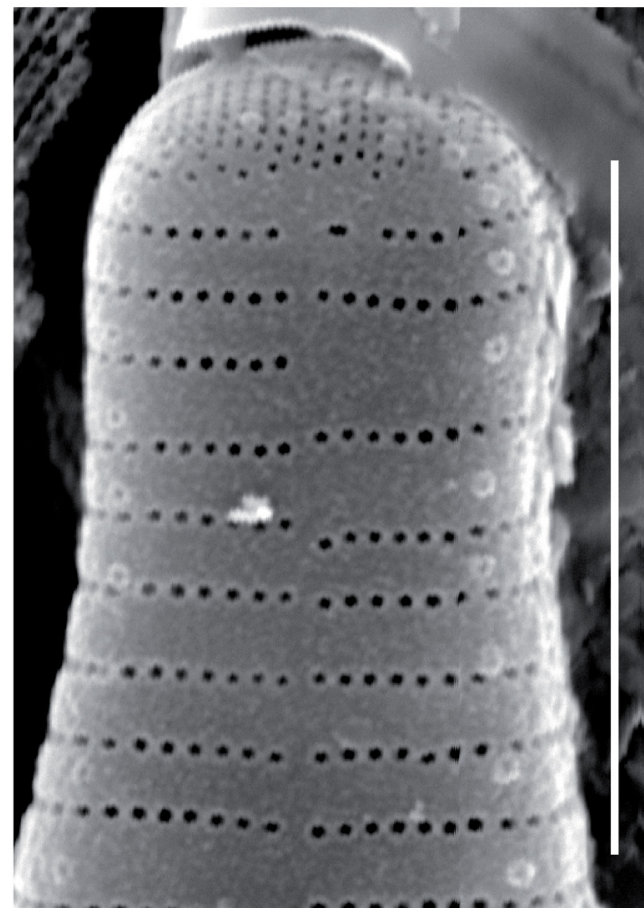

13

Plate 24. Fragilariforma bicapitata. - Fig. 1: Lake Viorica, LM. Figs 2-10: Lake Lia, LM. Fig. 11 : Lake Lia, inside view, SEM. Figs 12-13: Lake Lia, outside view, SEM. Scale bar $=10 \mu \mathrm{m}$ (Figs 1-10), $2 \mu \mathrm{m}$ (Figs 11-13). 
Fragilariforma virescens (Ralfs) D. M. Williams et Round 1988: 265

(Plate 25: Figs 1-12)

References: Williams and Round (1988b), Morales and Spaulding (2011).

Distribution in glacial lakes in the Retezat Mountains

\begin{tabular}{ll}
\hline Lakes & $\begin{array}{l}\text { Bucura, Gales, Lezilor, Negru, Pietrelice-2, Pietrelice-3, Slavieu, } \\
\text { Turcelu, Zanoaga }\end{array}$ \\
Relative abundance $(\max )$. & $2 \%$ \\
Constancy & $2(39 \%)$ \\
\hline
\end{tabular}

Remarks: PÉTERFI (1993) reported this species from lakes, mires and running waters as Fragilaria virescens, and $F$. virescens var. elliptica. Common, but not abundant taxon in the Retezat Mts. 


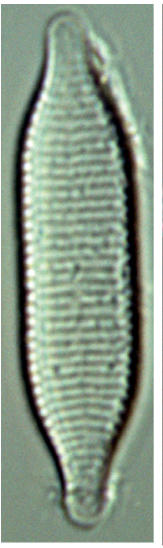

1
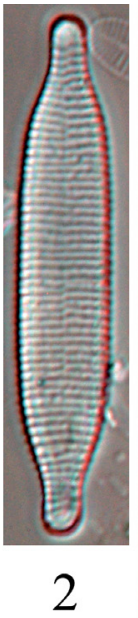

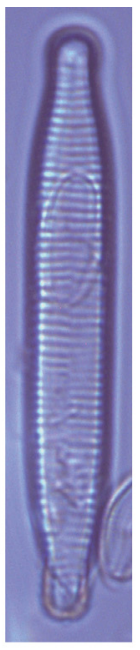

3

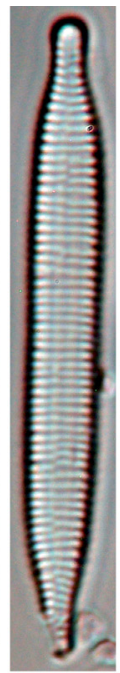

4

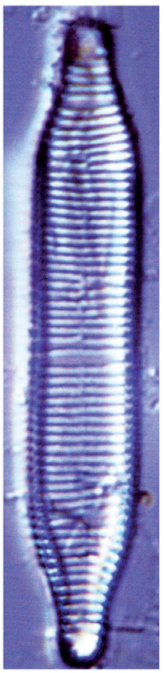

5
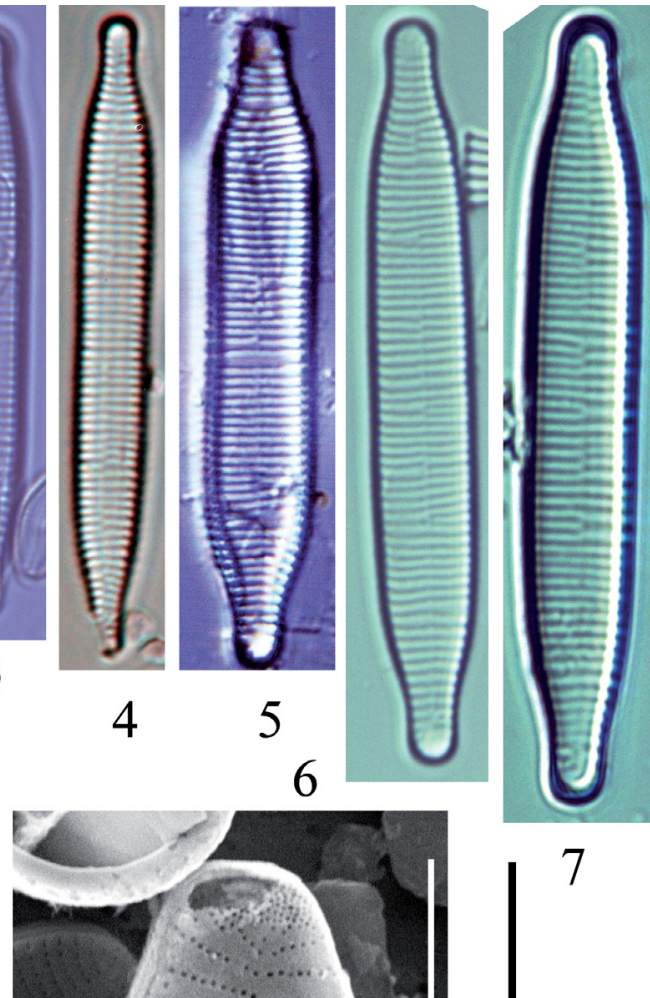

3

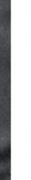
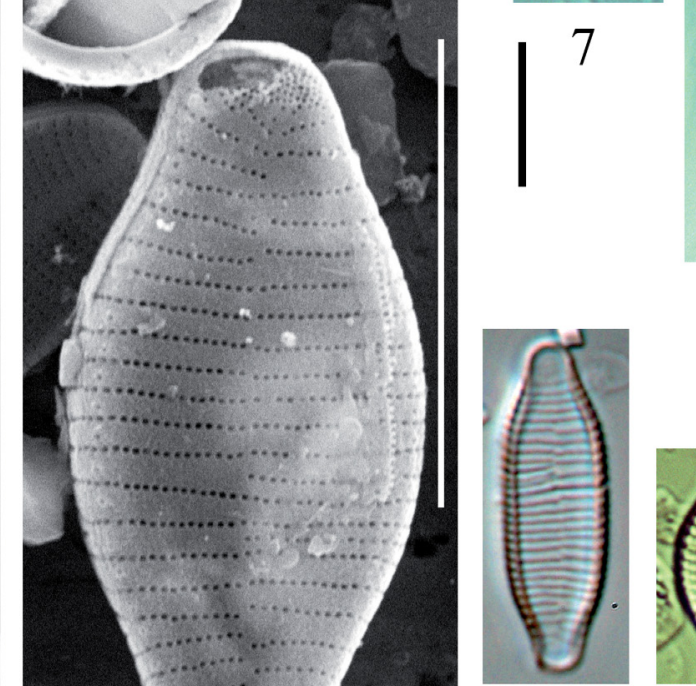

11

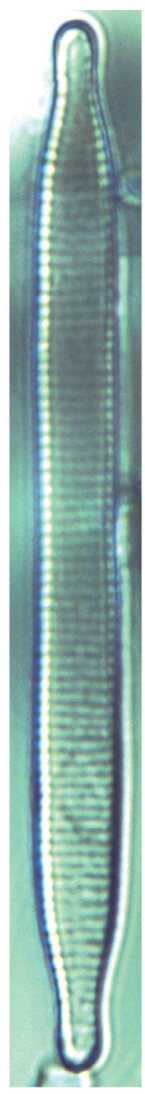

8

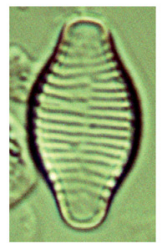

12

Plate 25: Fragilariforma virescens. - Figs 1-2: Lake Lia, LM. Fig. 3: Lake Zanoaga, LM. Fig. 4: Lake Pietrele, LM. Fig. 5: Lake Zanoaga, LM. Figs 6-8: Lake Negru, LM. Fig. 9: Lake Negru, outside view, SEM. Fig. 10: Lake Lezilor, outside view, SEM. Figs 11-12: Lake Gales, LM. Scale bars $=10 \mu \mathrm{m}$. 
Frustulia crassinervia (Brébisson ex W. Smith) Lange-Bertalot et Krammer in Lange-Bertalot et Metzeltin 1996: 57, pl. 38: figs 7-9 (Plate 26: Figs 1-7)

References: LANGe-BeRTALOT and Metzeltin (1996), LANGe-Bertalot (2001), KocioleK and GraefF (2011).

Distribution in glacial lakes in the Retezat Mountains

\begin{tabular}{ll}
\hline Lakes & Brazi, Bucura, Lia, Viorica \\
Relative abundance (max.) & $0.5 \%$ \\
Constancy & $1(16 \%)$ \\
\hline
\end{tabular}

Remark: This is a rare species in the Retezat Mts. 

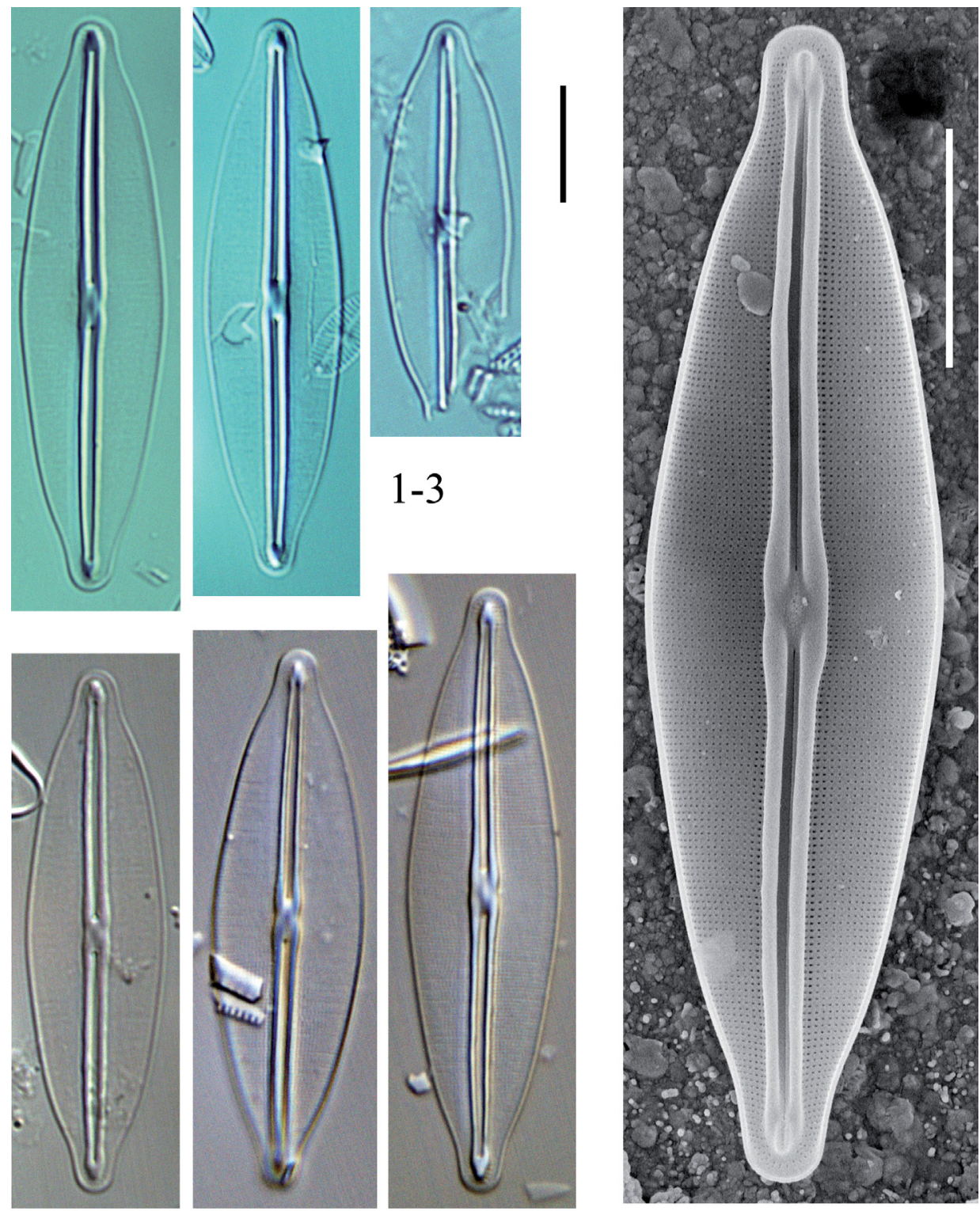

4

$5-6$

7

Plate 26: Frustulia crassinervia. - Figs 1-3: Lake Lia, LM. Fig. 4: Lake Gales, LM. Figs 5-6: Lake Bucura, LM. Fig. 7: Lake Brazi, inside view, SEM. Scale bars $=10 \mu \mathrm{m}$. 
Geissleria cf. schoenfeldii (Hustedt) Lange-Bertalot et Metzeltin 1996: 67, pl. 123: figs 5-6, pl. 124: figs 1-4

(Plate 27: Figs 1-8)

References: LANGe-BeRTAlot and Metzeltin (1996), LANGe-Bertalot (2001), HoFmANn et al. (2013).

Distribution in glacial lakes in the Retezat Mountains

\begin{tabular}{ll}
\hline Lakes & Peleguta, Lia \\
Relative abundance $(\max )$. & $1.5 \%$ \\
Constancy & $1(8 \%)$ \\
\hline
\end{tabular}

Remark: Very rare taxon in our recent study. Geissleria cf. schoenfeldii is reported from eutrophic and highly mesotrophic lakes and ponds, usually calciumcarbonate rich (LANGE-BERTALOT 2001), but not from mountain lakes. 

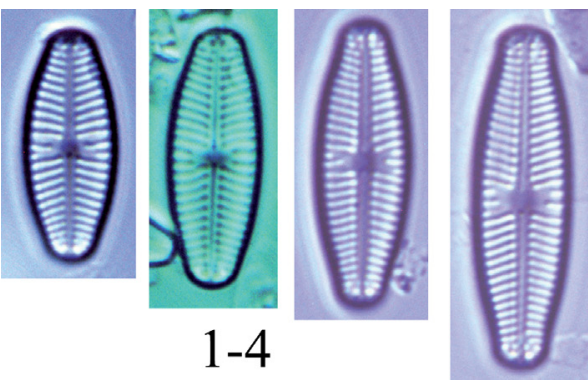

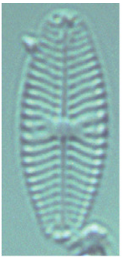

5

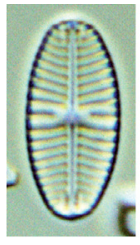

6
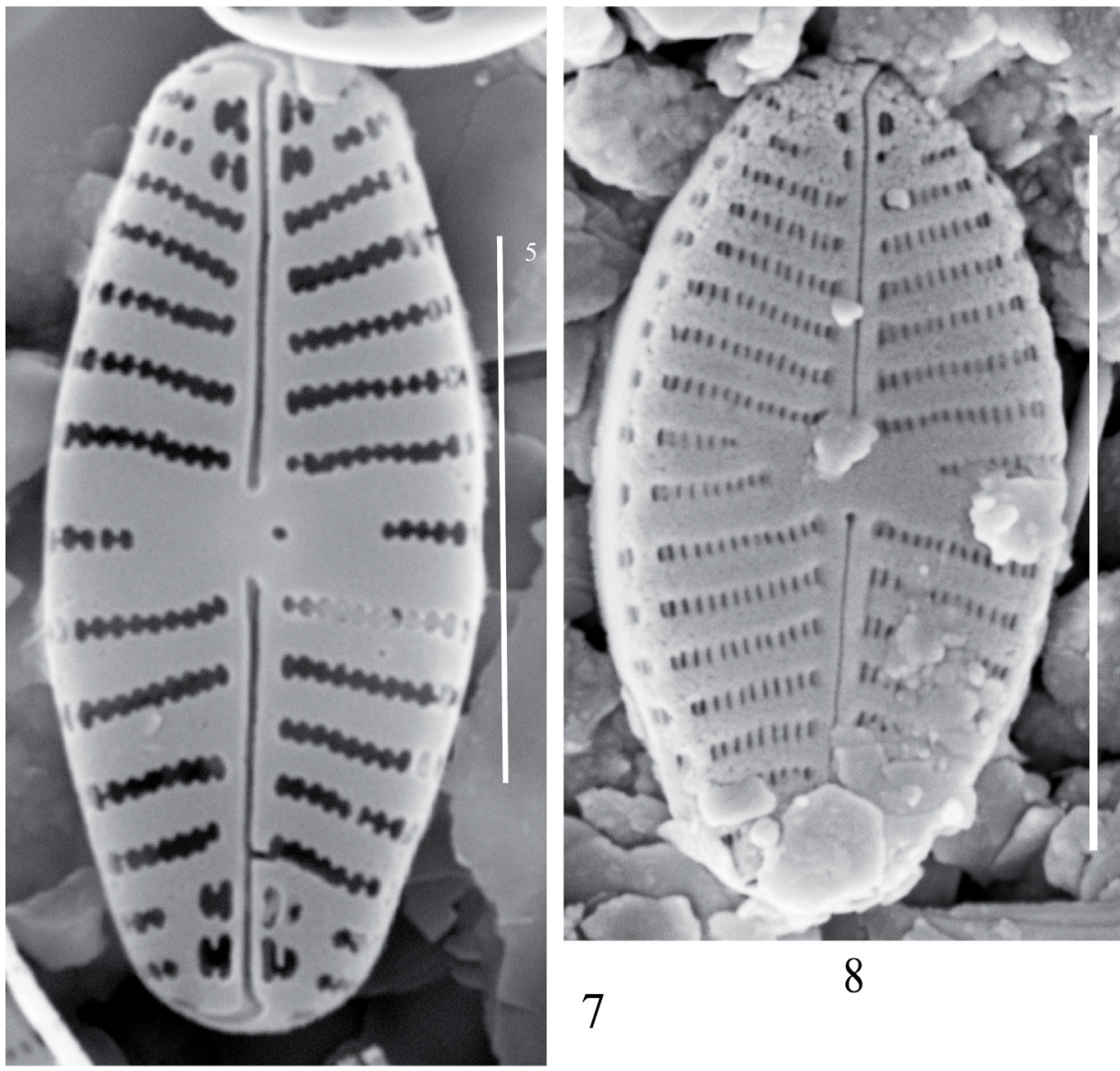

7

\section{8}

Plate 27: Geissleria cf. schoenfeldii. - Figs 1-4: Lake Peleguta, LM. Fig. 5: Lake Lia, LM. Fig. 6: Lake Peleguta. Figs 7-8: Lake Lia, outside view, SEM. Scale bars $=10 \mu \mathrm{m}$. 
Genkalia boreoalpina A. Wojtal, C. E. Wetzel, L. Ector, N.

Ognjanova-Rumenova et K. Buczkó in Wojtal, OgnjanovaRumenova, Wetzel, Hinz, Piatek, Kapetanovic, Ector et Buczkó 2014: 231, figs 49-109

(Plate 28: Figs 1-11)

Reference: Wojtal et al. (2014).

Distribution in glacial lakes in the Retezat Mountains

\begin{tabular}{ll}
\hline Lakes & $\begin{array}{l}\text { Caprelor, Gales, Peleaga, Peleguta, Pietrele, Pietrelice-2, Stirbu, } \\
\text { Viorica }\end{array}$ \\
Relative abundance $(\max )$. & $6 \%$ \\
Constancy & $2(35 \%)$ \\
\hline
\end{tabular}

Remark: This recently described species is common and frequent in the Retezat Mts. 

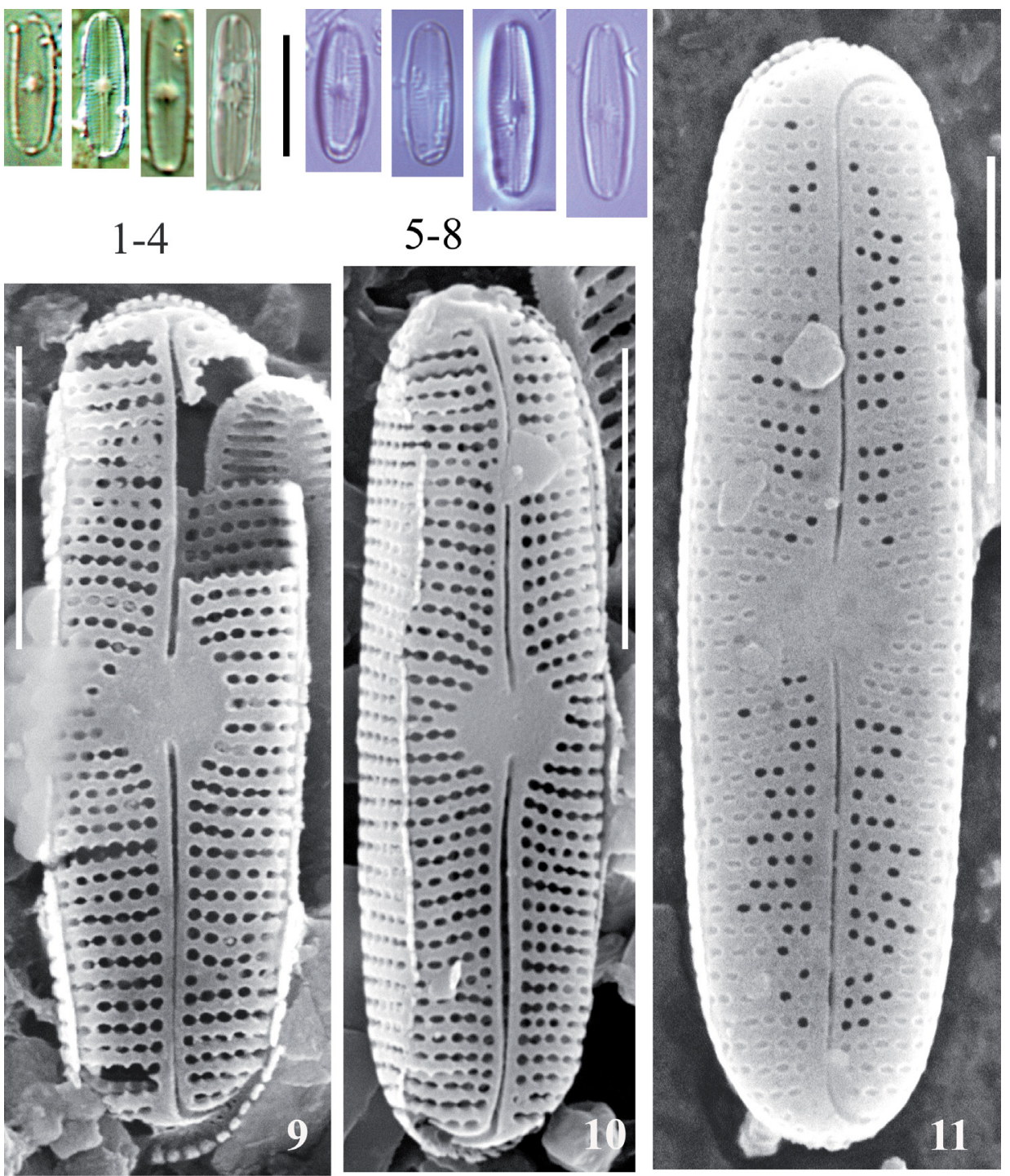

Plate 28: Genkalia boreoalpina. - Figs 1-4: Lake Brazi, LM. Figs 5-8: Lake Pietrele, LM. Figs 9-11: Lake Brazi, outside view, SEM. Scale bars $=10 \mu \mathrm{m}$. 
Genkalia digitulus (Hustedt) Lange-Bertalot et M. S. Kulikovskiy in Kulikovskiy, Lange-Bertalot, Metzeltin et Witkowski 2012: 142 (Plate 29: Figs 1-7)

References: KulikovskiY et al. (2012), HofmAnN et al. (2013), WojtAL et al. (2014).

Distribution in glacial lakes in the Retezat Mountains

\begin{tabular}{ll}
\hline Lakes & $\begin{array}{l}\text { Ana, Caprelor, Gales, Lezilor, Negru, Peleaga, Peleguta, Pietrele, } \\
\text { Pietrelice-1, Stirbu, Turcelu, Viorica }\end{array}$ \\
Relative abundance $(\max )$. & $5 \%$ \\
Constancy & $3(48 \%)$ \\
\hline
\end{tabular}

Remarks: PÉTERFi (1993) reported this species from lakes as Navicula digitulus. Common and fairly frequent in the lakes of the Retezat Mts. 

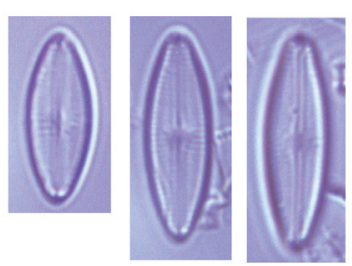

$1-5$
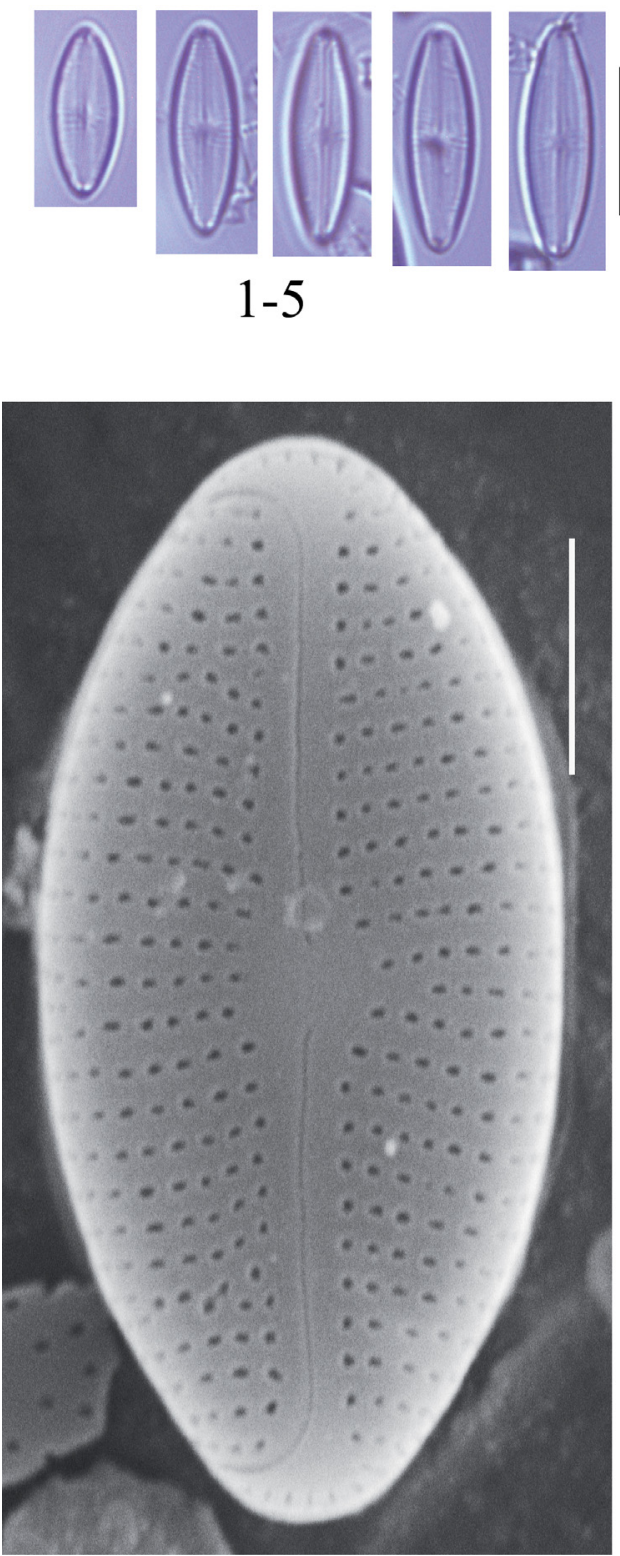

6

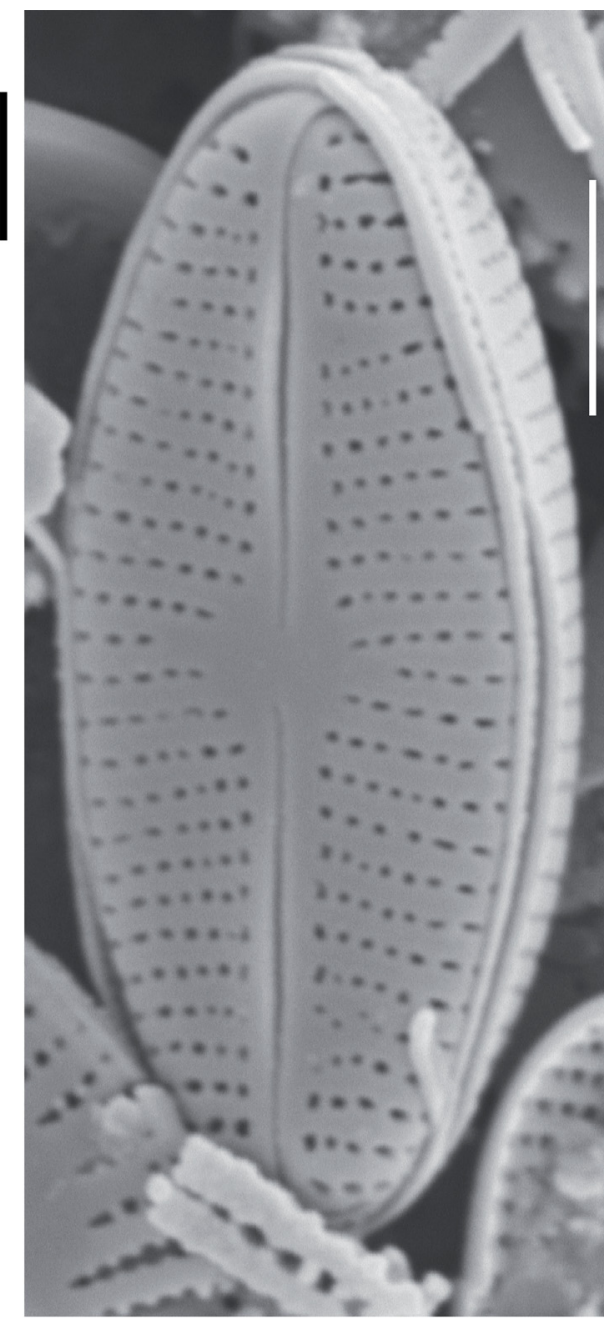

7

Plate 29: Genkalia digitulus. - Figs 1-5: Lake Pietrele, LM. Fig 6: Lake Peleguta, outside view, SEM. Fig. 7: Lake Pietrele, outside view, SEM. Scale bars $=10 \mu \mathrm{m}$. 
Genkalia subprocera (Hustedt) A. Wojtal, L. Ector, C. E. Wetzel, N. Ognjanova-Rumenova et K. Buczkó in Wojtal, OgnjanovaRumenova, Wetzel, Hinz, Piatek, Kapetanovic, Ector et Buczkó 2014: 232

(Plate 30: Figs 1-9)

Reference: Wojtal et al. (2014).

Distribution in glacial lakes in the Retezat Mountains

\begin{tabular}{ll}
\hline Lakes & Caprelor, Lia, Pietrele \\
Relative abundance $(\max )$. & $0.5 \%$ \\
Constancy & $1(12 \%)$ \\
\hline
\end{tabular}

Remark: This is a rare and never abundant taxon in our recent study. 

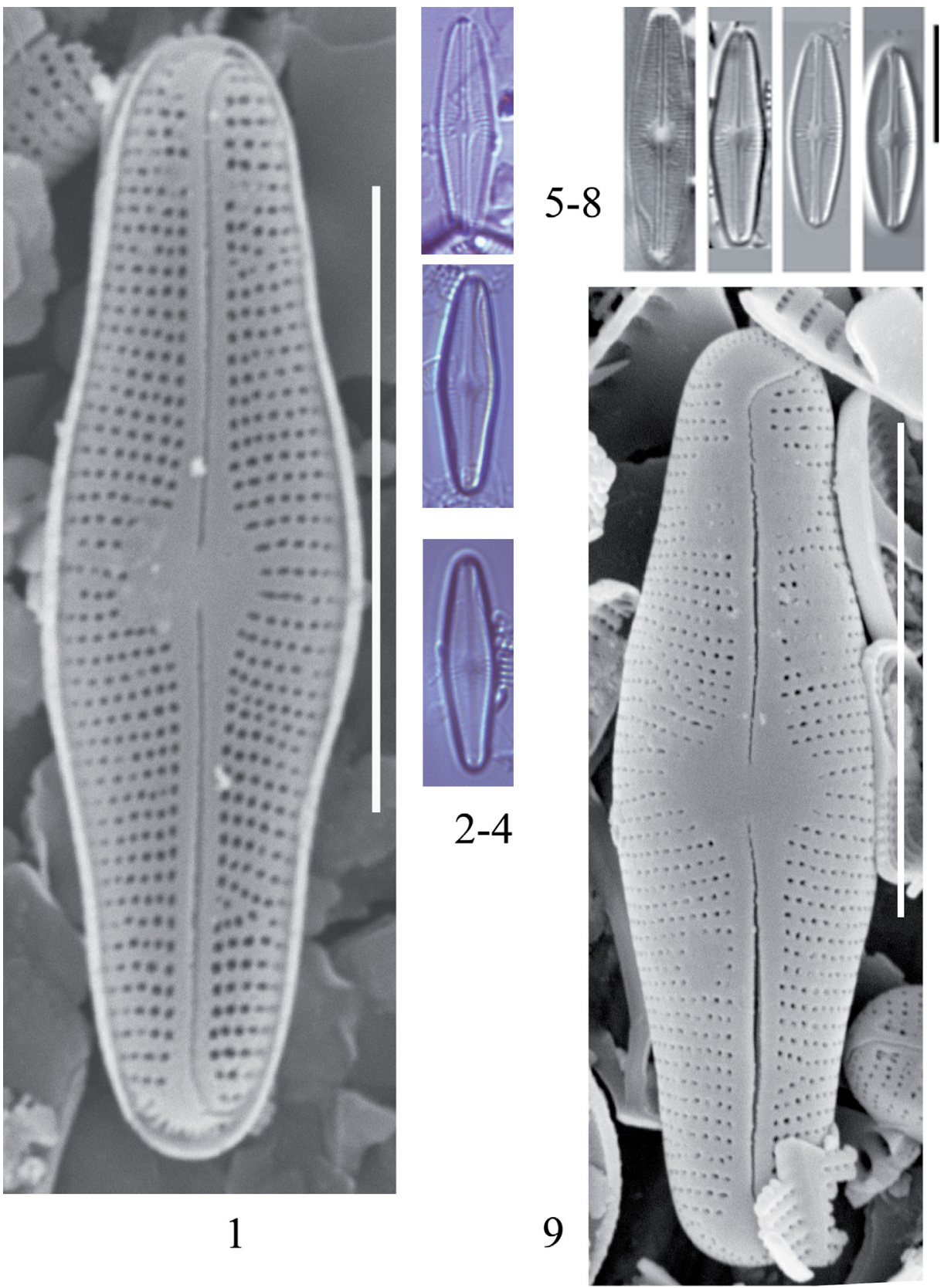

Plate 30: Genkalia subprocera. - Fig. 1: Lake Lia, outside view, SEM. Figs 2-8: Lake Pietrele, LM. Fig. 9: Lake Pietrele, outside view, SEM. Scale bars $=10 \mu \mathrm{m}$. 
Hannaea arcus (Ehrenberg) R. M. Patrick in R. M. Patrick et L. R. Freese 1961: 132, pl. 4: fig. 20

(Plate 31: Figs 1-8)

References: Patrick and Freese (1961), Kociolek (2010), HofmanN et al. (2013).

Distribution in glacial lakes in the Retezat Mountains

\begin{tabular}{ll}
\hline Lakes & Lia, Gales \\
Relative abundance (max.) & $0.5 \%$ \\
Constancy & $1(8 \%)$ \\
\hline
\end{tabular}

Remarks: PÉTERFI (1993) reported this species from lakes and running waters. $H$. arcus var. amphioxys was also mentioned in this publication. Rare species. 

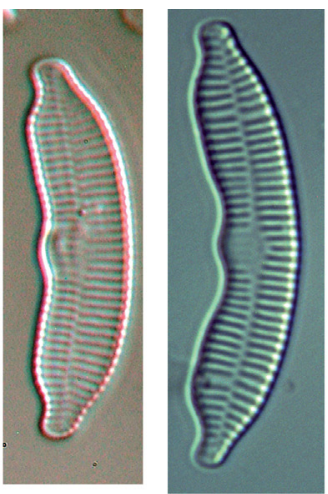

1

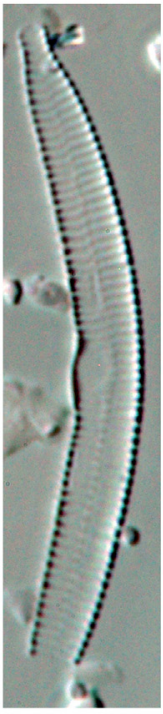

4

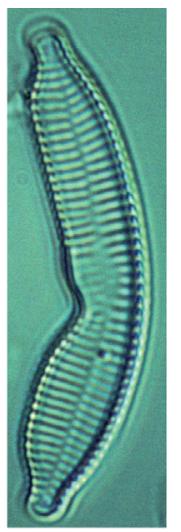

3

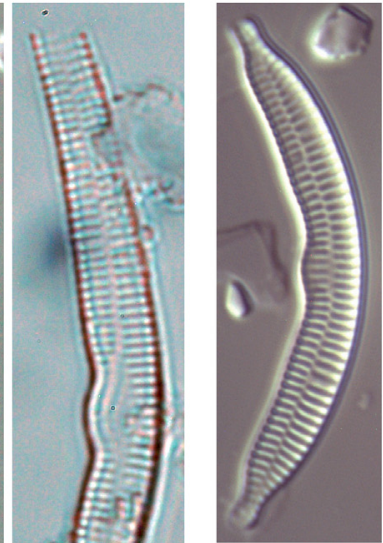

6

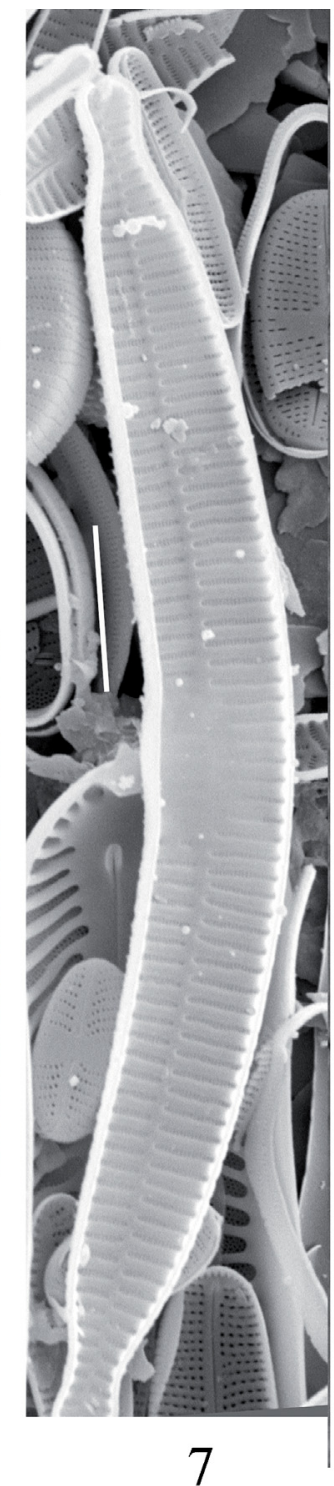

7

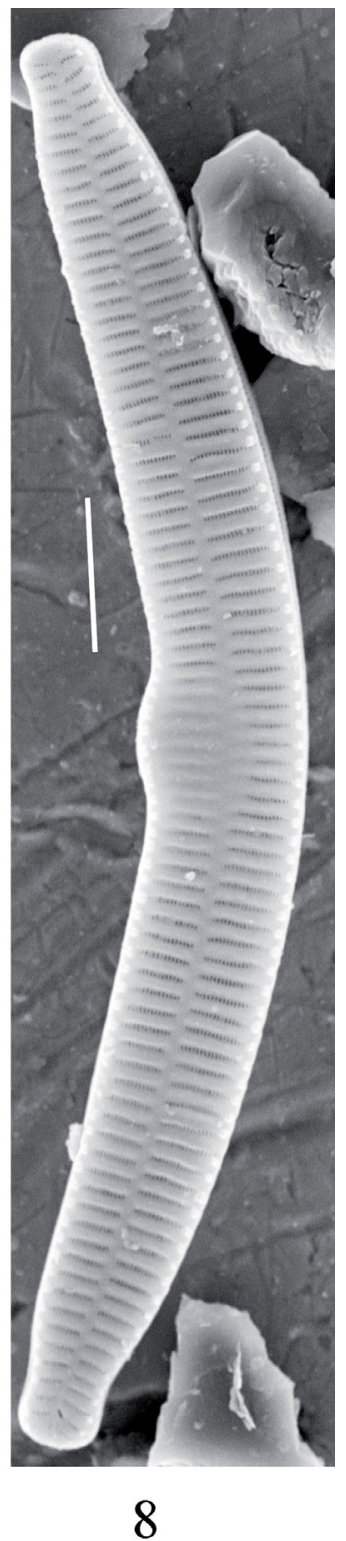

Plate 31: Hannaea arcus. - Fig. 1: Lake Gales, LM. Figs 2-3: Lake Lia, LM. Figs 4-5: Lake Gales, LM. Fig. 6: Lake Lia, LM. Fig. 7: Lake Gales, inside view, SEM. Fig. 8: Lake Lia, outside view, SEM. Scale bars $=10 \mu \mathrm{m}$. 
Humidophila fukushimae (Lange-Bertalot, M. Werum et A. Broszinski in Lange-Bertalot et Werum) Buczkó et Kövér 2015 (Plate 32: Figs 1-13)

References: LANGE-BERTALOT and WeRUM (2001), WERUM and LANGEBertalot (2004), Lowe et al. (2014), KÖvÉr et al. (2015).

Distribution in glacial lakes in the Retezat Mountains

\begin{tabular}{ll}
\hline Lakes & Brazi, Negru \\
Relative abundance $(\max )$. & $0.5 \%$ \\
Constancy & $1(8 \%)$ \\
\hline
\end{tabular}

Remark: This is a very rare species in the Retezat Mts, as well as in Europe. 

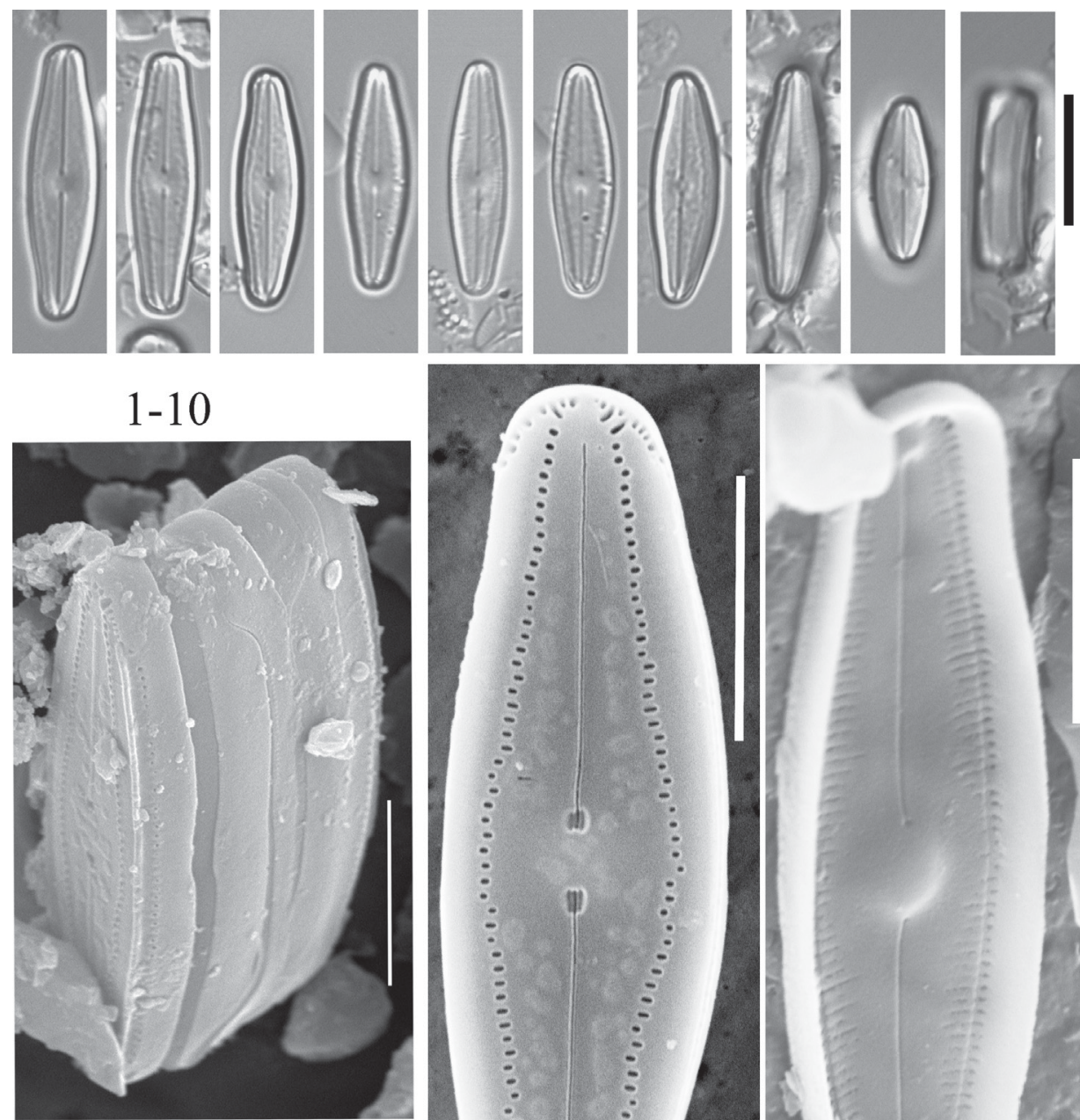

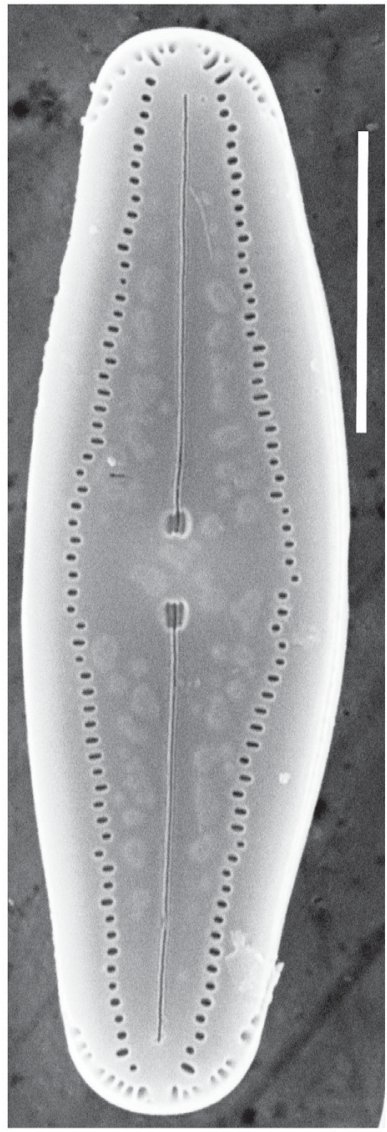

12

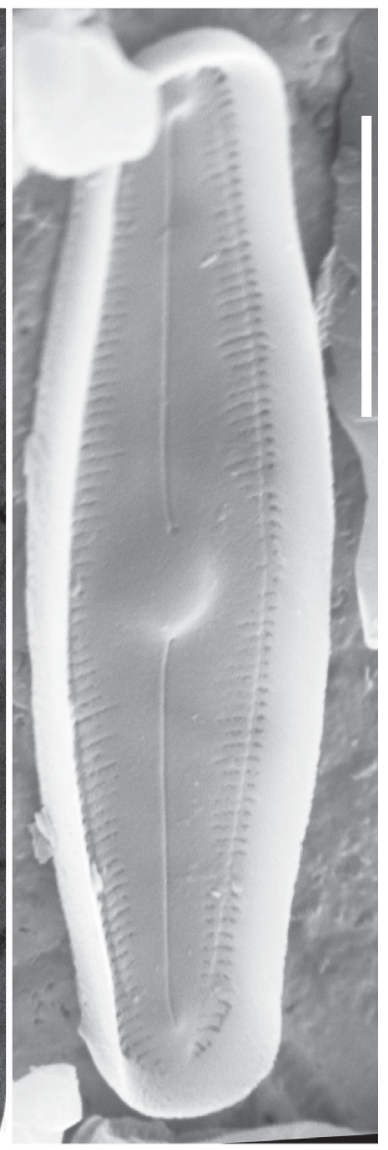

11

13

Plate 32: Humidophila fukushimae. - Figs 1-10: Lake Negru, LM. Fig. 11: Lake Negru, girdle view, SEM. Fig. 12: Lake Negru, outside view, SEM. Fig. 13: Lake Negru, inside view, SEM. Scale bars $=10 \mu \mathrm{m}$. 


\section{Humidophila sp.}

(Plate 33: Figs 1-14)

Distribution in glacial lakes in the Retezat Mountains

Lakes

Caprelor, Gales, Gemenele, Lezilor, Lia, Negru, Peleaga, Peleguta, Stirbu, Viorica, Zanoaga

Relative abundance $(\max ) \quad 2 \$.

Constancy $3(48 \%)$

Remarks: We suppose this is a new species. Valves are linear-lanceolate to lanceolate, 4.0-13.4 $\mu \mathrm{m}$ (average 8.3 \pm 1.7 ) long, 2.0-2.5 $\mu \mathrm{m}$ (average 2.2 $\pm 0.4 ; \mathrm{n}=$ 31) wide. Ends are rostrate-subcapitate. Raphe (filiform, straight) simple, threadlike with simple terminals, the proximal terminals are well visible. Axial area is hardly visible, central area is small. Striae are rarely discernible. Common and sometimes abundant species in the Retezat Mts. 

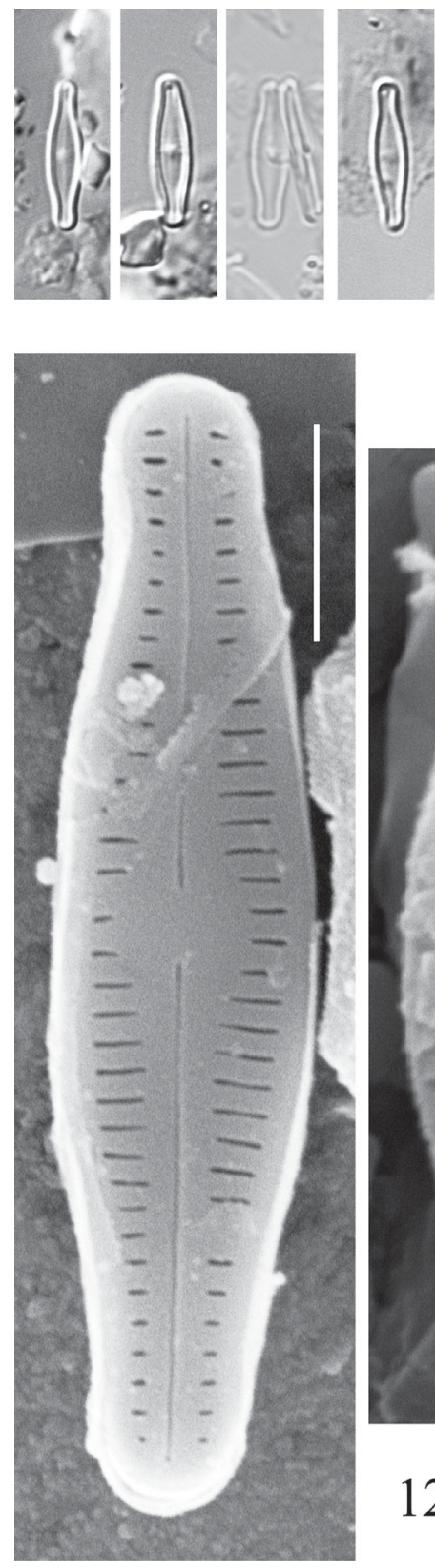
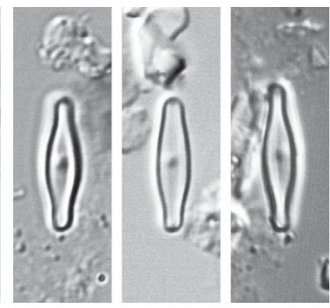

$1-10$

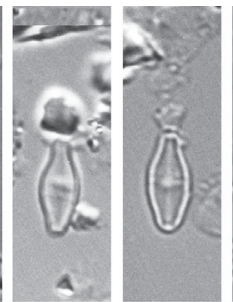

4

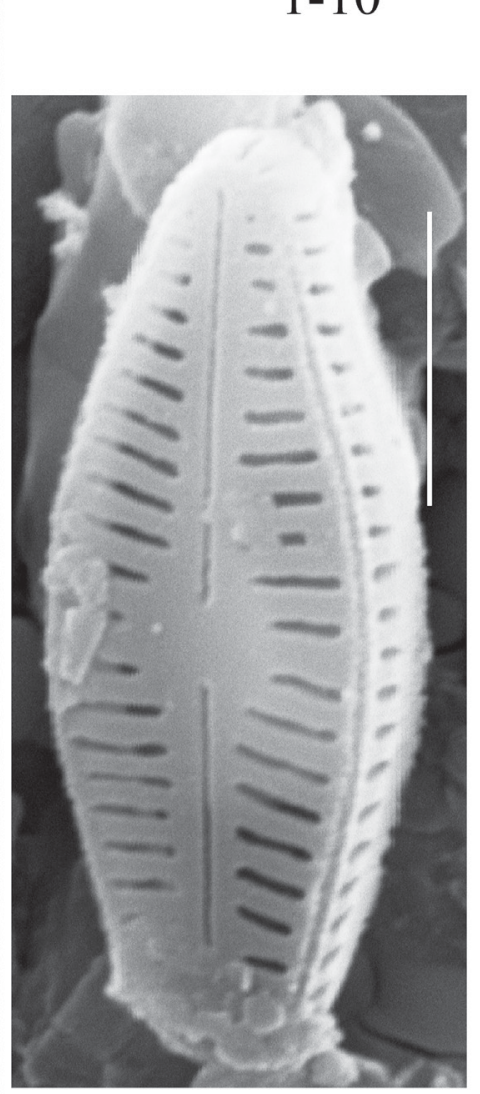

13

12
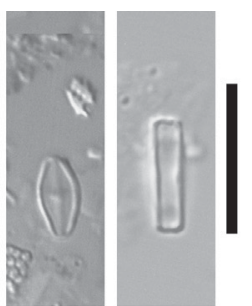

11

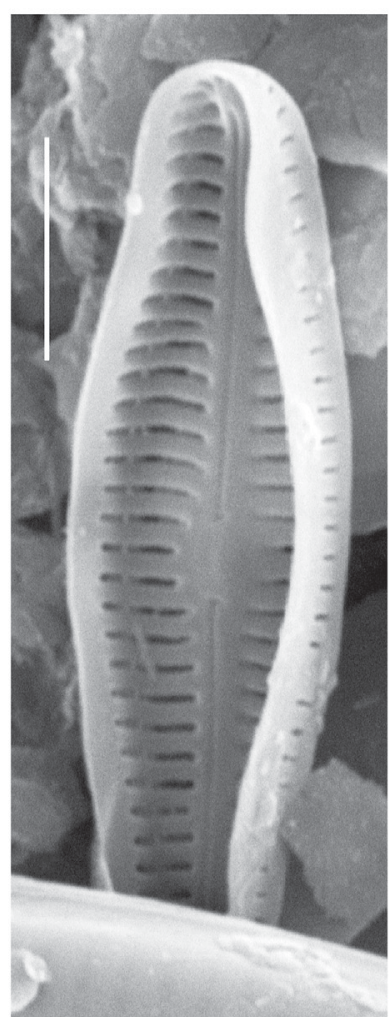

14

Plate 33: Humidophila sp. - Figs 1-10: Lake Gales, LM. Fig. 11: Lake Gales, girdle view, LM. Fig. 12: Lake Gales, outside view, SEM. Fig. 13: Lake Gales, outside view, oblique position, SEM. Fig. 14: Lake Gales, inside view, SEM. Scale bar $=10 \mu \mathrm{m}$ (Figs 1-10), $2 \mu \mathrm{m}$ (Figs 12-14). 
Humidophila schmassmannii (Hustedt) K. Buczkó et A. Wojtal in Buczkó, Wojtal, Beszteri et Magyari 2015: 31

(Plate 34: Figs 1-29)

References: Hustedt (1943, 1962), WERUM and LANGE-BERTALOT (2004), OTu and SPAulding (2011b), Buczkó et al. (2015).

Distribution in glacial lakes in the Retezat Mountains

\begin{tabular}{ll}
\hline Lakes & Caprelor, Gales, Lezilor, Peleaga, Peleguta, Stirbu \\
Relative abundance $(\max )$. & $24 \%$ \\
Constancy & $2(28 \%)$ \\
\hline
\end{tabular}

Remark: Not rare, sometimes quite abundant species in the mountain lakes of the Retezat Mts. 

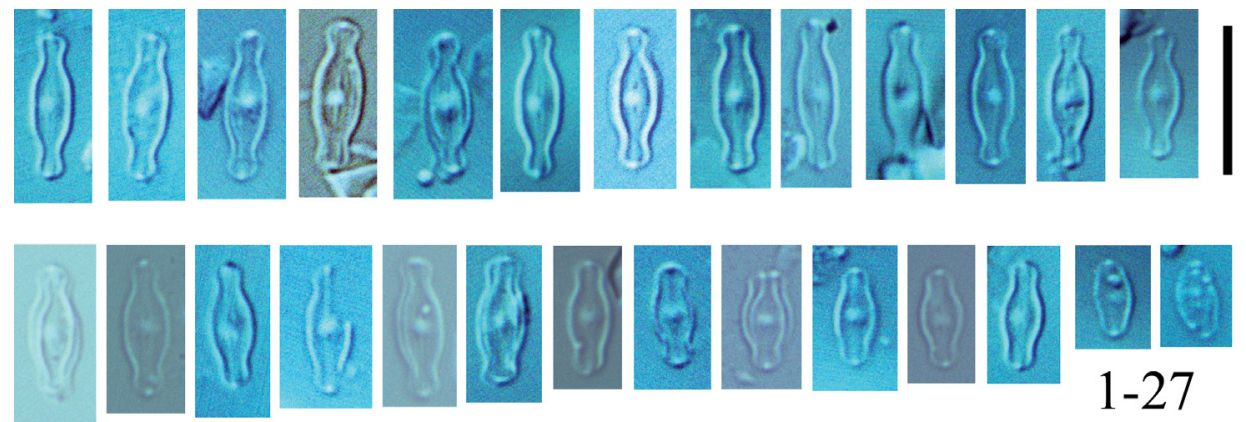

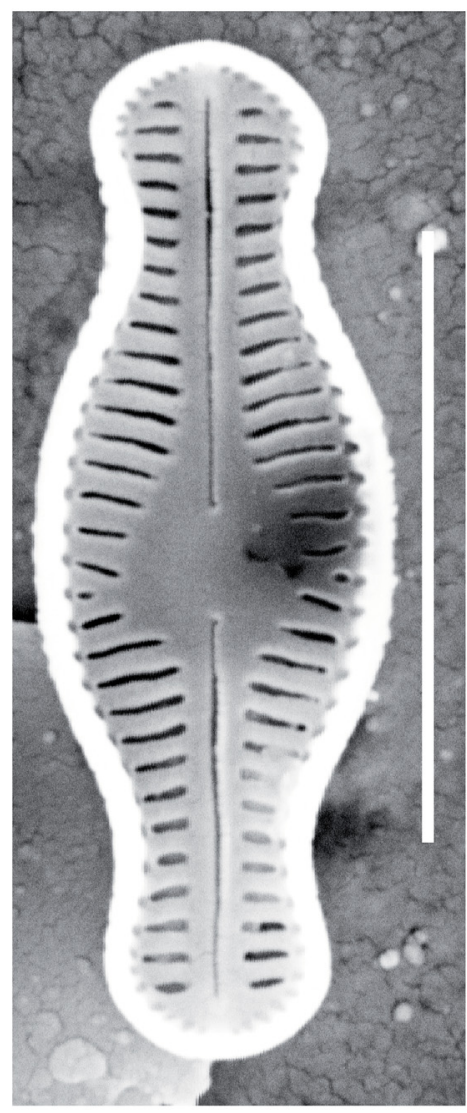

28

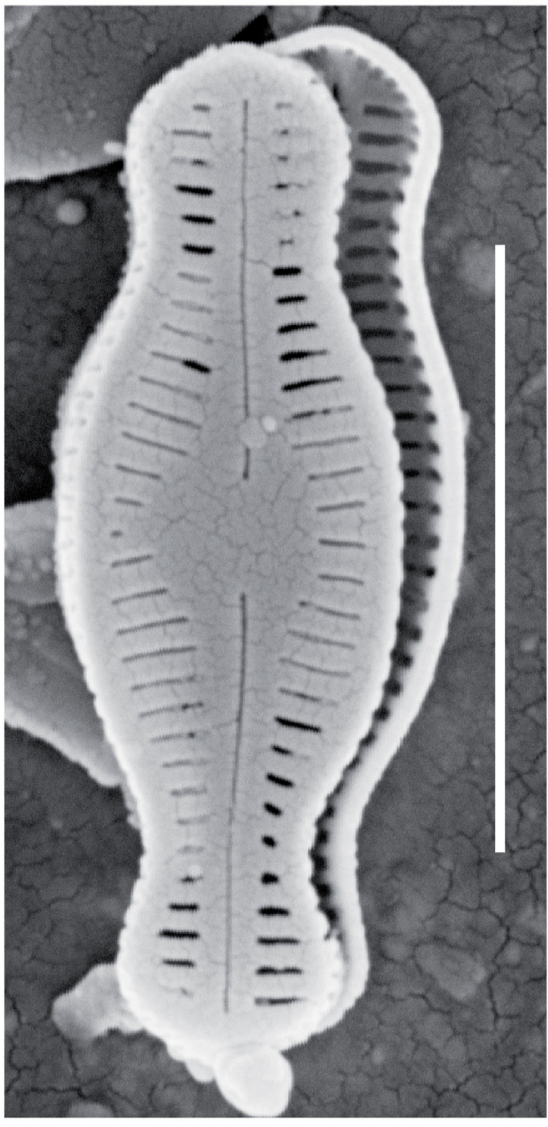

29

Plate 34: Humidophila schmassmannii. - Figs 1-27: Lake Lia, LM. Fig. 28: Lake Lia, inside view, SEM. Fig. 29: Lake Lia, outside view, SEM. Scale bars $=10 \mu \mathrm{m}$ (Figs 1-27), $5 \mu \mathrm{m}$ (Figs 28-29). 


\section{Meridion circulare (Greville) C. Agardh 1831: 40}

(Plate 35: Figs 1-10)

References: AgARdH (1831), Kociolex (2011a), HofmAnN et al. (2013).

Distribution in glacial lakes in the Retezat Mountains

\begin{tabular}{ll}
\hline Lakes & Lia \\
Relative abundance $(\max )$. & $0.5 \%$ \\
Constancy & $1(4 \%)$ \\
\hline
\end{tabular}

Remarks: PÉTERFI (1993) reported this species from mires and running waters. In our recent study we found only in one lake. Rare. 

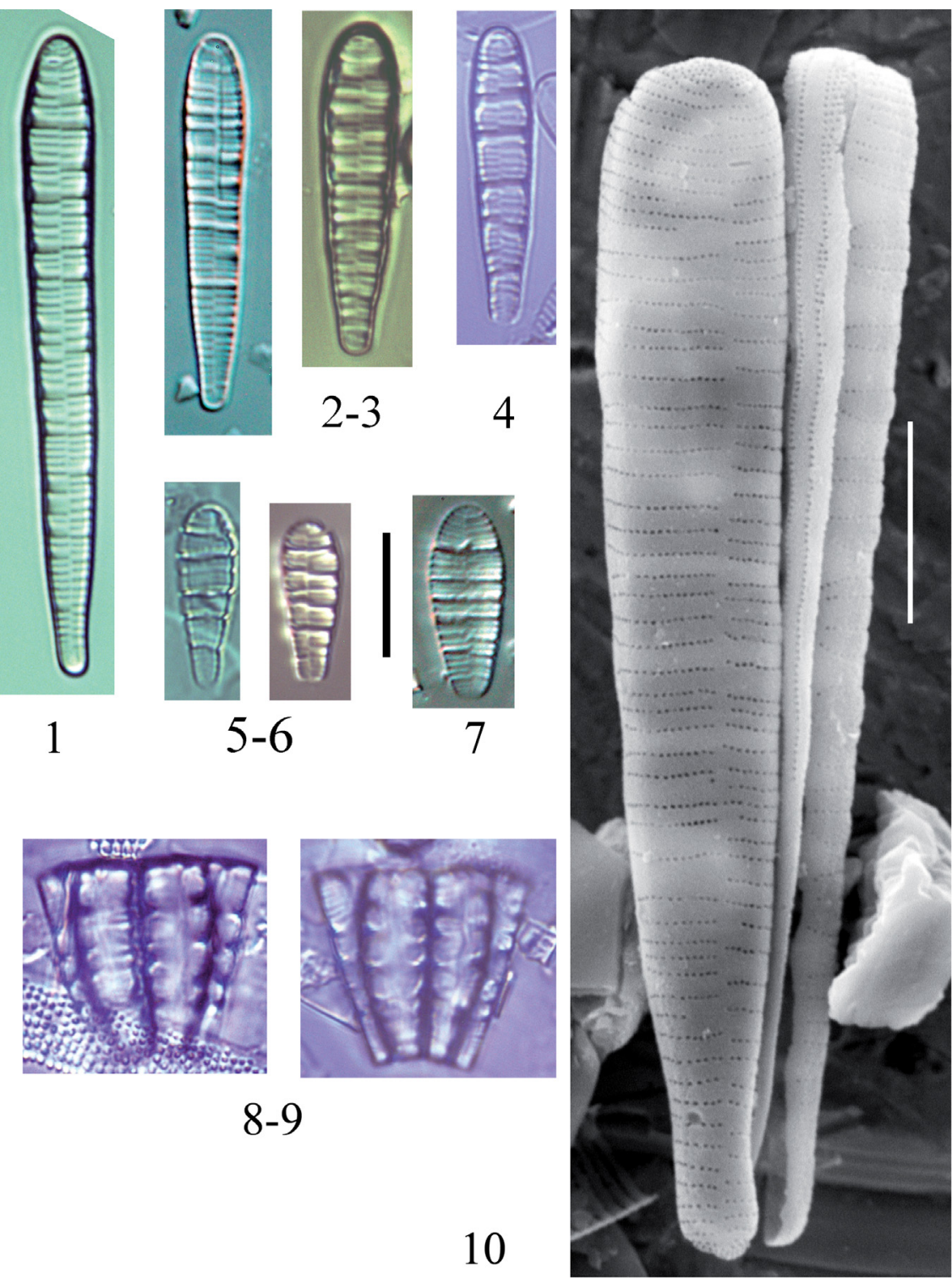

10
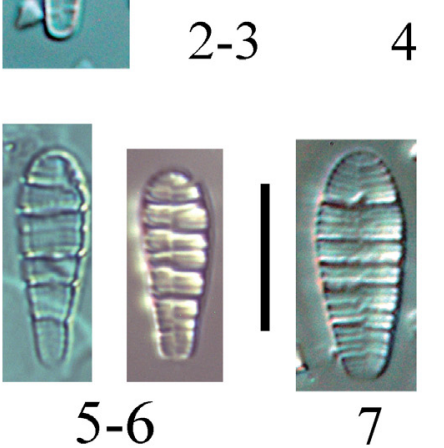

4

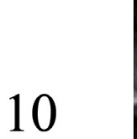

Plate 35: Meridion circulare. - Fig. 1: Lake Lia, LM. Figs 2-3: Lake Gales, LM. Fig. 4: Lake Pietrele, LM. Figs 5-6: Lake Lia, LM. Fig. 7: Lake Gales, LM. Figs 8-9: Lake Zanoaga, LM. Fig. 10: Lake Lia, SEM outside view. Scale bars $=10 \mu \mathrm{m}$. 
Meridion circulare var. constrictum (Ralfs) Van Heurck 1880: pl. 51: figs 14-15

(Plate 36: Figs 1-8)

References: VAN Heurck (1880), Hofmann et al. (2013), Hoidal (2013).

Distribution in glacial lakes in the Retezat Mountains

\begin{tabular}{ll}
\hline Lakes & Lia, Gales, Iezior, Negru, Stavieu, Zanoaga \\
Relative abundance (max.) & $0.5 \%$ \\
Constancy & $2(21 \%)$ \\
\hline
\end{tabular}

Remarks: PÉTERFI (1993) reported this species from lakes and running waters. Not rare in our recent study. 


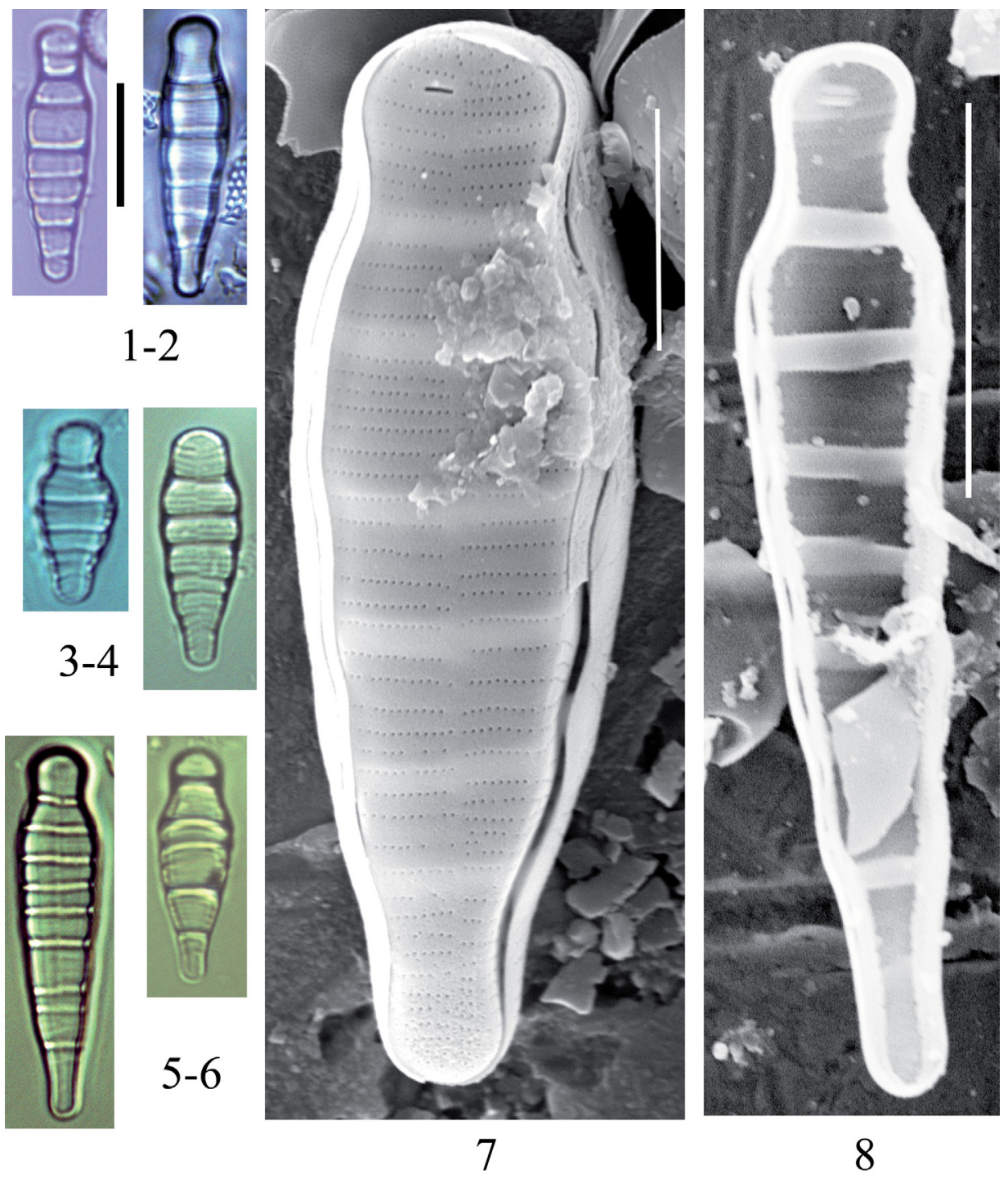

Plate 36: Meridion circulare var. constrictum. - Figs 1-2: Lake Zanoaga, LM. Figs 3-4: Lake Lia, LM. Figs 5-6: Lake Gales, LM. Fig. 7: Lake Lia, outside view, SEM. Fig. 8: Lake Zanoaga, inside view, SEM. Scale bars $=10 \mu \mathrm{m}$. 
Microcostatus krasskei (Hustedt) Johansen et Sray 1998: 98 (Plate 37: Figs 1-10)

References: Johansen and SRAY (1998), Hofmann et al. (2013), Lowe (2015).

Distribution in glacial lakes in the Retezat Mountains

\begin{tabular}{ll}
\hline Lakes & Gales, Lia, Negru, Pietrelice \\
Relative abundance (max.) & $0.5 \%$ \\
Constancy & $1(12 \%)$ \\
\hline
\end{tabular}

Remark: Never abundant, but not too rare species in our recent study. 

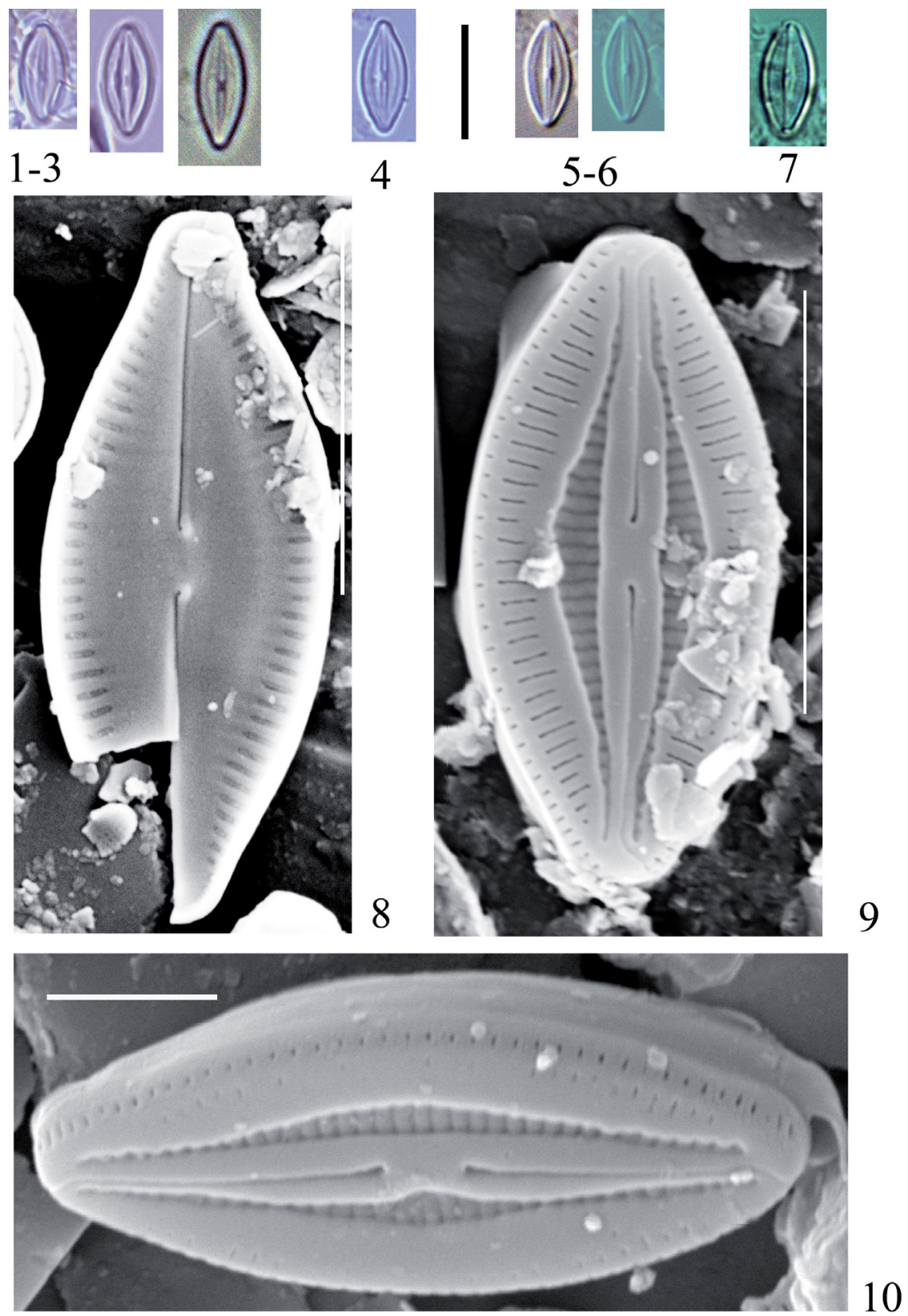

Plate 37: Microcostatus krasskei. - Figs 1-3: Lake Negru, LM. Fig 4: Lake Pietrelice, LM. Figs 5-6: Lake Gales, LM. Fig. 7: Lake Lia, LM. Fig. 8: Lake Negru, inside view, SEM. Fig. 9: Lake Lia, outside view, SEM. Fig. 10: Lake Negru, outside view, oblique position, SEM. Scale bars $=10 \mu \mathrm{m}$ (Figs 1-9), $2 \mu \mathrm{m}$ (Fig. 10). 
Microfissurata paludosa Cantonati et Lange-Bertalot in Cantonati, Van de Vijver et Lange-Bertalot 2009: 735-736, fig. 1 (Plate 38: Figs 1-4)

Reference: Cantonati et al. (2009).

Distribution in glacial lakes in the Retezat Mountains

\begin{tabular}{ll}
\hline Lakes & Brazi, Negru, Pietrele \\
Relative abundance (max.) & $0.5 \%$ \\
Constancy & $1(12 \%)$ \\
\hline
\end{tabular}

Remark: Rare species. 

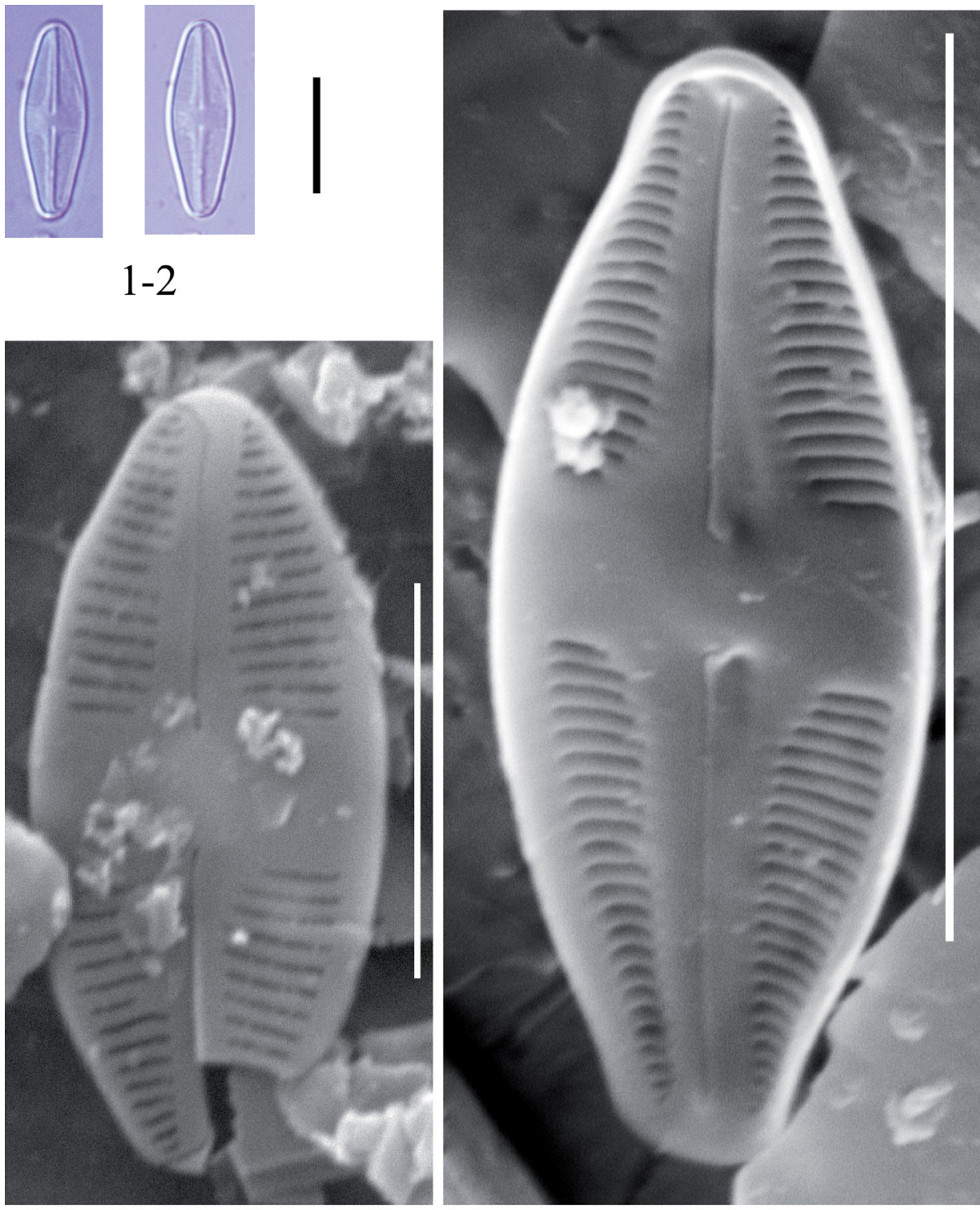

3

4

Plate 38: Microfissurata paludosa. - Figs 1-2: Lake Pietrele, LM. Fig 3: Lake Brazi, inside view, SEM. Fig. 4: Lake Negru, inside view, SEM. Scale bars $=10 \mu \mathrm{m}$. 
Navicula cryptocephala Kützing 1844: 95, pl. 3: figs 20, 26

(Plate 39: Figs 1-13)

References: KÜtZING (1844), KRAMMER and LANGE-BERTALOT (1991), Potapova (2011a), Hofmann et al. (2013).

Distribution in glacial lakes in the Retezat Mountains

\begin{tabular}{ll}
\hline Lakes & Peleguta \\
Relative abundance $(\max )$. & $0.5 \%$ \\
Constancy & $1(4 \%)$ \\
\hline
\end{tabular}

Remarks: PÉTERFI (1993) reported this species from running waters. This is a rare species in our recent study. 

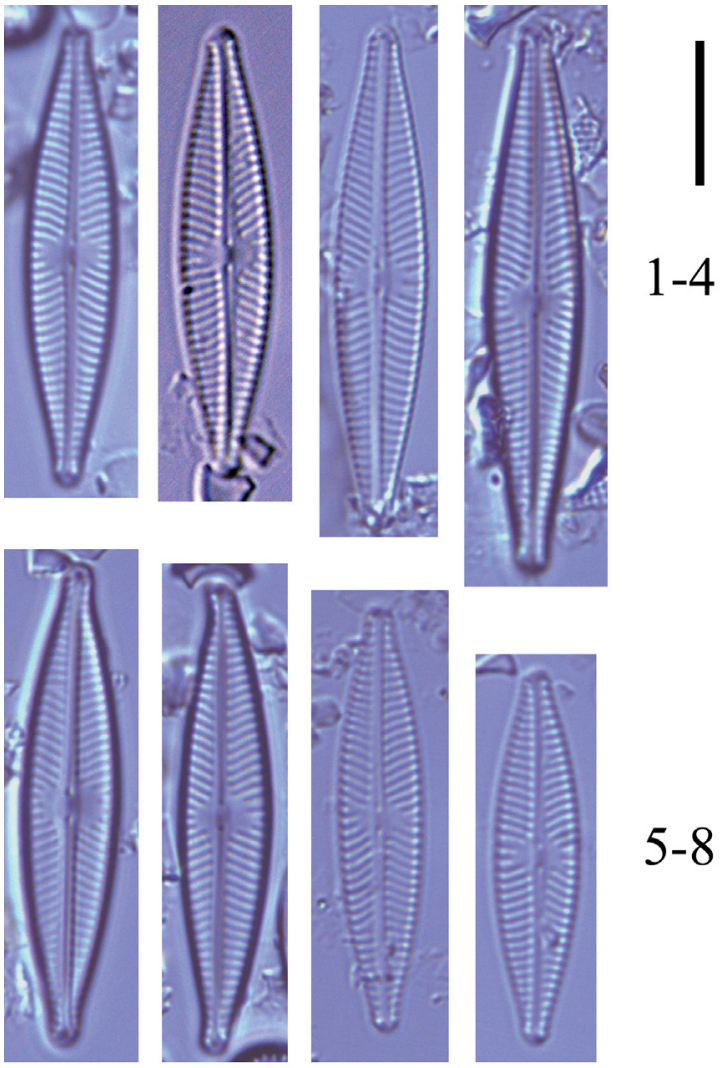

5-8
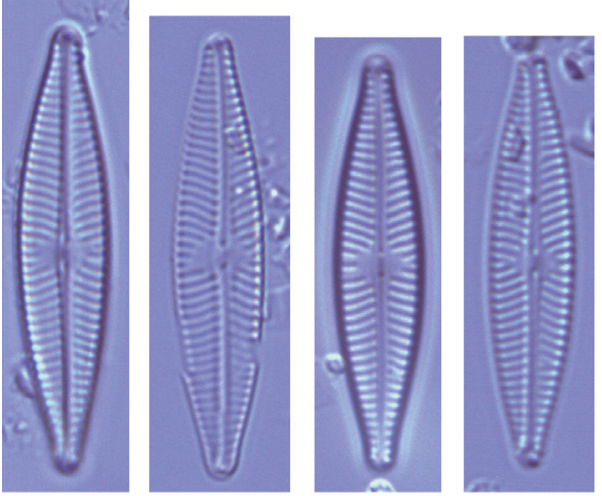

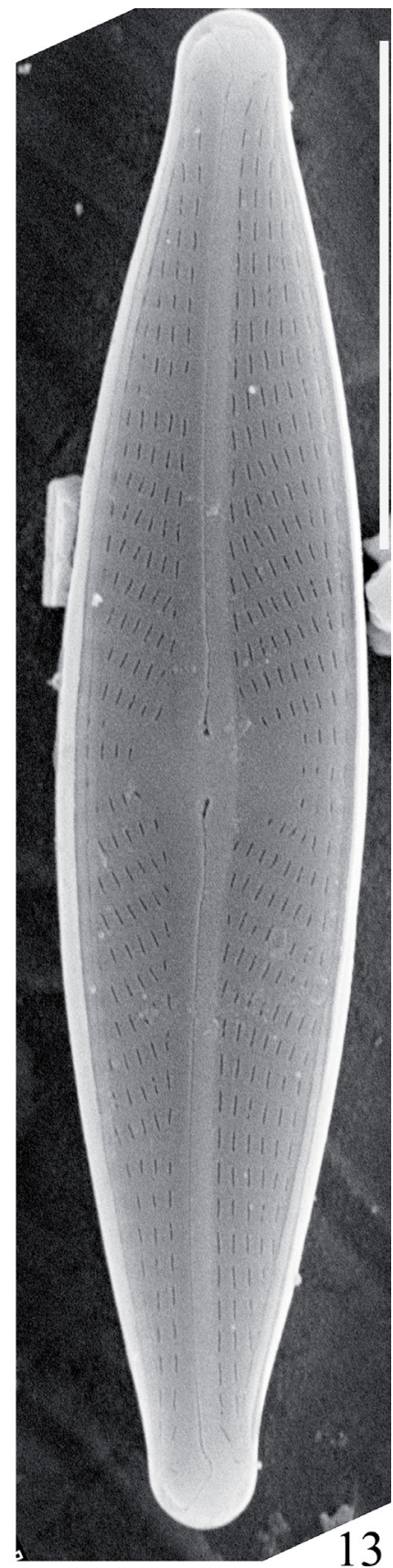

13

Plate 39: Navicula cryptocephala. - Figs 1-12: Lake Peleguta, LM. Fig. 13: Lake Peleguta, outside view, SEM. Scale bars $=10 \mu \mathrm{m}$. 
Navicula rhynchocephala Kützing 1844: 152, pl. 30: fig. 35 (Plate 40: Figs 1-9)

References: KÜtZING (1844), Krammer and LANGe-Bertalot (1991), Kociolek (2011b), Hofmann et al. (2013).

Distribution in glacial lakes in the Retezat Mountains

\begin{tabular}{ll}
\hline Lakes & Gales, Peleguta \\
Relative abundance $(\max )$. & $0.5 \%$ \\
Constancy & $1(8 \%)$ \\
\hline
\end{tabular}

Remarks: PÉTERFI (1993) reported this species from running waters. Quite rare species in the Retezat Mts. 

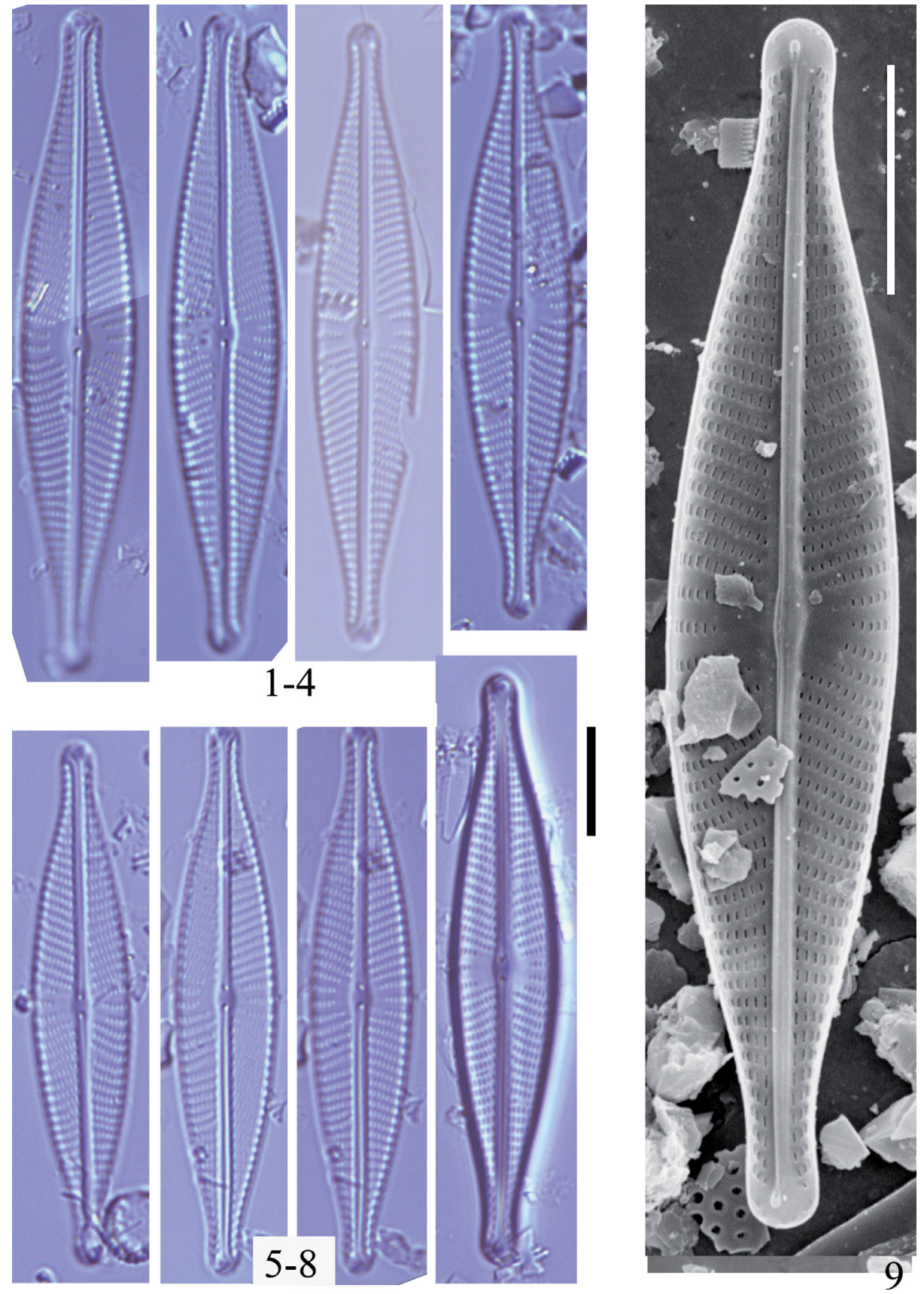

Plate 40: Navicula rhynchocephala. - Figs 1-8: Lake Pelaguta, LM. Fig. 9: Lake Pelaguta outside view, SEM. Scale bars $=10 \mu \mathrm{m}$. 
Neidium affine var. longiceps (W. Gregory) Cleve 1894: 68

(Plate 41: Figs 1-10)

References: Cleve (1894), Hofmann et al. (2013) as Neidium longiceps.

Distribution in glacial lakes in the Retezat Mountains

Lakes

Brazi, Lia, Zanoaga

Relative abundance ( $\max ) \quad 0.5 \$.

Constancy $1(8 \%)$

Remarks: PÉTERFI (1993) reported this variety from mires and running waters. "Neidium affine et vars." was also published from glacial lakes (PÉTERFI 1993). Rare species in our recent study. 

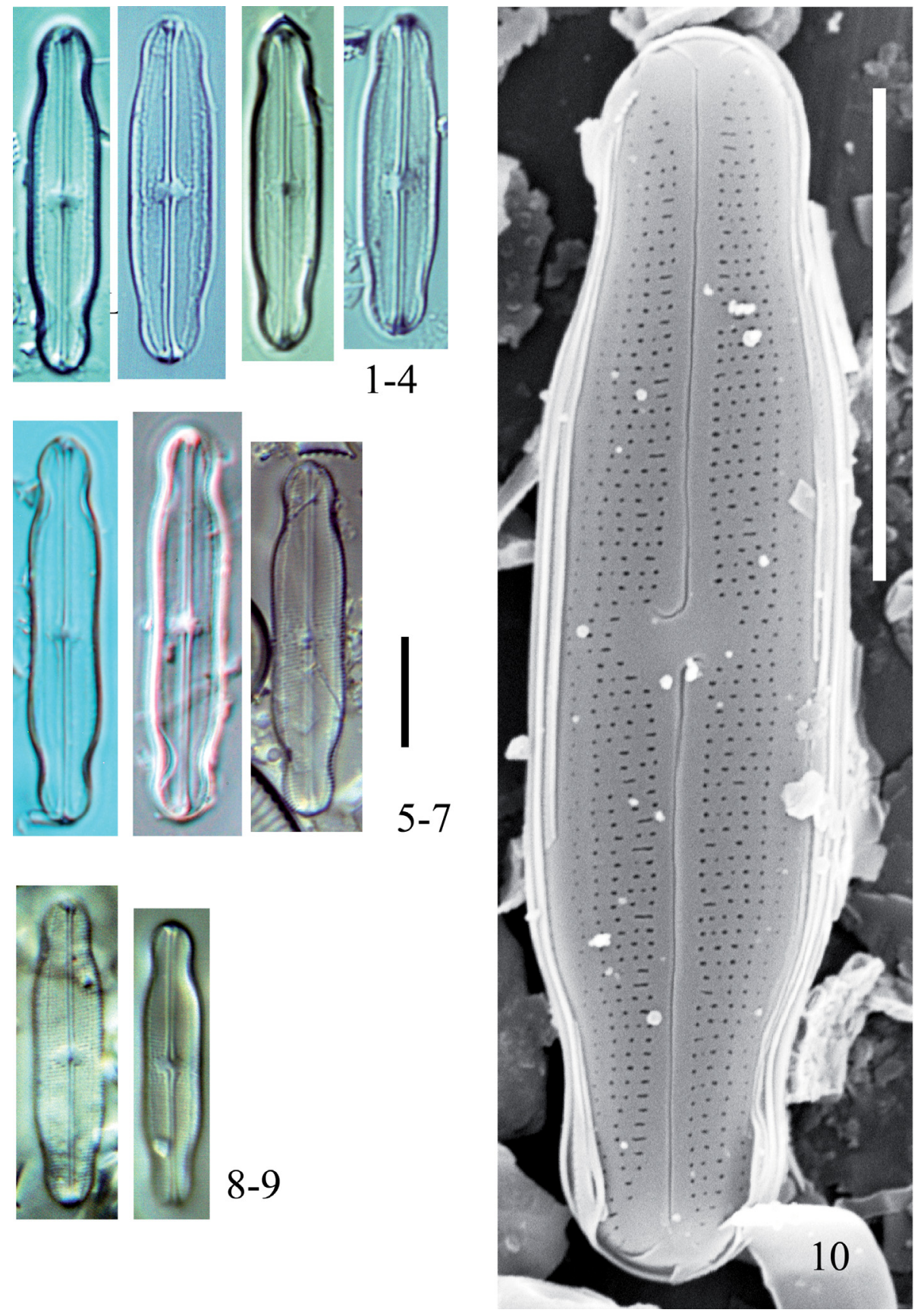

Plate 41: Neidium affine var. longiceps. - Figs 1-4: Lake Gales, LM. Figs 5-7: Lake Brazi, LM. Figs 8-9: Lake Zanoaga, LM. Fig. 10: Lake Lia, outside view, SEM. Scale bars $=10 \mu \mathrm{m}$. 
Neidium alpinum Hustedt 1943: 139, fig. 48 (Plate 42: Figs 1-16)

References: Hustedt (1943), Krammer and LANge-Bertalot (1991), HOFMANN et al. (2013).

Distribution in glacial lakes in the Retezat Mountains

\begin{tabular}{ll}
\hline Lakes & $\begin{array}{l}\text { Caprelor, Florica, Gemenele, Lezilor, Lia, Peleaga, Peleguta, Ste- } \\
\text { via, Stirbu, Turcelu, Viorica, Zanoaga }\end{array}$ \\
Relative abundance (max.) & $3.5 \%$ \\
Constancy & $3(52 \%)$ \\
\hline
\end{tabular}

Remark: Common and sometimes abundant species is this study. 


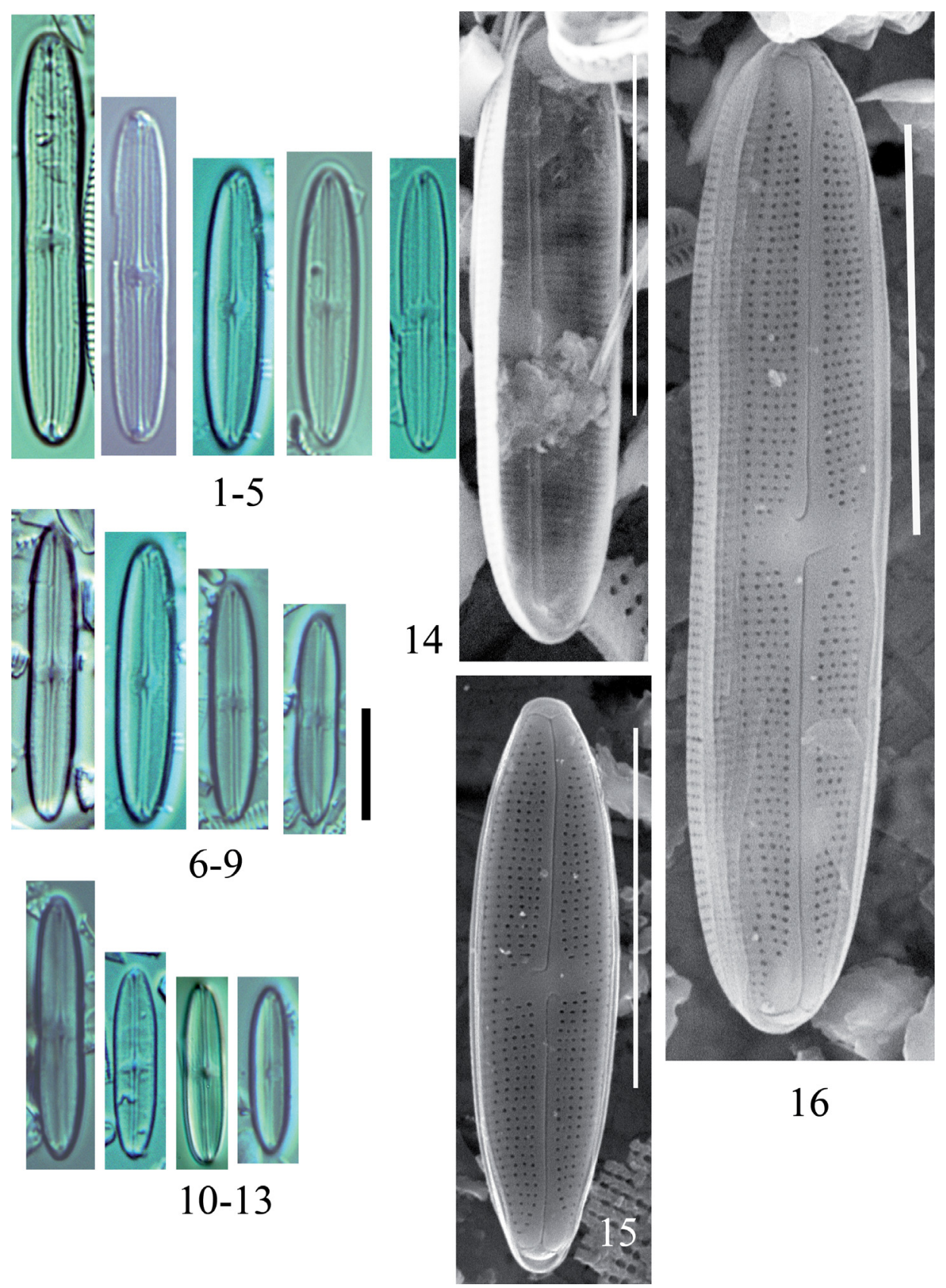

Plate 42: Neidium alpinum. - Figs 1-5: Lake Lezilor, LM. Figs 6-9: Lake Negru, LM. Figs 10-13: Lake Caprelor, LM. Fig. 14: Lake Caprelor, inside view, SEM. Fig. 15: Lake Caprelor, outside view, SEM. Fig. 16: Lake Lezilor, outside view, SEM. Scale bars $=10 \mu \mathrm{m}$. 
Neidium amphigomphus (Ehrenberg) Pfitzer 1871: 39

(Plate 43: Figs 1-2)

References: Pfitzer (1871), Siver et al. (2005).

Distribution in glacial lakes in the Retezat Mountains

\begin{tabular}{ll}
\hline Lakes & Brazi \\
Relative abundance $(\max )$. & $0.5 \%$ \\
Constancy & $1(4 \%)$ \\
\hline
\end{tabular}

Remarks: PÉTERFI (1993) reported this species from lakes, mires running waters as Neidium iridis var. amphigomphus. Very rare taxon. 

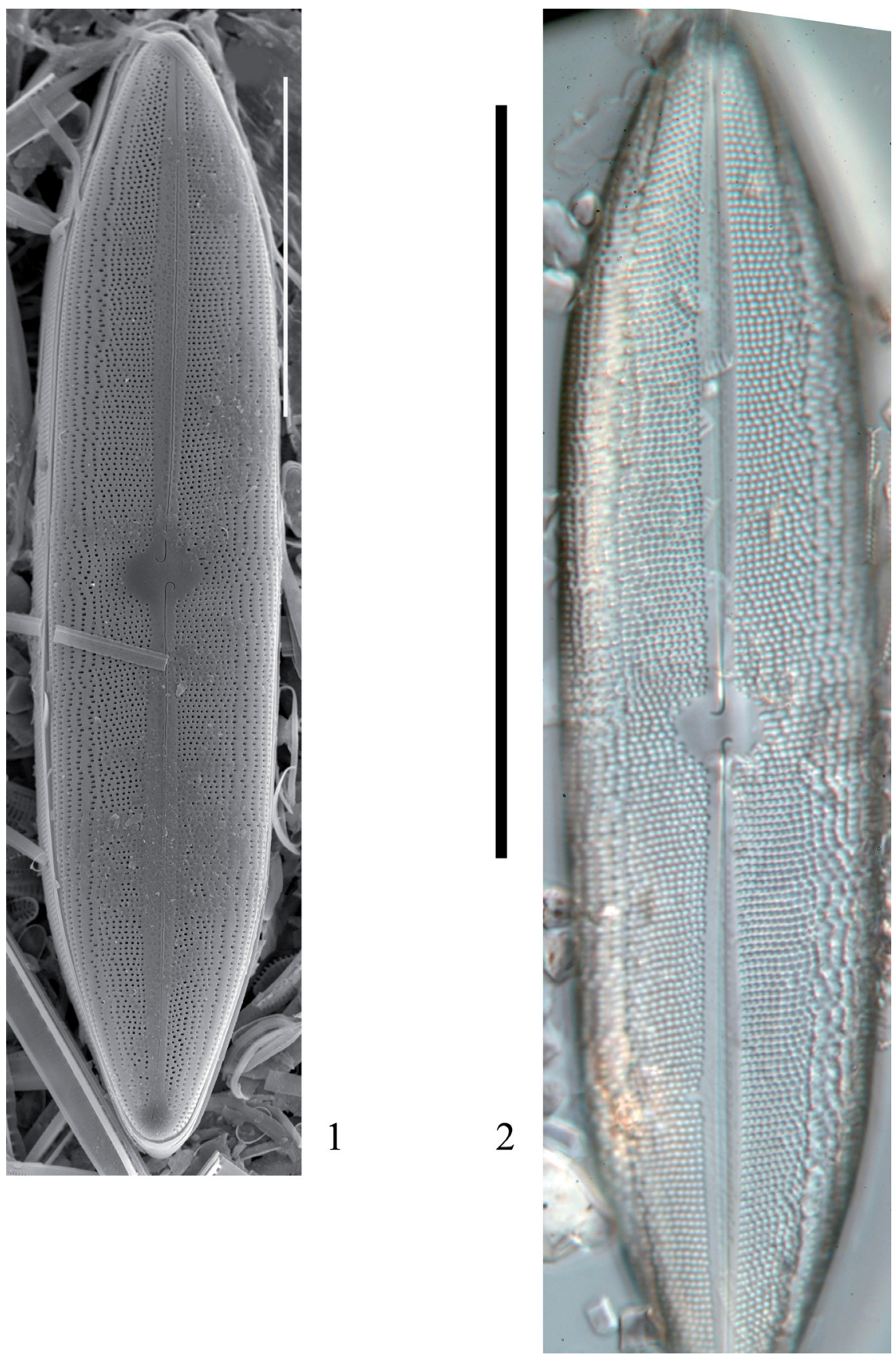

Plate 43: Neidium amphigomphus. - Fig. 1: Lake Brazi, outside view, SEM. Fig. 2: Lake Brazi, LM. Scale bars $=50 \mu \mathrm{m}$. 
Neidium ampliatum (Ehrenberg) Krammer in Krammer et LangeBertalot 1985: 101, pl. 2: figs 8-9, pl. 3: fig. 4 (Plate 44: Figs 1-5)

References: Krammer and Lange-Bertalot (1985), Hofmann et al. (2013).

Distribution in glacial lakes in the Retezat Mountains

\begin{tabular}{ll}
\hline Lakes & Caprelor, Florica, Gemenele, Pietrelice-1, Stanisoara \\
Relative abundance (max.) & $1 \%$ \\
Constancy & $2(22 \%)$ \\
\hline
\end{tabular}

Remarks: PÉTERFI (1993) reported this species from lakes and running waters as Neidium iridis var. ampliatum. This is a quite common species in our recent study. 

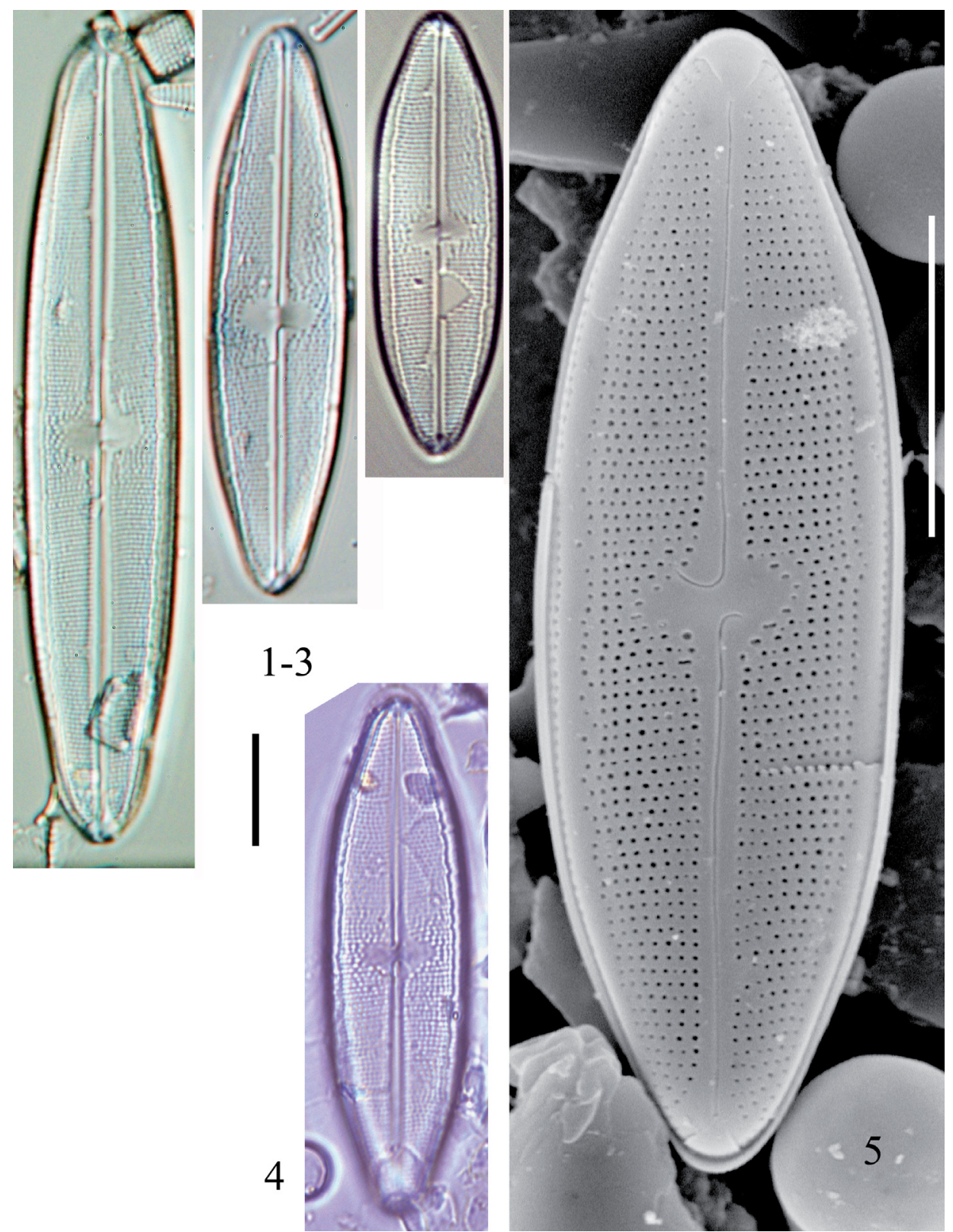

Plate 44: Neidium ampliatum. - Figs 1-3: Lake Brazi, LM. Fig. 4: Lake Negru, LM. Fig. 5: Lake Negru, outside view, SEM. Scale bars $=10 \mu \mathrm{m}$. 
Neidium cf. antarcticum P. B. Hamilton, M. de Haan, K. Kopalová, R. Zidarova et B. van de Vijver 2013: 32, figs 17-31 (Plate 45: Figs 1-11)

Reference: Hamilton et al. (2013).

Distribution in glacial lakes in the Retezat Mountains

\begin{tabular}{ll}
\hline Lakes & Bucura, Florica, Lia, Stanisoara, Stevia, Viorica \\
Relative abundance (max.) & $0.5 \%$ \\
Constancy & $2(26 \%)$ \\
\hline
\end{tabular}

Remark: This, recently described species is common in the mountain lakes of the Retezat Mts. Probably this is the second record of N. antactrica, it is know only from type locality so far. Dimensions: lengths $23-42 \mu \mathrm{m}$, width is $6-8 \mu \mathrm{m}$ and $28-30$ striae in $10 \mu \mathrm{m}$. 

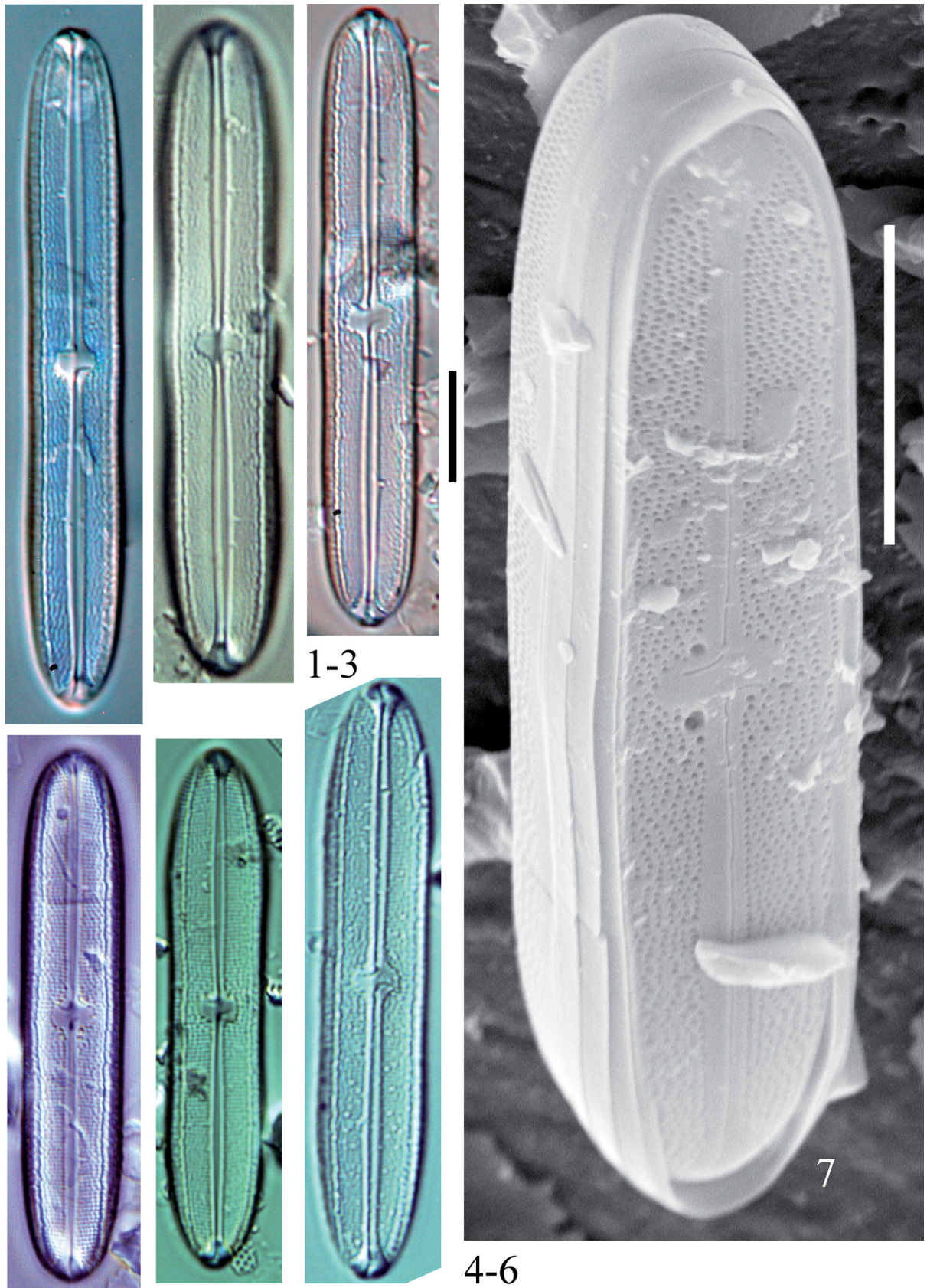

4-6

Plate 45: Neidium cf. antarcticum. - Figs 1-3: Lake Pelaguta, LM. Fig. 4: Lake Negru, LM. Figs 5-8: Lake Gales, LM. Figs 9-10: Lake Lia, LM. Fig. 11: Lake Lia, outside view, SEM. Scale bars $=10 \mu \mathrm{m}$. 
Neidium bisulcatum (Lagerstedt) Cleve 1894: 68 (Plate 46: Figs 1-7)

References: Cleve (1894), Krammer and LANge-Bertalot (1985), HoFMANN et al. (2013).

Distribution in glacial lakes in the Retezat Mountains

\begin{tabular}{ll}
\hline Lakes & Bucura, Gales, Lia, Viorica, Zanoaga \\
Relative abundance (max.) & $0.5 \%$ \\
Constancy & $2(22 \%)$ \\
\hline
\end{tabular}

Remarks: PÉTERFI (1993) reported this species from lakes and running waters. Not rare species. 


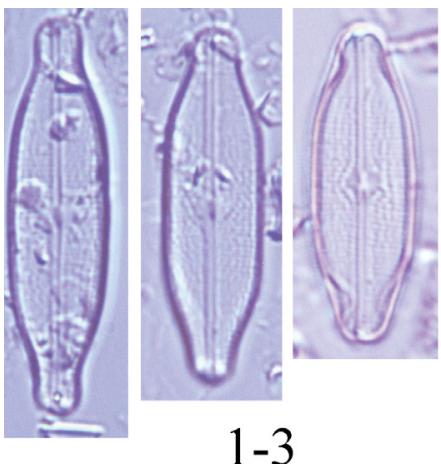

$1-3$
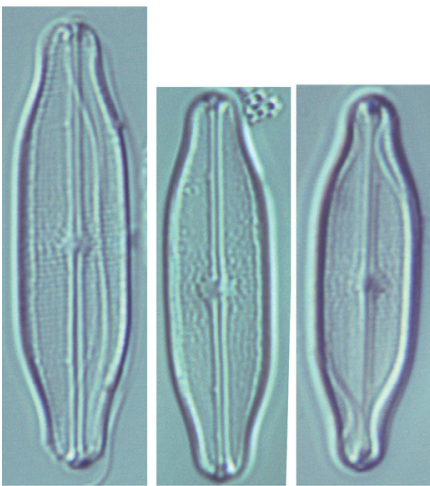

$5-8$

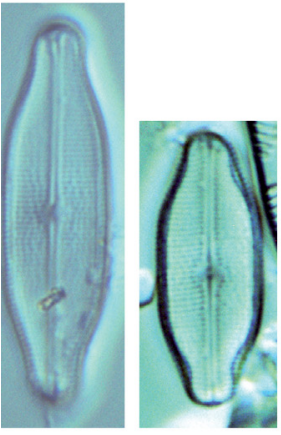

9-10
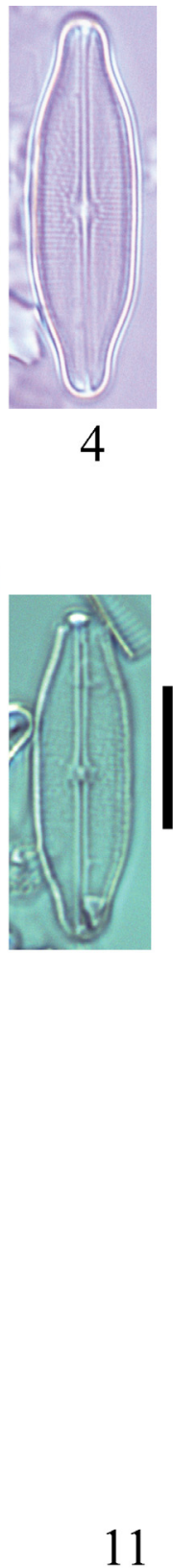

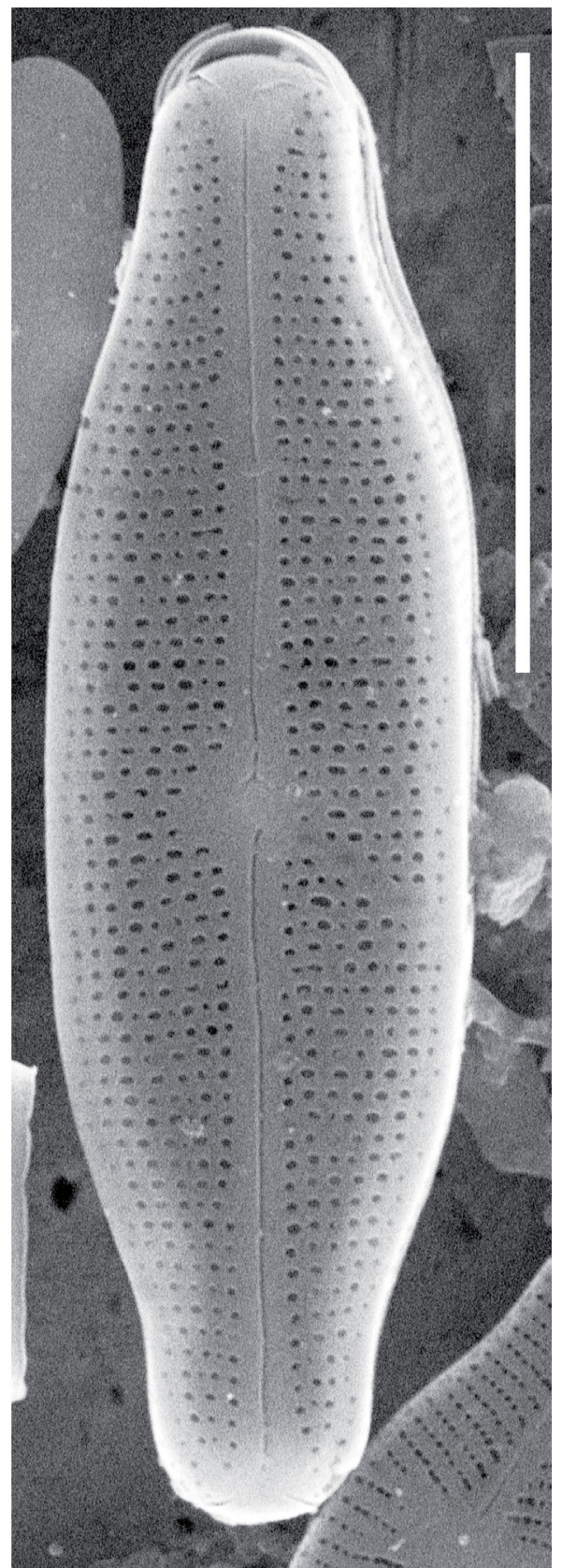

Plate 46: Neidium bisulcatum. - Figs 1-3: Lake Brazi, LM. Figs 4-6: Lake Negru, LM. Fig. 7: Lake Negru, outside view, SEM. Scale bars $=10 \mu \mathrm{m}$. 
Neidium continentale M. S. Kulikovskiy, Lange-Bertalot et A. Witkowski in Kulikovskiy, Lange-Bertalot, Witkowski, Dorofeyuk et Genkal 2010: 46, pl. 97: figs 1-3

(Plate 47: Figs 1-7)

Reference: KuLIKovsKiY et al. (2010).

Distribution in glacial lakes in the Retezat Mountains

\begin{tabular}{ll}
\hline Lakes & Lezilor \\
Relative abundance $(\max )$. & $0.5 \%$ \\
Constancy & $1(4 \%)$ \\
\hline
\end{tabular}

Remark: This species was only found in one lake in our recent study, very rare. 

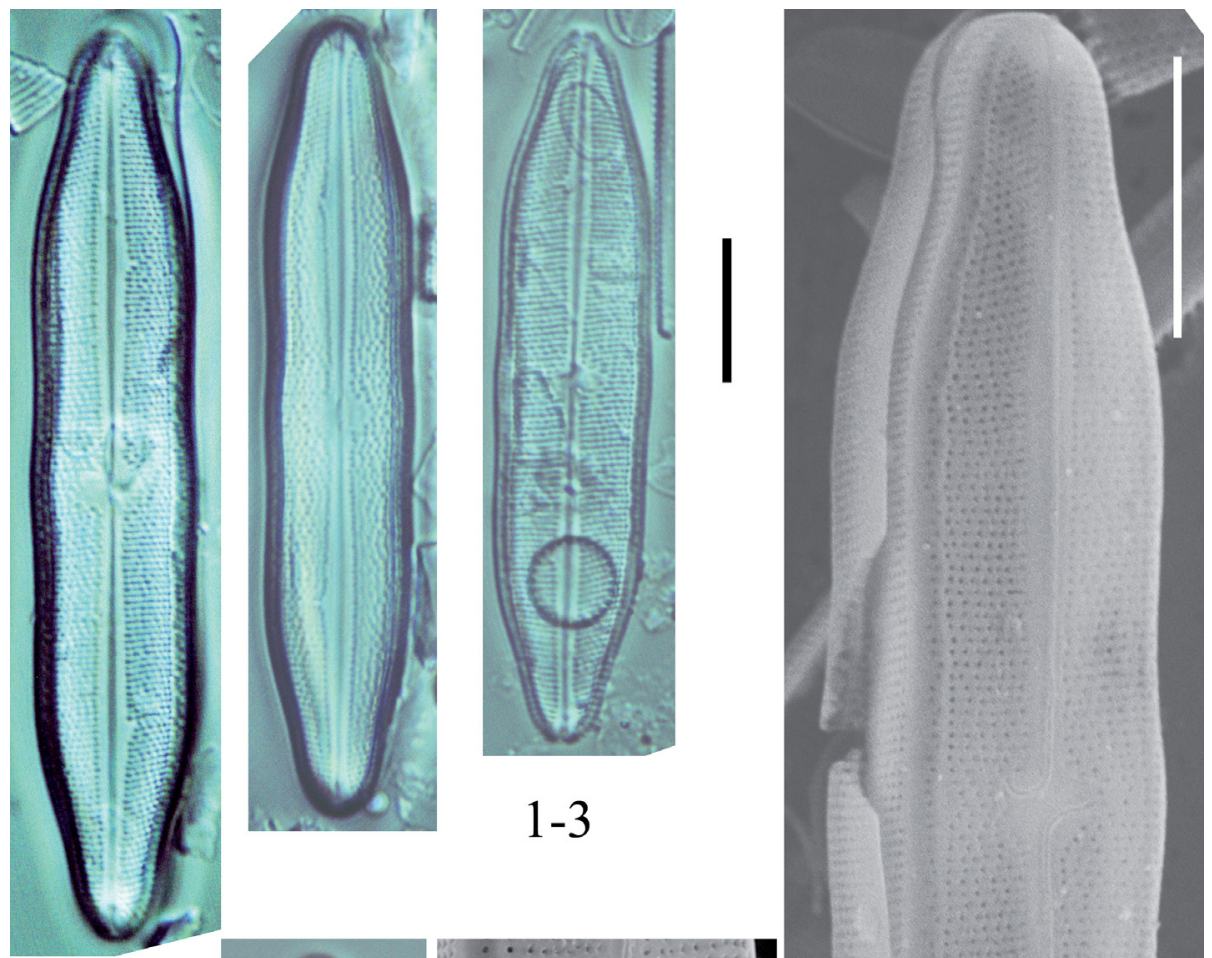

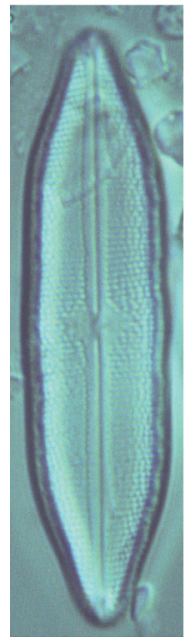

4

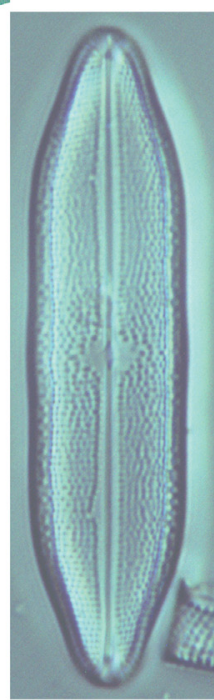

5

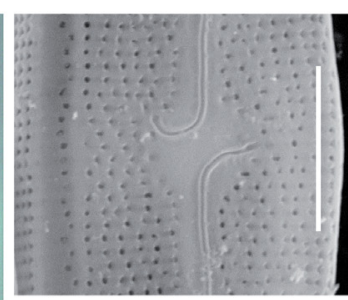

6

Plate 47: Neidium continentale. - Figs 1-5: Lake Lezilor, LM. Fig. 6: Lake Lezilor, outside view, central area, SEM. Fig. 7: Lake Lezilor, outside view, SEM. Scale bars $=10 \mu \mathrm{m}$ (Figs 1-5, 7), $5 \mu \mathrm{m}$ (Fig. 6). 
Neidium iridis (Ehrenberg) Cleve 1894: 69

(Plate 48: Figs 1-3)

References: CLEVE (1894), Siver et al. (2005).

Distribution in glacial lakes in the Retezat Mountains

\begin{tabular}{ll}
\hline Lakes & Brazi, Ana \\
Relative abundance (max.) & $0.5 \%$ \\
Constancy & $1(4 \%)$ \\
\hline
\end{tabular}

Remarks: PÉTERFI (1993) reported this species from lakes and running waters. Very rare. 


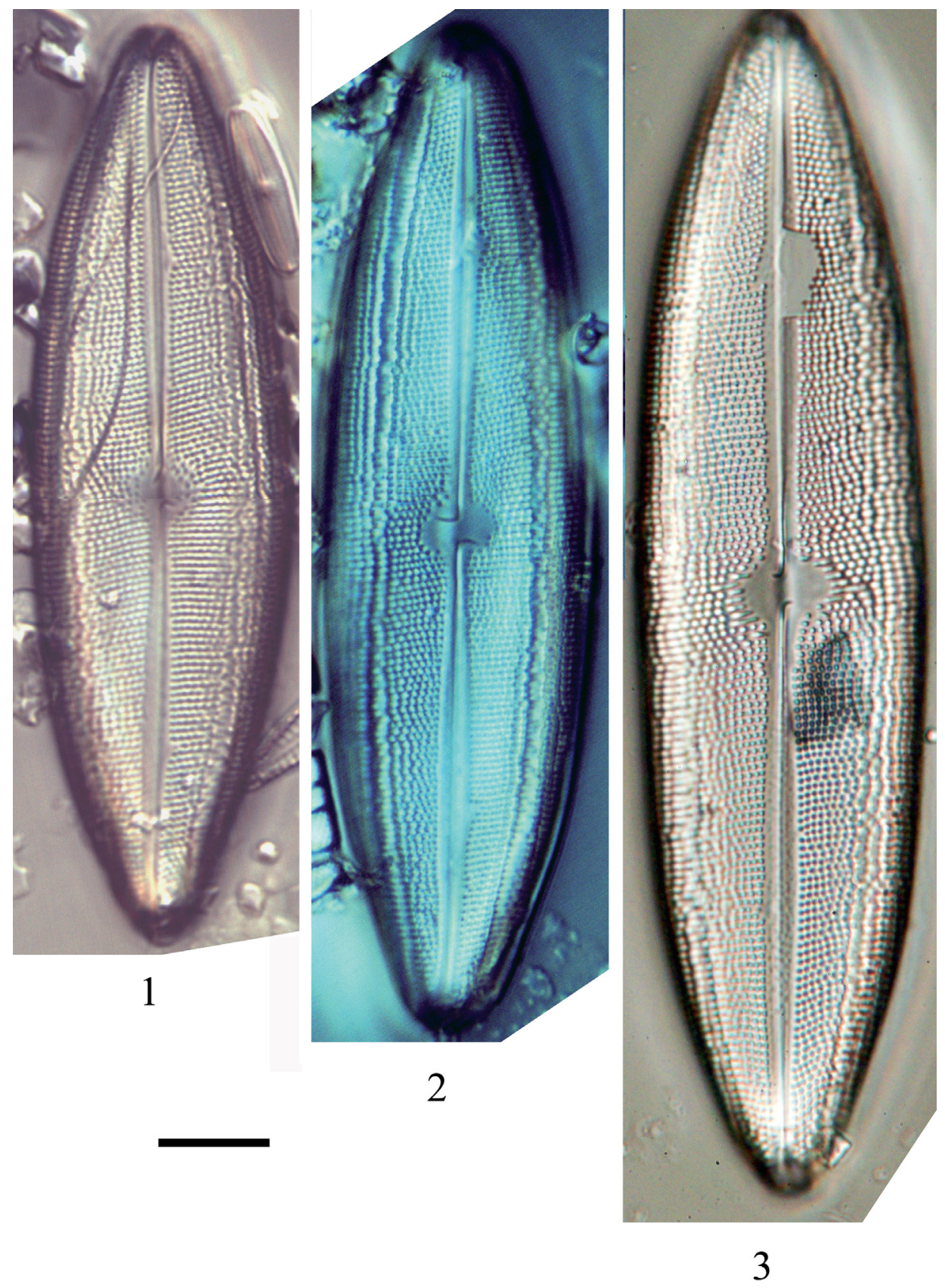

Plate 48: Neidium iridis. - Fig. 1: Lake Brazi, LM. Fig. 2: Lake Ana, LM. Fig. 3: Lake Brazi LM. Scale bars $=10 \mu \mathrm{m}$. 
Nitzschia perminuta (Grunow) M. Peragallo 1903: 672 (Plate 49: Figs 1-13)

References: Peragallo (1903), Kociolek (2011c), Hofmann et al. (2013).

Distribution in glacial lakes in the Retezat Mountains

\begin{tabular}{ll}
\hline Lakes & $\begin{array}{l}\text { Bucura, Caprelor, Florica, Gales, Peleguta, Stirbu, Turcelu, Vio- } \\
\text { rica, Zanoaga }\end{array}$ \\
Relative abundance (max.) & $2.5 \%$ \\
Constancy & $2(39 \%)$ \\
\hline
\end{tabular}

Remark: Quite common species in our recent study. 

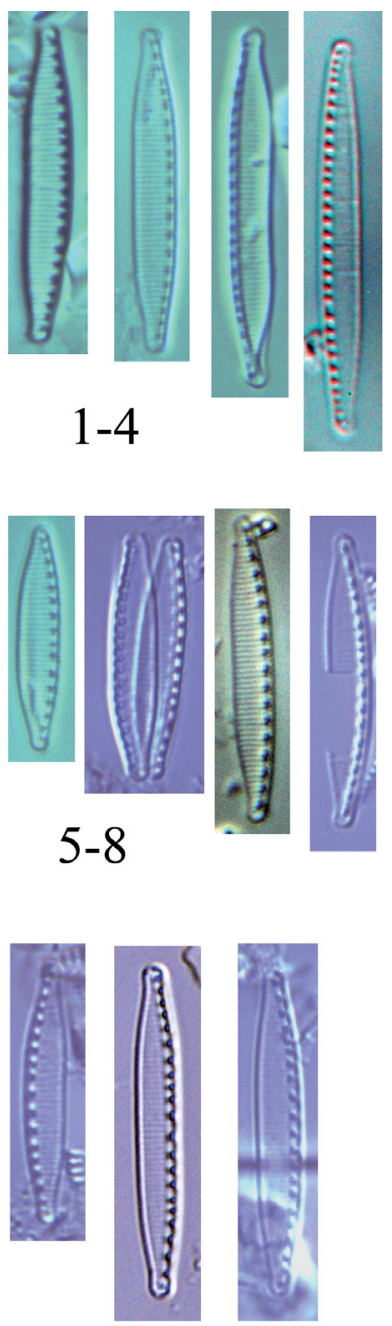

9-11

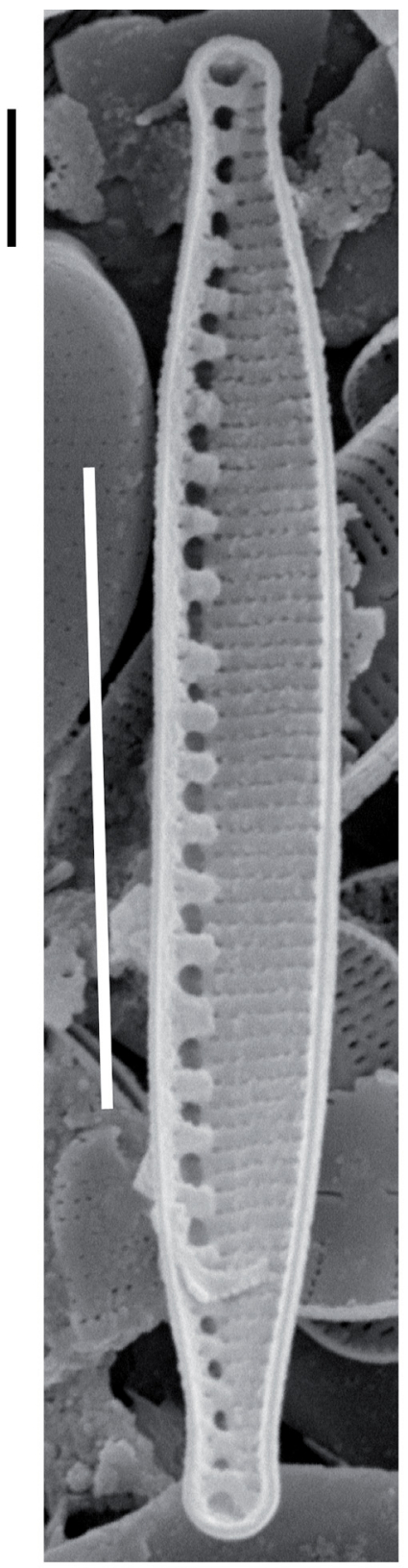

12

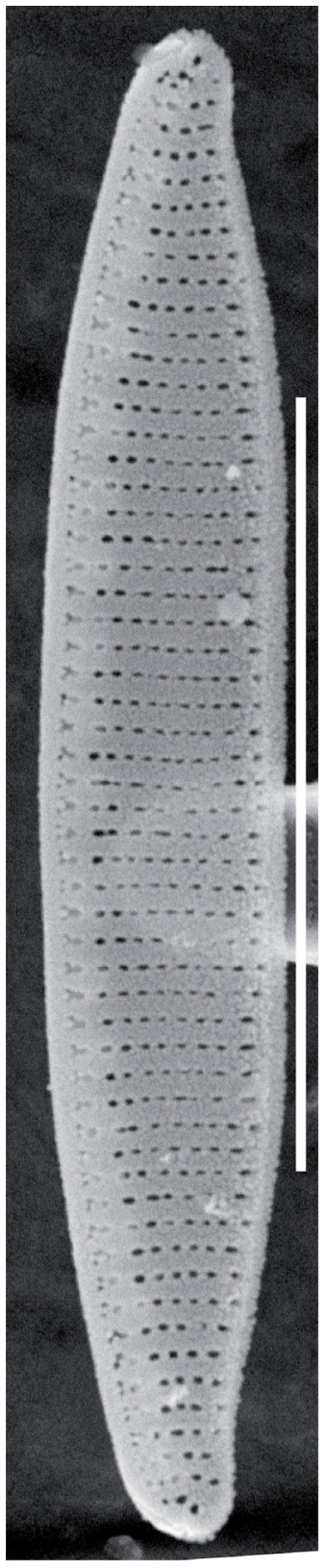

13

Plate 49: Nitzschia perminuta. - Figs 1-4: Lake Bucura, LM. Figs 5-8: Lake Pietrele, LM. Figs 9-1 1: Lake Slaviau, LM. Fig. 12: Lake Pietrele, inside view, SEM. Fig. 13: Lake Brazi, outside view, SEM. Scale bars $=10 \mu \mathrm{m}$. 
Nupela impexiformis (Lange-Bertalot) Lange-Bertalot 1999: 274 (Plate 50: Figs 1-13)

References: Krammer and LANge-Bertalot (1991), LANGe-Bertalot (1999a), Potapova (2011b).

Distribution in glacial lakes in the Retezat Mountains

\begin{tabular}{ll}
\hline Lakes & Brazi, Gales, Lia, Viorica \\
Relative abundance $(\max )$. & $0.5 \%$ \\
Constancy & $1(12 \%)$ \\
\hline
\end{tabular}

Remark: Never abundant, rare species. 

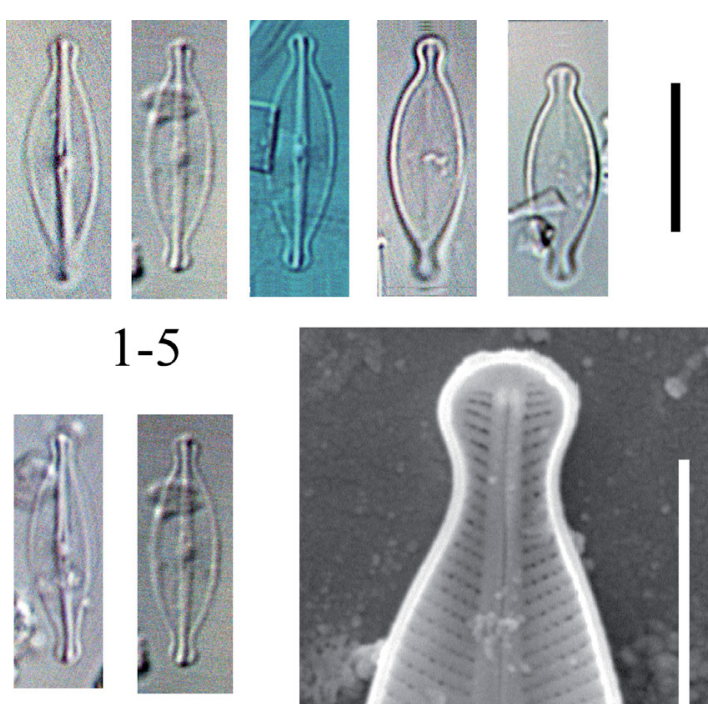

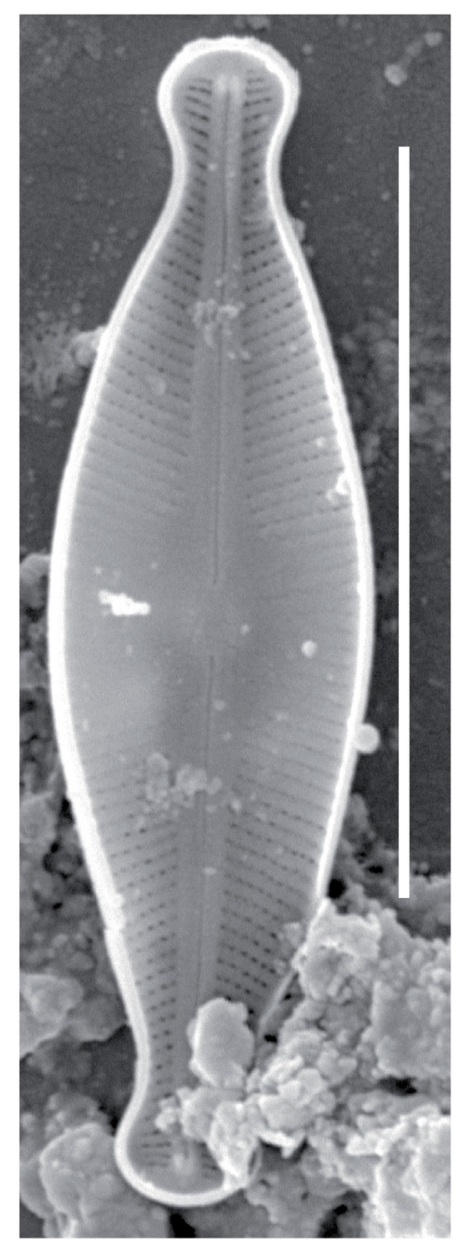

12

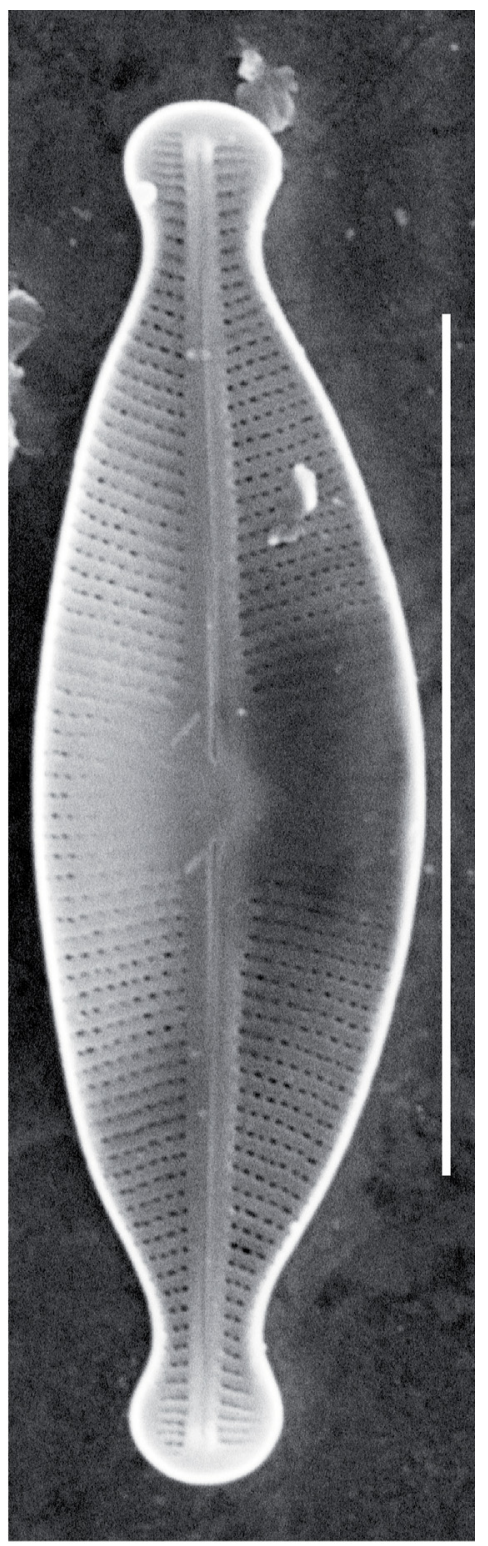

13

Plate 50: Nupela impexiformis. - Figs 1-7: Lake Brazi, LM. Figs 8-9: Lake Gales, LM. Figs 10-11: Lake Viorica, LM. Figs 12-13: Lake Gales, inside view, SEM. Scale bar = $10 \mu \mathrm{m}$ (Figs 1-11), $2 \mu \mathrm{m}$ (Figs 12-13). 


\section{Nupela lapidosa (Krasske) Lange-Bertalot 1999: 274}

(Plate 51: Figs 1-14)

References: LANGe-Bertalot (1999a), Potapova (2010a), Hofmann et al. (2013).

Distribution in glacial lakes in the Retezat Mountains

\begin{tabular}{ll}
\hline Lakes & Gales, Lia \\
Relative abundance $(\max )$. & $0.5 \%$ \\
Constancy & $1(8 \%)$ \\
\hline
\end{tabular}

Remark: Rare species. 

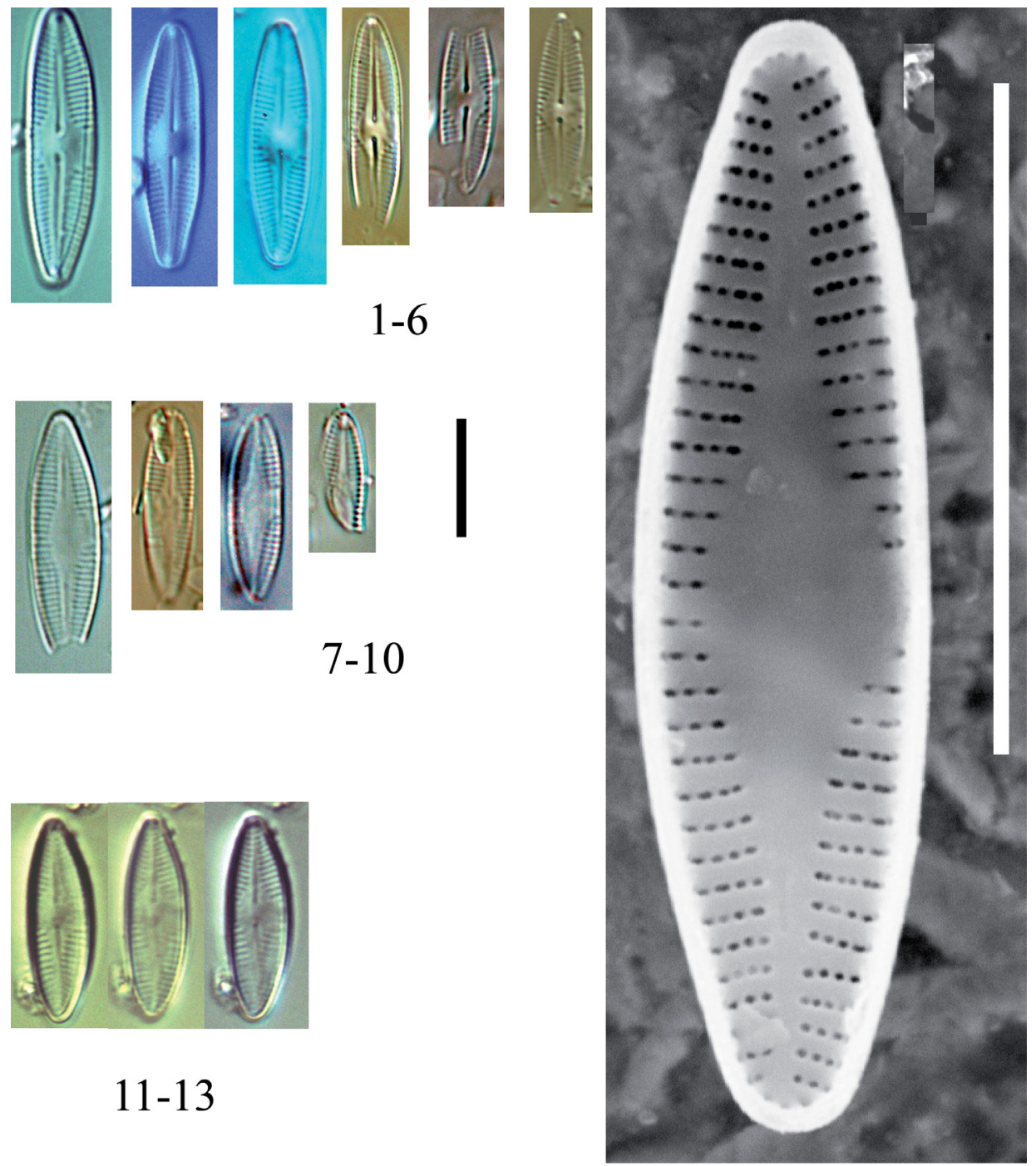

$$
11-13
$$

\section{4}

Plate 51: Nupela lapidosa. - Figs 1-6: Lake Gales, LM. Figs 7-10: Lake Lia, LM. Figs 11-13: Lake Gales, LM. Fig. 14: Lake Gales, inside view, SEM. Scale bars $=10 \mu \mathrm{m}$. 
Nupela paludigena (R. P. Scherer) Lange-Bertalot 1993: 158 (Plate 52: Figs 1-5)

References: LANGE-BERTALOT (1993), BuCZKó et al. (2013b).

Distribution in glacial lakes in the Retezat Mountains

\begin{tabular}{ll}
\hline Lakes & Zanoaga \\
Relative abundance $(\max )$. & $0.5 \%$ \\
Constancy & $1(4 \%)$ \\
\hline
\end{tabular}

Remark: This is a very rare species in our recent study, and also rare in Europe. 


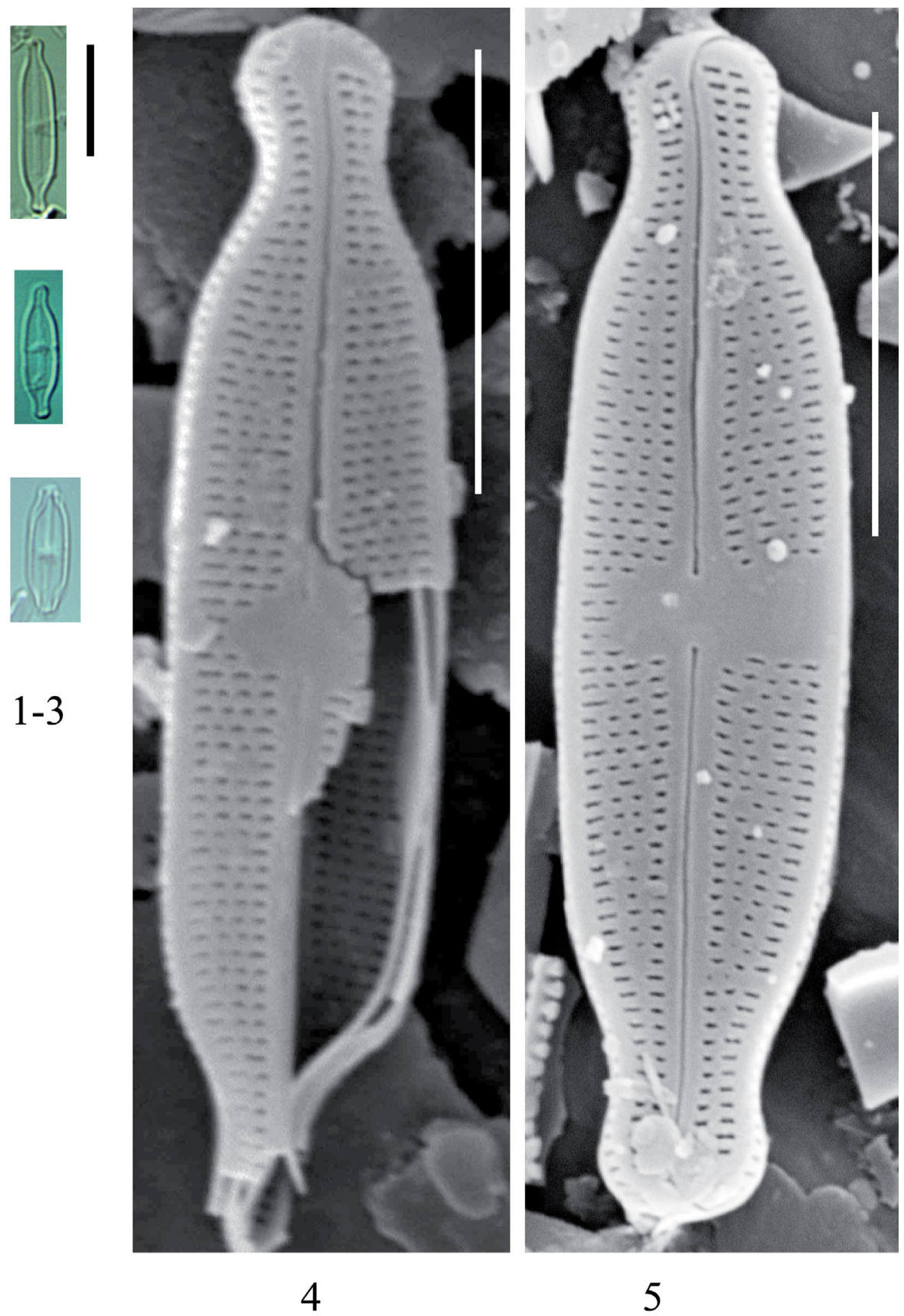

Plate 52: Nupela paludigena. - Figs 1-3: Lake Lia, LM. Figs 4-5: Lake Lia, outside view, SEM. Scale bars $=10 \mu \mathrm{m}$ (Figs 1-3), $5 \mu \mathrm{m}$ (Figs 4-5). 
Nupela pocsii K. Buczkó et A. Z. Wojtal in Buczkó, Wojtal et Magyari 2013: 432, fig. 28-38

(Plate 53: Figs 1-10)

References: BUCZKó et al. (2013b).

Distribution in glacial lakes in the Retezat Mountains

\begin{tabular}{ll}
\hline Lakes & Brazi \\
Relative abundance (max.) & $0.5 \%$ \\
Constancy & $1(4 \%)$ \\
\hline
\end{tabular}

Remark: Known only from the type locality. 

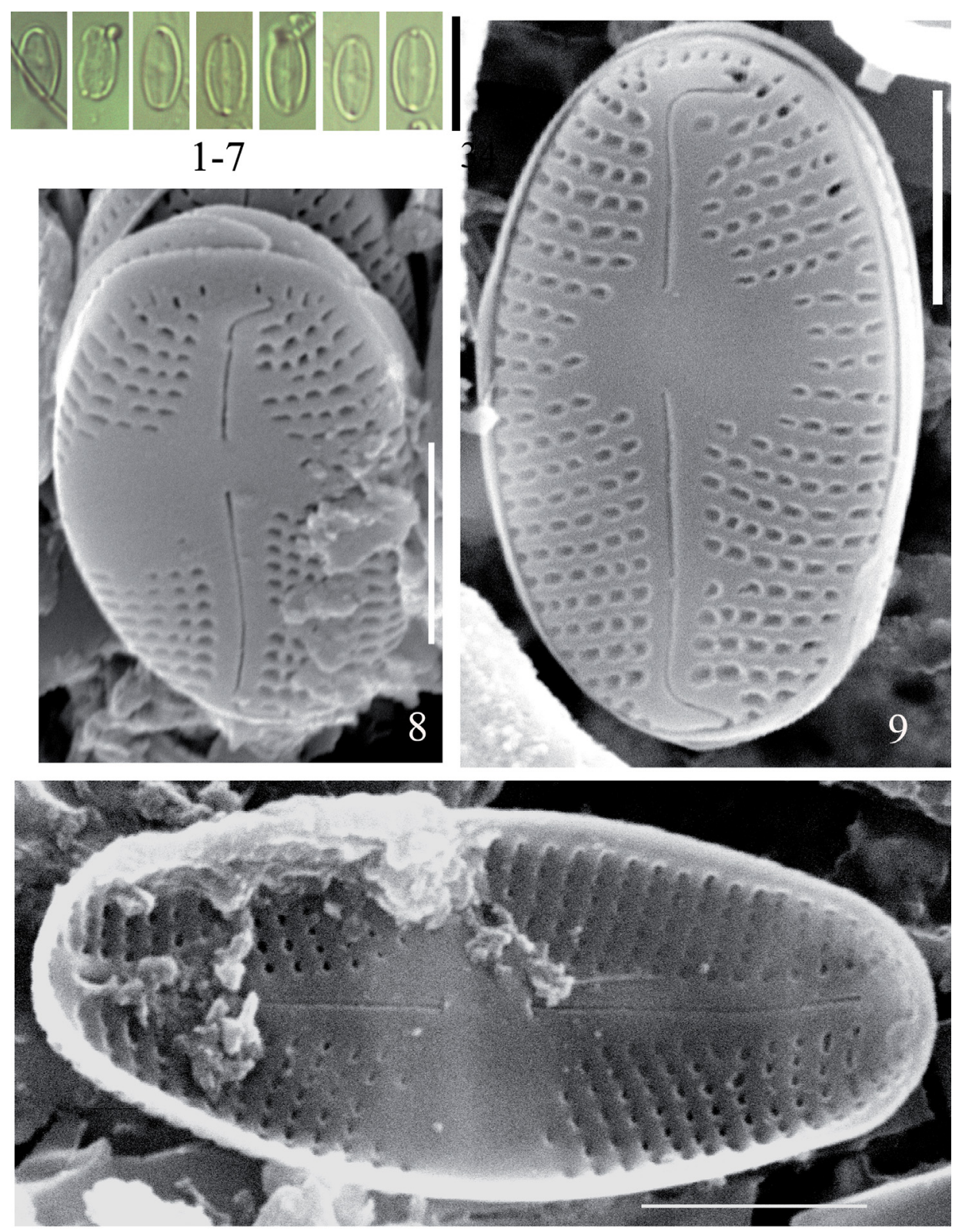

Plate 53: Nupela pocsii. - Figs 1-7: Lake Brazi, LM. Figs 8-9: Lake Brazi, outside view, SEM. Fig. 10: Lake Brazi, inside view, SEM. Scale bars $=10 \mu \mathrm{m}$ (Figs 1-7), $2 \mu \mathrm{m}$ (Figs 8-10). 
Nupela vitiosa (Schimanski) P. Siver et P. B. Hamilton 2005: 367 (Plate 54: Figs 1-10)

References: Siver and Hamilton (2005), Potapova (2010b).

Distribution in glacial lakes in the Retezat Mountains

\begin{tabular}{ll}
\hline Lakes & Brazi \\
Relative abundance $(\max )$. & $0.5 \%$ \\
Constancy & $1(4 \%)$ \\
\hline
\end{tabular}

Remark: Very rare. 


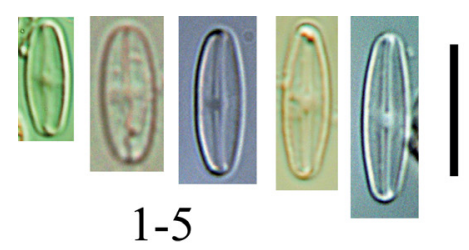

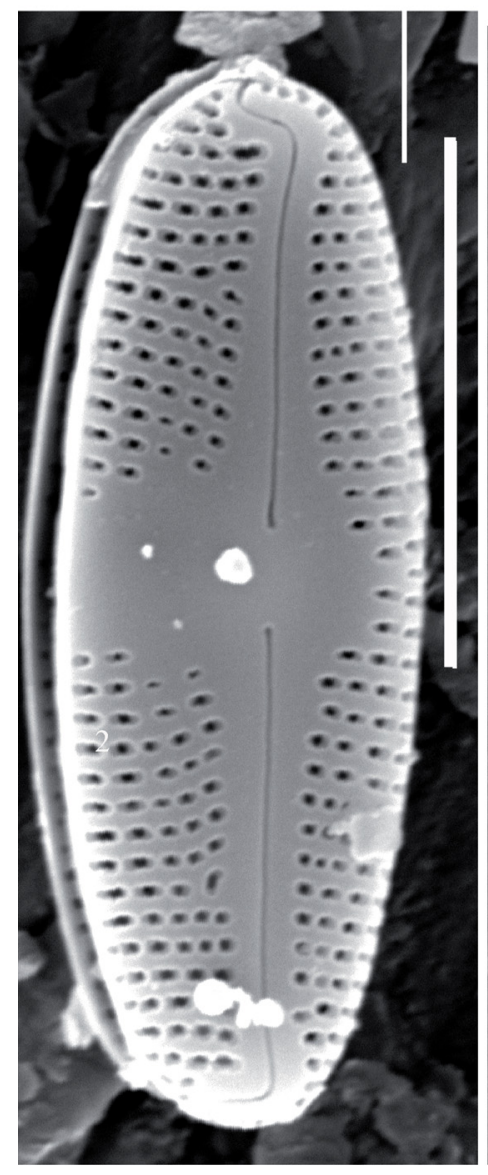

7
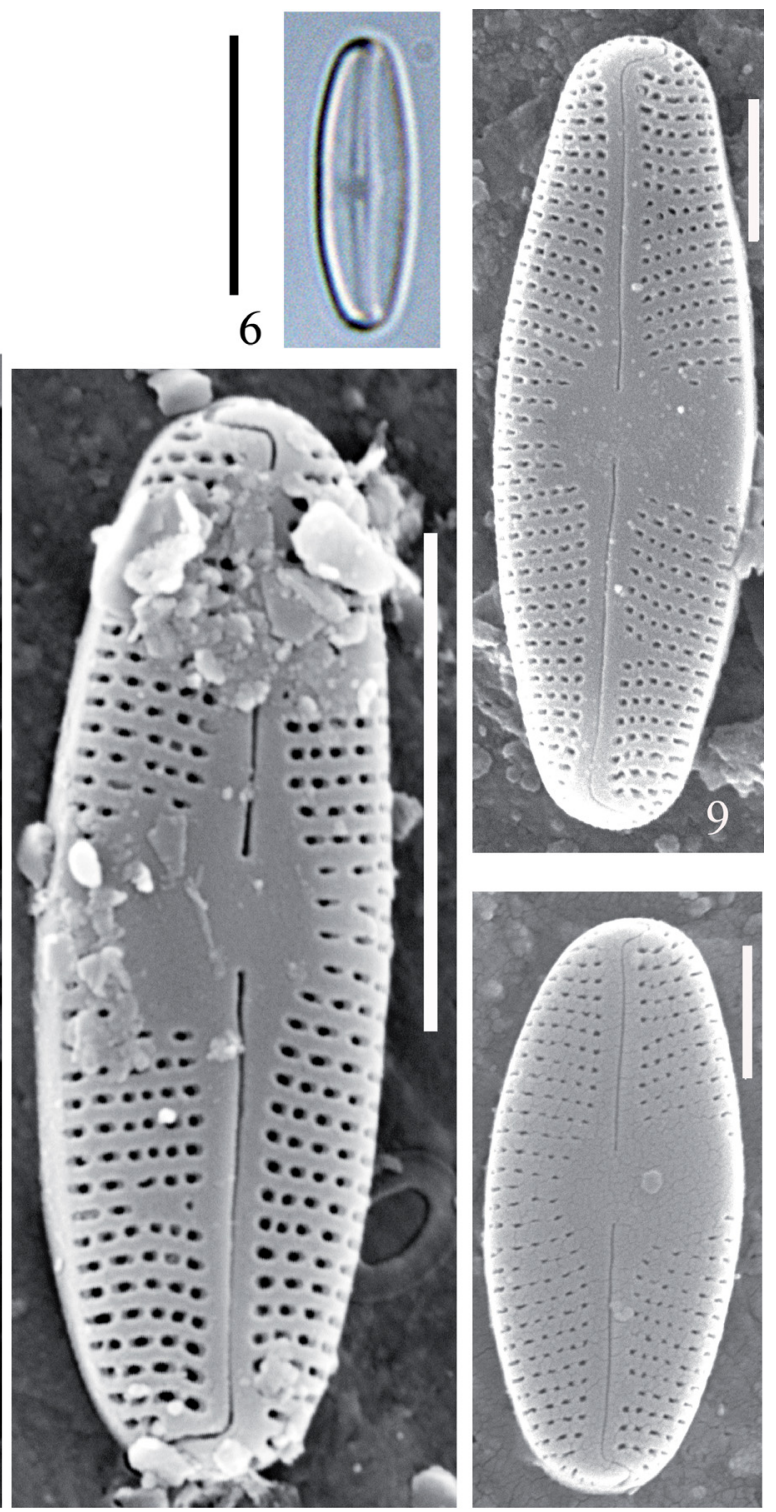

8
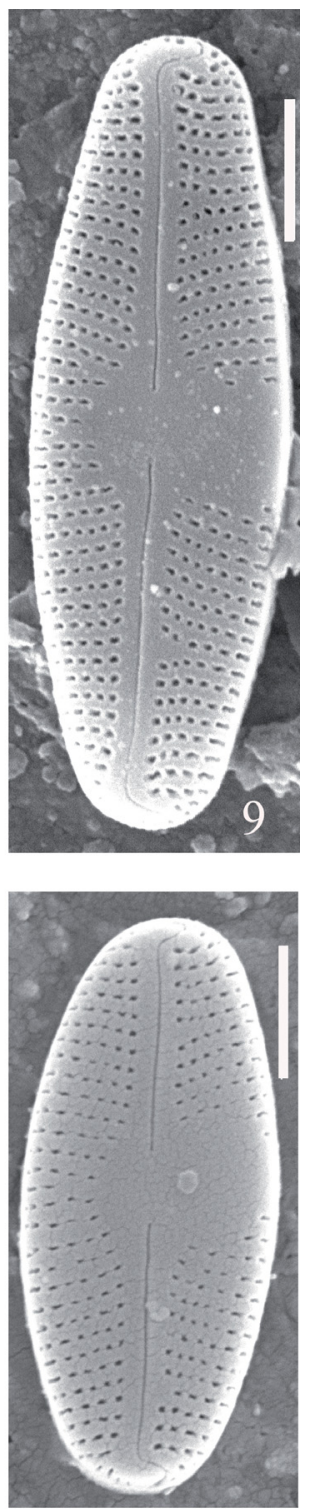

10

Plate 54: Nupela vitiosa. - Figs 1-6: Lake Brazi, LM. Figs 7-10: Lake Brazi, outside view, SEM. Scale bars $=10 \mu \mathrm{m}$ (Figs 1-6), $5 \mu \mathrm{m}$ (Figs 7-8), $2 \mu \mathrm{m}$ (Figs 9-10). 
Orthoseira roeseana (Rabenhorst) O'Meara 1876: 255

(Plate 55: Figs 1-7)

References: O'Meara (1875), Houk (1993, 2003), Hofmann et al. (2013).

Distribution in glacial lakes in the Retezat Mountains

Lakes

Lia, Negru, Peleguta, Zanoaga

Relative abundance (max.) $\quad 0.5 \%$

Constancy $1(16 \%)$

Remarks: PÉTERFI (1993) reported this species from running waters as $\mathrm{Me}$ losira roseana. This is a very rare taxon in our recent study. 

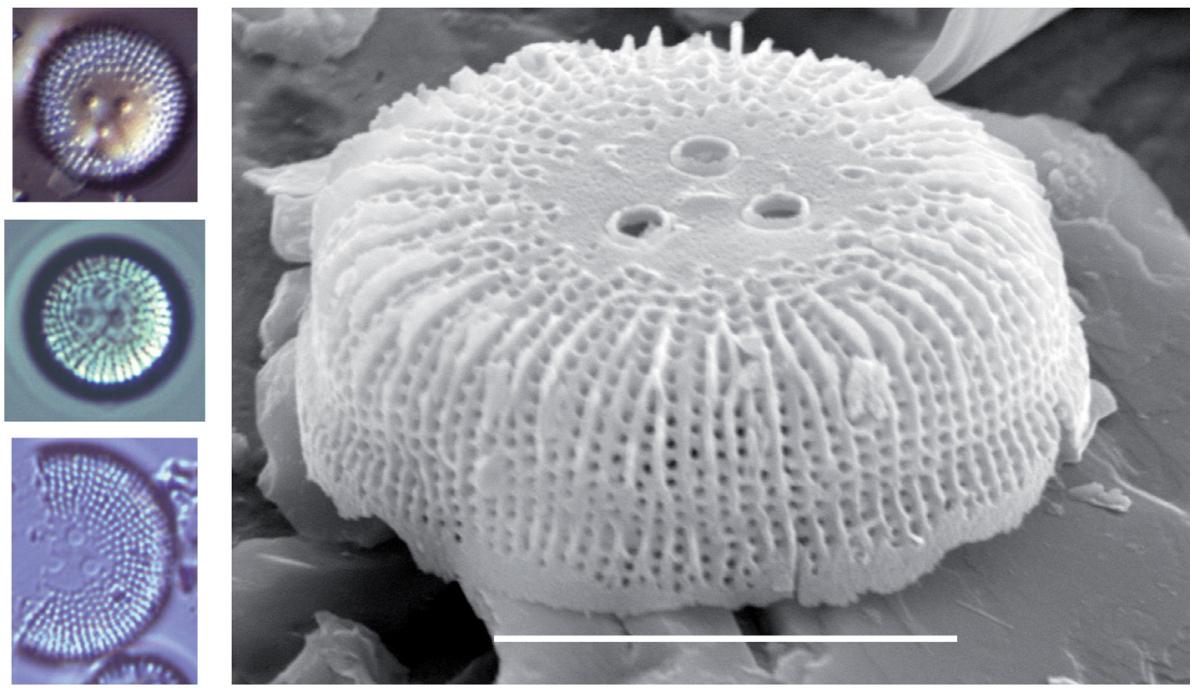

\section{$1-3$}

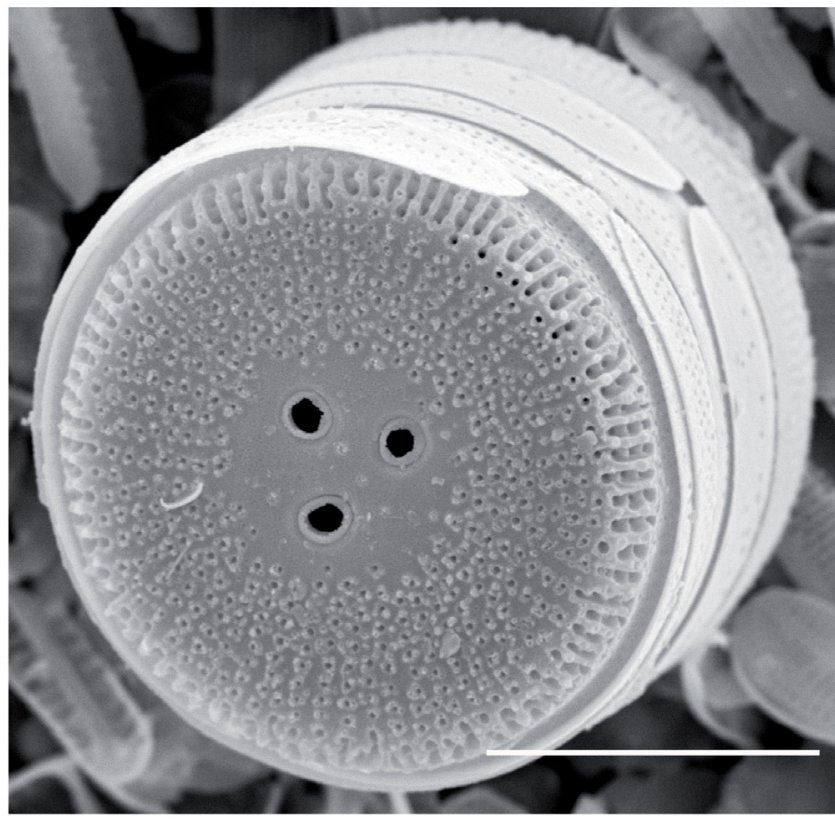

5
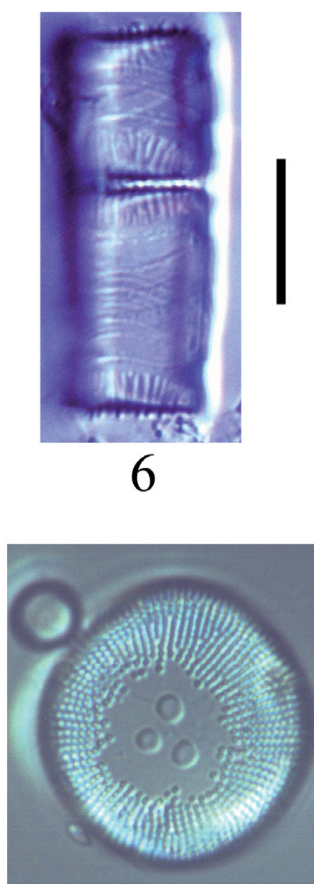

7

Plate 55: Orthoseira roeseana. - Figs 1-2: Lake Lia, LM. Fig. 3: Lake Peleguta, LM. Fig. 4: Lake Negru, outside view, SEM. Fig. 5: Lake Lia, outside view, SEM. Fig. 6: Lake Zanoaga, LM. Fig. 7: Lake Lia, LM. Scale bars $=10 \mu \mathrm{m}$. 
Planothidium distinctum (Messikommer) Lange-Bertalot 1999: 275

(Plate 56: Figs 1-11)

References: LANGE-BERTALOT (1999a), BuCzKó et al. (2013c).

Distribution in glacial lakes in the Retezat Mountains

\begin{tabular}{ll}
\hline Lakes & Lia \\
Relative abundance (max.) & $0.5 \%$ \\
Constancy & $1(4 \%)$ \\
\hline
\end{tabular}

Remark: Very rare species in the Retezat Mts. 


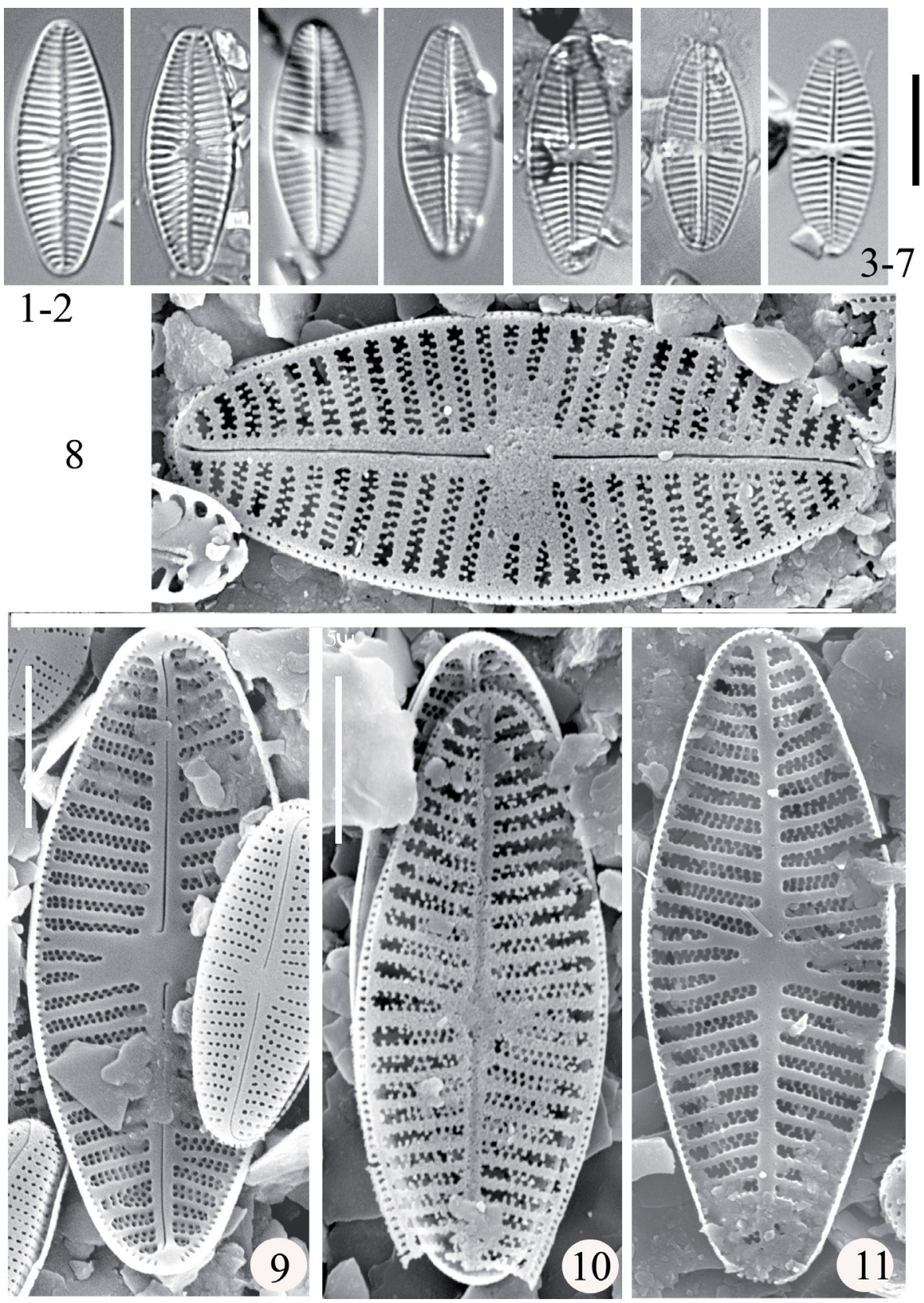

Plate 56: Planothidium distictum. - Figs 1-11: Lake Lia, LM. Figs 1-2: Rapheless valves, LM. Figs 3-7: Raphe valves, LM. Fig. 8: Raphe valve, outside view, SEM. Fig. 9: Raphe valve, inside view, SEM. Fig. 10: Rapheless valve, outside view, SEM. Fig. 11: Rapheless valve, inside view, SEM. Scale bars $=10 \mu \mathrm{m}$ (Figs 1-7), $5 \mu \mathrm{m}$ (Figs 8-11). 
Planothidium lanceolatum (Brébisson ex Kützing) Lange-Bertalot 1999: 287

(Plate 57: Figs 1-9)

References: Lange-Bertalot (1999a), Potapova (2010c), Hofmann et al. (2013).

Distribution in glacial lakes in the Retezat Mountains

\begin{tabular}{ll}
\hline Lakes & Lia \\
Relative abundance (max.) & $0.5 \%$ \\
Constancy & $1(4 \%)$ \\
\hline
\end{tabular}

Remarks: PÉTERFI (1993) reported this species from mires, lakes and running waters as Achnanthes lanceolata. Rare in our recent study. 

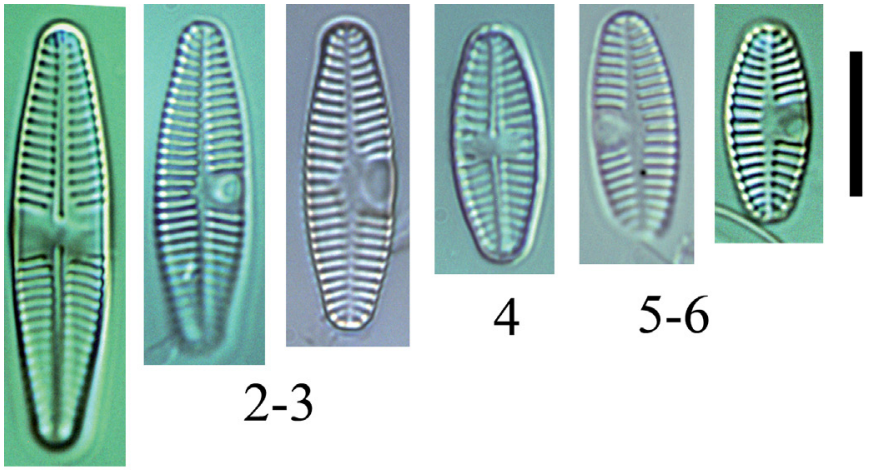

$2-3$

1

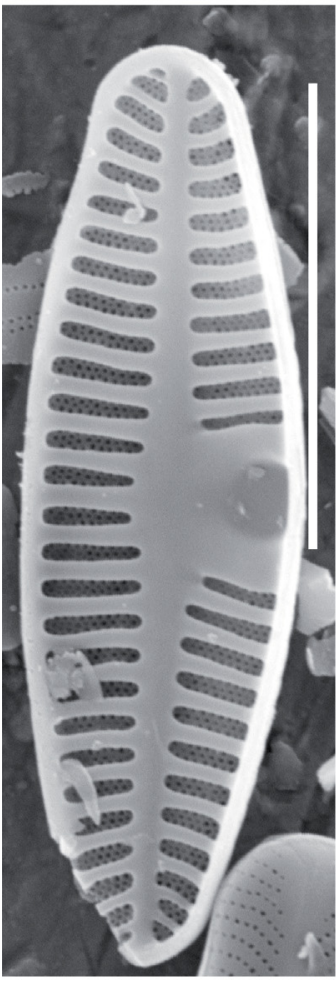

7

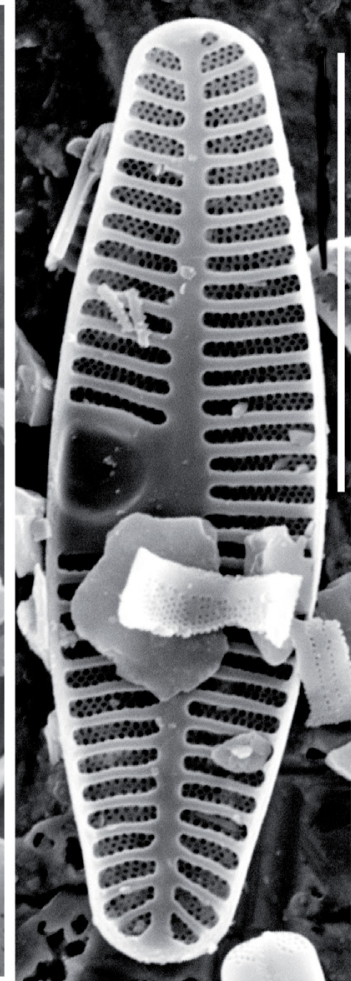

8

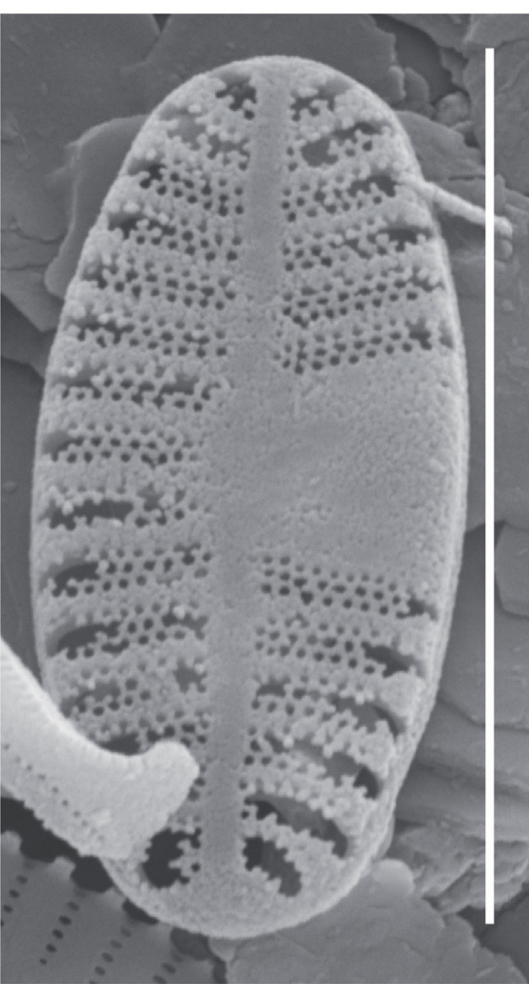

9

Plate 57: Planothidium lanceolatum. - Figs 1-9: Lake Lia, LM. Figs 1, 4: Raphe valves, LM. Figs 2-3, 5-6: Rapheless valves, LM. Figs 7-8: Lake Lia, rapheless valve, inside view, SEM. Fig. 9: Rapheless valve, outside view, SEM. Scale bars $=10 \mu \mathrm{m}$. 
Psammothidium altaicum (Poretzky) Bukhtiyarova in Bukhtiyarova et Round 1996: 5, figs 12-15

(Plate 58: Figs 1-9)

Reference: BuKHTIYAROVA and Round (1996).

Distribution in glacial lakes in the Retezat Mountains

\begin{tabular}{ll}
\hline Lakes & Brazi, Gales, Pietrele, Pietrelice-2, Viorica \\
Relative abundance (max.) & $1.3 \%$ \\
Constancy & $2(22 \%)$ \\
\hline
\end{tabular}

Remark: Rare species in our recent study. 

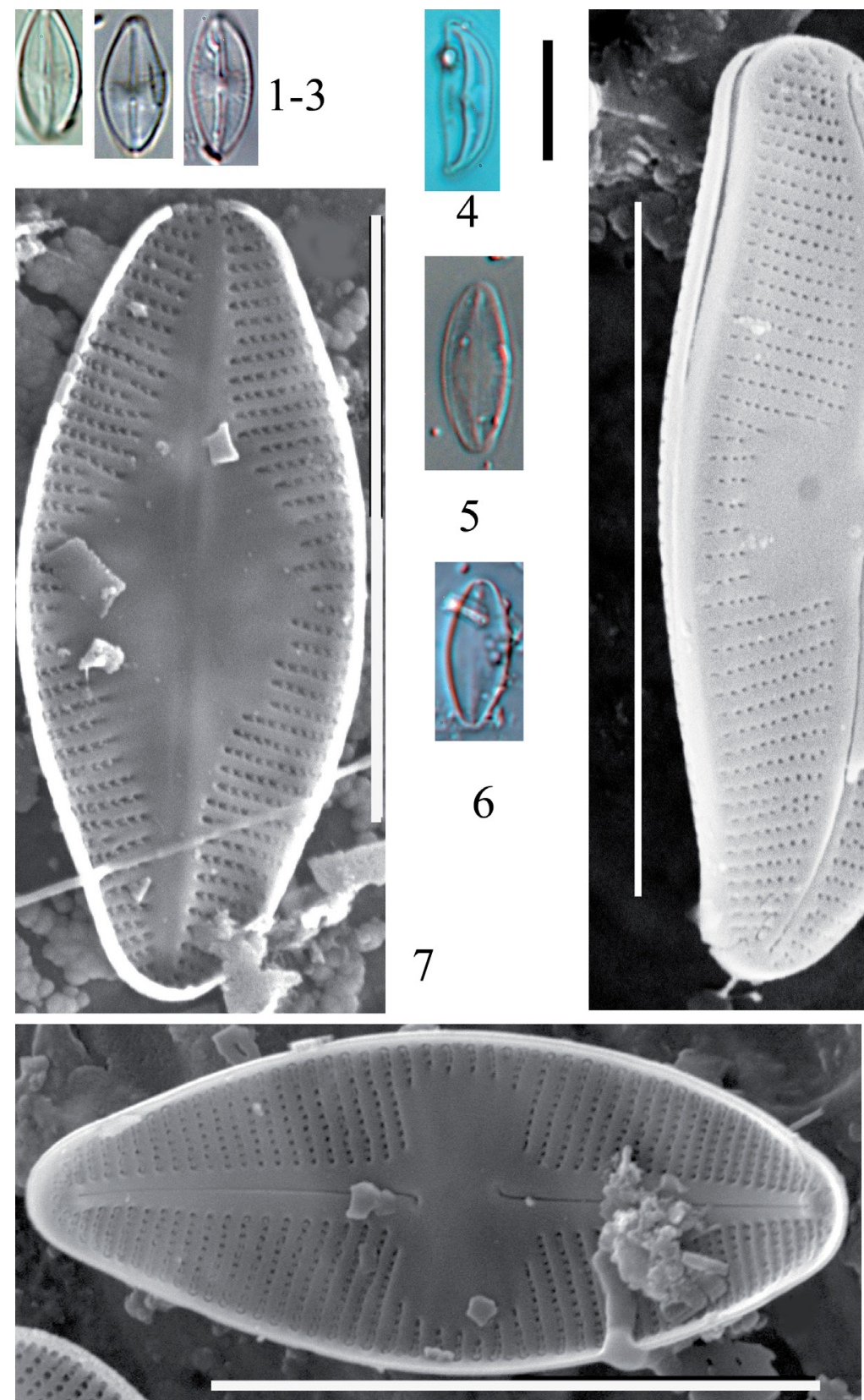

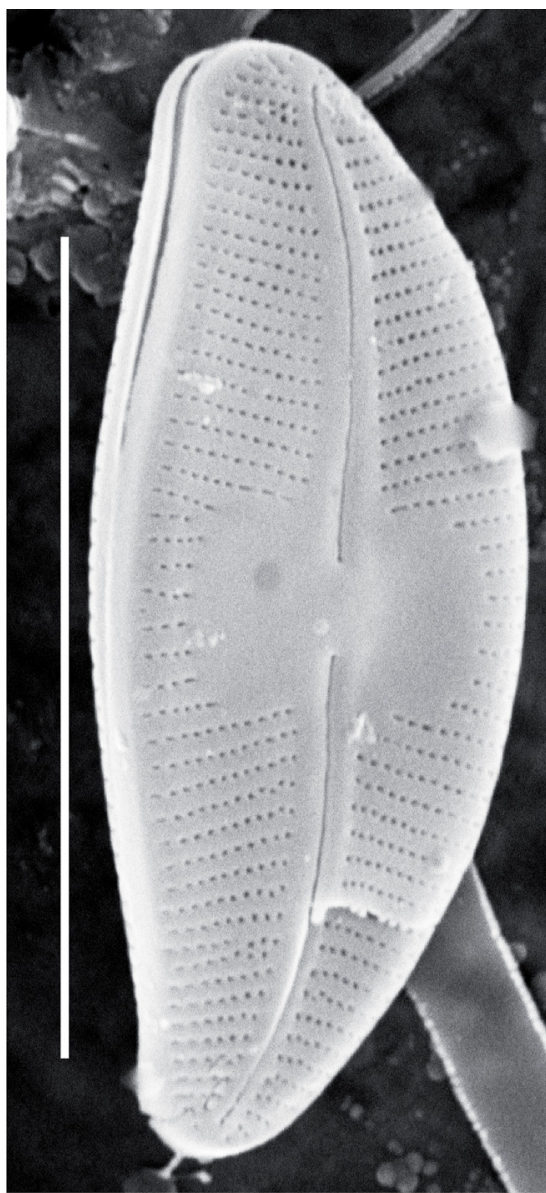

8

Plate 58: Psammothidium altaicum. - Figs 1-9: Lake Brazi. Figs 1-3: Raphe valve, LM. Fig. 4. Girdle view, LM. Figs 5-6: Rapheless valve, LM. Fig. 7: Rapheless valve, outside view, SEM. Fig. 8: Raphe valve, outside view, SEM. Fig. 9: Raphe valve, inside view, SEM. Scale bars $=10 \mu \mathrm{m}$. 
Psammothidium belveticum (Hustedt) L. N. Bukhtiyarova et Round 1996: 8, figs 20-25

(Plate 59: Figs 1-19)

References: Bukhtiyarova and Round (1996), Potapova (2010d), Hofmann et al. (2013).

Distribution in glacial lakes in the Retezat Mountains

\begin{tabular}{ll}
\hline Lakes & Ana, Brazi, Gemenele, Lia, Negru, Stanisoara, Stevia \\
Relative abundance (max.) & $5 \%$ \\
Constancy & $2(28 \%)$ \\
\hline
\end{tabular}



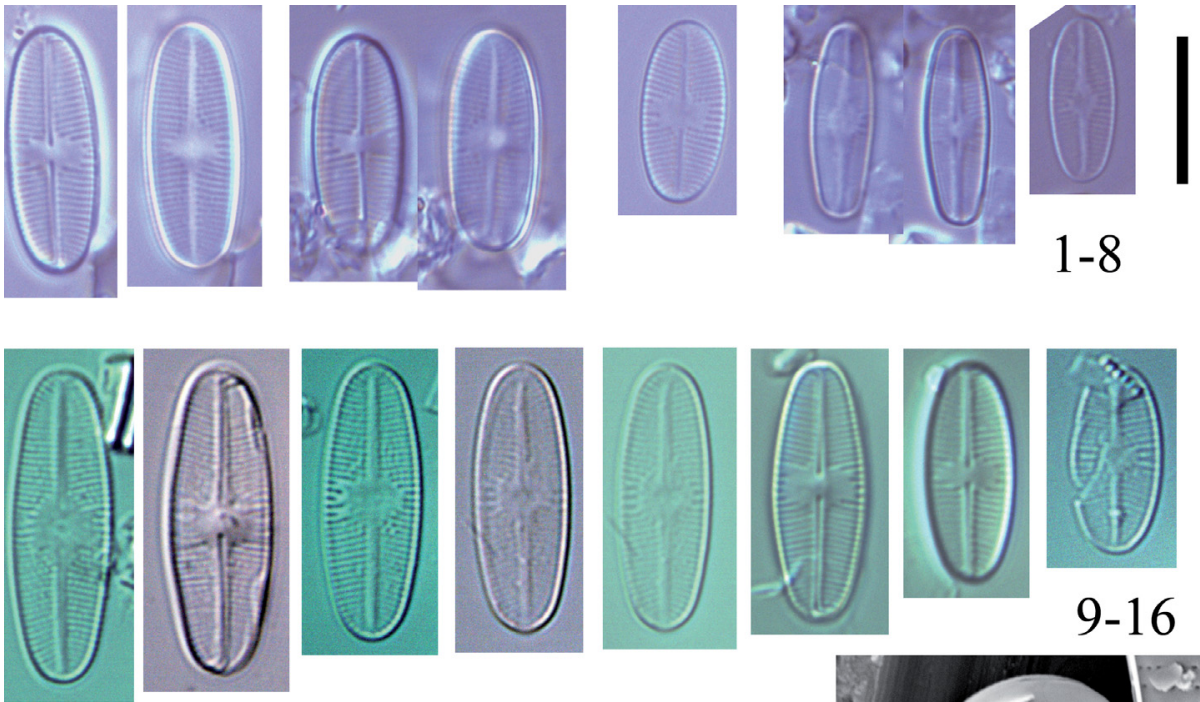

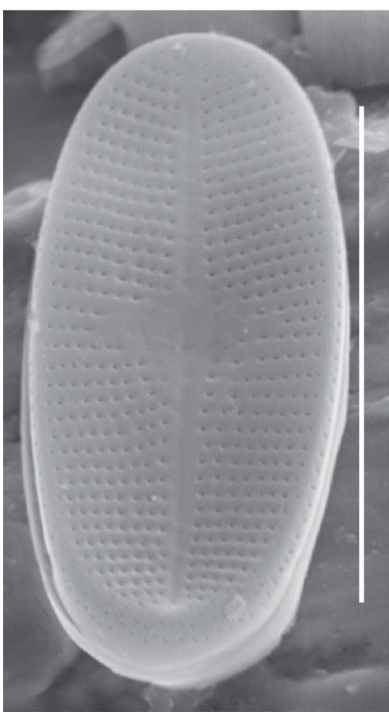

17

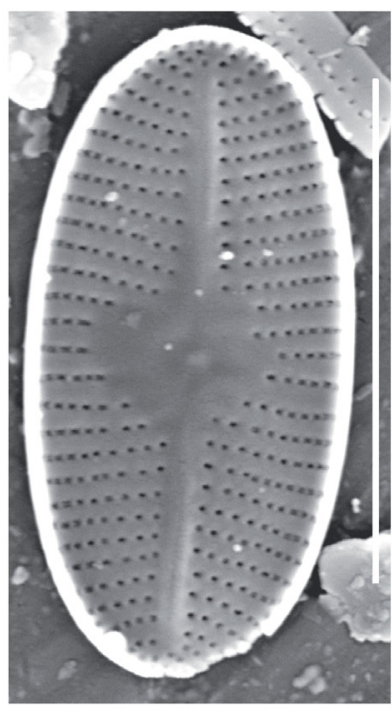

18

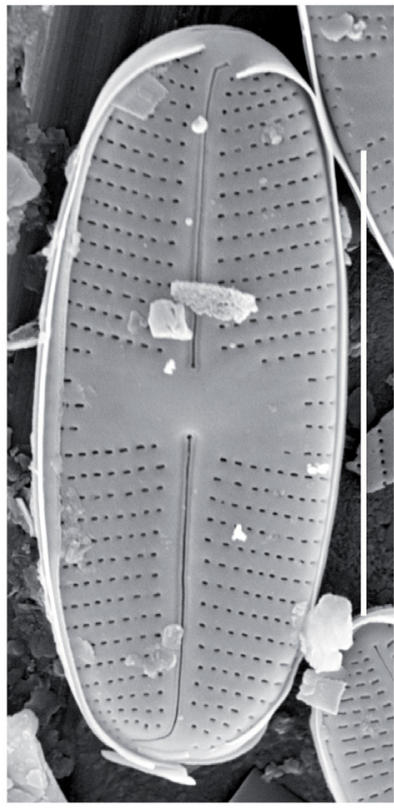

19

Plate 59: Psammothidium helveticum. - Figs 1-8: Lake Stevia, LM. Figs 9-16: Lake Lia, LM. Fig. 17: Lake Stevia, rapheless valve, SEM, outside view. Figs 18: Lake Stevia, rapheless valve, SEM, inside view. Fig. 19: Lake Lia, raphe valve, SEM, outside view. Scale bars $=10 \mu \mathrm{m}$. 


\section{Psammothidium belveticum var. minor (Flower et Jones) Buczkó, comb. nov. \\ (Plate 60: Figs 1-19)}

Basionym: Achnanthes helvetica var. minor Flower et Jones 1989, Diatom Research 11: 235, figs 19-25, 76-79.

Reference: Flower and JONES (1989)

Distribution in glacial lakes in the Retezat Mountains

\begin{tabular}{ll}
\hline Lakes & $\begin{array}{l}\text { Bucura, Caprelor, Florica, Gemenele, Lezilor, Lia, Peleaga, Pele- } \\
\text { guta, Slavieu, Stevia, Turcelu, Zanoaga }\end{array}$ \\
Relative abundance (max.) & $8 \%$ \\
Constancy & $3(52 \%)$ \\
\hline
\end{tabular}

Remarks: Valves are oval, length 8-11 $\mu \mathrm{m}$, width 5-6 $\mu \mathrm{m}$. Striae are 22-26 in $10 \mu \mathrm{m}$ on the raphe valves and on the rapheless $14-16$ in $10 \mu \mathrm{m}$. Common and sometimes abundant species in our recent study. 

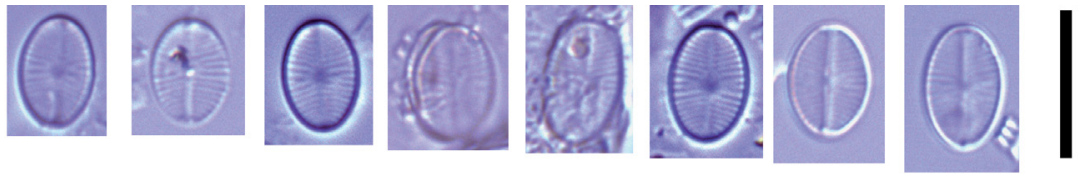

$1-8$
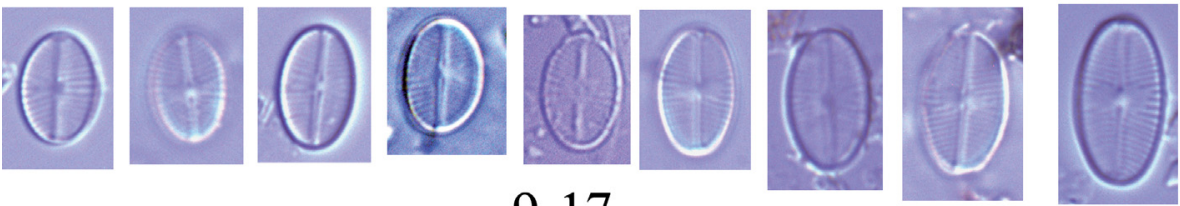

\section{9-17}

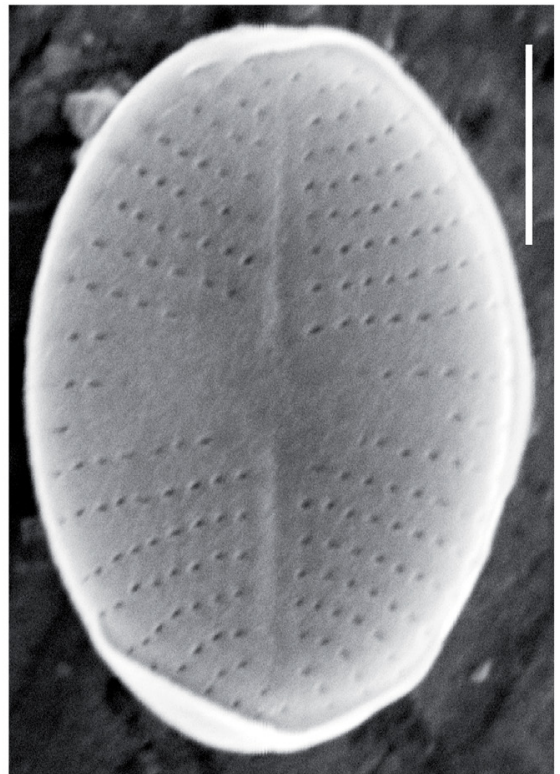

18

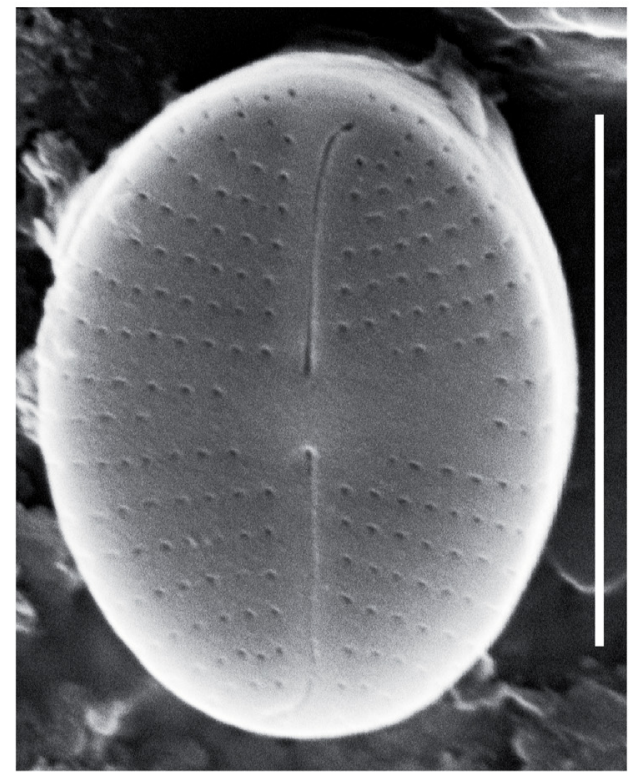

19

Plate 60: Psammothidium helveticum var. minor. - Figs 1-8: Lake Pietrelice, LM. Figs 9-17: Lake Stevia, LM. Fig. 18: Lake Pietrelice, rapheless valve, outside view, SEM. Fig. 19: Lake Pietrelice, raphe valve, outside view, SEM. Scale bars $=10 \mu \mathrm{m}$ (Figs 1-17), $5 \mu \mathrm{m}$ (Figs 18-19). 
Psammothidium kuelbsii (Lange-Bertalot) L. Bukhtiyarova et F. E. Round 1996: 16, fig. 61

(Plate 61: Figs 1-14)

Reference: BuKHTIYAROVA and Round (1996).

Distribution in glacial lakes in the Retezat Mountains

\begin{tabular}{ll}
\hline Lakes & Gemenele, Lezilor, Lia \\
Relative abundance (max.) & $1.5 \%$ \\
Constancy & $1(12 \%)$ \\
\hline
\end{tabular}

Remarks: The dimensions and shape of this species resemble to Navicula submitis, that is published by PÉTERFI (1993) in glacial lakes. Rare species in our recent study. 

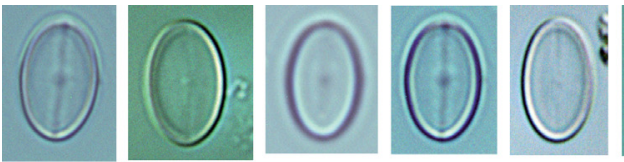

$1-6$
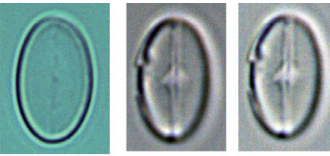

$7-8$
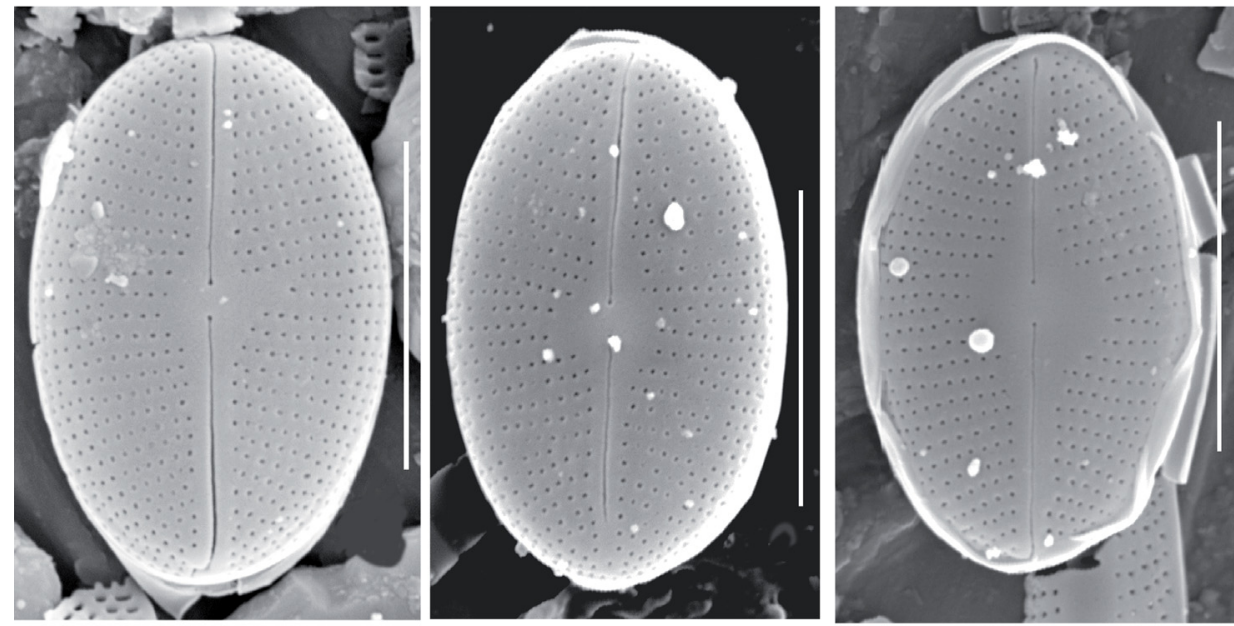

9-11

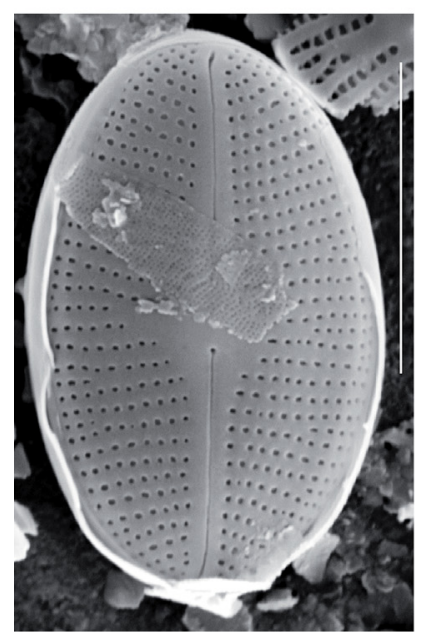

12

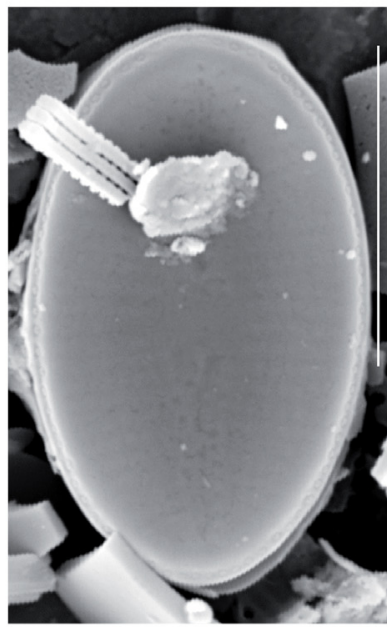

13

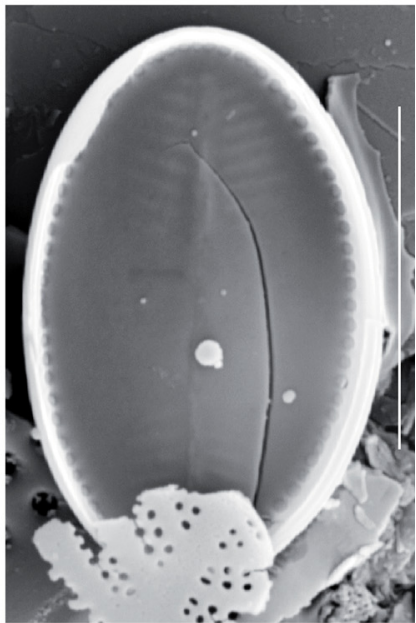

14

Plate 61: Psammothidium kuelbsii. - Figs 1-10: Lake Lia, LM. Figs 1-6: Rapheless valve. Figs 7-8: Raphe valve. Figs 9-12: SEM outside view, raphe valve. Fig. 13: SEM, rapheless valve, outside view. Fig. 14: SEM raphe valve, inside view. Scale bar $=10 \mu \mathrm{m}$ (Figs 1-8), $5 \mu \mathrm{m}$ (Figs 9-14). 
Psammothidium levanderi (Hustedt) Bukhtiyarova et Round 1996: 18 , figs 66-67

(Plate 62: Figs 1-9)

References: Bukhtiyarova and Round (1996), Potapova (2010e).

Distribution in glacial lakes in the Retezat Mountains

\begin{tabular}{ll}
\hline Lakes & Lia \\
Relative abundance (max.) & $2 \%$ \\
Constancy & $1(4 \%)$ \\
\hline
\end{tabular}

Remark: Rare and not abundant taxon in our recent study. 


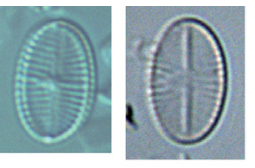

$1-2$
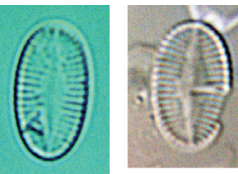

(4)

$3-7$
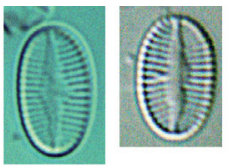

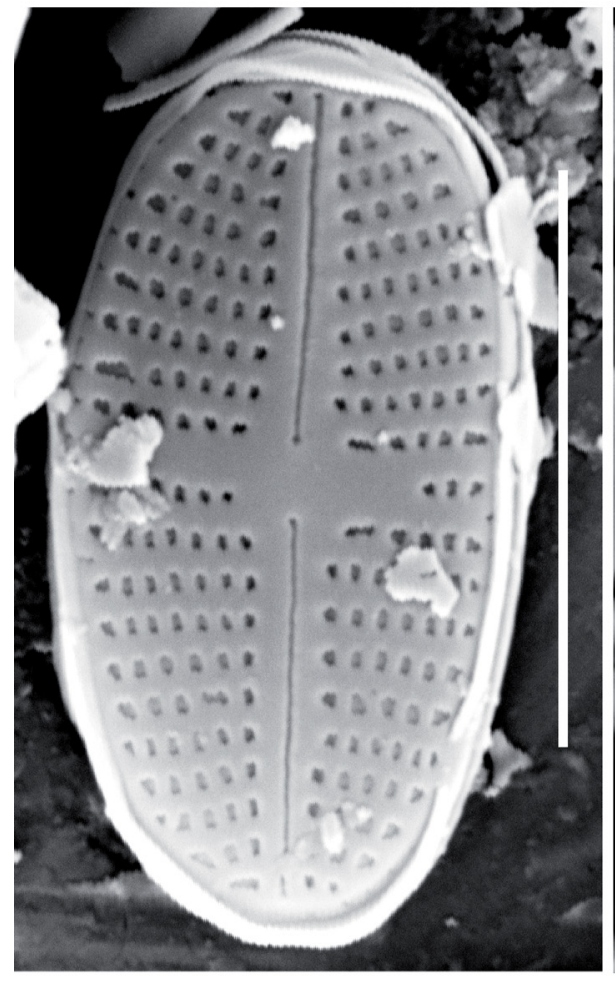

8

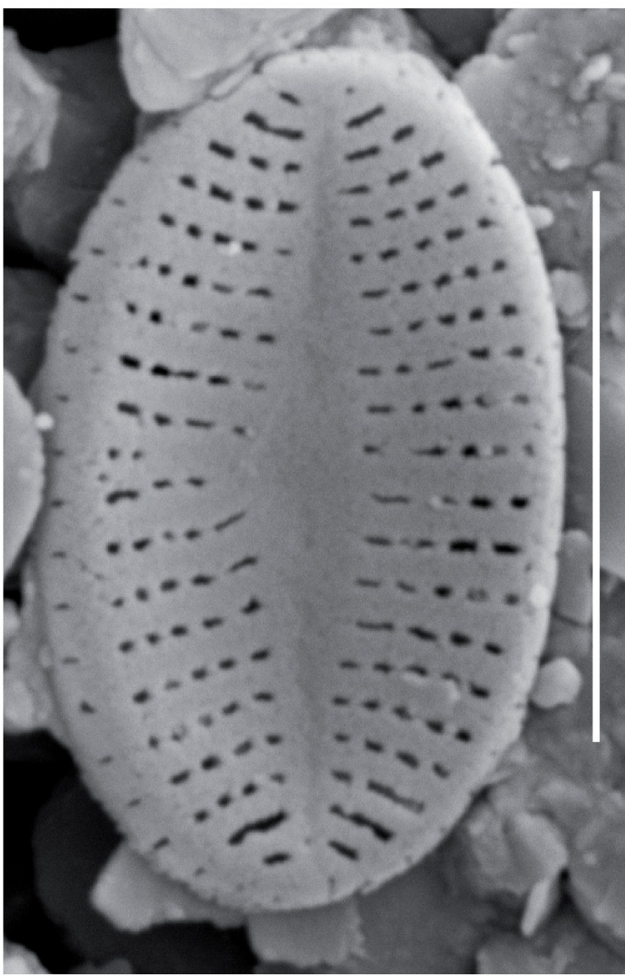

9

Plate 62: Psammothidium levanderi. - Figs 1-7: Lake Lia, LM. Figs 8-9: Lake Lia, SEM. Figs 1-2:

Raphe valve. Figs 3-7: Rapheless valves. Fig. 8: Raphe valve, outside view. Fig. 9: Rapheless valve, outside view. Scale bars $=10 \mu \mathrm{m}$ (Figs 1-7), $5 \mu \mathrm{m}$ (Figs 8-9). 
Psammotbidium marginulatum (Grunow) Bukhtiyarova et Round 1996: 5, figs $2-11$

(Plate 63: Figs 1-13)

References: Bukhtiyarova and Round (1996), Potapova (2010f), HofMANN et al. (2013).

Distribution in glacial lakes in the Retezat Mountains

\begin{tabular}{ll}
\hline Lakes & Lia, Pietrelice-1, Pietrelice-3, Stirbu, Stevia \\
Relative abundance $(\max )$. & $0.5 \%$ \\
Constancy & $2(22 \%)$ \\
\hline
\end{tabular}

Remarks: PÉTERFI (1993) reported this species from mires and running waters as Achnanthes marginulata. Not rare in our recent study. 

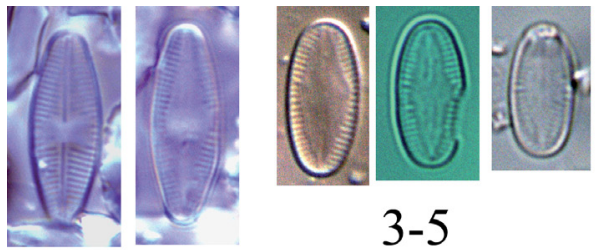

$3-5$

$1-2$

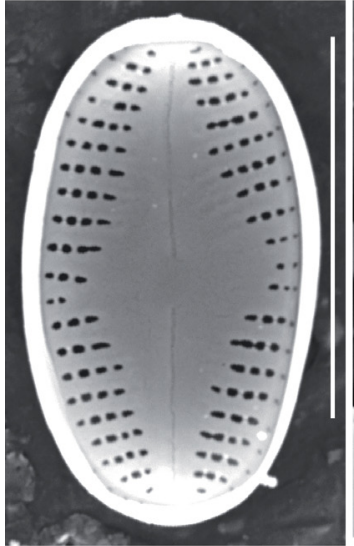

9

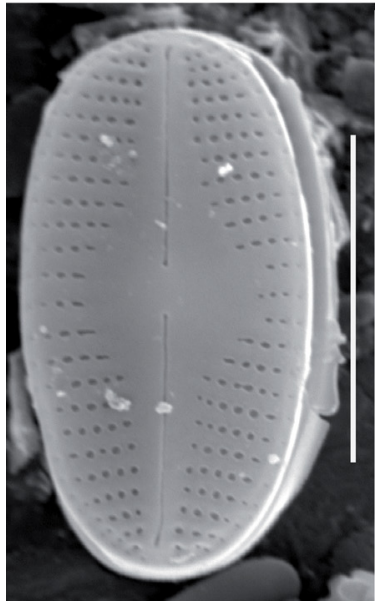

12
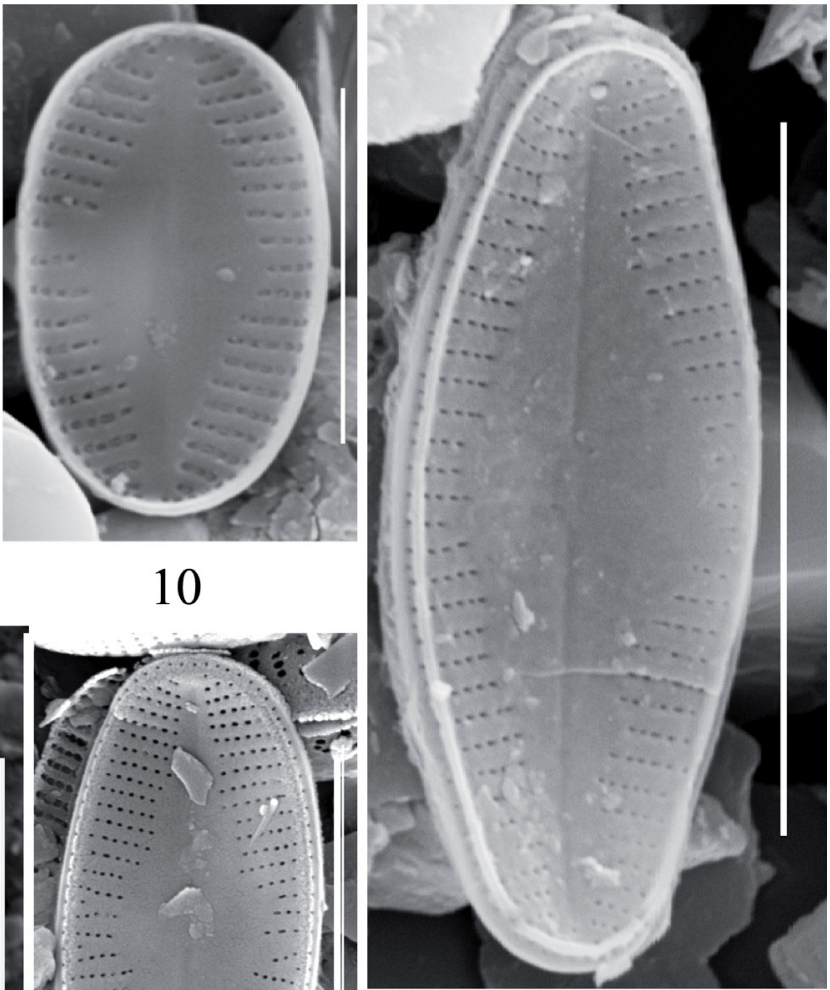

11

Plate 63: Psammothidium marginulatum. - Figs 1-2: Lake Stevia, LM. Figs 3-5: Lake Lia, LM. Figs 6-8: Lake Stevia, LM. Fig. 9: Lake Lia, raphe valve, outside view, SEM. Fig. 10: Lake Stevia, rapheless valve, SEM. Fig. 11: Lake Stevia, rapheless valve, inside view, SEM. Fig. 12: Lake Lia, raphe valve, outside view, SEM. Fig. 13: Lake Stevia, rapheless valve, outside view, SEM. Scale bars $=10 \mu \mathrm{m}$. 
Psammothidium microscopicum (Cholnoky) S. Blanco 2016: 1 (Plate 64: Figs 1-11)

References: Potapova (2010g), Blanco (2016).

Distribution in glacial lakes in the Retezat Mountains

Lakes

Ana, Bucura, Caprelor, Florica, Gales, Gemenele, Lezilor, Lia, Negru, Peleaga, Peleguta, Pietrele, Pietrelice-2, Pietrelice-3, Slavieu, Stanisoara, Stevia, Stirbu, Turcelu, Viorica, Zanoaga

Relative abundance (max.) $\quad 15.5 \%$

Constancy $5(91 \%)$

Remarks: Psammothidium curtissimum (J. R. Carter) Aboal 2003: 171 is currently regarded as a taxonomic synonym of Psammothidium microscopicum (Cholnoky) S. Blanco (GUIRY and GUIRY 2016). This taxon is very common and abundant in the glacial lakes of the Retezat Mts. 


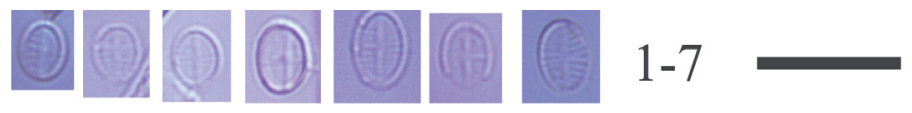

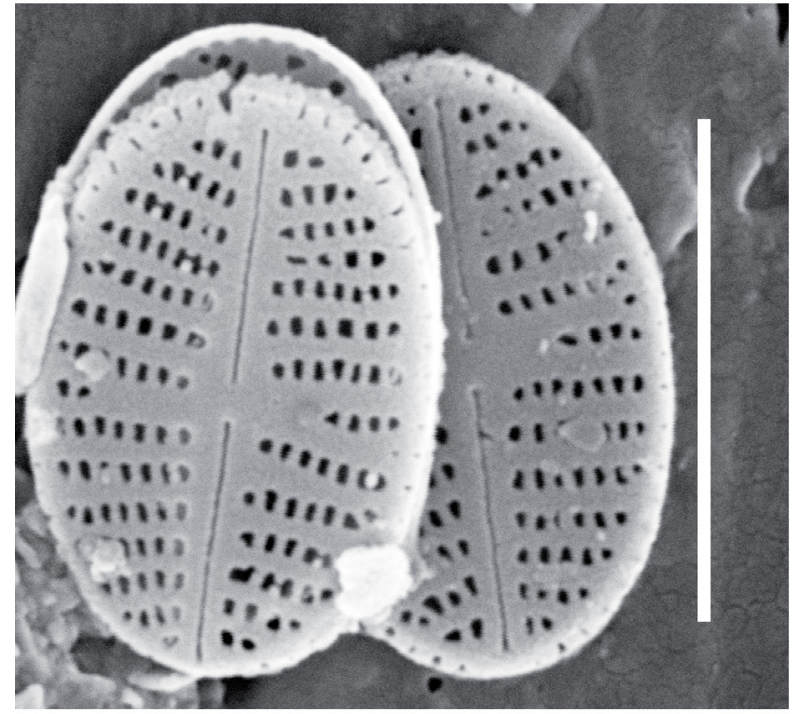

8

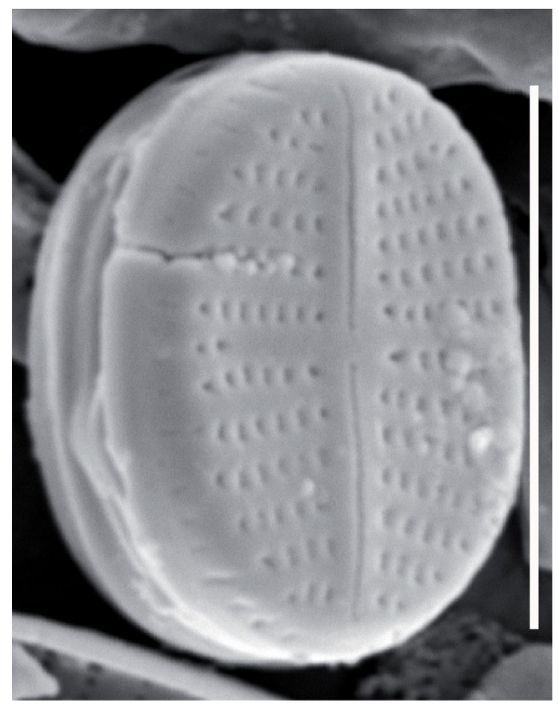

10

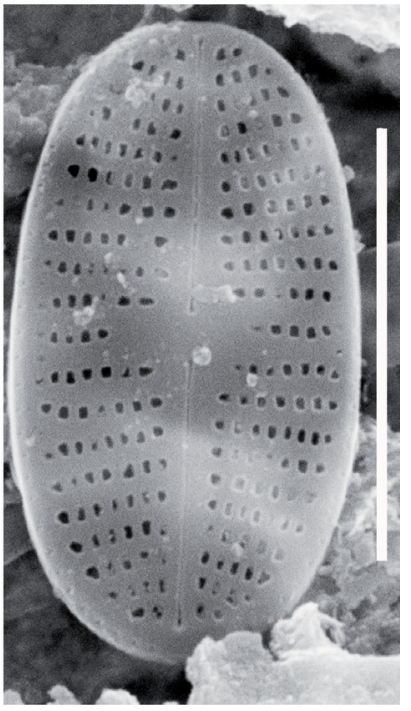

9

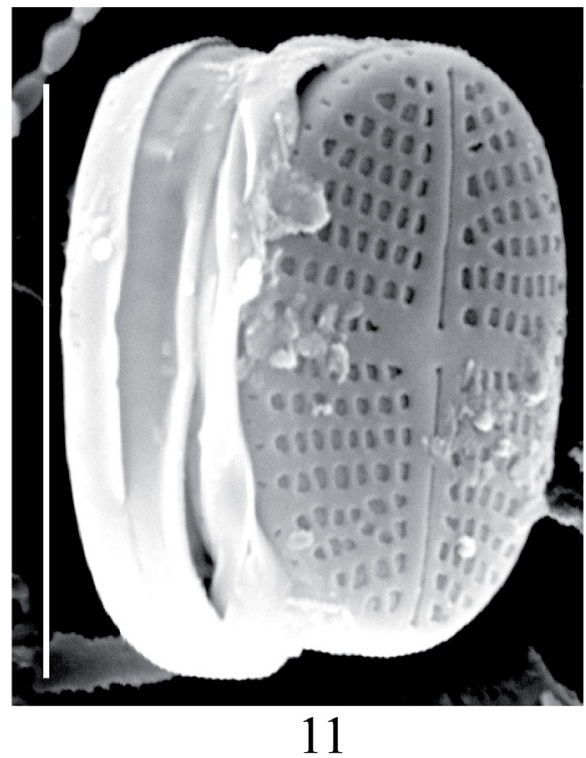

Plate 64: Psammothidium microscopicum. - Figs 1-7: Lake Pietrele, LM. Fig. 8: Lake Brazi, raphe valve, outside view, SEM. Fig. 9: Lake Brazi, raphe valve, outside view, SEM. Fig. 10: Lake Pietrele, raphe valve, outside view, SEM. Fig. 11: Lake Pietrele, raphe valve, partly girdle view. Scale bars = $10 \mu \mathrm{m}$ (Figs 1-7), $5 \mu \mathrm{m}$ (Figs 8-11). 
Psammothidium rossii (Hustedt) L. Bukhtiyarova et Round 1996:

12 , figs $40-47$

(Plate 65: Figs 1-7)

References: Bukhtiyarova and Round (1996), Hofmann et al. (2013).

Distribution in glacial lakes in the Retezat Mountains

\begin{tabular}{ll}
\hline Lakes & Lia \\
Relative abundance (max.) & $0.5 \%$ \\
Constancy & $1(4 \%)$ \\
\hline
\end{tabular}

Remark: This is a very rare species in our recent study. 


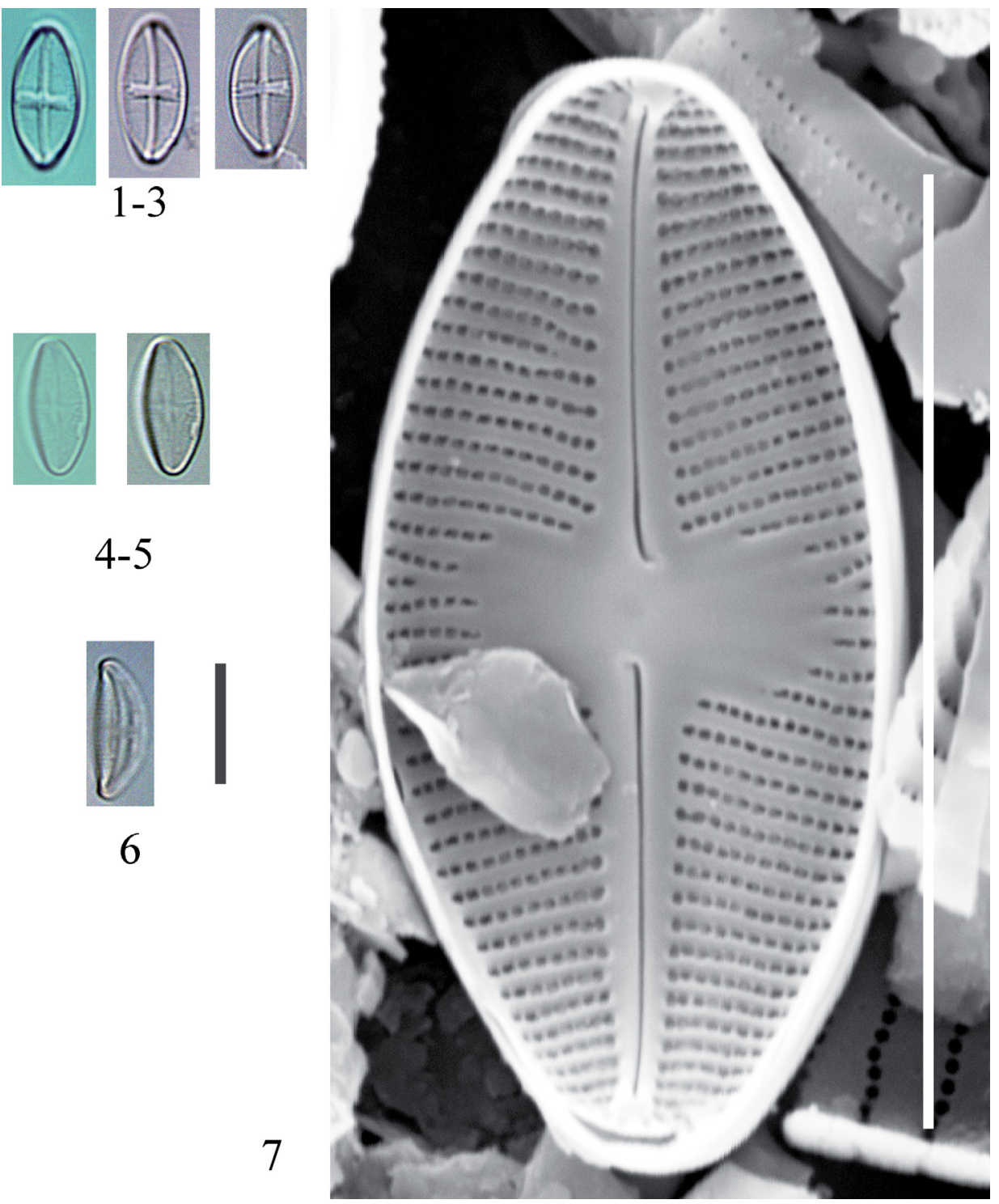

Plate 65: Psammothidium rossii. - Figs 1-3: Lake Lia, raphe valve, LM. Figs 4-5: Lake Lia, rapheless valve, LM. Fig. 6: Lake Lia, girdle view, LM. Fig. 7: Lake Lia raphe valve, inside view, SEM. Scale bars $=10 \mu \mathrm{m}$. 
Psammothidium scoticum (R. J. Flower et V. J. Jones) Bukhtiyarova et Round 1996: 22, figs 76-77

(Plate 66: Figs 1-20)

References: Flower and Jones (1989), Bukhtiyarova and Round (1996), Potapova (2010h).

Distribution in glacial lakes in the Retezat Mountains

\begin{tabular}{ll}
\hline Lakes & Ana, Brazi, Bucura, Caprelor, Florica, Gales, Gemenele, Lezilor, \\
& Lia, Peleaga, Peleguta, Pietrele, Pietrelice-1, Pietrelice-2, Pietre- \\
& lice-3, Slavieu, Stanisoara, Stevia, Viorica, Zanoaga \\
Relative abundance (max.) & $70 \%$ \\
Constancy & $5(87 \%)$ \\
\hline
\end{tabular}

Remark: Very common and very abundant diatom of the mountain lakes in the Retezat Mts. 

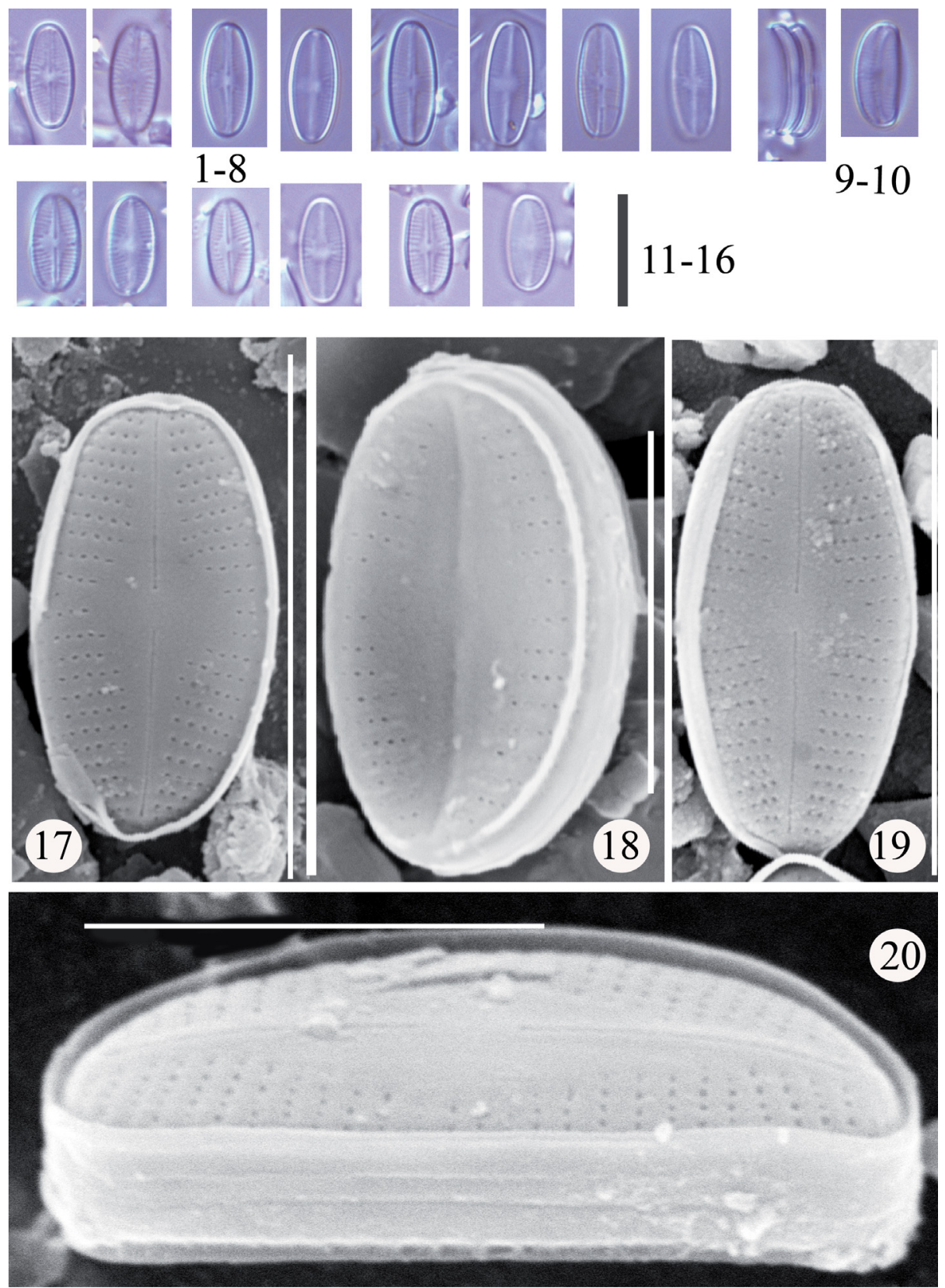

Plate 66: Psammothidium scoticum. - Figs 1-20: Lake Peleguta. Figs 1-8, 11-16: LM. Figs 9-10: Girdle view, LM. Fig. 17: Raphe valve, outside view, SEM. Fig. 18: Rapheless valve, outside view, SEM. Fig. 19: Raphe valve, outside view. Fig. 20: Raphe valve, partly girdle view, SEM. Scale bars $=10 \mu \mathrm{m}$ (Figs 1-17), $5 \mu \mathrm{m}$ (Figs 18-20). 
Psammothidium subatomoides (Hustedt) L. Bukhtiyarova et Round 1996: 13, figs 48-51

(Plate 67: Figs 1-9)

References: Bukhtiyarova and Round (1996), Potapova (2009e), HoFMANN et al. (2013).

Distribution in glacial lakes in the Retezat Mountains

\begin{tabular}{ll}
\hline Lakes & Ana, Brazi, Bucura, Caprelor, Florica, Gales, Gemenele, Lezilor, \\
& Lia, Negru, Peleaga, Peleguta, Pietrele, Pietrelice-1, Pietrelice-2, \\
& Pietrelice-3, Slavieu, Stanisoara, Stevia, Stirbu, Turcelu, Viorica, \\
& Zanoaga \\
Relative abundance (max.) & $15 \%$ \\
Constancy & $5(100 \%)$ \\
\hline
\end{tabular}

Remarks: PÉTERFI (1993) found it in lakes, and reported as Navicula subatomoides. It is one of the most common and abundant species in our recent study. 


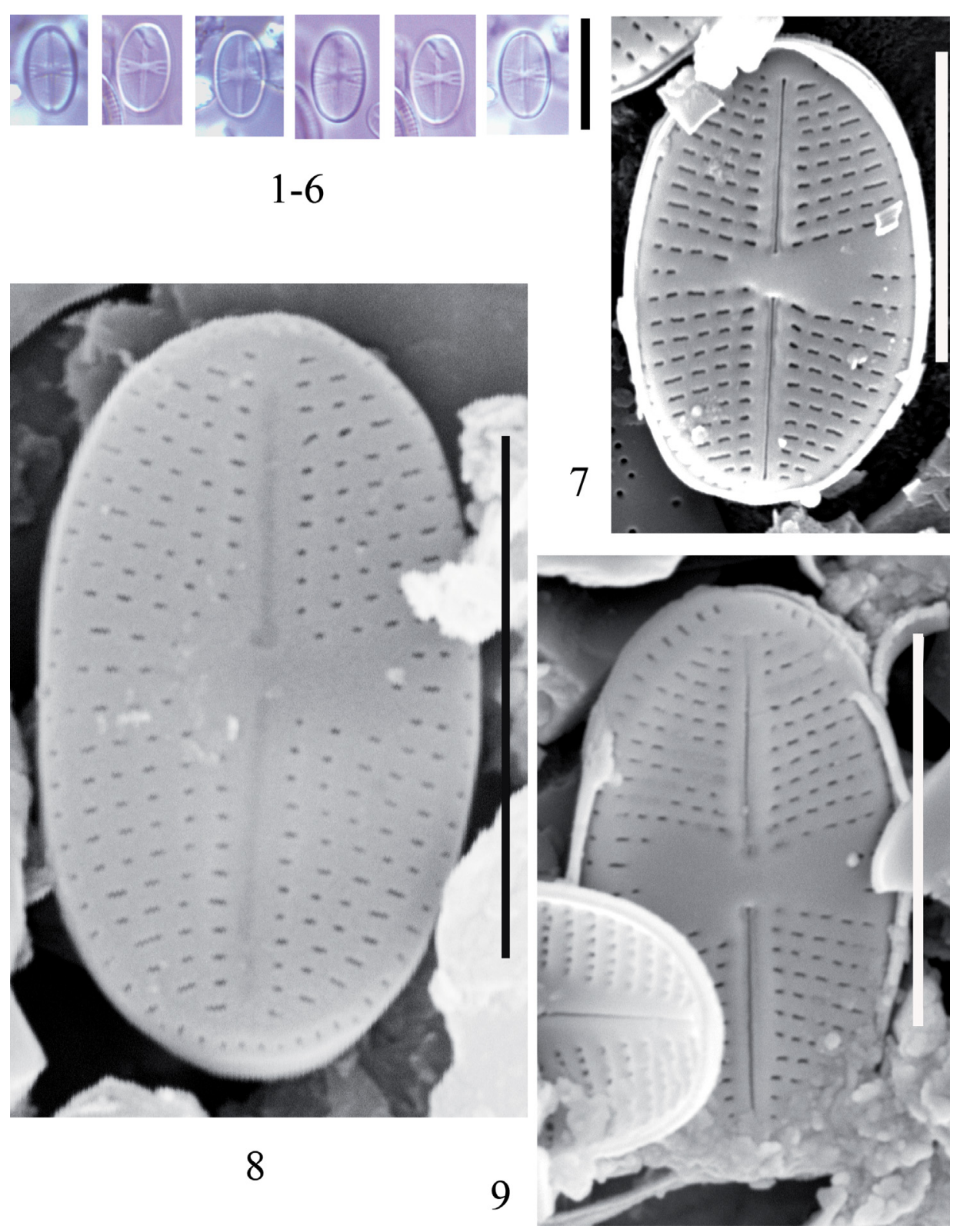

Plate 67: Psammothidium subatomoides. - Figs 1-6: Lake Stevia, LM. Fig. 7: Lake Brazi raphe valve, outside view, SEM. Fig. 8: Lake Stevia, rapheless valve, outside view, SEM. Fig. 9: Lake Stevia, raphe valve, outside view, SEM. Scale bars $=10 \mu \mathrm{m}$ (Figs 1-6), $5 \mu \mathrm{m}$ (Figs 7-9). 
Pseudostaurosira pseudoconstruens (Marciniak) D. M. Williams et Round 1988: 278

(Plate 68: Figs 1-9)

References: Williams and Round (1988a), HofmANn et al. (2013) as Staurosira pseudoconstruens, SMITH (2013).

Distribution in glacial lakes in the Retezat Mountains

\begin{tabular}{ll}
\hline Lakes & Gales \\
Relative abundance $(\max )$. & $2 \%$ \\
Constancy & $1(4 \%)$ \\
\hline
\end{tabular}

Remark: In the Retezat Mts this is a rare species. 

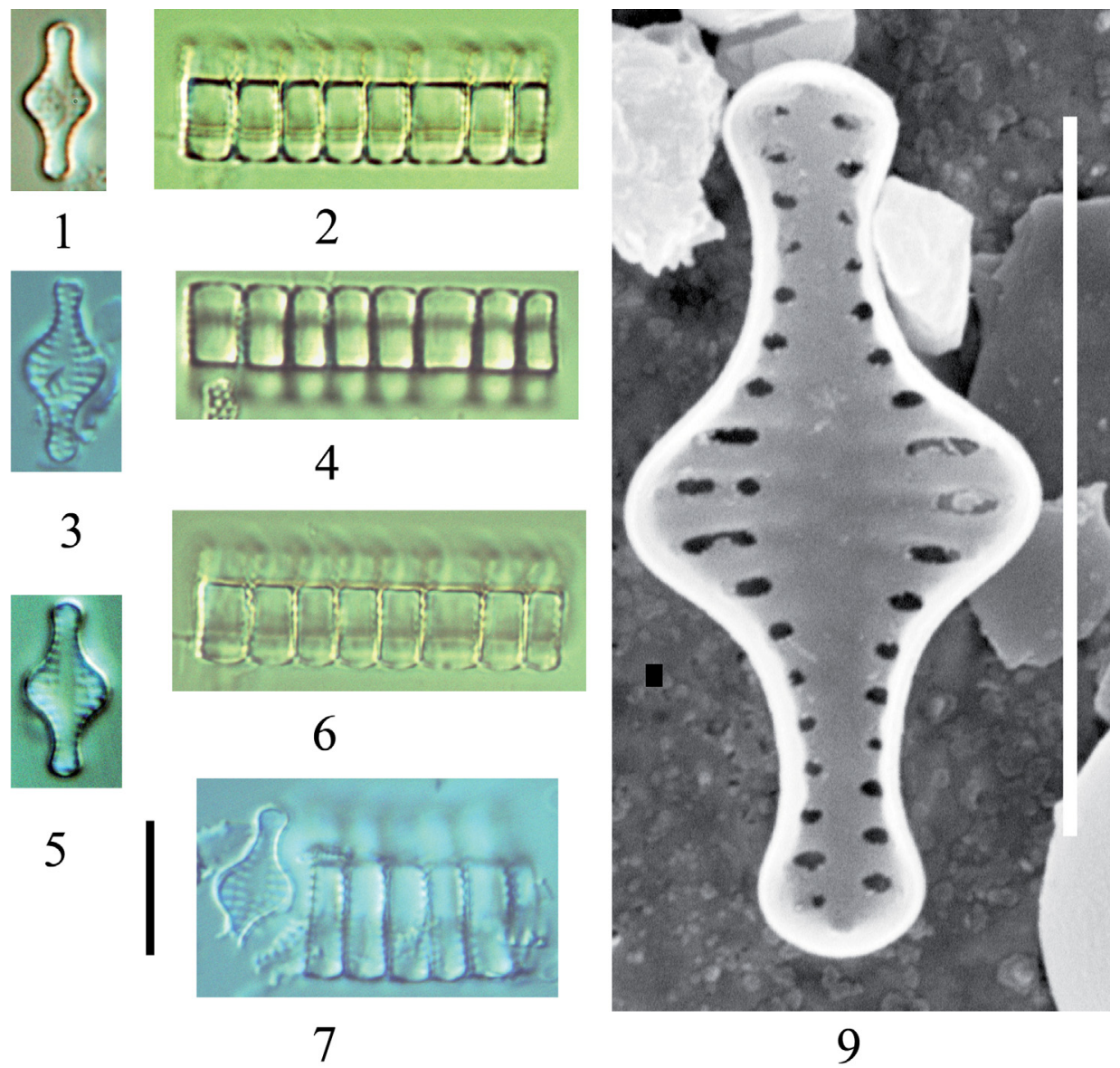

6

5

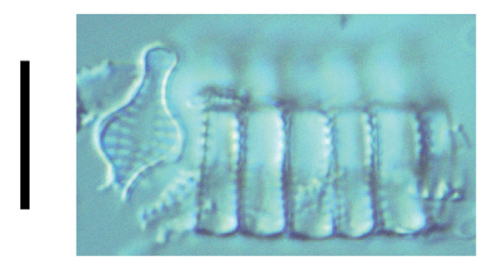

7

9

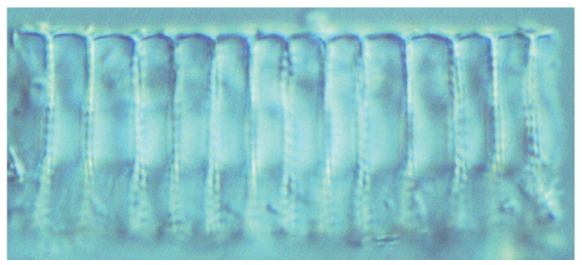

8

Plate 68: Pseudostaurosira pseudoconstruens. - Figs 1, 3, 5: Lake Gales, LM. Figs 2, 4, 6-8: Lake Gales, girdle view, LM. Fig. 9: Lake Gales, inside view, SEM. Scale bars $=10 \mu \mathrm{m}$. 
Sellaphora elorantana (H. Lange-Bertalot) C. E. Wetzel in Wetzel, C. E., Ector, L., Van de Vijver, B., Compère, P. et Mann, D. G. 2015: 226

(Plate 69: Figs 1-7)

References: Lange-Bertalot and Metzeltin (1996), Wetzel et al. (2015).

Distribution in glacial lakes in the Retezat Mountains

\begin{tabular}{ll}
\hline Lakes & Lia \\
Relative abundance $(\max )$. & $0.5 \%$ \\
Constancy & $1(4 \%)$ \\
\hline
\end{tabular}

Remark: This species was found only in one lake, rare. 


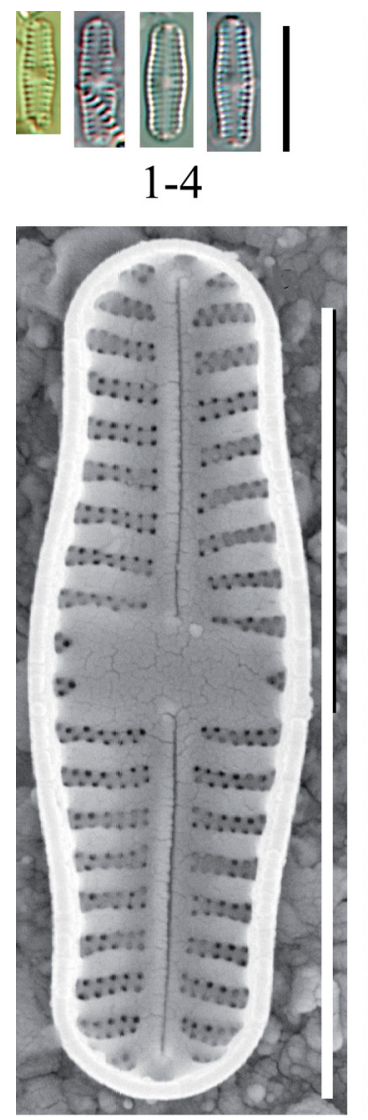

5

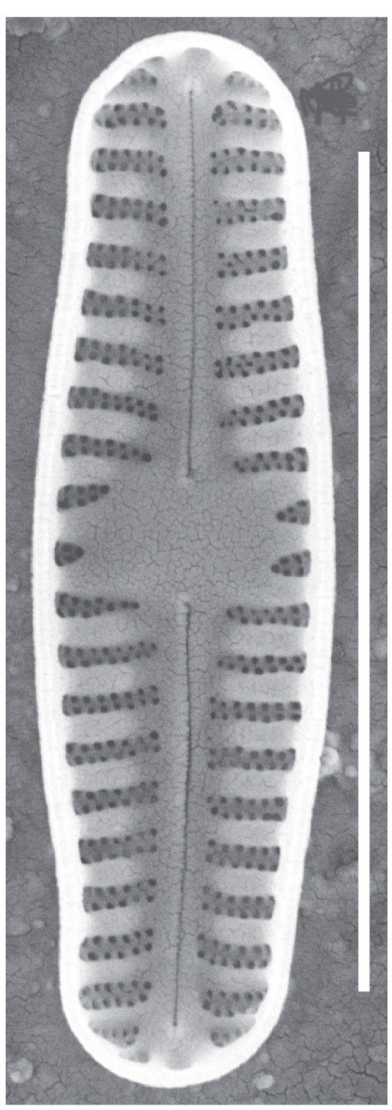

6

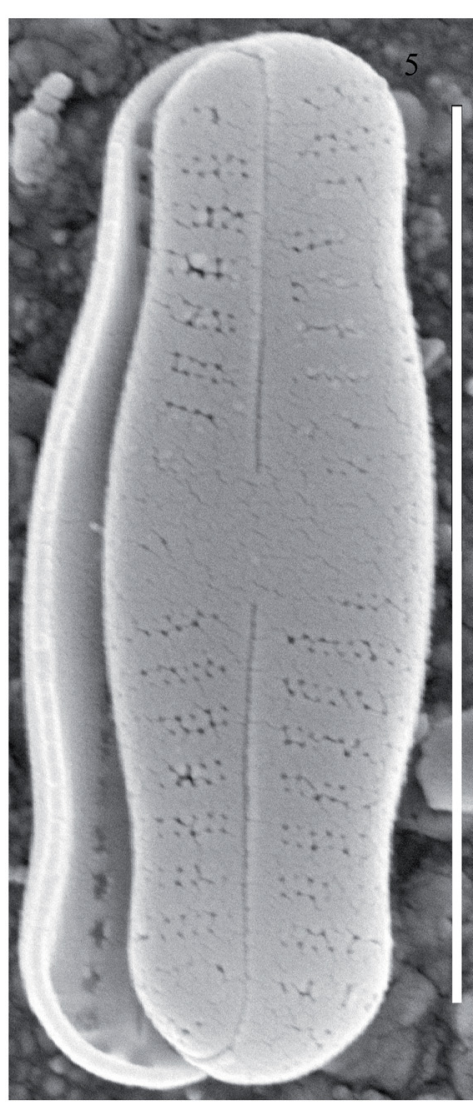

7

Plate 69: Sellaphora elorantana. - Figs 1-4: Lake Brazi, LM. Figs 5-6: Lake Brazi, inside view, SEM. Fig. 7: Lake Brazi, outside view, SEM. Scale bars $=10 \mu \mathrm{m}$. 
Sellaphora nigri (De Not.) C. E. Wetzel et L. Ector in Wetzel, C. E., Ector, L., Van de Vijver, B., Compère, P. et Mann, D. G. 2015: 227 (Plate 70: Figs 1-8)

References: Hofmann et al. (2013) as Eolimna minima, Wetzel et al. (2015).

Distribution in glacial lakes in the Retezat Mountains

\begin{tabular}{ll}
\hline Lakes & Caprelor, Gales, Lezilor, Lia, Negru, Peleguta, Stirbu, Viorica \\
Relative abundance (max.) & $1 \%$ \\
Constancy & $2(35 \%)$ \\
\hline
\end{tabular}

Remark: This species is not rare, but never abundant taxon in our recent study. 

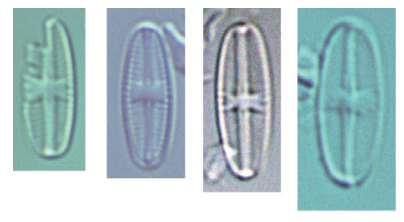

$1-4$

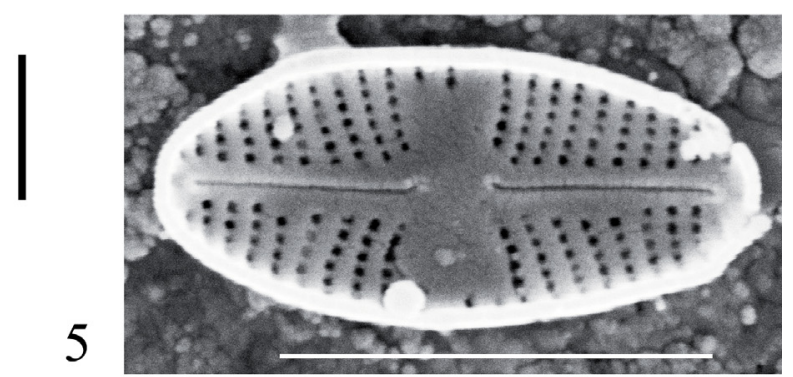

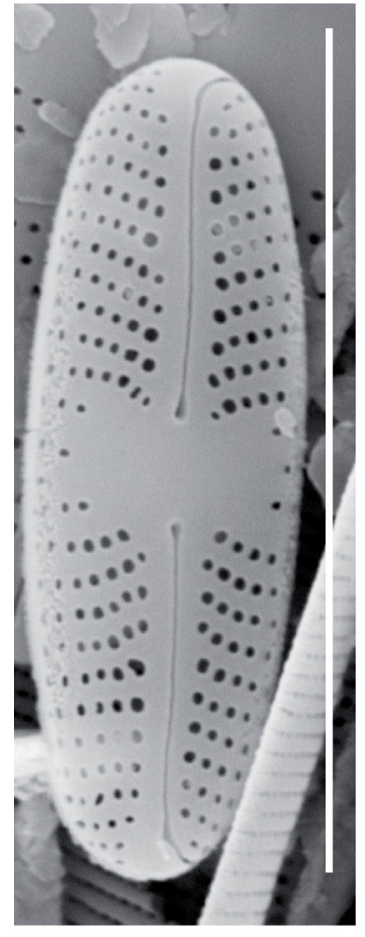

6

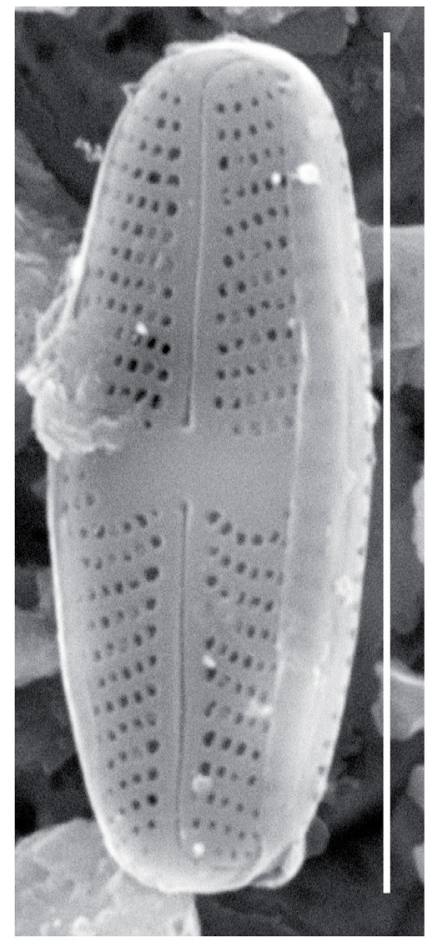

7

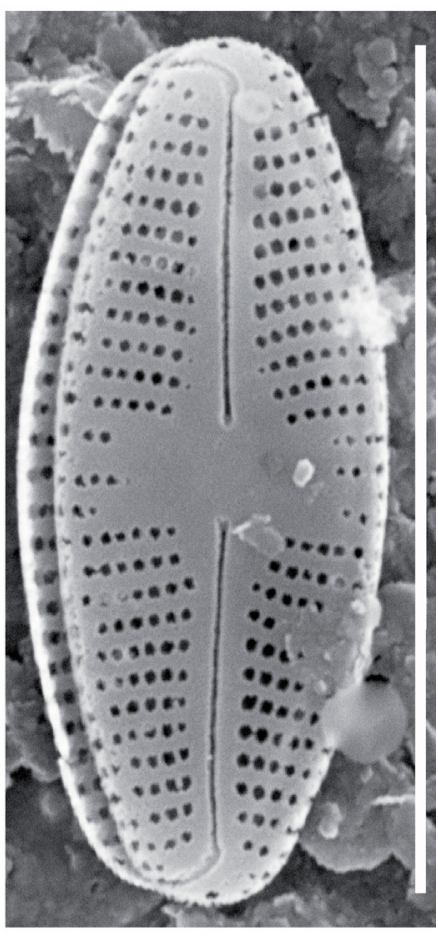

8

Plate 70: Sellaphora nigri. - Figs 1-4: Lake Lia, LM. Fig. 5: Lake Lia, inside view, SEM. Fig. 6: Lake Lia, outside view, SEM. Fig. 7: Lake Caprelor, outside view, SEM. Fig. 8: Lake Brazi, outside view, SEM. Scale bars $=10 \mu \mathrm{m}$. 
Sellaphora stauroneioides (Lange-Bertalot) J. Veselá et J. R. Johansen 2009: 461

(Plate 71: Figs 1-8)

Reference: Veselá and JohanSen (2009).

Distribution in glacial lakes in the Retezat Mountains

\begin{tabular}{ll}
\hline Lakes & Lia, Peleguta, Pietrele \\
Relative abundance (max.) & $0.5 \%$ \\
Constancy & $1(12 \%)$ \\
\hline
\end{tabular}

Remark: Rare but characteristic species in the Retezat Mts. 


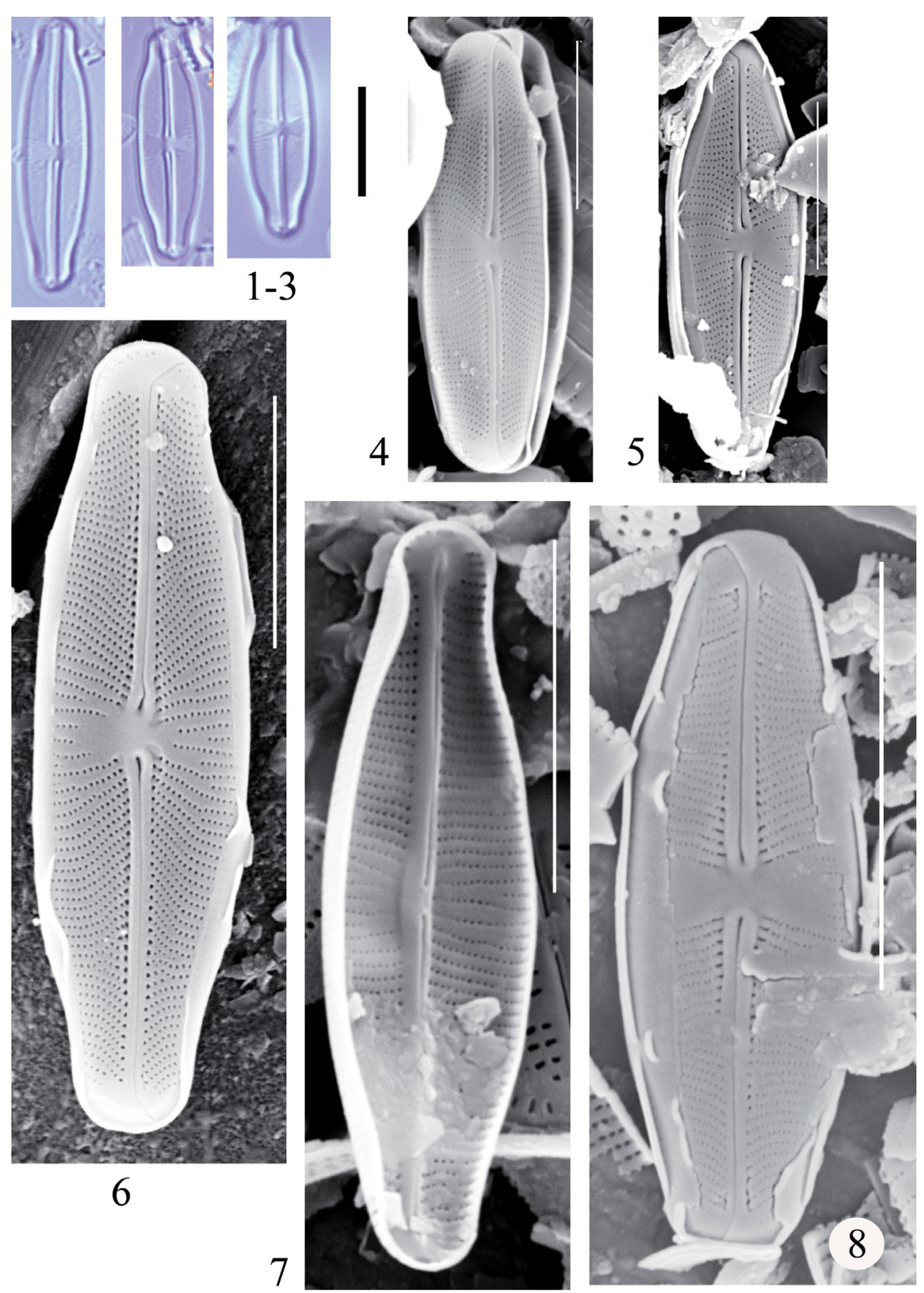

Plate 71: Sellaphora stauroneioides. - Figs 1-3: Lake Pietrele, LM. Figs 4-6: Lake Pietrele outside view, SEM. Fig. 7: Lake Peleguta, inside view, SEM. Fig. 8: Lake Pietrele, outside view, SEM. Scale bars $=10 \mu \mathrm{m}$. 
Stauroforma exiguiformis (Lange-Bertalot) R. J. Flower, V. J. Jones et F. E. Round 1996: 53

(Plate 72: Figs 1-15)

References: Flower et al. (1996), HofmANn et al. (2013), Morales and SPAULdiNg (2013).

Distribution in glacial lakes in the Retezat Mountains

\begin{tabular}{ll}
\hline Lakes & Brazi, Bucura, Caprelor, Florica, Gales, Gemenele, Lezilor, Lia, \\
& Negru, Peleaga, Pietrele, Pietrelice-2, Pietrelice-3, Slavieu, Stani- \\
& soara, Stirbu, Turcelu, Viorica, Zanoaga \\
Relative abundance (max.) & $66 \%$ \\
Constancy & $5(83 \%)$ \\
\hline
\end{tabular}

Remark: S. exiguiformis is very common and sometimes dominant species in the Retezat Mts. 

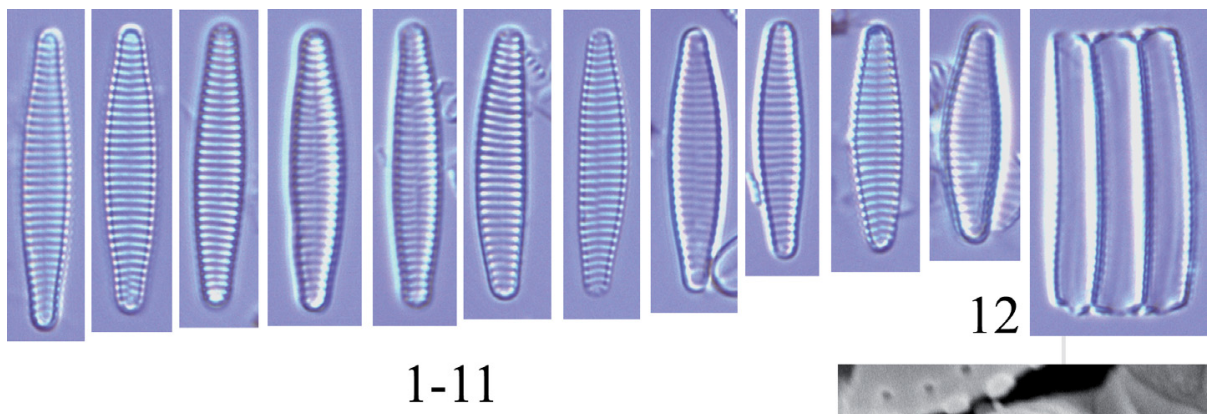

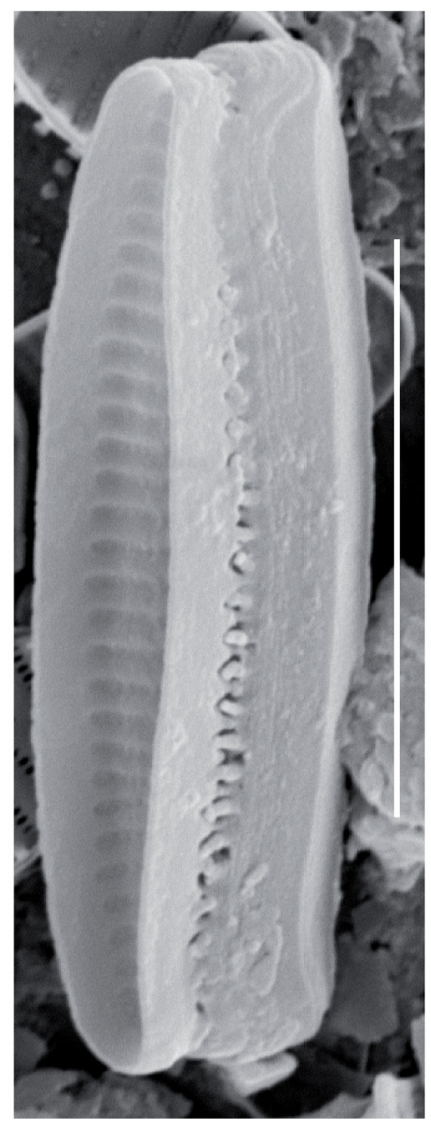

13

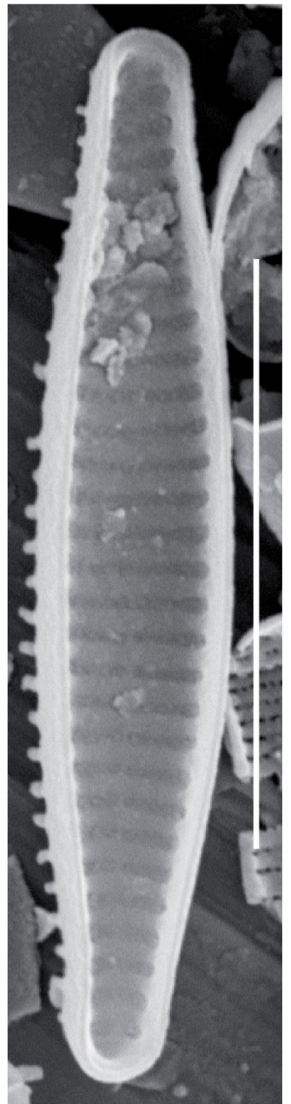

14

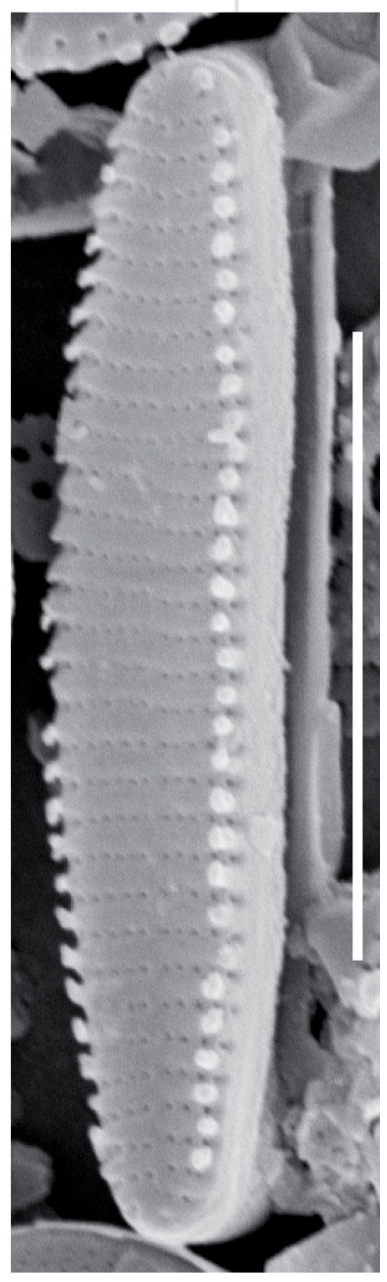

15

Plate 72: Stauroforma exiguiformis. - Figs 1-11: Lake Pietrele, LM. Fig. 12: Lake Pietrele, girdle view, LM. Fig. 13: Lake Pietrele, oblique view, SEM. Fig. 14: Lake Pietrele, inside view, SEM. Fig. 15: Lake Pietrele, outside view, SEM. Scale bars $=10 \mu \mathrm{m}$. 
Stauroneis acidoclinata Lange-Bertalot et Werum in Werum et Lange-Bertalot 2004: 173; pl. 42: figs 1-12 (Plate 73: Figs 1-9)

References: Werum and Lange-Bertalot (2004), Hofmann et al. (2013).

Distribution in glacial lakes in the Retezat Mountains

\begin{tabular}{ll}
\hline Lakes & Brazi, Bucura \\
Relative abundance (max.) & $1 \%$ \\
Constancy & $1(8 \%)$ \\
\hline
\end{tabular}

Remark: Rare species in the Retezat Mts. 

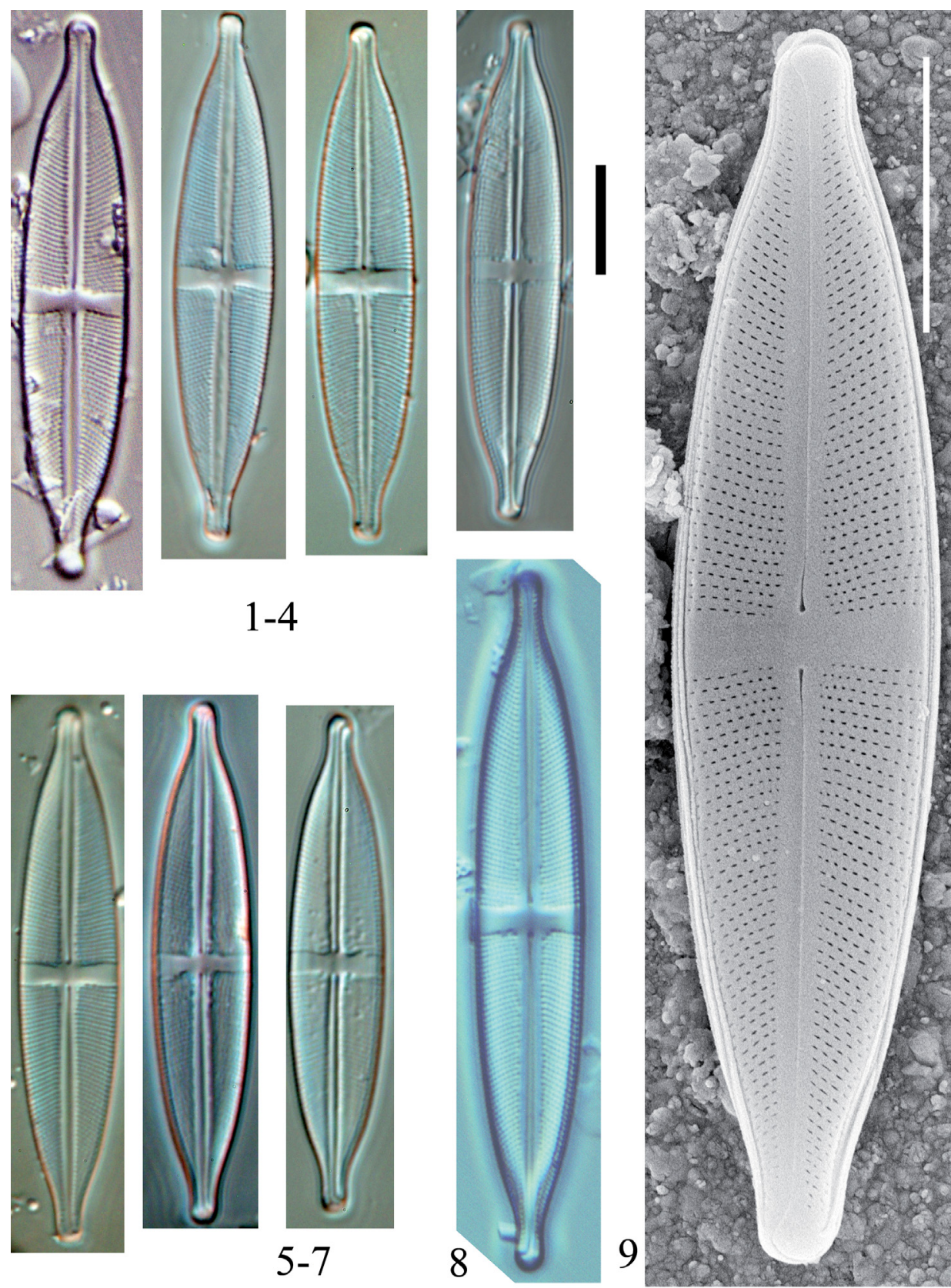

Plate 73: Stauroneis acidoclinata. - Figs 1-7: Lake Brazi, LM. Fig. 8: Lake Bucura, LM. Fig. 9: Lake Brazi, outside view, SEM. Scale bars $=10 \mu \mathrm{m}$. 
Stauroneis neofossilis Lange-Bertalot et Metzeltin 1996: 103, pl. 34: figs 4-5

(Plate 74: Figs 1-4)

Reference: LANGe-Bertalot and Metzeltin (1996).

Distribution in glacial lakes in the Retezat Mountains

\begin{tabular}{ll}
\hline Lakes & Lia \\
Relative abundance $(\max )$. & $0.5 \%$ \\
Constancy & $1(4 \%)$ \\
\hline
\end{tabular}

Remark: This is a very rare species in our recent study. There is no record on this species in AlgaeBase (GUIRY and GUIRY 2016). 

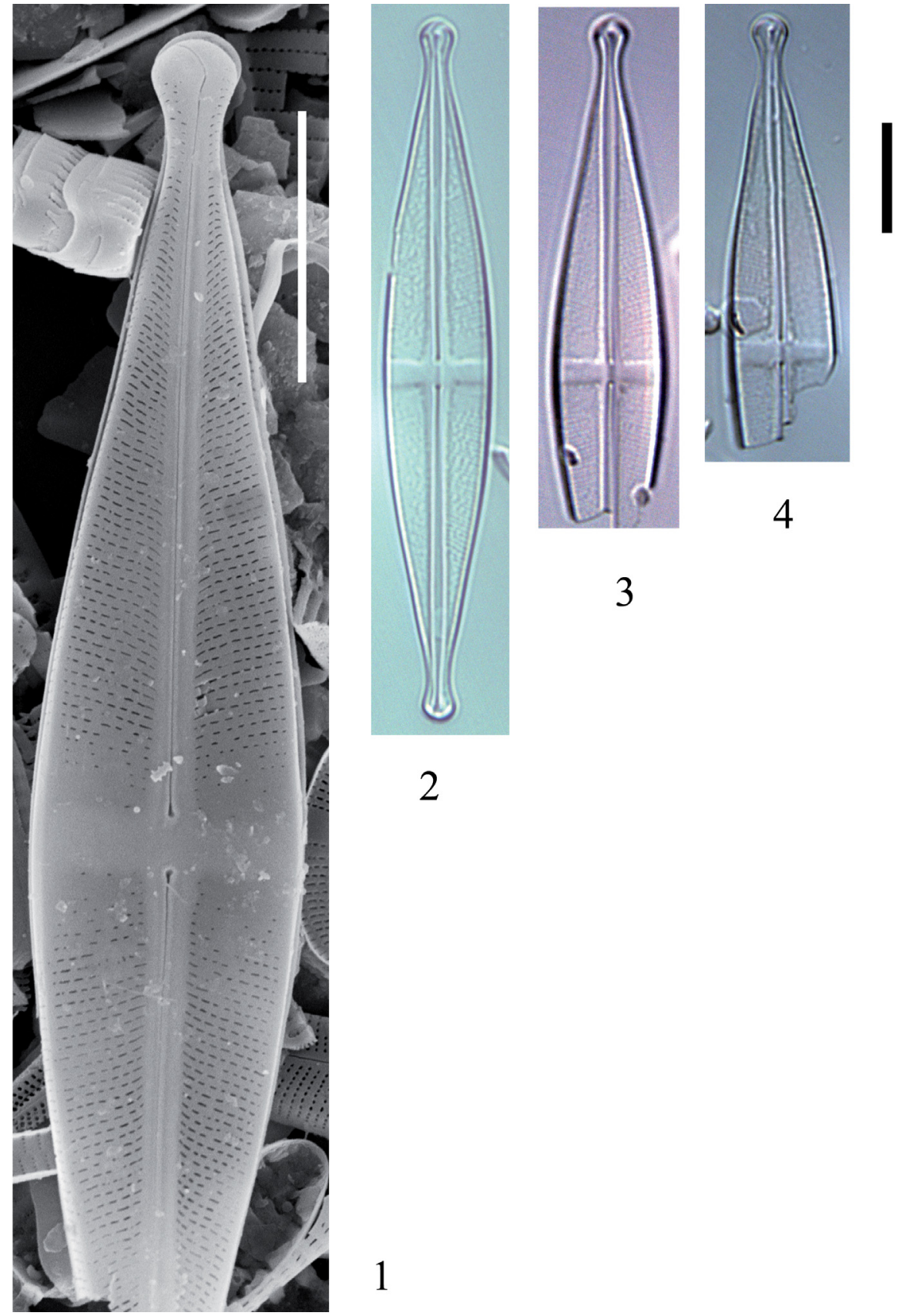

\section{3}

4
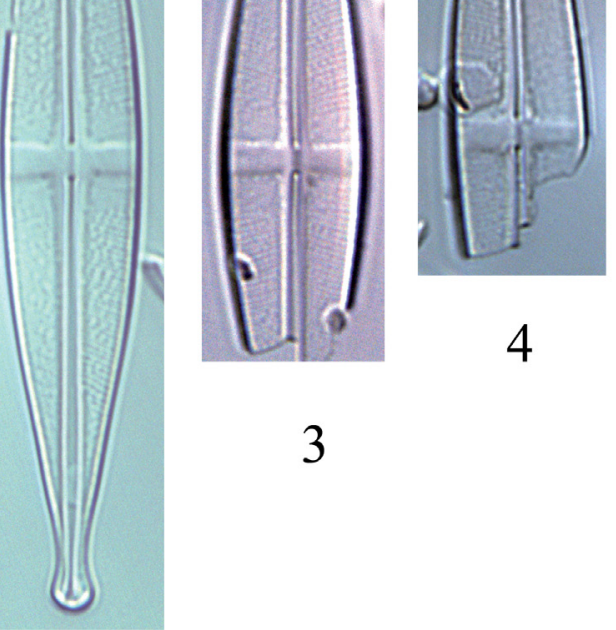

\section{2}

Plate 74: Stauroneis neofossilis. - Fig. 1: Lake Brazi, outside view, SEM. Figs 2-4: Lake Brazi, LM. Scale bars $=10 \mu \mathrm{m}$. 
Stauroneis phoenicenteron (Nitzsch) Ehrenberg 1843: 311, pl. 2/5: fig. 1, pl. 3/2: fig. 3

(Plate 75: Figs 1-4)

References: EHRENBerg (1843), Bahls (2011b), HofmanN et al. (2013).

Distribution in glacial lakes in the Retezat Mountains

\begin{tabular}{ll}
\hline Lakes & Brazi \\
Relative abundance (max.) & $0.5 \%$ \\
Constancy & $1(4 \%)$ \\
\hline
\end{tabular}

Remarks: PÉTERFI (1993) reported this species from mires, lakes and running waters. Very rare species in our recent study. 


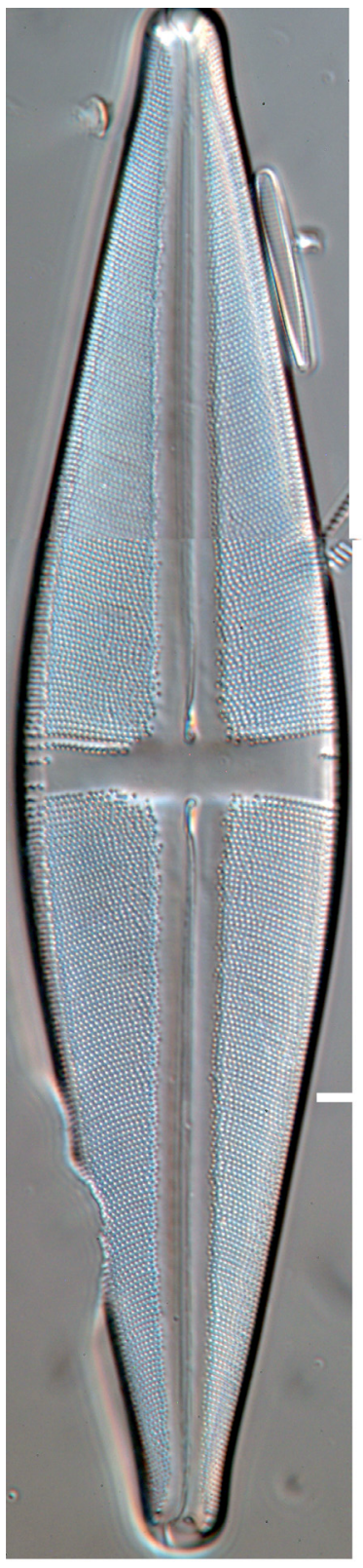

1

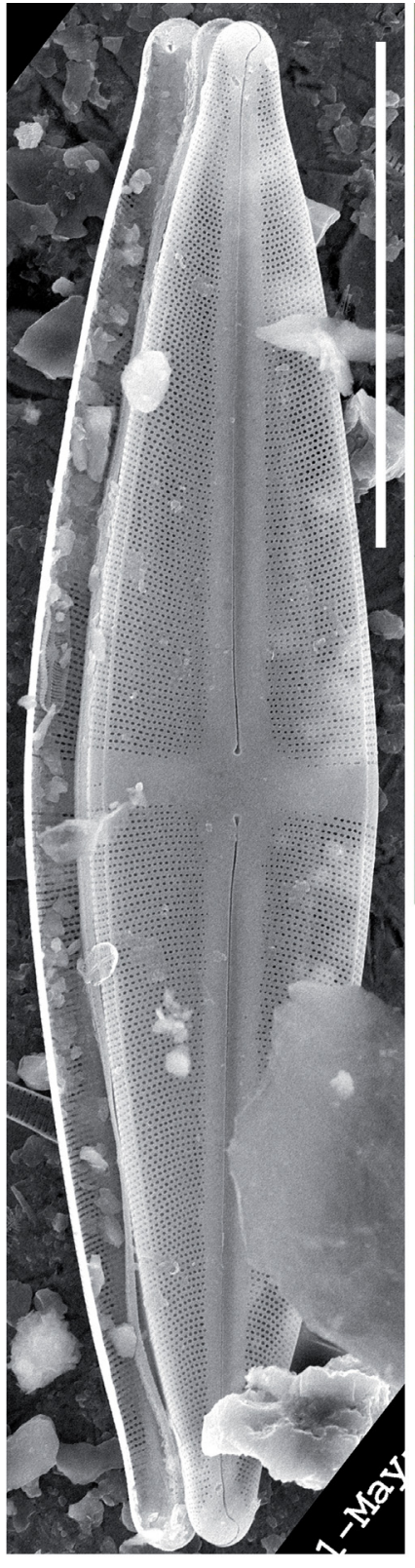

2
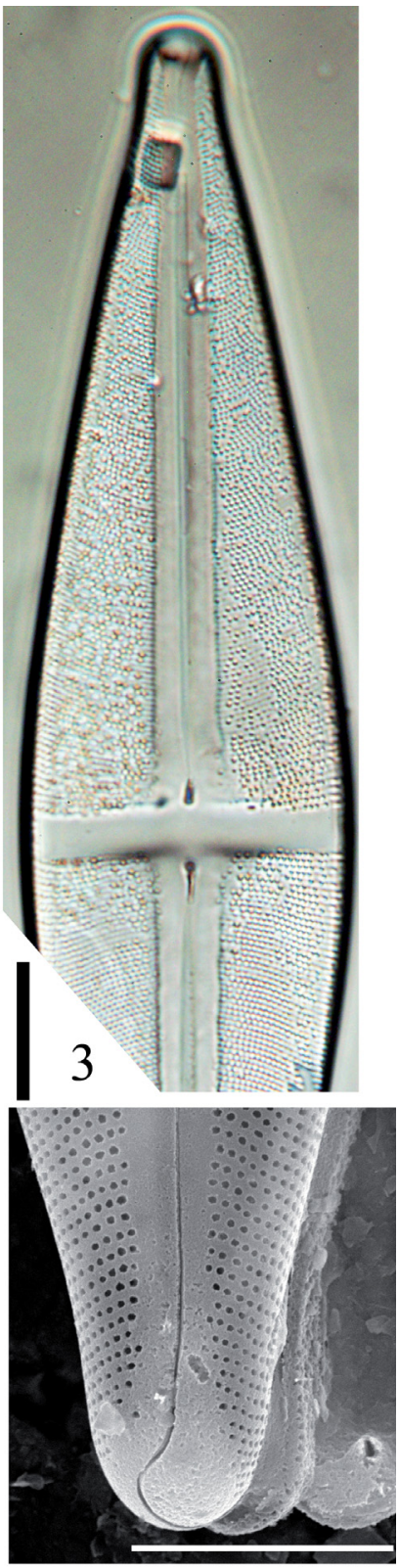

4

Plate 75: Stauroneis phoenicenteron. - Fig. 1: Lake Brazi, LM. Fig. 2: Lake Brazi, outside view, SEM. Fig. 3: Lake Brazi, LM. Fig. 4: Lake Brazi, apices, outside view, SEM. Scale bars $=10 \mu \mathrm{m}$ (Figs 1, 3-4), $50 \mu \mathrm{m}$ (Fig. 2). 
Staurosira venter (Ehrenberg) Cleve and J. D. Möller 1879: no. 242 (Plate 76: Figs 1-15)

References: Cleve and Möller (1879), Morales (2010a), Hofmann et al. (2013).

Distribution in glacial lakes in the Retezat Mountains

\begin{tabular}{ll}
\hline Lakes & $\begin{array}{l}\text { Ana, Brazi, Bucura, Caprelor, Florica, Gales, Gemenele, Lezilor, } \\
\text { Lia, Peleaga, Pietrele, Pietrelice-2, Pietrelice-3, Slavieu, Staniso- } \\
\text { ara, Stirbu, Turcelu, Viorica, Zanoaga }\end{array}$ \\
Relative abundance (max.) & $32.5 \%$ \\
Constancy & $5(83 \%)$ \\
\hline
\end{tabular}

Remark: Common and abundant species in the Retezat Mts. 

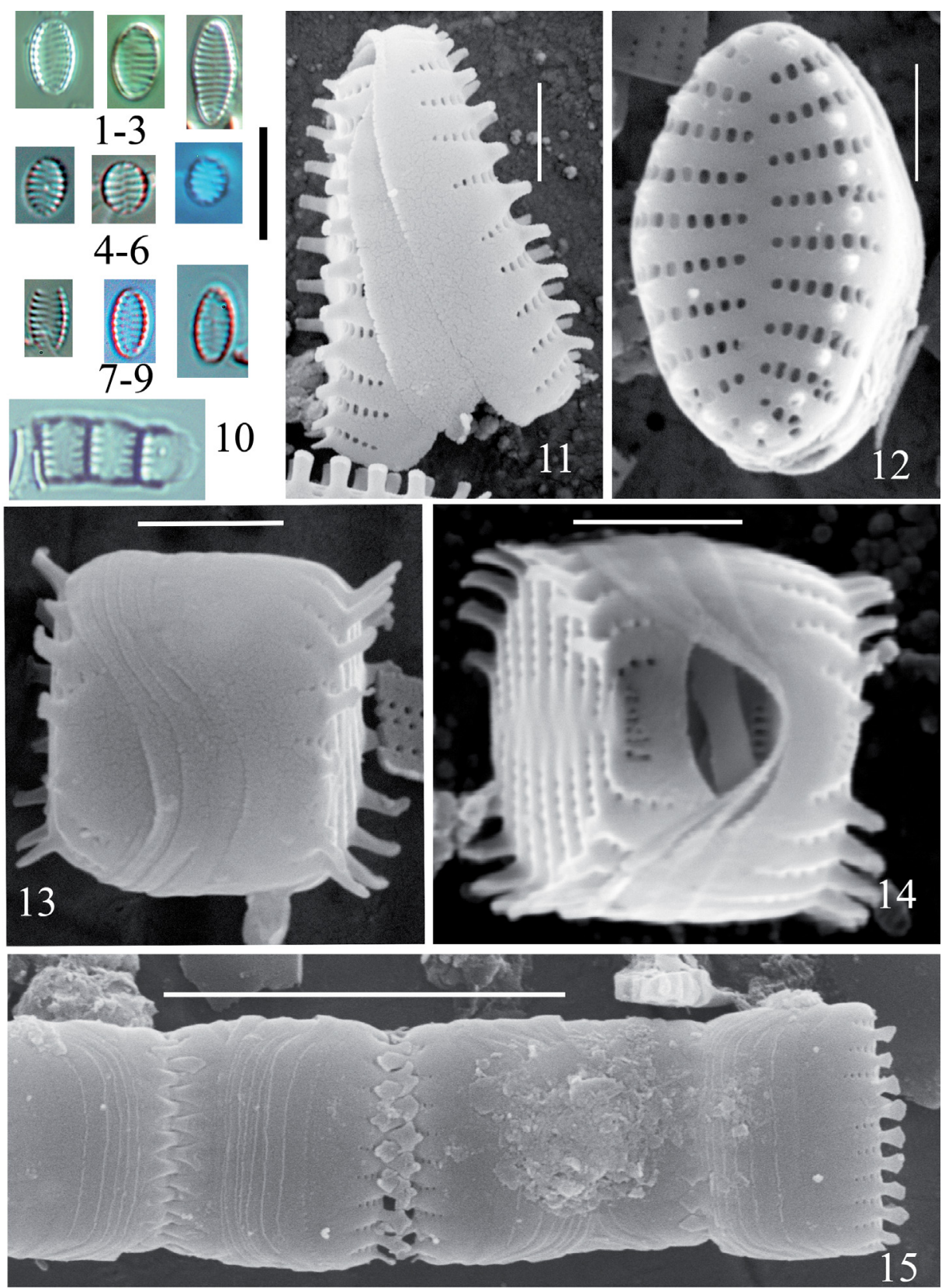

Plate 76: Staurosira venter. - Figs 1-3: Lake Peleguta, LM. Figs 4-6: Lake Brazi, LM. Figs 7-9: Lake Gales, LM. Fig. 10: Lake Peleguta, girdle view, LM. Figs 11: Lake Brazi, girdle view, SEM. Fig. 12: Lake Brazi, SEM. Figs 13-15: Lake Peleguta, girdle view, SEM. Scale bars $=10 \mu \mathrm{m}$ (Figs $1-10,15), 2 \mu \mathrm{m}$ (Figs 11-14). 
Staurosirella pinnata (Ehrenberg) D. M. Williams et Round 1988: 274

(Plate 77: Figs 1-15)

References: Williams and Round (1988a), Morales (2010b), HoFMANN et al. (2013) as Staurosira pinnata.

Distribution in glacial lakes in the Retezat Mountains

\begin{tabular}{ll}
\hline Lakes & Lezilor, Peleaga, Peleguta, Stirbu, Turcelu \\
Relative abundance (max.) & $4.5 \%$ \\
Constancy & $2(22 \%)$ \\
\hline
\end{tabular}

Remarks: PÉTERFI (1993) reported this species from lakes and running waters as Fragilaria pinnata. This is not a rare species in the Retezat Mts. 

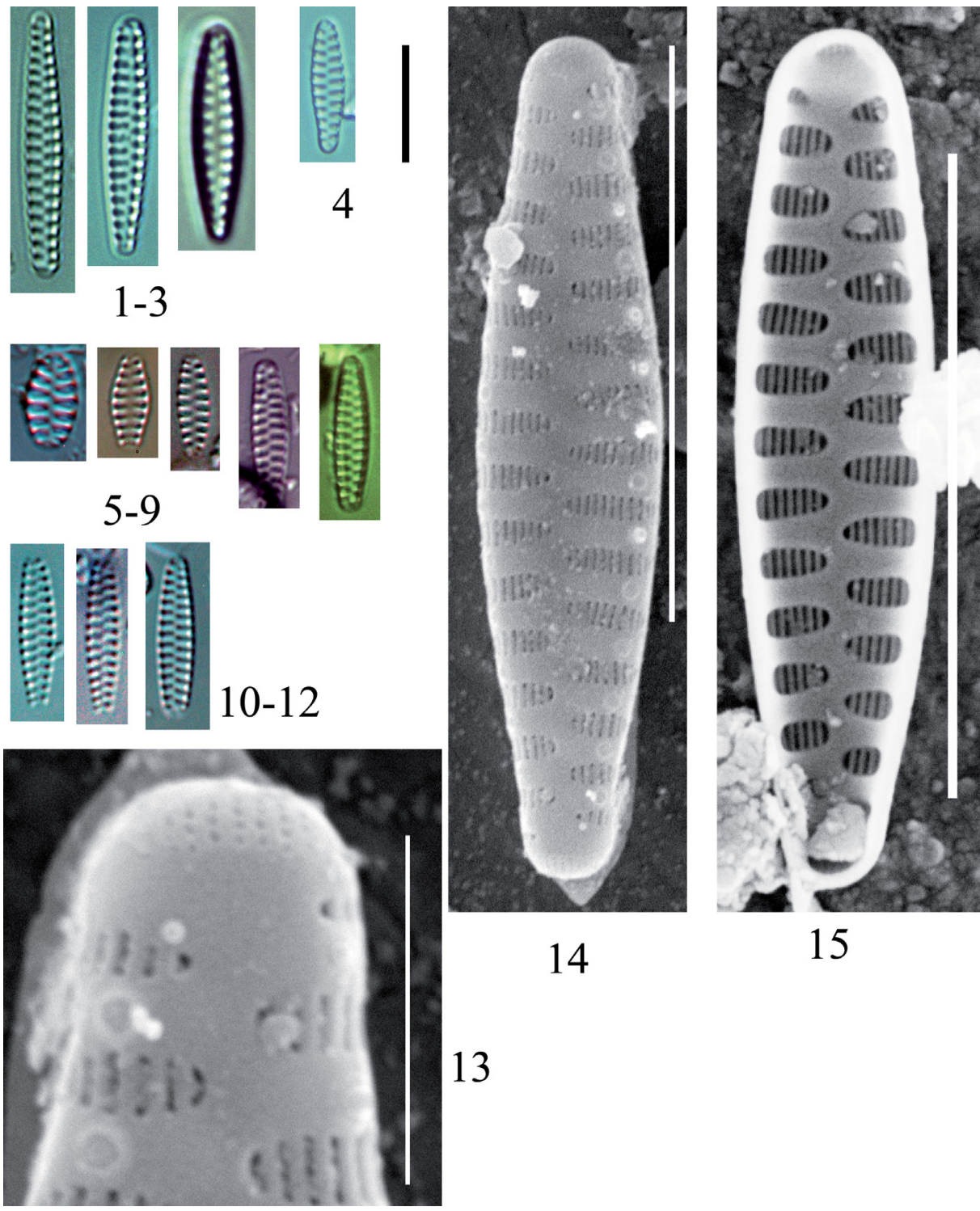

13

Plate 77: Staurosirella pinnata s. 1. - Figs 1-3: Lake Lia, LM. Fig. 4: Lake Peleguta, LM. Figs 5-9: Lake Gales, LM. Figs 10-12: Lake Brazi, LM. Fig. 13: Lake Peleguta, details of apice, outside view, SEM. Fig. 14: Lake Peleguta, outside view, SEM. Fig. 15: Lake Peleguta, outside view, SEM. Scale bars $=10 \mu \mathrm{m}$. 
Staurosirella sp.

(Plate 78: Figs 1-13)

Distribution in glacial lakes in the Retezat Mountains

\begin{tabular}{ll}
\hline Lakes & Gales \\
Relative abundance $(\max )$. & $0.5 \%$ \\
Constancy & $1(4 \%)$ \\
\hline
\end{tabular}

Remark: This species probably new for science. 

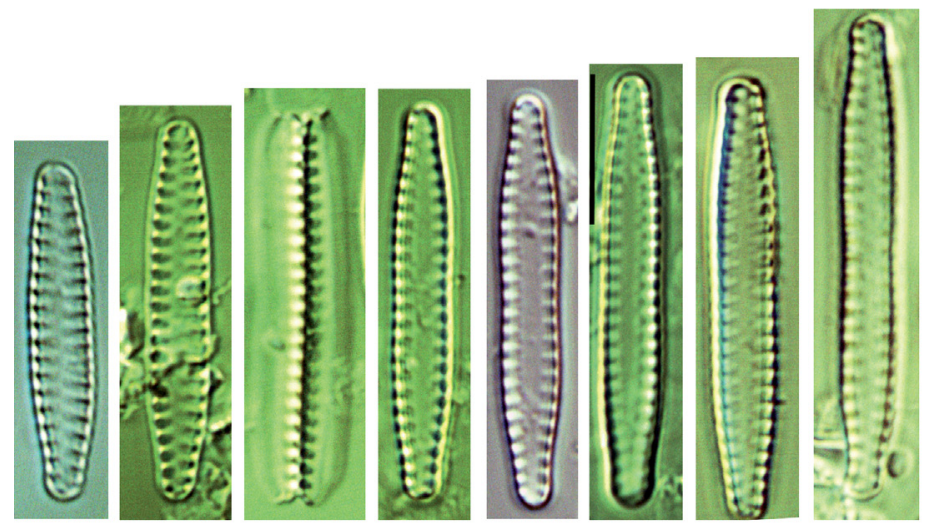

$1-8$
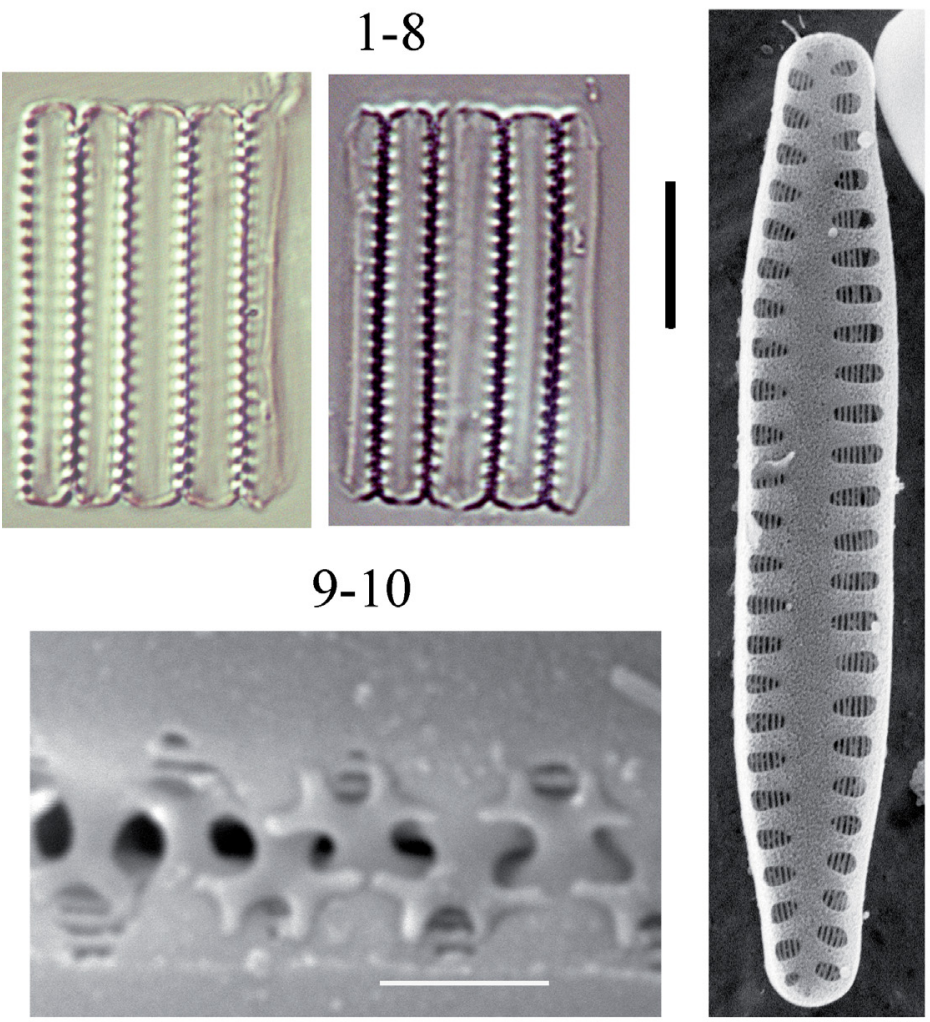

12

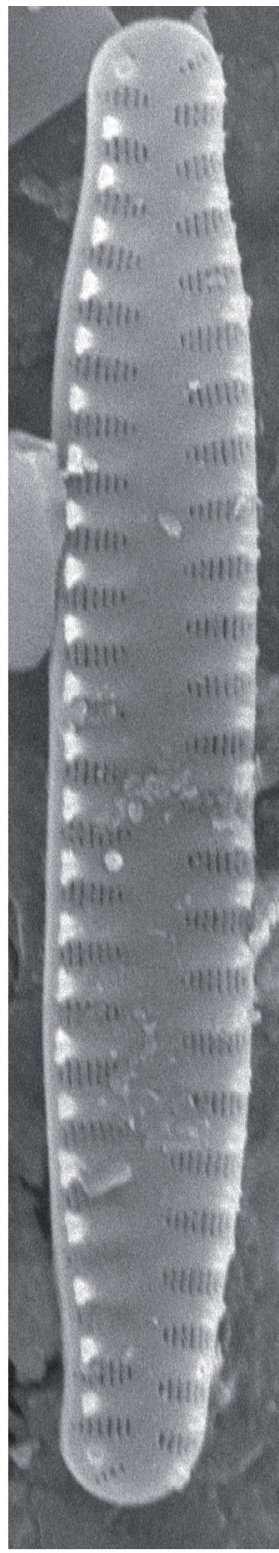

11

13

Plate 78: Staurosirella sp. - Figs 1-8: Lake Gales, LM. Figs 9-10: Lake Gales, girdle view, LM. Fig. 11: Lake Gales, spines details, girdle view, SEM. Fig. 12: Lake Gales, inside view, SEM. Fig. 13: Lake Gales, outside view, SEM. Scale bars $=10 \mu \mathrm{m}$ (Figs 1-10), $2 \mu \mathrm{m}$ (Figs 12-13). 
Stenopterobia delicatissima (F. W. Lewis) Brébisson ex Van Heurck 1896: 374, figs 19-51

(Plate 79: Figs 1-13)

References: VAN Heurck (1896), HofmanN et al. (2013).

Distribution in glacial lakes in the Retezat Mountains

Lakes Bucura, Florica, Gemenele, Lia, Negru, Pietrele, Pietrelice-1, Stavieu, Stanisoara, Viorica

Relative abundance (max.) $\quad 1.7 \%$

Constancy $3(44 \%)$

Remark: Common, but never abundant species in our recent study. 

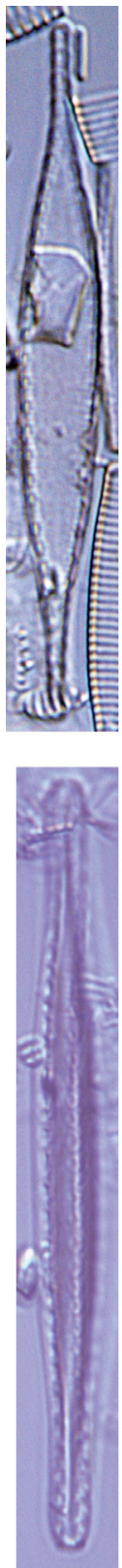
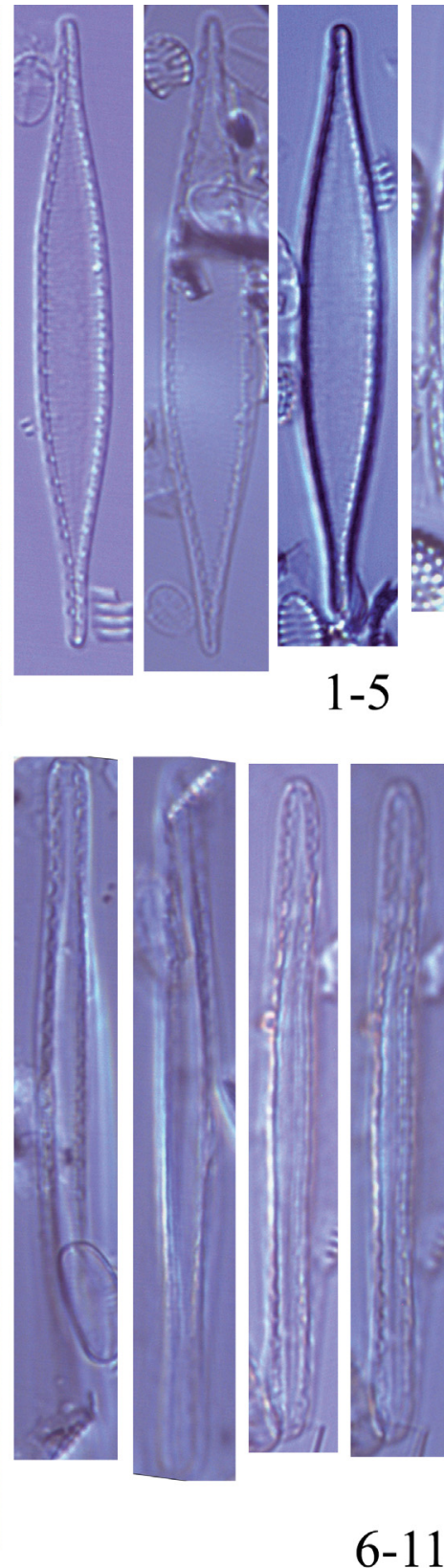

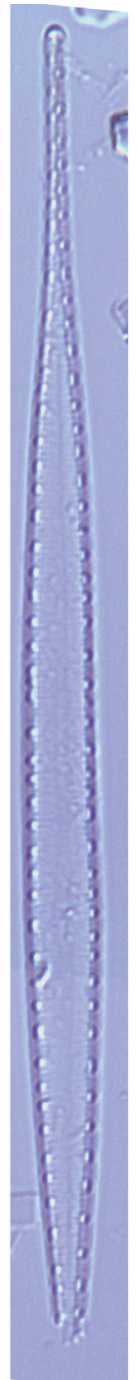

12

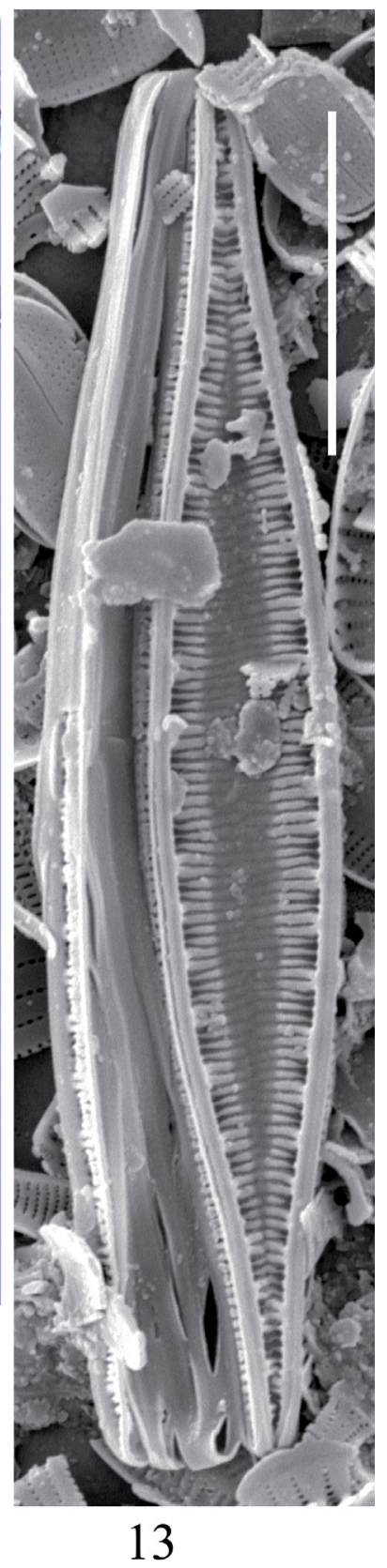

Plate 79: Stenopterobia delicatissima. - Figs 1-5: Lake Pietrele, LM. Figs 6-11: Lake Pietrele girdle view, LM. Fig. 12: Lake Zaonaga, LM. Fig. 13: Lake Pietrele, outside view, SEM. Scale bars $=10 \mu \mathrm{m}$. 
Surirella angusta Kützing 1844: 61, pl. 30: fig. 52

(Plate 80: Figs 1-4)

References: KÜtZING (1844), HofmANn et al. (2013).

Distribution in glacial lakes in the Retezat Mountains

\begin{tabular}{ll}
\hline Lakes & Lia \\
Relative abundance (max.) & $0.5 \%$ \\
Constancy & $1(4 \%)$ \\
\hline
\end{tabular}

Remark: This is rare species in our recent study.

Surirella bifrons Ehrenberg 1843: 388, pl. 3: fig. 5, pl. 4: fig. 1

(Plate 80: Fig. 5)

References: EHRENBERg (1843), HofmANn et al. (2013).

Distribution in glacial lakes in the Retezat Mountains

\begin{tabular}{ll}
\hline Lakes & Lia \\
Relative abundance (max.) & $0.5 \%$ \\
Constancy & $1(4 \%)$ \\
\hline
\end{tabular}

Remarks: PÉTERfi (1993) reported this species from glacial lakes as Surirella biseriata var. bifrons. Very rare species.

Surirella tenera W. Gregory 1856: 11, pl. 1: fig. 38 (Plate 80: Fig. 6)

References: GREgory (1856), HofmAnN et al. (2013).

Distribution in glacial lakes in the Retezat Mountains

\begin{tabular}{ll}
\hline Lakes & Peleguta \\
Relative abundance (max.) & $0.5 \%$ \\
Constancy & $1(4 \%)$ \\
\hline
\end{tabular}

Remark: Rare taxon in our recent study. 

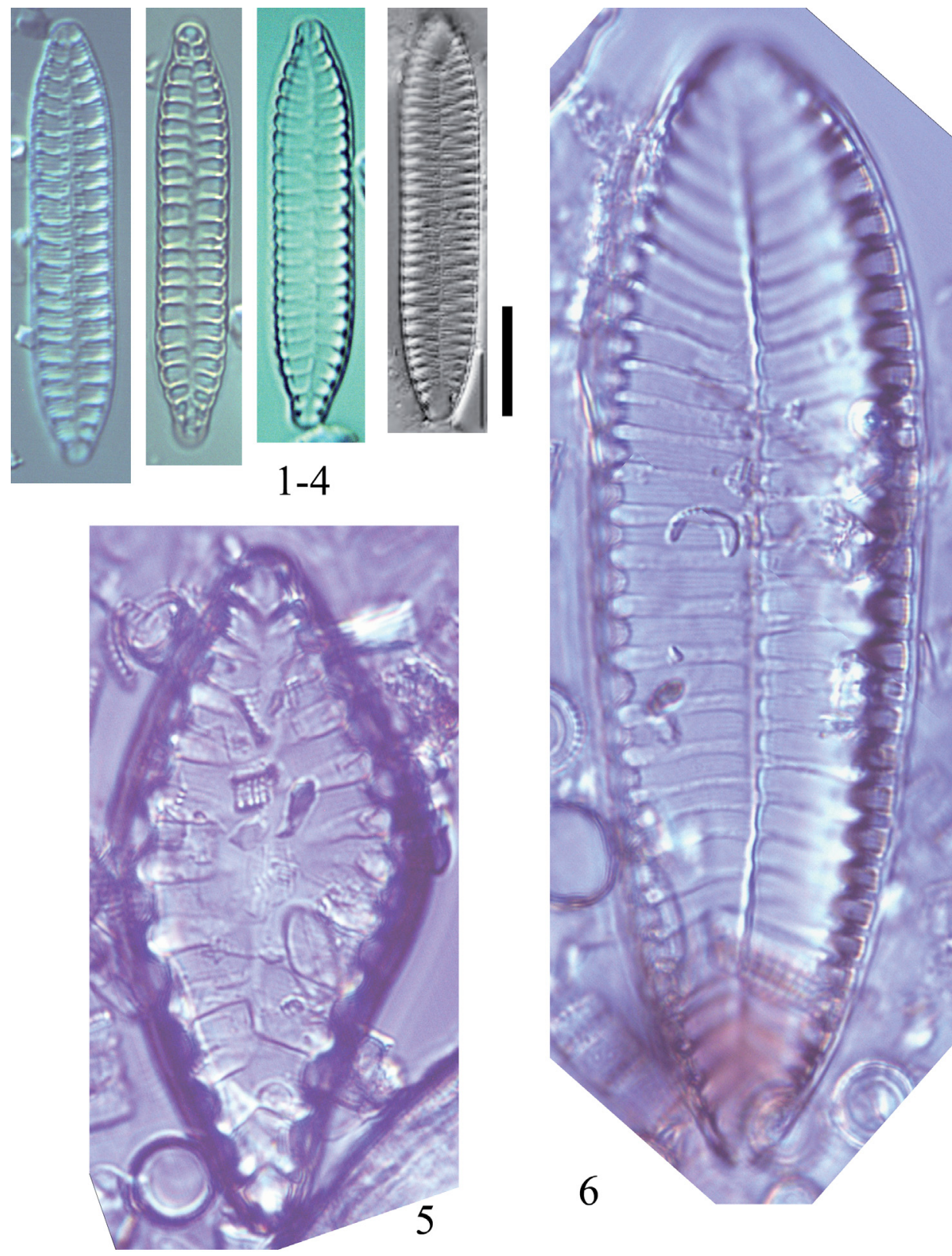

Plate 80: Surirella angusta, Surirella bifrons, Surirella tenera - Figs 1-4: Surirella angusta, Lake Lia, LM. Fig. 5: Surirella bifrons, Lake Peleguta, LM. Fig. 6: Surirella tenera, Lake Peleguta, LM. Scale bars $=10 \mu \mathrm{m}$. 
Surirella linearis W. Smith 1853: 31, pl. 8: fig. 58

(Plate 81: Figs 1-8)

References: SMith (1853), Hofmann et al. (2013).

Distribution in glacial lakes in the Retezat Mountains

Lakes

Ana, Bucura, Florica, Gales, Gemenele, Lezilor, Lia, Negru, Pele-

aga, Pietrele, Pietrelice-2, Slavieu, Stanisoara, Stevia, Stirbu

Relative abundance ( $\max ) \quad 4.5 \$.

Constancy

$4(65 \%)$

Remarks: PÉTERFI (1993) reported this species from mires, lakes and also from running waters. This is a very common species in our recent study. 

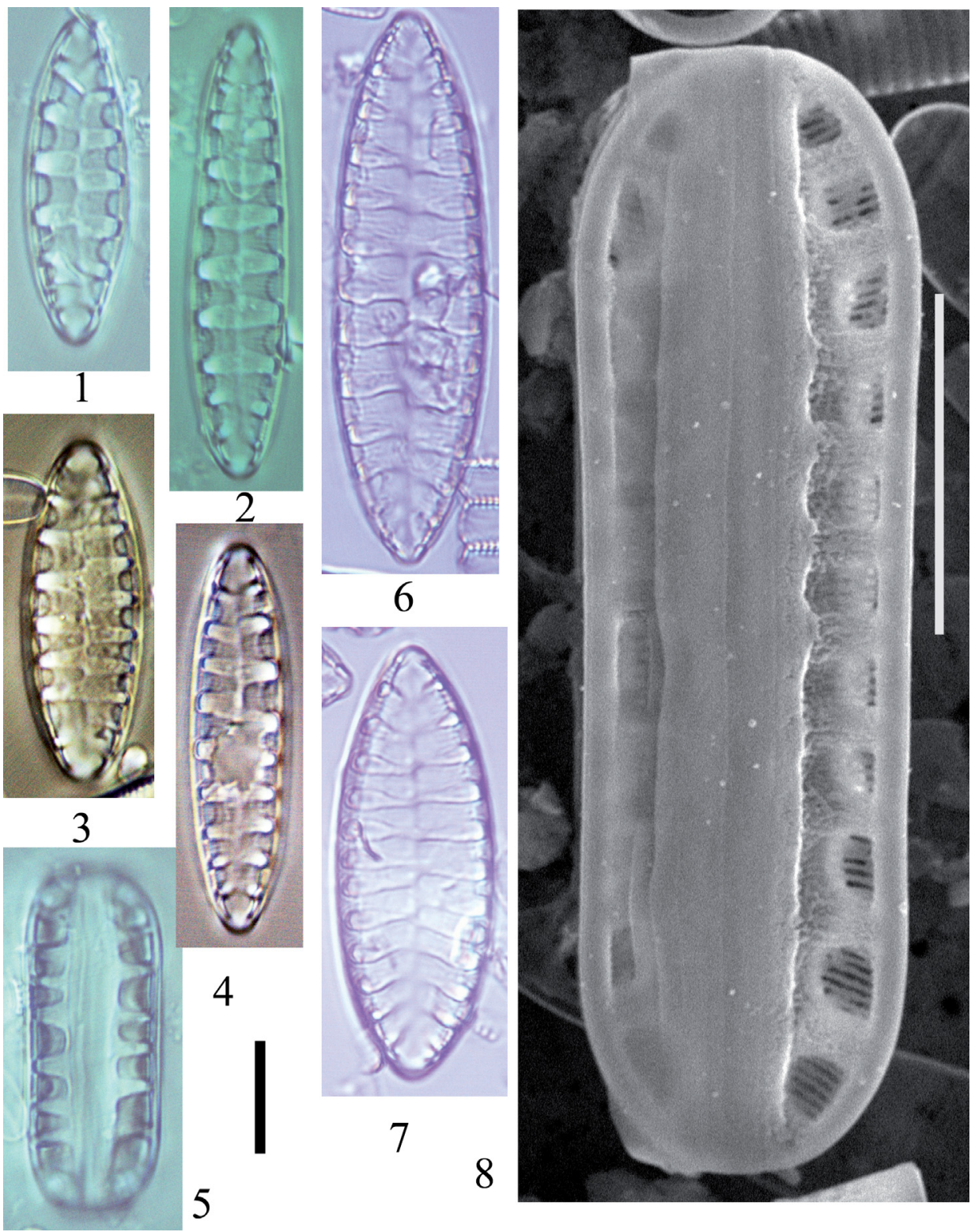

Plate 81: Surirella linearis. - Figs 1-5: Lake Lezilor, LM. Figs 6-7: Lake Pietrele, LM. Fig. 8: Lake Lezilor, outside view, girdle view, SEM. Scale bars $=10 \mu \mathrm{m}$. 
Surirella linearis W. Smith 1853: 31, pl. 8: fig. 58

(Plate 82: Figs 1-6)

References: Smith (1853), HofmANn et al. (2013).

Distribution in glacial lakes in the Retezat Mountains

Lakes

Ana, Bucura, Florica, Gales, Gemenele, Lezilor, Lia, Negru, Pele-

aga, Pietrele, Pietrelice-2, Slavieu, Stanisoara, Stevia, Stirbu

Relative abundance (max.) $\quad 4.5 \%$

Constancy

$4(65 \%)$

Remarks: PÉTERFi (1993) reported this species from mires, lakes and also from running waters. This is a very common species in our recent study. 

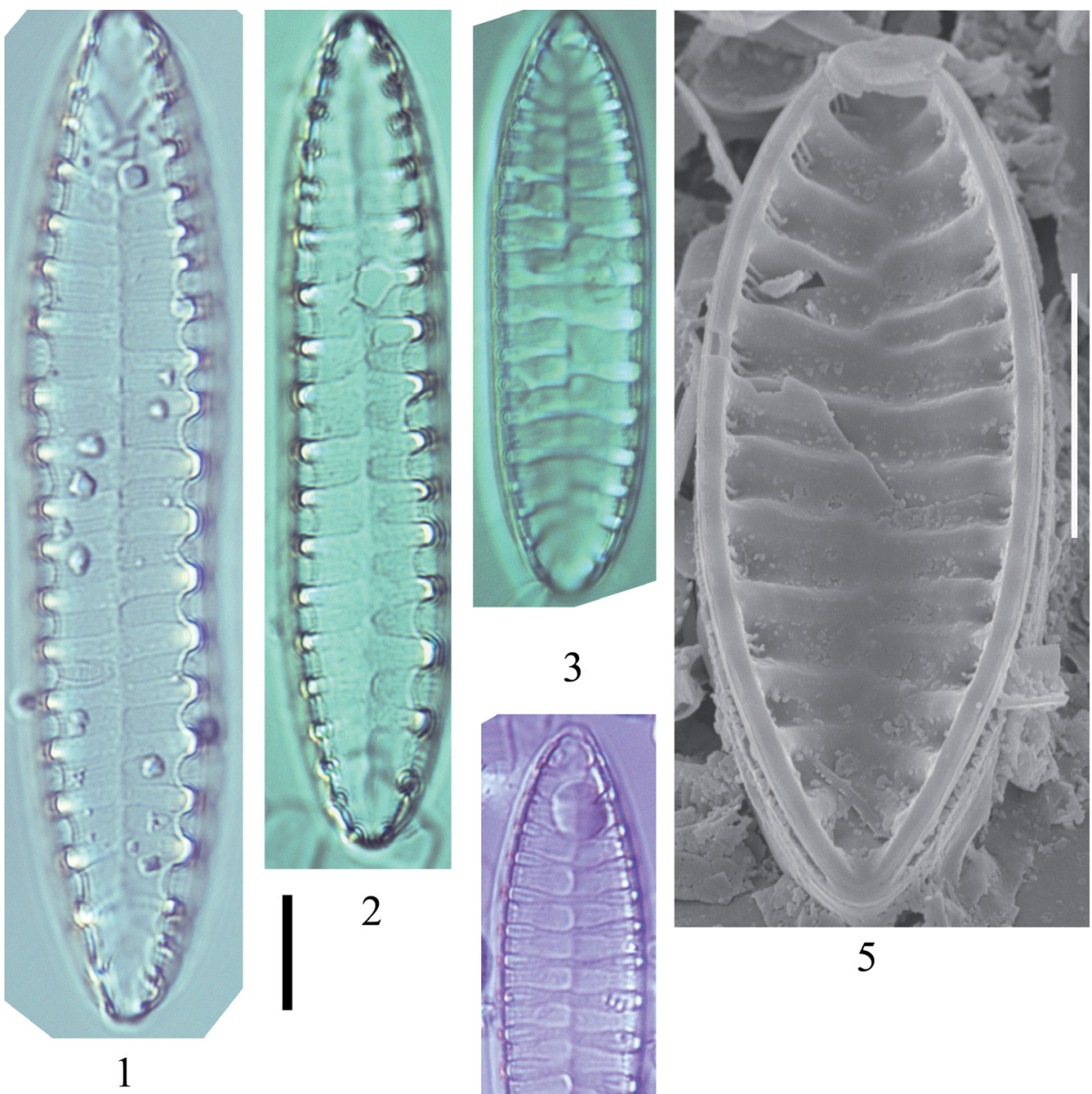

5

4

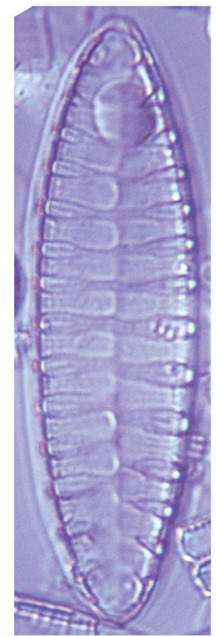

Plate 82: Surirella linearis. - Figs 1-3: Lake Lia, LM. Fig. 4: Lake Pietrele, inside view, SEM. Fig. 5: Lake Stevia, girdle view, SEM. Scale bars $=10 \mu \mathrm{m}$. 
Tabellaria flocculosa (Roth) Kützing 1844: 127, pl. 17: fig. 21 (Plate 83: Figs 1-10)

References: KÜtZing (1844), DeColibus (2013), Hofmann et al. (2013).

Distribution in glacial lakes in the Retezat Mountains

\begin{tabular}{ll}
\hline Lakes & Ana, Bucura, Caprelor, Gales, Gemenele, Lezilor, Lia, Peleaga, \\
& Peleguta, Slavieu, Turcelu, Viorica, Zanoaga \\
Relative abundance (max.) & $4.9 \%$ \\
Constancy & $3(57 \%)$ \\
\hline
\end{tabular}

Remarks: PÉTERFI (1993) reported this species from mires. Very common, sometimes quite abundant species in the Retezat Mts. 

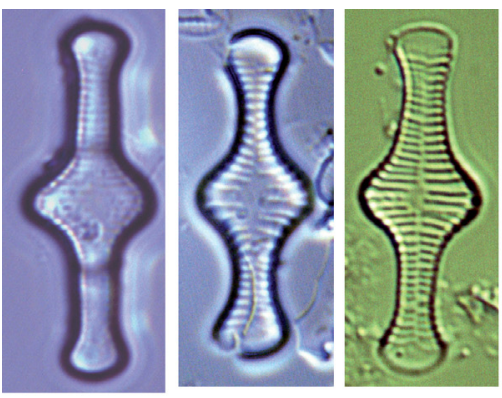

3

$1-2$
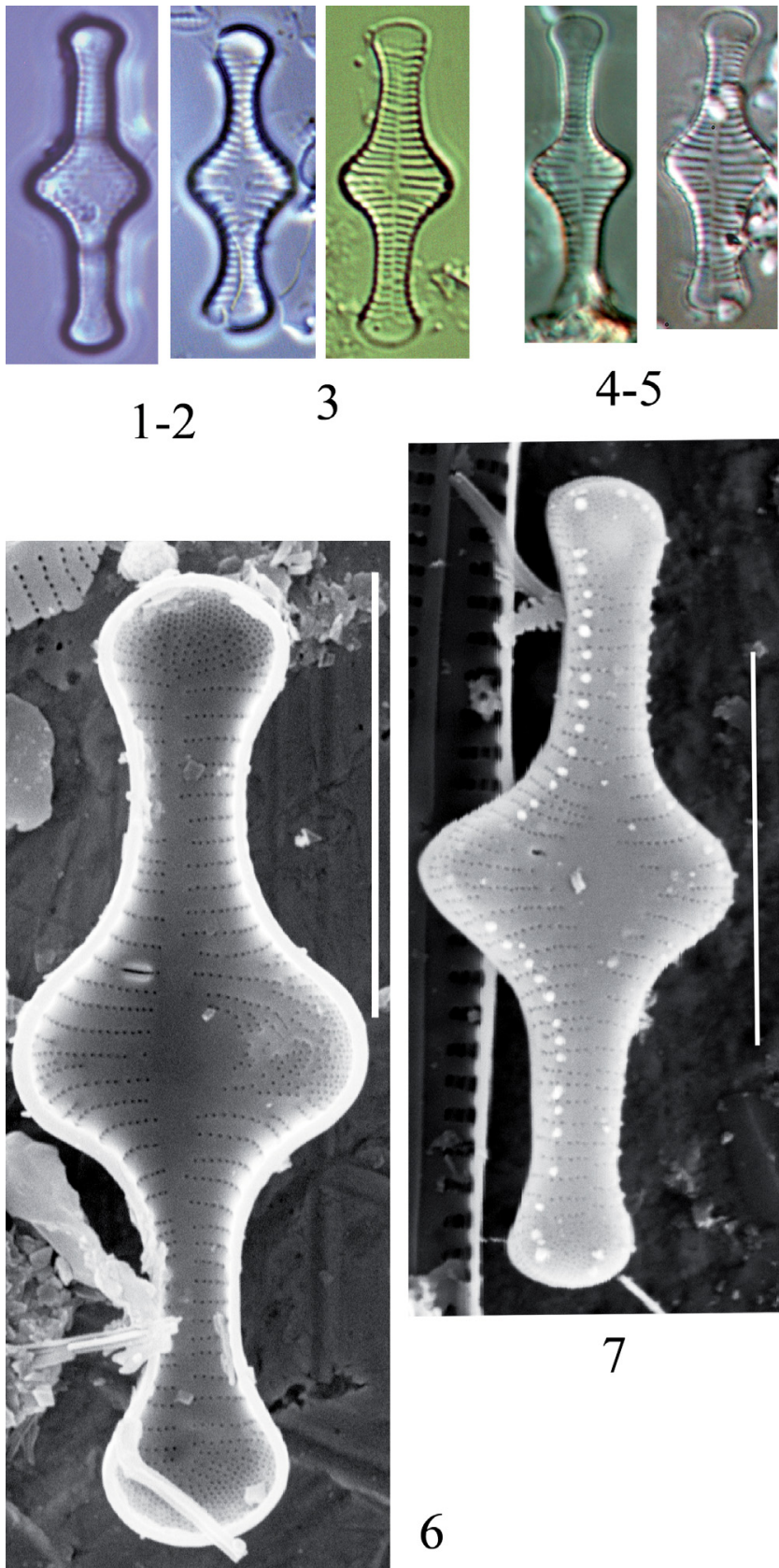

4-5

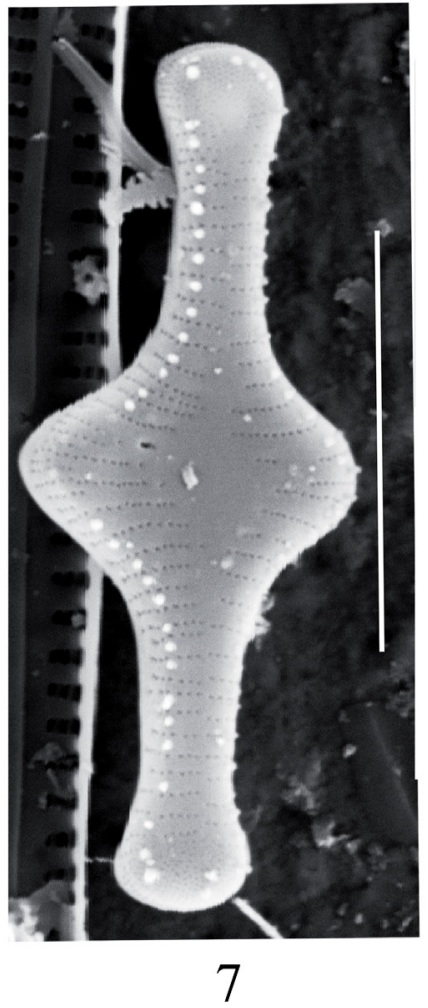

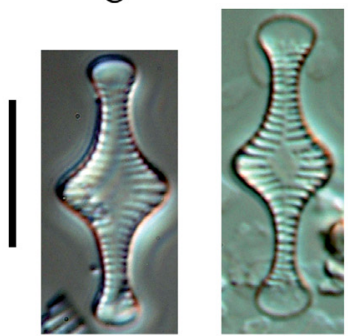

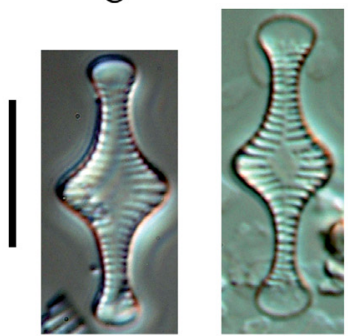

9-10

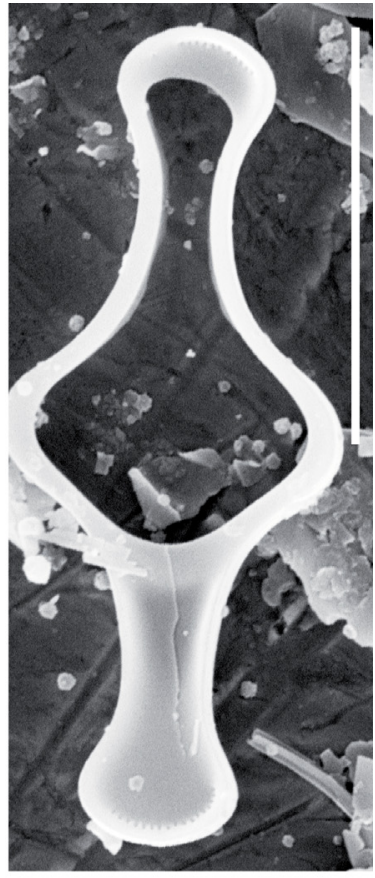

8

$$
0
$$

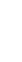

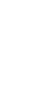

Plate 83: Tabellaria flocculosa. - Figs 1-3: Lake Zanoaga, LM. Figs 4-5: Lake Brazi, LM. Fig. 6: Lake Zanoaga, inside view, SEM. Fig. 7: Lake Gales, outside view, SEM. Fig. 8: Lake Brazi, a closed band with septa, SEM. Figs 9-10: Lake Gales, LM. Scale bars $=10 \mu \mathrm{m}$. 


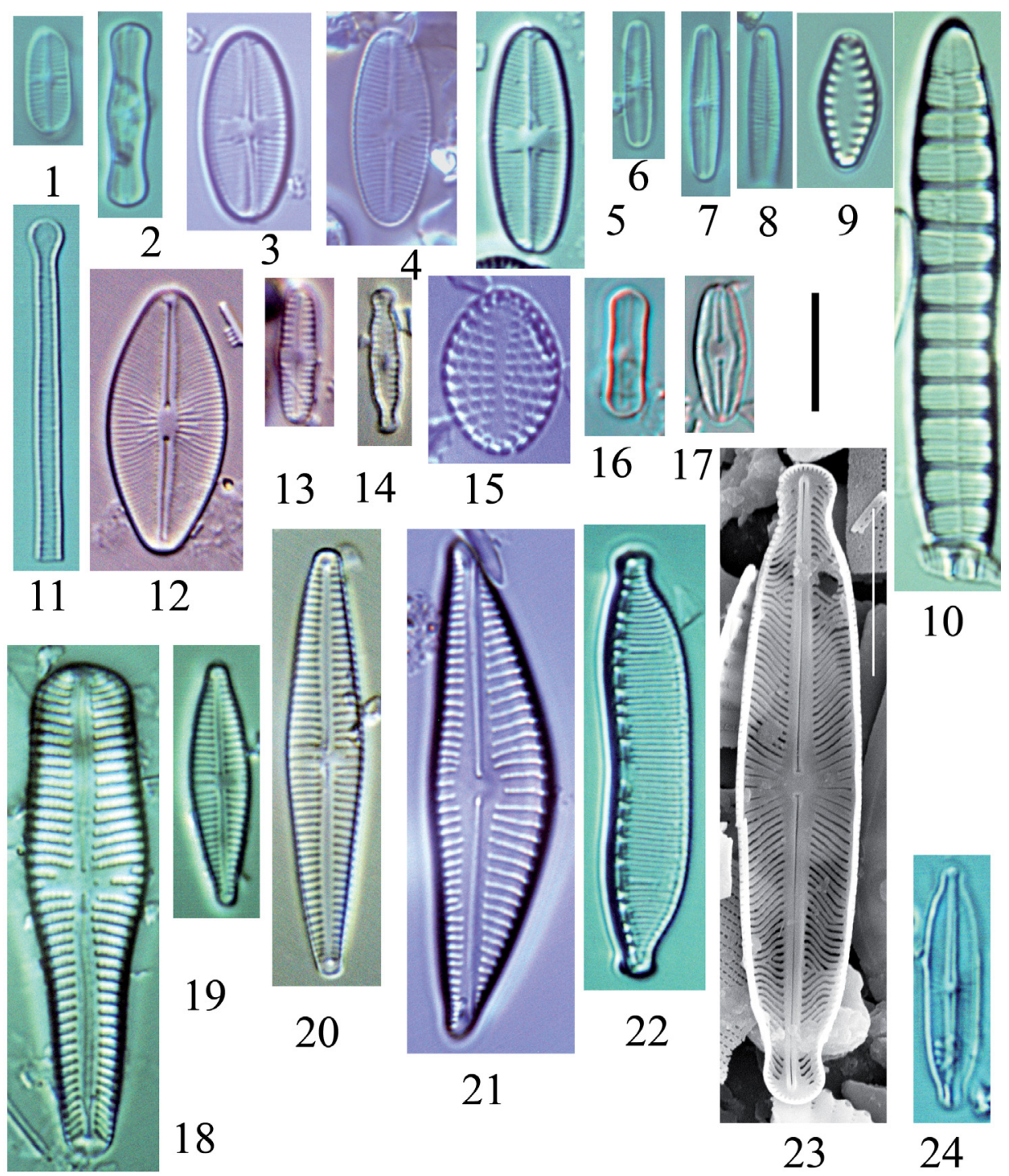

Plate 84: - Fig 1: Achnanthes didyma, Lake Gales. Fig. 2: Achnanthes trinodis, Lake Stanisoara. Figs 3-5: Achnanthidium bioretii, Lake Caprelor. Figs 6-8: Achnanthidium lineare, Lake Peleaga. Fig. 9: Pseudostaurosira brevistriata, Lake Caprelor. Fig. 10: Diatoma hyemale, Lake Lezilor. Fig. 11: Asterionella formosa, Lake Lezilor. Fig. 12: Cavinula cocconeiformis, Lake Lia. Fig. 13: Chamaepinnularia mediocris, Lake Bucura. Fig. 14: Chamaepinnularia soehrensis, Lake Bucura. Fig. 15: Cocconeis disculus, Lake Lia. Fig. 16: Diadesmis contenta var. biceps, Lake Gemenele. Fig. 17: Humidophila perpusilla, Lake Turcelu. Fig. 18: Gomphonema pala, Lake Ana. Fig. 19: Gomphonem cf. parvulum, Lake Zanoaga. Fig. 20. Gomphonema hebridense, Lake Peleaga. Fig. 21: Encyonema hebridicum, Lake Slavieu. Fig. 22: Hantzschia amphioxys, Lake Gales. Fig. 23: Kobayasiella parasubtilissima, Lake Stirbum, SEM. Fig. 24: Kobayasiella parasubtilissima, Lake Stirbu, LM. Scale bars $=10 \mu \mathrm{m}$. 

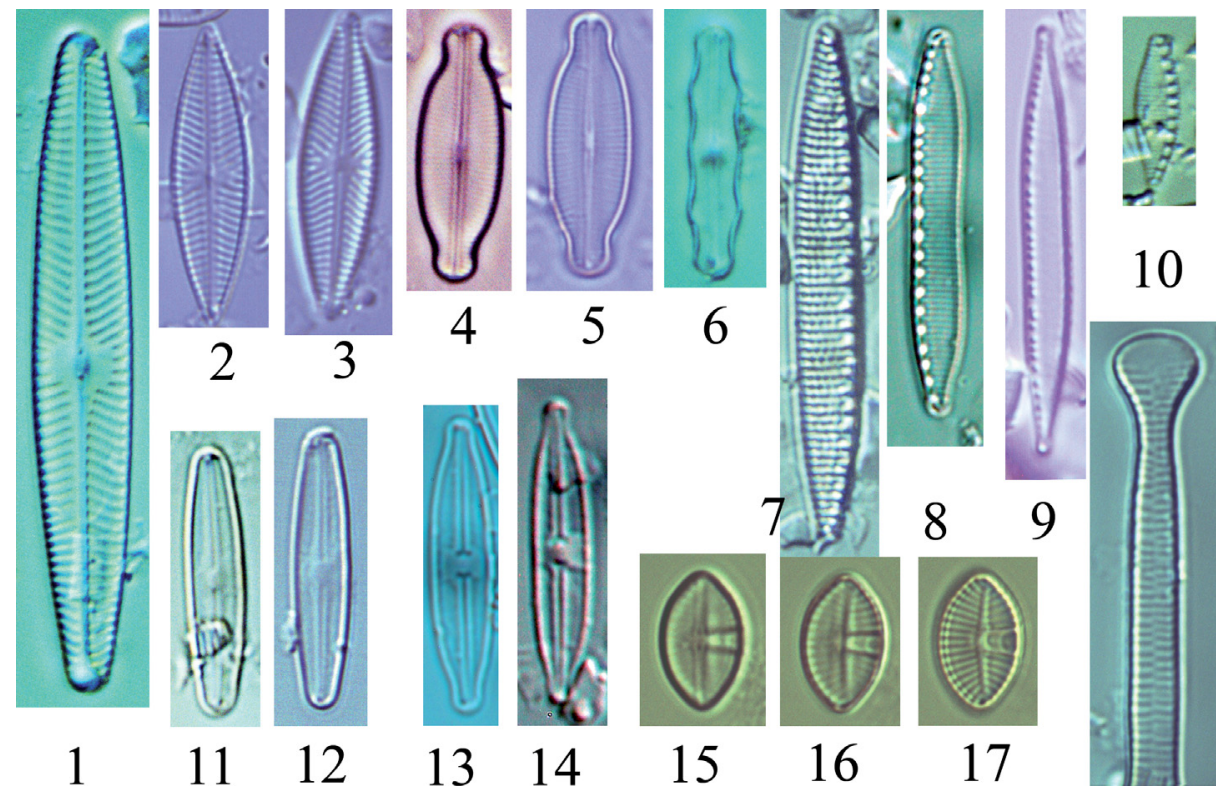

$\begin{array}{lll}1 & 11 & 12\end{array}$
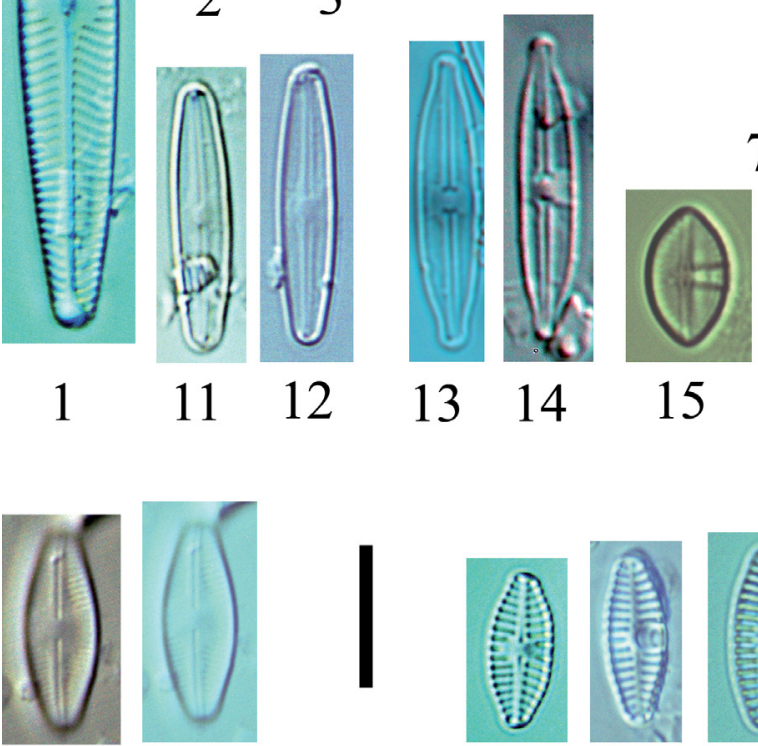

$13 \quad 14$

15
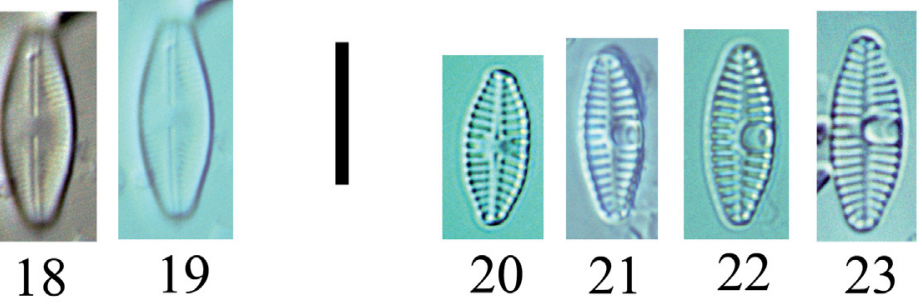

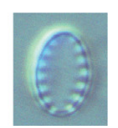

24

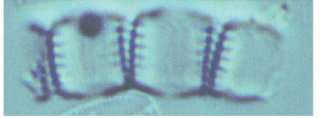

25

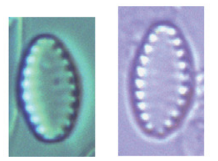

26

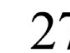

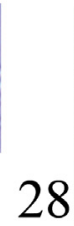
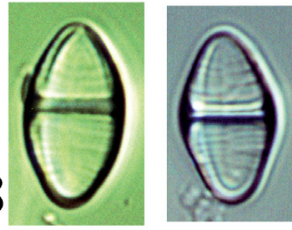

29

Plate 85: - Fig. 1: Navicula angusta, Lake Negru. Figs 2-3: Navicula cryptotenella, Lake Viorica. Figs 4-5: Navicula detenta, Lake Peleguta. Fig. 6: Sellaphora tridentula, Lake cf. Viorica. Fig. 7: Nitzschia amphibia, Lake Gales. Fig. 8: Nitzschia hantzschiana, Lake Gales. Fig. 9: Nitzschia palea var. debilis, Lake Florica. Fig. 10: Nitzschia frustulum, Lake Capleror. Figs 11-12: Nupela fennica, Lake Lia. Fig. 13: Nupela sp. 1, Lake Brazi. Fig. 14: Nupela sp. 2, Lake Gales. Figs 15-17: Planothidium oestrupii, Lake Lia. Figs 18-19: Psammothidium montanum, Lake Pietrelice. Figs 20-23: Planothidium freqentissimum, Lake Lia. Figs 24-27: Pseudostaurosiropsis cf. sp. Lake Peleguta. Figs 28-29: Tetracyclus rupestris, Lake Lia. Fig. 30: Tabellaria fenestrata Lake Slavieu. Scale bars $=10 \mu \mathrm{m}$. 


\section{DISCUSSION}

This compilation is the first illustrated guide to the diatom flora of the glacial lakes of the Retezat Mts, in the South Carpathians. In the last couple of years there has been a growing demand for the finer recognition of siliceous algae; therefore several richly illustrated publications have been prepared (e.g. POTAPOVA 2014, STENGER-KovÁcs and LENGYEL 2015). The special importance of such kind lies in the fact that images of these diatoms are now available for comparison and future biogeographical studies.

Summary of the diatom assemblages of glacial lakes in the Retezat Mts

The Retezat Mountains has a diverse diatom flora with a high level of species richness. The dominant genera in decreasing order by taxon numbers are Sellaphora, Encyonema, Aulacoseira, Gomphonema, Humidophila, Amphora, and while by relative abundance Psammothidium, Aulacosiera, Staurosira, Stauroforma.

Psammothidium Bukhtiyarova et Round (1996:3) species are very abundant (up to $60 \%$ relative abundance) and diverse in the studied lakes. Psammothidium was separated from Achnanthidium by BukntiYarova and Round (1996) to designate adnate taxa commonly attached to sand grains by the raphe valve face, as opposed to Achnanthidium species, which are more common on stable surfaces such as rocks and plants and found attached via short mucilaginous stalks. Psammothidium scotica, P. subatomoides and P. microscopica are the most common and abundant representatives of the genus, which was represented in the samples by a total of 13 species based on the AlgaeBase catalogue (GUIRY and GUIRY 2016). Some of the taxa, such as Achnanthes didyma and $A$. bioretti, will presumably be transferred to Psammothidium. However, the Achnanthidium taxa were less well represented in the samples, with $A$. minutissimum s. 1. and $A$. linearis.

The genus Aulacoseira was represented by eleven species, among those $A$. alpigena was especially abundant in the lakes. $A$. pfaffiana was also a common species in the lakes, together with $A$. perglabra and $A$. ambigua. A. valida usually appeared to be the most frequent in deeper lakes.

A number of Navicula and Gomphonema taxa are found in the samples are not identified to species level. They often did not form large enough population to be reliably identifiable.

Two of the 216 taxa are presumably new to science based on their unequivocally different features (one Humidophila, and one Staurosirella species). Their formal descriptions with morphometric analyses are in progress.

In conclusion, the number of 216 diatom taxa enumerated and documented here consists of 63 taxa mentioned only by PÉTERFi (1993), 74 taxa found and 
determined only by the author of this compilation, and 77 diatom taxa mentioned by both. The 216 diatom species and intraspecific taxa do not represent the entire diatom flora of the Retezat Mts. As highlighted before, the present compilation does mention neither the 34 Eunotia and 31 Pinnularia taxa recorded by PÉTERFI (1993), nor the representatives of these two genera identified by the author. To these numbers also add up some uncertain and/or unpublished data, resulting in a total number of diatom taxa exceeding 300 for the Retezat Mts. However, the compilation documents the common species and many of the rare ones inhabiting the investigated area, with special emphasis on small species, not easily identifiable by LM.

Obviously, the exploration of the diatom flora of the Retezat Mts is incomplete. Here we focused only on the siliceous algae of lakes that are usually less diverse habitats than mires and running waters. Péterfi reported altogether 64 diatom taxa from lakes, 52 from mires and 94 from running waters (PÉTERFI 1993). His results can be regarded as indicators of the unexplored status of the flora. Only five of the lakes are included in both surveys (PÉTERFI 1993 and the present one), namely the Lake Gales (2040), Lake Negru (2036), Lake Bucura (2041), Lake Zanoaga (1997) and Lake Zanogutii (1890), while Lake Valea Rea (2107), Lake Gemenele (1920), Lake Secat (2090), Lake Ghimelui (2116) and Lake Urit (2100) were studied only by PÉTERFI (1993). Altogether, we have diatom data from 28 of the 58 permanent lakes of the Retezat Mts.

A number of Navicula, Sellaphora and Gomphonema taxa found in the samples are not yet identified to species level. The revision of these genera is in progress.

Currently, the identification of siliceous algae is performed mainly on the basis of valve morphology. Although, the new generation methods (e.g. DNA barcoding) provide new information on the different taxa's identity and evidently are promising in exploring the diversity of microscopic plants (TAPOLCZAI et al. 2016), it still seems that for palaeoecological reconstructions there is no better approach than the morphology based identification.

The present work strongly connects to two on-going projects. The first is a larger scale palaeolimnological study on mountain lakes of the Carpathian Mountains, which extends the investigations both regarding the studied organisms (to cladocerans and chironomids, besides diatoms) and the sampling areas (to the Parâng and the Făgăras Mountains, besides the Retezat Mountains). The main aim of this extended multi-proxy project is to build a database (training set) for reliable quantitative climate reconstructions. The assessment and publication of the results of the whole project, together with a detailed set of lake water and sediment chemistry analyses results are in progress (Korponai, pers. comm.). 
These results also connect to the PROLONG project, or "Providing long environmental and genetic records of glacial and interglacial climatic oscillations and human impact in the Carpathian Basin". According to the main aim of PROLONG research project, palaeolimnological methods are used on lake sediments for palaeoecological reconstruction in the Retezat National Park. (https://sites.google.com/site/enikomagyaripollen/cv/prolong).

Last but not least, this publication is the summary and compilation of the five-years-long project called "Diatom based palaeoenvironmental reconstruction of mountain and lowland lakes in the Carpathian Basin" financed by the Hungarian National Scientific Fund, and conducted between 2011 and 2016.

Acknowledgements - The author is grateful to Csilla Kövér, János Korponai and Sándor Harangi for the field studies and sampling. Special thanks goes to Enikő Magyari for inviting me onto the PROLONG project. Lídia Szigyártó, Virág Pozderka and Viktória B-Béres helped in the editing of the manuscript and data checking. Valéria Pálházi and Erzsébet György carried out the slides preparation procedure. I would also like to express my thanks to the administration staff of the Retezat National Park. The final form of this compilation was significantly improved by the two anonymous reviewers, and László Lőkös and Zoltán Barina the editors of Studia botanica hungarica. Their contribution is very much acknowledged. This study was financed by the Hungarian Scientific Research Fund (OTKA 83999).

Összefoglaló: A magashegyi tavak mind a neo- mind a paleolimnológiai kutatásokban kiemelt fontosságúak. Ennek ellenére, a Déli-Kárpátok gleccsertavak kovavázas algáinak kutatása jóval elmarad más európai hegységekben lévő tavakéitól. Jelen munkában a Retyezát 58 állandó tava közül huszonháromnak a diatóma flórájáról számoltunk be. A fahatár fölött elhelyezkedő tavak (1740-2122 m tszf.) legmélyebb pontjairól, 2011 és 2014 között gyüjtött felszíni üledékmintákban vizsgáltuk a diatóma közösségeket. A 23-ból egyetlen tó, a Taul dintre Brazi - amint a neve is jelzi - a Fenyők-közti-tó található a fahatár alatt. A korábban közölt eredmények nómenklatúrai revíziója után összefoglaltuk a hegység kovaalga flóráját, a két legnépesebb nemzetség, az Eunotia és Pinnularia képviselői nélkül, melyek később kerülnek publikálásra. Ebben a munkában elsősorban a kis méretű fajok $(<20 \mu \mathrm{m})$ tisztázására és dokumentálására törekedtünk, amelyek határozásához a szkenning elektronmikroszkóp sokszor elengedhetetlen. 152 taxont határoztunk meg. Ebből a 79-ről adunk részletes LM és SEM dokumentációt. 40 további taxont fénymikroszkóp segítségével mutatunk be. Összesen 752 fény- és 187 szkenning elektronmikroszkópos kép illusztrálja az összefoglalót. Így, a korábbi adatokkal együtt 217 kovaalga vált ismertté a Retyezátból. Részletes fajleírás nélkül bemutatunk egy-egy Humidophila és Staurosira fajt, amelyek taxonómiai pozíciója még tisztázásra vár. Bevezetünk egy új kombinációt az Achnanthes helvetica var. minor-ra, mint Psammothidium helveticum var. minor.

\section{REFERENCES}

Aboal, M., Alvarez Cobelas, M., Cambra, J. and Ector, L. (2003): Floristic list of non-marine diatoms (Bacillariophyceae) of Iberian Peninsula, Balearic Islands and Canary Islands. Updated taxonomy and bibliography. - Diatom Monogr. 4: 1-639. 
AgARDh, C. A. (1831): Conspectus criticus diatomacearum. - Literis Berlingianus, Lundae, Vol. 3, pp. 33-48.

Álvarez-Blanco, I. and Blanco, S. (2013): Nitzschia imae sp. nov. (Bacillariophyta, Nitzschiaceae) from Iceland, with a redescription of Hannaea arcus var. linearis. - Anales Jardin Bot. Madrid 70: 144-151.

Andresen, N. A., Stoermer, E. F. and Kreis, R. J., JR. (2000): New nomenclatural combinations referring to diatom taxa which occur in The Laurentian Great Lakes of North America. Diatom Res. 15: 413-418. http://dx.doi.org/10.1080/0269249x.2000.9705505

BAhls, L. (2011a): Boreozonacola hustedtii. - Diatoms of the United States. http://westerndiatoms.colorado.edu/taxa/species/boreozonacola_hustedtii (accessed: 26.03.2016)

BAHLs, L. (2011b): Stauroneis phoenicenteron. - Diatoms of the United States. http://westerndiatoms.colorado.edu/taxa/species/stauroneis_phoenicenteron (accessed: 24.05 .2016 )

BAHLs, L. (2012a): Cymbopleura apiculata. - Diatoms of the United States. http://westerndiatoms. colorado.edu/taxa/species/cymbopleura_apiculata (accessed: 24.05.2016)

BAHLs, L. (2012b): Cymbopleura naviculiformis. - Diatoms of the United States. http://westerndiatoms.colorado.edu/taxa/species/cymbopleura_naviculiformis (accessed: 24.05.2016)

BAHLs, L. L. (2015): Kurtkrammeria, a new genus of freshwater diatoms (Bacillariophyta, Cymbellaceae) separated from Encyonopsis. - Nova Hedwigia 101: 165-190. http://dx.doi.org/10.1127/nova_hedwigia/2015/0263

Battarbee, R. W. (1986): Diatom analysis. - In: Berglund, B. E. (ed.): Handbook of Holocene palaeoecology and palaeohydrology. John Wiley and Sons, Chichester, New York, Brisbane, Toronto, Singapore, pp. 527-570.

Bishop, I. (2014): Fragilariforma bicapitata. - Diatoms of the United States. http://westerndiatoms.colorado.edu/taxa/species/fragilariforma_bicapitata (accessed 24.05.2016)

Blanco, S. (2016): A nomenclatural note on two species of the Achnanthidiaceae (Bacillariophyta). - Notulae algarum 4: 1-2.

Bory de Saint-Vincent, J. B. G. M. (1824): Diatome. Diatoma. - In: Audouin, I. et al. (eds): Dictionnaire classique d'histoire naturelle. CRA-D. 5, Rey et Gravier, Baudouin Frères, Paris, 461 pp.

BRÉBISSON, L. A. DE (1838): Considerations sur les diatomées et essai d'une classification des genres et des espèces appartenant à celle famille, par A. de Brébisson, auteur de la Flore de Normandie. - Falaise \& Paris: Brée l'Ainée Impr-Libr.; Meilhac. 4: 1-20. http://dx.doi.org/10.5962/bhl.title.64353

Buczkó, K., Magyari, E. K., Braun, M. and BÁlint, M. (2013a): Diatom-inferred lateglacial and Holocene climatic variability in the South Carpathian Mountains (Romania). - 2uaternary International 293: 123-135. http://dx.doi.org/10.1016/j.quaint.2012.04.042

Buczkó, K., Magyari, E. K., Hübener, T., Braun, M., Bálint, M., Tóth, M. and Lotter, A. F. (2012): Responses of diatoms to the Younger Dryas climatic reversal in a South Carpathian mountain lake (Romania). - J. Paleolimnol. 48: 417-431. http://dx.doi.org/10.1007/s10933-012-9618-1

Buczkó, K., Magyari, E., Soróczki-Pintér, É., Hubay, K., Braun, M. and Bálint, M. (2009): Diatom-based evidence for abrupt climate changes during the Late Glacial in the Southern Carpathian Mountains. - Central Eur. Geol. 52: 249-268.

http://dx.doi.org/10.1556/ceugeol.52.2009.3-4.3

Buczkó, K., Ognjanova-Rumenova, N. and Magyari, E. (2010): Taxonomy, morphology and distribution of some Aulacoseira taxa in glacial lakes in the South Carpathian Region. Polish Bot. J. 55: 149-163.

Buczkó, K., Wojtal, A. Z., Beszteri, B. and Magyari, E. K. (2015): Morphology and distribution of Navicula schmassmannii Hustedt and its transfer to genus Humidophila. - Studia bot. bung. 46: 25-41. http://dx.doi.org/10.17110/studbot.2015.46.1.25 
Buczkó, K., Wojtal, A. Z. and Magyari, E. K. (2013b): Late Quaternary Nupela taxa of Retezat Mts (S. Carpathians), with description of Nupela pocsii sp. nov. (Bacillariophyceae). - Polish Bot. J. 58: 427-436. http://dx.doi.org/10.2478/pbj-2013-0059

Buczkó, K., Wojtal, A. Z. and Magyari, E. (2013c): Lectotypification, emended description and distribution of Planothidium distinctum (Achnanthidiaceae, Bacillariophyceae). - Phytotaxa 117: 1-10. http://dx.doi.org/10.11646/phytotaxa.117.1.1

BukhtiYarova, L. N. (1995): Novye taksonomischeskie kombinatsii diatomovykh vodoroslei (Bacillariophyta). [New taxonomic combinations of diatoms (Bacillariophyta)]. - Algologia 5: 417-424.

Bukhtiyarova, L. and Round, F. E. (1996): Revision of the genus Achnanthes sensu lato section Marginulatae Bukh. sect. nov. of Achnanthidium Kütz. - Diatom Res. 11: 1-30. http://dx.doi.org/10.1080/0269249x.1996.9705361

Cantonati, M., Van de Vijver, B. and Lange-Bertalot, H. (2009): Microfissurata gen. nov. (Bacillariophyta), a new diatom genus from dystrophic and intermittently wet terrestrial habitats. - J. Phycol. 45: 732-741. http://dx.doi.org/10.1111/j.1529-8817.2009.00683.x

Caraus, I. (2012): Algae of Romania. A distributional checklist of actual algae. Version 2.3, third revision. - University of Bacau, Bacau, $694 \mathrm{pp}$.

Catalan, J., Pla-Rabés, S. Wolfe, A. P., Smol, J. P., Rühland, K. M., Anderson, N. J., KopáČek, J., Stuchlík, E., Schmidt R. Koinig, K. A. Camarero, L., Flower, R .J., Heiri, O., Kamenik, Ch., Korhola, A., Leavitt, P. R., Psenner, R. and Renberg, I. (2013): Global change revealed by palaeolimnological records from remote lakes: a review. $-J$. Paleolimnol. 49: 513-535. http://dx.doi.org/10.1007/s10933-013-9681-2

Cholnoky, B. J. and Schindler, H. (1953): Die Diatomeengesellschaften der Ramsauer Torfmoore. - Sitzungsber. Osterreich. Akad. Wiss., Math.-Naturw. Kl. 162: 597-624.

Cleve, A. (1895): On recent freshwater diatoms from Lule Lapmark in Sweden. - Kongl. Sv. Vetensk. Handl. 21: 1-49.

Cleve, P. T. (1891): The diatoms of Finland. - Acta Soc. Fauna Flora Fenn. 8: 1-68. http://dx.doi.org/10.5962/bhl.title.64355

Cleve, P. T. (1894): Synopsis of the naviculoid diatoms. Part I. - Kongl. Sv. Vetensk. Handl. 26: 1-194. http://dx.doi.org/10.5962/bhl.title.68663

Cleve, P. T. and Grunow, A. (1880): Beiträge zur Kenntniss der arctischen Diatomeen. - Kungl. Sv. Vetensk. Handl. 417: 1-121.

Cleve, P. T. and Jentzsch, A. (1882): Über einige diluviale und aluviale Diatomeenschichten Norddeutschlands. - Schrift. Phys.-̈̈kon. Ges. Königsberg 22: 129-170.

Cleve, P. T. and MöLler, J. D. (1879): Diatoms. - Esatas Edquists Boktrykeri, Upsala, 5: 217-276.

Cleve-Euler, A. (1922): Diatomeen-verzeichnisse in "Beschreibung zur Kartenblatter Väse". Sveriges Geologiska Undersökning.

Cox, E. J. (1988): Placoneis Mereschkowsky: the re-evaluation of a diatom genus originally characterized by its chloroplast type. - Diatom Res. 2: 145-157. http://dx.doi.org/10.1080/0269249x.1987.9704994

Czarnecki, D. B. (1994): The freshwater diatoms culture collection at Loras College, Dubuque, Iowa. In: KocioleK, J. P. (ed.): Proceedings of the 11th International Diatom Symposium. Mem. California Acad. Sci. 17: 155-174.

DeColibus, D. (2013): Tabellaria flocculosa. - Diatoms of the United States. http://westerndiatoms.colorado.edu/taxa/species/tabellaria_flocculosa (accessed: 24.05.2016)

Desmazières, J. B. H. J. (1830): Plantes Cryptogames du nord de la France. - The author, Lille, pp. 451-500.

De TonI, G. B. (1891): Sylloge algarum omnium hucusque cognitarum. Vol. II. Sylloge Bacillariearum. Sectio I. Rhaphideae. - Sumptibus auctoris, Patavii, 490 pp. 
Edlund, M. B., Soninkhishig, N., Williams, R. M. and Stoermer, E. F. (2001): Biodiversity of Mongolia: checklist of diatoms, including new distributional reports of 31 taxa. - Nova Hedwigia 72: 59-90.

EHRENBERG, C. G. (1832): Über die Entwickelung und Lebensdauer der Infusionsthiere; nebst ferneren Beiträgen zu einer Vergleichung ihrer organischen Systeme. - Abh. König. Akad. Wiss. Berlin, Physik. Klasse 1831: 1-154.

Ehrenberg, C. G. (1838): Die Infusionsthierchen als vollkommene Organismen: Ein Blick in das tiefere organische Leben der Natur. - Verlag von Leopold Voss, Leipzig, 547 pp. http://dx.doi.org/10.5962/bhl.title.97605

Ehrenberg, C. G. (1843): Verbreitung und Einfluss des mikroskopischen Lebens in Süd-und Nord-Amerika. - Abh. Königl. Akad. Wiss. Berlin 1841: 291-466.

English, J. and Potapova, M. (2009): Aulacoseira pardata sp. nov., A. nivalis comb. nov., A. nivaloides comb. et stat. nov., and their occurrences in western North America. - Proc. Acad. Nat. Sci. Philadelphia 158: 37-48. http://dx.doi.org/10.1635/053.158.0102

English, J. and Potapova, M. (2010): Aulacoseira valida. - Diatoms of the United States. http:// westerndiatoms.colorado.edu/taxa/species/aulacoseira_valida (accessed: 24.05.2016)

Fallu, M. A., Allaire, N. and Pienitz, R. (2000): Freshwater diatoms from northern Québec and Labrador (Canada). Species-environment relationships in lakes of boreal forest, foresttundra and tundra regions. - Bibl. Diatomol. 45: 1-200.

Flower, R. J. and Jones, V. J. (1989): Taxonomic descriptions and occurrences of new Achnanthes taxa in acid lakes in the UK. - Diatom Res. 4: 227-239. http://dx.doi.org/10.1080/0269249x.1989.9705072

Flower, R. J., Jones, V. J. and Round, F. E. (1996): The distribution and classification of the problematic Fragilaria (virescens v.) exigua Grunow/Fragilaria exiguiformis (Grunow) Lange-Bertalot: a new species or a new genus. - Diatom Res. 11: 41-57. http://dx.doi.org/10.1080/0269249x.1996.9705363

Fourtanier, E. and Kociolek, J. P. (2009a): Catalogue of diatom names: part I: introduction and bibliography. - Occ. Pap. Calif. Acad. Sci. 156(1).

Fourtanier, E. and Kociolek, J. P. (2009b): Catalogue of diatom names: part II: Abas through Bruniopsis. - Occ. Pap. Calif. Acad. Sci. 156(2).

Germain, H. (1981): Flore des diatomées. Diatomophycées eaux douces et saumâtres du Massif Armoricain et des contrées voisines d'Europe occidentale. - Société Nouvelle des Éditions Boubée, Paris, $444 \mathrm{pp}$.

GREGORY, W. (1854): Notice of the new forms and varieties of known forms occurring in the diatomaceous earth of Mull; with remarks on the classification of the Diatomaceae. - 2uart. J. Microsc. Sci. 2: 90-100, pl. IV.

Gregory, W. (1856): Notice of some new species of British fresh-water Diatomaceae. - Quart. J. Microsc. Sci., n. ser. 4: 1-14.

Grunow, A. (1860): Über neue oder ungenügend gekannte Algen. Erste Folge, Diatomeen, Familie Naviculaceen. - Verh. Kaiser.-König. Zool.-Bot. Gesellsch. Wien 10: 503-582.

Grunow, A. (1862): Die Österreichischen Diatomaceen nebst Anschluss einiger neuen Arten von andern Lokalitäten und einer kritischen Uebersicht der bisher bekannten Gattungen und Arten. - Verh. Kaiser.-König. Zool.-Bot. Gesellsch. Wien 12: 315-472. http://dx.doi.org/10.5962/bhl.title.64361

Grunow, A. (1884): Die Diatomeen von Franz Josefs-Land. - Denkschr. Kaiser. Akad. Wiss., Math.Nat. Cl., Wien 48: 53-112.

Guiry, M. D. and Guiry, G. M. (2016): AlgaeBase. - World-wide electronic publication, National University of Ireland, Galway. http://www.algaebase.org (accessed: 25.04.2016) 
Hamilton, P. (2010): Brachysira brebissonii. - Diatoms of the United States. http://westerndiatoms.colorado.edu/taxa/species/brachysira_brebissonii (accessed: 24.05.2016)

Hamilton, P. B., De HaAn, M., Kopalová, K., Zidarova, R. and van De Vijver, B. (2013): An evaluation of selected Neidium species from the Antarctic region. - Diatom Res. 29: 27-40. http://dx.doi.org/10.1080/0269249x.2013.822020

Hamilton, P. B., Poulin, M., Charles, D. F. and Angell, M. (1992): Americanarum Diatomarum Exsiccata: CANA, Voucher Slides from Eight Acidic Lakes in Northeastern North America. - Diatom Res. 7: 25-36. http://dx.doi.org/10.1080/0269249x.1992.9705195

Hartley, B., Ross, R. and Williams, D. M. (1986): A check-list of the freshwater, brackish and marine diatoms of the British Isles and adjoining coastal waters. - J. Marine Biol. Assoc. UK 66: 531-610. http://dx.doi.org/10.1017/s0025315400042235

HASSALL, A. H. (1850): A microscopic examination of the water supplied to the inhabitants of London and the suburban districts; illustrated by coloured plates, exhibiting the living animal and vegetable productions in Thames and other waters, as supplied by the several companies; with an examination, microscopic and general, of their sources of supply, as well as the Henly-on-Thames and Watford plans, etc. - Samuel Highley, London, $66 \mathrm{pp}$.

Haworth, E. Y. (1990): Diatom name validation. - Diatom Res. 5: 195-196. http://dx.doi.org/10.1080/0269249x.1990.9705104

Heiberg, P. A. C. (1863): Conspectus criticus diatomacearum danicarum. Kritisk oversigt over de danske Diatomeer. - Wilhelm Priors Forlag, Kjøbenhavn, 135 pp. http://dx.doi.org/10.5962/bhl.title.68738

Hofmann, G., Werum, M. and Lange-Bertalot, H. (2013): Diatomeen im Süsswasser-Benthos von Mitteleuropa. - Koeltz Scientific Books, Königstein, 908 pp.

Hoidal, N. (2013): Meridion circulare. - Diatoms of the United States. http://westerndiatoms. colorado.edu/taxa/species/meridion_circulare_var._constrictum (accessed: 24.05.2016)

Houk, V. (1993): Some morphotypes in the "Orthoseira roeseana" complex. - Diatom Res. 8: 385-402. http://dx.doi.org/10.1080/0269249x.1993.9705269

Houk, V. (2003): Atlas of freshwater centric diatoms with a brief key and descriptions. Part I. Melosiraceae, Orthoseiraceae, Paraliaceae and Aulacoseiraceae. - Czech Phycol. (Suppl.) 1: $1-27$.

Hustedt, F. (1927): Die Kieselalgen Deutschlands, Österreichs und der Schweiz unter Berücksichtigung der übrigen Länder Europas sowie der angrenzenden Meeresgebiete. - In: RABENHORST, L. (ed.): Kryptogamen Flora von Deutschland, Österreich und der Schweiz. Vol. VII, Teil 1, Lief. 1. Akademische Verlagsgesellschaft m.b.h., Leipzig, pp. 1-272.

Hustedt, F. (1933):Die Kieselalgen Deutschlands, Österreichs und der Schweiz unter Berücksichtigung der übrigen Länder Europas sowie der angrenzenden Meeresgebiete. - In: RABENHORST, L. (ed.): Kryptogamen Flora von Deutschland, Österreich und der Schweiz. Vol. VII, Teil 2, Lief. 3. Akademische Verlagsgesellschaft m.b.h., Leipzig, pp. 321-432.

Hustedt, F. (1943): Die Diatomeenflora einiger Hochgebirgsseen der Landschaft Davos in den schweizer Alpen. - Int. Rev. ges. Hydrobiol. Hydrograph. 43: 124-197, 225-280. http://dx.doi.org/10.1002/iroh.19430430402

Hustedt, F. (1962): Die Kieselalgen Deutschlands, Österreichs und der Schweiz unter Berücksichtigung der übrigen Länder Europas sowie der angrenzenden Meeresgebiete. - In: RABENHORST, L. (ed.): Kryptogamen Flora von Deutschland, Österreich und der Schweiz. Vol. VII. Akademische Verlagsgesellschaft m.b.h., Leipzig, pp. 161-348.

Jancsik, P. (2007): A Retyezát-hegység. (The Retezat Mountains). - Pallas-Akadémia Könyvkiadó, Csíkszereda, $140 \mathrm{pp}$. 
JohANSEN, J. R. and SRAY, J. C. (1998): Microcostatus gen. nov., a new aerophilic diatom genus based on Navicula krasskei Hustedt. - Diatom Res. 13: 93-101. http://dx.doi.org/10.1080/0269249x.1998.9705436

KaWec Ka, B. (2012): Diatom diversity in streams of the Tatra National Park (Poland) as indicator of environmental conditions. - W. Szafer Institute of Botany, Polish Academy of Sciences, Kraków, 213 pp.

KaWecka, B. and Galas, J. (2003): Diversity of epilithic diatoms in high mountain lakes under the stress of acidification (Tatra Mts, Poland). - Ann. Limnol. Int. J. Limnol. 39: 239-253. http://dx.doi.org/10.1051/limn/2003019

KocioleK, P. (2010): Hannaea arcus. - Diatoms of the United States. http://westerndiatoms.colorado.edu/taxa/species/hannaea_arcus (accessed: 24.05.2016)

Kociolek, P. (2011a): Meridion circulare. - Diatoms of the United States. http://westerndiatoms. colorado.edu/taxa/species/meridion_circulare (accessed: 24.05.2016)

KocioleK, P. (2011b): Navicula rhynchocephala. - Diatoms of the United States. http://westerndiatoms.colorado.edu/taxa/species/navicula_rhynchocephala (accessed: 24.05 .2016 )

KocioleK, P. (2011c): Nitzschia perminuta. - Diatoms of the United States. http://westerndiatoms.colorado.edu/taxa/species/nitzschia_perminuta (accessed: 24.05.2016)

Kociolek, P. and GraefF, C. (2011): Frustulia crassinervia. - Diatoms of the United States. http:// westerndiatoms.colorado.edu/taxa/species/frustulia_crassinervia (accessed: 24.05.2016)

Kociolek, J. P. and Stoermer, E. F. (1987): Ultrastructure of Cymbella sinuata and its allies (Bacillariophyceae), and their transfer to Reimeria, gen. nov. - Syst. Bot. 12: 451-459. http://dx.doi.org/10.2307/2418882

Kövér, C. (2016): Magashegyi tavak paleoökológiai vizsgálata a Déli Kárpátokban. (Paleoecological investigation of mountain lakes in the Southern Carpathians). - University of West Hungary Doctoral School, PhD dissert., 112 pp.

Kövér, C., Korponai, J., Harangi, S. and Buczkó, K. (2015): A new European record of Diadesmis fukushimae and its transference to Humidophila genus (Bacillariophyta). - Acta Bot. Croat. 74: 245-252. http://dx.doi.org/10.1515/botcro-2015-0020

Krammer, K. (1980): Morphologic and taxonomic investigations of some freshwater species of the diatom genus Amphora Ehr. - Bacillaria 3: 197-225.

Krammer, K. (1991): Morphology and taxonomy of some taxa in the genus Aulacoseira Thwaites (Bacillariophyceae). I. Aulacoseira distans and similar taxa. - Nova Hedwigia 52: 89-112.

Krammer, K. (1997): Die cymbelloiden Diatomeen. Eine Monographie der welweit bekannten Taxa. Teil 1. Allgemeines und Encyonema Part. - Bibl. Diatomol. 36: 1-382.

Krammer, K. (2002): Cymbella. - In: LANge-Bertalot, H. (ed.): Diatoms of Europe. Diatoms of the European Inland waters and comparable habitats 3. ARG Gantner Verlag KG, Ruggell, $584 \mathrm{pp}$.

Krammer, K. (2003): Cymbopleura, Delicata, Navicymbula, Gomphocymbellopsis, Afrocymbella. In: LANGE-Bertalot, H. (ed.): Diatoms of Europe. Diatoms of the European Inland waters and comparable habitats 4. ARG Gantner Verlag KG, Ruggell, 529 pp.

Krammer, K. and Lange-Bertalot, H. (1985): Naviculaceae Neue und wenig bekannte Taxa, neue Kombinationen und Synonyme sowie Bemerkungen zu einigen Gattungen. - Bibl. Diatomol. 9: 9-230.

Krammer, K. and Lange-Bertalot, H. (1991): Bacillariophyceae 4. Teil: Achnanthaceae, Kritische Ergänzungen zu Navicula (Lineolatae) und Gomphonema. - In: EtTL, H., Gerloff, J., Heynig, H. and Mollenhauer, D. (eds): Süsswasserflora von Mittel-Europa, Band 2/4. Spektrum Akademischer Verlag, Heidelberg, 599 pp. 
Krammer, K. and Lange-Bertalot, H. (1999a): Bacillariophyceae 1. Teil: Naviculaceae. - In: Ettl, H., Gerloff, J., Heynig, H. and Mollenhauer, D. (eds): Süsswasserflora von Mittel-Europa, Band 2/1. Spektrum Akademischer Verlag, Heidelberg, 876 pp.

Krammer, K. and Lange-Bertalot, H. (1999b): Bacillariophyceae 2. Teil: Bacillariaceae, Epithemiaceae, Surirellaceae. - In: Ettr, H., Gerloff, J., Heynig, H. and Mollenhauer, D. (eds): Süsswasserflora von Mittel-Europa, Band 2/2. Spektrum Akademischer Verlag, Heidelberg, $611 \mathrm{pp}$.

Krammer, K. and Lange-Bertalot, H. (2000): Bacillariophyceae 3. Teil: Centrales, Fragilariaceae, Eunotiaceae. - In: Ettr, H., Gerloff, J., Heynig, H. and Mollenhauer, D. (eds): Süsswasser flora von Mittel-Europa, Band 2/3. Spektrum Akademischer Verlag, Heidelberg, $599 \mathrm{pp}$.

Krammer, K. and Lange-Bertalot, H. (2004): Bacillariophyceae 4. Teil: Achnanthaceae, Kritische Erganzungen zu Navicula (Lineolatae), Gomphonema Gesamtliteraturverzeichnis. Teil 1-4. 2nd revised edition. - In: Ettr, H., Gerloff, J., Heynig, H. and Mollenhauer, D. (eds): Süsswasser von Mitteleuropa, Band 2(4). Spektrum Akademischer Verlag, Heidelberg, 468 pp.

KRASS KE, K. (1938): Beiträge zur Kenntnis der Diatomeen-Vegetation von Island und Spitzsbergen. - Arch. Hydrobiol. 33: 503-533.

Kulikovskiy, M. S., Lange-Bertalot, H., Witkowski, A., Dorofeyuk, N. I. and Genkal, S. I. (2010): Diatom assemblages from Sphagnum bogs of the world. I. Nur bog in northern Mongolia. - Bibl. Diatomol. 55: 1-326.

Kulikovskiy, M. S., Lange-Bertalot, H., Metzeltin, D. and Witkowski, A. (2012): Lake Baikal: hotspot of endemic diatoms I. - Icon. Diatomol. 23: 1-861.

Kützıng, F. T. (1844): Die Kieselschaligen Bacillarien oder Diatomeen. - W. Köhne, Nordhausen, 152 pp. http://dx.doi.org/10.5962/bhl.title.64360

KÜTZING, F. T. (1849): Species algarum. - F. A. Brockhaus, Lipsiae, 922 pp. http://dx.doi.org/10.5962/bhl.title.60464

Lagerstedt, N. G. W. (1873): Sötvatens-Diatomaceer fran Spetsbergen och Beeren Eiland. - Bih. Kongl. Sv. Vetenskaps-Akad. Handl. 1(14): 1-52.

LANGe-Bertalot, H. (1993): 85 Neue Taxa und über 100 weitere neu definierte Taxa ergänzend zur Süsswasserflora von Mitteleuropa, Vol. 2/1-4. - Bibl. Diatomol. 27: 1-164.

Lange-Bertalot, H. (1999a): Neue Kombinationen von Taxa aus Achnanthes Bory (sensu lato). - In: LANGe-Bertalot, H. (ed.): Iconographia Diatomologica. Annotated Diatom Micrographs 6. Phytogeography, diversity, taxonomy. Koeltz Scientific Books, Königstein, pp. 270-283.

LANGE-Bertalot, H. (1999b): Kobayasiella nom. nov. ein neuer Gattungsname für Kobayasia Lange-Bertalot 1996. - Icon. Diatomol. 6: 272-275.

LANGe-Bertalot, H. (2001): Diatoms of Europe: Navicula sensu stricto, 10 genera separated from Navicula sensu lato, Frustulia. Vol. 2. - ARG Gantner Verlag KG, Ruggell, 526 pp.

Lange-Bertalot, H., BaK, M., Wit kows Ki, A. and Tagliaventi, N. (2011): Eunotia and some related genera. - In: LANGE-BERTALOT, H. (ed.): Diatoms of the European inland waters and comparable habitats. 6. Koeltz Botanical Books, Königstein, 747 pp.

Lange-Bertalot, H. and Genkal, S. I. (1999): Diatomeen aus Siberien. I. Insel im Arktischen Ozean (Yugorsky-Shar Strait). [Diatoms from Siberia I. Islands in the Arctic Ocean (Yugorsky-Shar Strait)]. - In: LANGE-Bertalot, H. (ed.): Iconographia Diatomologica. Annotated Diatom Micrographs 6. Phytogeography, diversity, taxonomy. Koeltz Scientific Books, Königstein, 303 pp. 
Lange-Bertalot, H. and Krammer, K. (1989): Achnanthes, eine Monographie der Gattung mit Definition der Gattung Cocconeis und Nachtragen zu den Naviculaceae. - Bibl. Diatomol. 18: 1-393.

LANGe-Bertalot, H. and Metzeltin, D. (1996): Indicators of oligotrophy. 800 taxa representative of three ecologically distinct lake types, carbonate buffered-oligodystrophic-weakly buffered soft water. - In: LANGe-Bertalot, H. (ed.): Iconographia Diatomologica. Annotated Diatom Monographs. Vol. 2. Ecology, diversity, taxonomy. Koeltz Scientific Books, Königstein, 390 pp.

Lange-Bertalot, H. and Moser, G. (1994): Brachysira. Monographie der Gattung. - Bibl. Diatomol. 29: 1-212.

Lange-Bertalot, H. and Werum, M. (2001): Diadesmis fukushimae sp. nov. and some new or rarely observed taxa of the subgenus Paradiadesmis Lange-Bertalot and Le Cohu. - Diatom 17: 3-19.

Levkov, Z. (2009): Amphora sensu lato. - In: LANGe-Bertalot, H. (ed.): Diatoms of Europe: Diatoms of the European inland waters and comparable habitats. Vol. 5. ARG Gantner Verlag KG, Ruggell, pp. 5-916.

Lowe, R. (2015). Microcostatus krasskei. - Diatoms of the United States. http://westerndiatoms. colorado.edu/taxa/species/microcostatus_krasskei (accessed: 23.03.2016)

Lowe, R. L., Kociolek, P., Johansen, J. R., VAN De Vijver, B., LANGe-Bertalot, H. and KopaLOVÁ, K. (2014): Humidophila gen.nov., a new genus for a group of diatoms (Bacillariophyta) formerly within the genusDiadesmis: species from Hawai' $i$, including one new species. - Diatom Res. 29: 351-360. http://dx.doi.org/10.1080/0269249x.2014.889039

Lund, J. W. G. (1946): Observations on soil algae I. The ecology, size and taxonomy of British soil diatoms. Part 2. - New Phytol. 45: 56-110. http://dx.doi.org/10.1111/j.1469-8137.1946.tb05047.x

Magyari, E., Bálint, M., Buczkó, K., JaKab, G. and Braun, M. (2016): Introduction to the reconstruction of the late-glacial and Holocene terrestrial and aquatic ecosystems in the Retezat Mountains, Romania. - 2uaternary International. (in press)

MANN, D. G. (1989): The diatom genus Sellaphora: separation from Navicula. - Brit. Phycol. J. 24: 1-20. http://dx.doi.org/10.1080/00071618900650011

MANN, D. G. and Droop, J. M. (1996): Biodiversity, biogeography and conservation of diatoms. Hydrobiologia 336: 19-32. http://dx.doi.org/10.1007/bf00010816

MaYama, S., IDEI, M., Osada, K. and NAGUmo, T. (2002): Nomenclatural changes for 20 diatom taxa occurring in Japan Diatom. - Jap. J. Diatom. 18: 89-91.

Meresch Kowsky, C. (1902): On Sellaphora, a new genus of diatoms. - Ann. Mag. Nat. Hist. 7: 185-195. http://dx.doi.org/10.1080/00222930208678565

Monnier, O., Lange-Bertalot, H., Hoffmann, L. and Ector, L. (2007): The genera Achnanthidium Kützing and Psammothidium Bukhtiyarova \& Round in the family Achnanthidiaceae (Bacillariophyceae): a reappraisal of the differential criteria. - Cryptogamie, Algol. 28: $141-158$.

Morales, E. A. (2001): Morphological studies in selected fragilarioid diatoms (Bacillariophyceae) from Conneticut waters (U.S.A.). - Proc. Acad. Nat. Sci. Philadelphia 151: 105-120. http://dx.doi.org/10.1635/0097-3157(2001)151[0105:msisfd]2.0.co;2

Morales, E. (2010a): Staurosira construens. - Diatoms of the United States. http://westerndiatoms. colorado.edu/taxa/species/staurosira_construens_var._venter (accessed: 24.05.2016)

Morales, E. (2010b). Staurosirella pinnata. - Diatoms of the United States. http://westerndiatoms.colorado.edu/taxa/species/staurosirella_pinnata (accessed: 24.05.2016) 
Morales, E. A. and Edlund, M. B. (2003): Studies in selected fragilarioid diatoms (Bacillariophyceae) from Lake Hovsgol, Mongolia. - Phycol. Res. 51: 225-239. http://dx.doi.org/10.1111/j.1440-1835.2003.tb00190.x

Morales, E., and Spaulding, S. (2011): Fragilariforma virescens. - Diatoms of the United States. http://westerndiatoms.colorado.edu/taxa/species/fragilariforma_virescens (accessed: 24.05. 2016)

Morales, E., and Spaulding, S. (2013): Stauroforma exiguiformis. - Diatoms of the United States. http://westerndiatoms.colorado.edu/taxa/species/stauroforma_exiguiformis (accessed: 24.05.2016)

Moser, G., LAnge-Bertalot, H. and Metzeltin, D. (1998): Insel der Endemiten Geobotanisches Phänomen Neukaledonien (Island of endemics New Caledonia, a geobotanical phenomenon). - Bibl. Diatomol. 38: 1-464.

Nakov, T., Guillory, W. X., Julius, M. L., Theriot, E. C. and Alverson, A. J. (2015): Towards a phylogenetic classification of species belonging to the diatom genus Cyclotella (Bacillariophyceae): Transfer of species formerly placed in Puncticulata, Handmannia, Pliocaenicus and Cyclotella to the genus Lindavia. - Phytotaxa 217: 249-264. http://dx.doi.org/10.11646/phytotaxa.217.3.2

Oltean, M. and Zanoschi, V. (1963): Observațiuni diatomologice în Bistrița şi afluenții săi în zona lacului de acumulare de la Bicaz. - Acta Bot. Horti Bucurest. 1: 175-185.

O'Meara, E. (1875): Report on the Irish Diatomaceae. - Proc. Royal Irish Acad., Ser. 2, 2: 235-425.

Østrup, E. (1902): Freshwater diatoms. In: Flora of Koh Chang. Part VII. Contributions to the knowledge of the Gulf of Siam. Preliminary report on botany., Results of Danish expedition to Siam (1899-1900). - Bot. Tidsskr. 25: 28-41. http://dx.doi.org/10.5962/bhl.title.55188

Østrup, E. (1910): Danske Diatoméer med 5 tavler et Engelsk résumé. Udgivet paa Carlsbergfondets bekostning. - C. A. Reitzel Boghandel Bianco Lunos Bogtrykkeri, Kjøbenhavn, 323 pp. http://dx.doi.org/10.5962/bhl.title.1044

Otu, M. and Spaulding, S. (2011a): Cavinula pseudoscutiformis. - Diatoms of the United States. http://westerndiatoms.colorado.edu/taxa/species/cavinula_pseudoscutiformis (accessed: 24.05.2016)

Otu, M. and Spaulding, S. (2011b): Navicula schmassmanni. - Diatoms of the United States. http://westerndiatoms.colorado.edu/taxa/species/Navicula_schmassmannii (accessed: 24. 05.2016)

Patrick, R. M. and Freese, L. R. (1961): Diatoms (Bacillariophyceae) from Northern Alaska. Proc. Acad. Nat. Sci. Philadelphia 112: 129-293.

PAtrick, R. and Reimer, C. W. (1966): The diatoms of the United States exclusive of Alaska and Hawaii. Vol. 1: Fragilariaceae, Eunotiaceae, Achnanthaceae, Naviculaceae. - Academy of Natural Sciences, Philadelphia, 688 pp. http://dx.doi.org/10.2307/1351135

Peragallo, M. (1903): Le Catalogue Général des Diatomées, 2. - Clermont-Ferrand, Paris, pp. 472-973.

PÉTeRfi, L. ŞT. (1966): Noi contribuții la cunoaşterea algelor din Munţii Retezatului. - Stud. Cerc. Biol., Ser. Bot. 18: 133-135.

PÉTERfi, L. ŞT. (1967): Date noi asupra florei algologice a Parcului Național Retezat. - Contrib. Bot. 1967: 287-295.

PÉTERFi, L. ŞT. (1974a): Preliminary notes on the subfossil and recent diatom flora of the Zanoguta peat bog from the Retezat mountains. - Stud. Univ. Babes-Bolyai, Ser. Biol. 19: 5-17.

PÉTERfi, L. ŞT. (1974b): Flora algală din complexul mlăştinos Valea Judele - Zănoaga, Parcul Național Retezat. - Acta Mus. Devensis, Sargetia X, Ser. Sci. Nat. 1974: 85-94. 
PÉTERFI, L. ŞT. (1993): Flora şi vegetația algală a mlaştinilor, lacurilor glaciare şi a apelor curgătoare. - In: Parcul Naţional Retezat. Studii Ecologice. Ed. West Side, Braşov, pp. 78-93.

PÉTERFI, L. ŞT. and NAGY-TóTH, F. (1963): Despre flora şi vegetaţia algologică a munților Retezat. - I. Acta Bot. Horti Bucurest. I: 107-130. (1961-1962).

Pfitzer, E. (1871): Untersuchungen über Bau und Entwickelung der Bacillariaceen (Diatomaceen). - Botanische Abhandlungen aus dem Gebiet der Morphologie und Physiologie. 2. Hrsg. J. Hanstein, Bonn, 189 pp.

Potapova, M. (without year): Karayevia oblongella. - Diatoms of the United States. http://westerndiatoms.colorado.edu/taxa/species/karayevia_oblongella (accessed: 28.04.2016)

Potapova, M. (2009a): Achnanthidium minutissimum. - Diatoms of the United States. http:// westerndiatoms.colorado.edu/taxa/species/Achnanthidium_minutissimum (accessed: 24. 05.2016)

Potapova, M. (2009b): Aulacoseira alpigena. - Diatoms of the United States. http://westerndiatoms.colorado.edu/taxa/species/Aulacoseira_alpigena (accessed: 24.05.2016)

Potapova, M. (2009c): Diatoma mesodon. - Diatoms of the United States. http://westerndiatoms. colorado.edu/taxa/species/Diatoma_mesodon (accessed: 24.05.2016)

Potapova, M. (2009d): Geissleria decussis. - Diatoms of the United States. http://westerndiatoms. colorado.edu/taxa/species/Geissleria_decussis.

Potapova, M. (2009e): Psammothidium subatomoides. - Diatoms of the United States. http:// westerndiatoms.colorado.edu/taxa/species/Psammothidium_subatomoides (accessed: 15. 05.2016)

Potapova, M. (2010a): Nupela lapidosa. - Diatoms of the United States. http://westerndiatoms. colorado.edu/taxa/species/nupela_lapidosa (accessed: 24.05.2016)

Potapova, M. (2010b): Nupela vitiosa. - Diatoms of the United States. http://westerndiatoms. colorado.edu/taxa/species/nupela_vitiosa (accessed: 24.05 .2016 )

Potapova, M. (2010c): Planothidium lanceolatum. - Diatoms of the United States. http://westerndiatoms.colorado.edu/taxa/species/planothidium_lanceolatum (accessed: 24.05.2016)

Potapova, M. (2010d): Psammothidium helveticum. - Diatoms of the United States. http://westerndiatoms.colorado.edu/taxa/species/psammothidium_helveticum (accessed: 24.05.2016)

Potapova, M. (2010e): Psammothidium levanderi. - Diatoms of the United States. http://westerndiatoms.colorado.edu/taxa/species/psammothidium_levanderi (accessed: 24.05.2016)

Potapova, M. (2010f): Psammothidium marginulatum. - Diatoms of the United States. http:// westerndiatoms.colorado.edu/taxa/species/psammothidium_marginulatum (accessed: 24 . 05.2016)

Potapova, M. (2010g): Psammothidium curtissimum. - Diatoms of the United States. http:// westerndiatoms.colorado.edu/taxa/species/psammothidium_curtissimum (accessed: 12.05. 2016)

Potapova, M. (2010h): Psammothidium scoticum. - Diatoms of the United States. http://westerndiatoms.colorado.edu/taxa/species/psammothidium_scoticum1 (accessed: 24.05.2016)

Potapova, M. (2011a): Navicula cryptocephala. - Diatoms of the United States. http://westerndiatoms.colorado.edu/taxa/species/navicula_cryptocephala (accessed: 24.05.2016)

Potapova, M. (2011b): Nupela impexiformis. - Diatoms of the United States. http://westerndiatoms.colorado.edu/taxa/species/nupela_impexiformis (accessed: 24.05.2016)

Potapova, M. (2014): Diatoms of Bering Island, Kamchatka, Russia. - Nova Hedwigia, Beih. 143: 63-102.

Potapova, M. and English, J. (2010): Aulacoseira ambigua. - Diatoms of the United States. http:// westerndiatoms.colorado.edu/taxa/species/aulacoseira_ambigua (accessed: 24.05.2016) 
Potapova, M. and English, J. (2011): Aulacoseira nivalis. -Diatoms of the United States. http:// westerndiatoms.colorado.edu/taxa/species/aulacoseira_nivalis (accessed: 24.05.2016)

Rabenhorst, L. (1848-1860): Die Algen Sachsens. - Resp. Mittel-Europa's Gesammelt und herausgegeben von Dr. L. Rabenhorst 1-100. No. 1-1000. Dresden.

Rabenhorst, L. (1853): Die Süsswasser Diatomaceen (Bacillarien) für Freunde der Mikroskopie. Eduard Kummer, Leipzig, 72 pp. http://dx.doi.org/10.5962/bhl.title.8348

RABENHORST, L. (1860): Erklärung der Tafel VI. - Hedwigia 2: 40.

Rabenhorst, L. (1863): Kryptogamen-Flora von Sachsen, Ober-Lausitz, Thüringen und NordBöhmen, mit Berücksichtigung der benachbarten Länder: erste Abtheilung: Algen im weitesten Sinne, Leber und Laubmoose. - Eduard Kummer, Leipzig, 653 pp. http://dx.doi.org/10.1002/ardp.18631640147

ReICHARDT, E. (2001): Revision der Arten um Gomphonema truncatum und G. capitatum. - In: Jahn, R., Kociolek, J. P., Wit kowski, A. and Compère, P. (eds): Lange-Bertalot Festschrift. Studies on diatoms dedicated to Prof. Dr. Dr. h.c. Horst Lange-Bertalot on the occasion of his 65th birthday. ARG Gantner Verlag KG, Ruggell, pp. 187-224.

REICHARDT, E. (2015): The identity of Gomphonema clavatum Ehrenberg (Bacillariophyceae) and typification of five species of the genus Gomphonema described by CG Ehrenberg. Diatom Res. 30: 141-149. http://dx.doi.org/10.1080/0269249x.2015.1009386

Reichardt, E. and Lange-Bertalot, H. (1991): Taxonomische Revision des Artencomplexes um Gomphonema angustum, G. dichotomum, G. intricatum, G. vibrio und ähnliche Taxa (Bacillariophyceae). - Nova Hedwigia 53: 519-544.

Reimer, C. W. (1959). The diatom genus Neidium. I. New species, new records and taxonomic revisions. - Proc. Acad. Nat. Sci. Philadelphia 111: 1-35.

Round, F. E., Crawford, R. M. and Mann, D. G. (1990): The diatoms: biology and morphology of the genera. - Cambridge University Press, Cambridge, 747 pp.

Schmidt, A. W. F. (1875a): Atlas der Diatomaceen-kunde. Ser. I, Heft 3. - Commissions-Verlag Von Ludwig Siever's Buchandlung, Aschersleben, pp. 9-12.

http://dx.doi.org/10.5962/bhl.title.64396

Schmidt, A. W. F. (1875b): Atlas der Diatomaceen-kunde. Ser. I, Heft 7. - Commissions-Verlag Von Ludwig Siever's Buchandlung, Aschersleben, pp. 25-28.

http://dx.doi.org/10.5962/bhl.title.64396

SChmidt, R., LANGe-Bertalot, H. and KLeE, R. (2004): Staurosira parasitoides sp. nova and Staurosira microstriata (Marciniak) Lange-Bertalot from surface sediment samples of Austrian alpine lakes. - Algol. Studies/Arch. Hydrobiol., Suppl. 114: 1-9.

http://dx.doi.org/10.1127/1864-1318/2004/0114-0001

Schoeman, F. R. and ARChibald, R. E. M. (1986): Observations on Amphora species (Bacillariophyceae) in the British Museum (Natural History). V. Some species from the subgenus Amphora. - S African J. Bot. 52: 425-437.

SIMONSEN, R. (1979): The diatom system: ideas on phylogeny. - Bacillaria 2: 9-71.

Siver, P. and Hamilton, P. B. (2005): Observation on new and rare species of freshwater diatoms from Cape Cod, Massachussets, USA. - Can. J. Bot. 83: 362-378. http://dx.doi.org/10.1139/b05-010

Siver, P. A., Hamilton, P. B., Stachura-Suchoples, K. and Kociolek, J. P. (2005): Diatoms of North America: The freshwater flora of Cape Cod, Massachusetts, USA. - In: LANGE-BERTALOT, H. (ed.): Iconographia diatomologica 14. Koeltz Scientific Books, Köningstein, Liechtenstein, $463 \mathrm{pp}$.

Smith, T. (2013): Pseudostaurosira pseudoconstruens. - Diatoms of the United States. http://westerndiatoms.colorado.edu/taxa/species/pseudostaurosira_pseudoconstruens (accessed: 24.05.2016) 
Sмith, W. (1853): A synopsis of the British Diatomaceae; with remarks on their structure, function and distribution; and instructions for collecting and preserving specimens. - John van Voorst, London, 89 pp. http://dx.doi.org/10.5962/bhl.title.10706

Sмith, W. (1855): Notes of an excursion to the south of France and the Auvergne in search of Diatomaceae. - Ann. Mag. Nat. Hist. 2: 1-9. http://dx.doi.org/10.1080/037454809495381

Soróczki-Pintér, É., Buczkó, K., Braun, M. and Magyari, E. K. (2012): Késő-glaciális és Holocén vízszintváltozások a Retyezátban egy gleccsertó kovaalga összetétele alapján. (Late glacial and Holocene diatom based lake level reconstruction in a glacial lake in Retezat Mountains (Romania). - Hidrol. Közl. 92: 64-67.

Soróczki-Pintér, É., MAgYari, E. K. and Buczkó, K. (2013): Preuve fondée sur les algues siliceuses de l'augmentation du niveau d'eau et du refroidissement à court terme autour de 9.2-ka dans les Carpates $d u$ Sud, Roumanie. (Siliceous algae based evidence for short-term lake level increase and cooling around 9.2-ka BP in the South Carpathian Mountains, Romania). - In: Rimet, F., Bouchez, A., Ector, L. and Montuelle, B. (eds): INRA Science and Impact, 7th Central European Diatom Meeting (CE-Diatom), 32nd meeting of the French-Speaking Diatomists Association (ADLaF), Thonon les Bains, France, 16-20 Sept. 2013, pp. 77-80.

Soróczki-Pintér, É., Pla-Rabes, S., Magyari, E. K., Stenger-Kovács, Cs. and Bucz kó, K. (2014): Late Quaternary Chrysophycean stomatocysts in a Southern Carpathian mountain lake, including the description of new forms (Romania). - Phytotaxa 170: 169-186. http://dx.doi.org/10.11646/phytotaxa.170.3.3

Stenger-Kovács, C. and Lengyel, E. (2015): Taxonomical and distribution guide of diatoms in soda pans of Central Europe. - Studia bot. hung. 46(Suppl.): 3-203.

http://dx.doi.org/10.17110/StudBot.2015.46.Suppl.3

StepaneK, J. and Kociolek, P. (2011): Amphora copulata. - Diatoms of the United States. http:// westerndiatoms.colorado.edu/taxa/species/amphora_copulata (accessed: 24.05.2016)

Tapolczai, K., Bouchez, A., Stenger-Kovács, C., Padisák, J. and Rimet, F. (2016): Traitbased ecological classifications for benthic algae: review and perspectives. - Hydrobiologia 776: 1-17. http://dx.doi.org/10.1007/s10750-016-2736-4

Van de Vijver, B., Ector, L., Beltrami, M. E., de HaAn, M., Falasco, E., Hlúbi ková, D., Jarlman, A., Kelly, M., Novais, M. H. and Wojtal, A. Z. (2011): A critical analysis of the type material of Achnanthidium lineare W. Sm. (Bacillariophyceae). - Algol. Studies 136: 167-191. http://dx.doi.org/10.1127/1864-1318/2011/0136-0167

Van Heurck, H. (1880): Synopsis des Diatomées de Belgique. Atlas. - Ducaju et Cie., Anvers, I-XXX pls.

Van Heurck, H. (1881): Synopsis des Diatomées de Belgique. Atlas. - Ducaju et Cie., Anvers, XXXI-LXXVII pls.

Van Heurck, H. (1885): Synopsis des Diatomées de Belgique. Texte. - Martin Brouwers \& Co., Anvers, 235 pp. http://dx.doi.org/10.5962/bhl.title.1990

Van Heurck, H. (1896): A treatise on the Diatomaceae. - William Wesley \& Son, London, 558 pp.

VeSELÁ, J. and Joh ANSEN, J. R. (2009): The diatom flora of ephemeral headwater streams in the Elbsandsteingebirge region of the Czech Republic. - Diatom Res. 24: 443-477.

http://dx.doi.org/10.1080/0269249x.2009.9705813

Werum, M. and LANGe-BerTALOT, H. (2004): Diatoms in springs from Central Europe and elsewhere under the influence of hydrologeology and anthropogenic impacts. - In: LANGE-BERTALOT H. (ed.): Iconographia Diatomologica. Annotated Diatom Micrographs 13. ARG Gantner Verlag KG, Ruggell, 417 pp.

Wetzel, C. E., Ector, L., VAn De Vijver, B., Compère, P. and Mann, D. G. (2015): Morphology, typification and critical analysis of some ecologically important small naviculoid species (Bacillariophyta). - Fottea 15: 203-234. http://dx.doi.org/10.5507/fot.2015.020 
Williams, D. M. and Round, F. E. (1988a): Revision of the genus Fragilaria. - Diatom Res. 2: 267-288. http://dx.doi.org/10.1080/0269249x.1987.9705004

Williams, D. M. and Round, F. E. (1988b): Fragilariaforma, nom. nov., a new generic name for Neofragilaria Williams and Round. - Diatom Res. 3: 265-267.

http://dx.doi.org/10.1080/0269249x.1988.9705039

WojtAL, A. Z. (2013): Species composition and distribution of diatom assemblages in spring waters from various geological formations in Southern Poland. - Bibl. Diatomol. 59: 1-436.

Wojtal, A. Z., Ognjanova-Rumenova, N., Wetzel, C. E., Hinz, F., Piatek, J., Kapetanovic, T., EстоR, L. and BuczKó, K. (2014): Diversity of the genus Genkalia (Bacillariophyta) in boreal and mountain lakes: taxonomy, distribution and ecology. - Fottea 14: 225-239. http://dx.doi.org/10.5507/fot.2014.017

(submitted: 25.04.2016, accepted: 31.07 .2016 )

\section{Index to taxa}

Achnanthes didyma Hustedt ................................................................................................................ 14

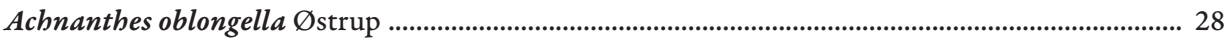

Achnanthes trinodis (Ralfs) Grunow ............................................................................................. 14

Achnanthes ventralis (Krasske) Lange-Bertalot ............................................................................ 14

Achnanthidium bioretii (H. Germain) Monnier, Lange-Bertalot et Ector ........................................ 14

Achnanthidium kryophila (J. B. Petersen) Bukhtiyarova ...................................................................... 15

Achnantbidium lineare W. Smith .................................................................................................. 15

Achnanthidium minutissimum (Kützing) Czarnecki ........................................................................... 30

Adlafia bryophila (Petersen) Gerd Moser, Lange-Bertalot et Metzeltin ........................................... 15

Adlafia minuscula (Grunow) Lange-Bertalot ................................................................................... 15

Amphipleura pellucida (Kützing) Kützing ………………............................................................... 15

Amphora copulata (Kützing) Schoeman et E. M. Archibald ............................................................. 32

Amphora inariensis Krammer ........................................................................................................ 34

Amphora indistincta Levkov ........................................................................................................... 15

Amphora pediculus (Kützing) Grunow ex A. Schmidt ................................................................... 15

Asterionella formosa Hassall ............................................................................................................ 15

Aulacoseira alpigena (Grunow) Krammer ........................................................................................... 36

Aulacoseira ambigua (Grunow) Simonsen …………………....................................................... 38

Aulacoseira granulata (Ehrenberg) Simonsen ..................................................................................... 15

Aulacoseira islandica (Otto Müller) ...................................................................................................... 16

Aulacoseira italica (Ehrenberg) Simonsen ............................................................................................ 16

Aulacoseira laevissima (Grunow) Krammer ....................................................................................... 40

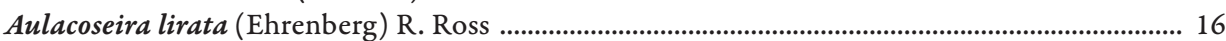

Aulacoseira nivalis (W. Smith) English et Potapova ..................................................................... 42

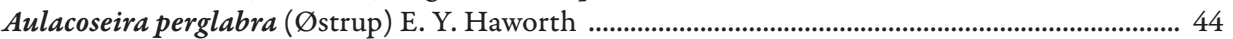

Aulacoseira pfaffiana (Reinsch) Krammer ............................................................................................ 46

Aulacoseira subarctica (Otto Müller) E. Y. Haworth ............................................................................. 16

Aulacoseira valida (Grunow) Krammer .............................................................................................. 48

Boreozonacola hustedtii Lange-Bertalot, Kulikovskiy et Witkowski ................................................ 50

Brachysira brébissonii R. Ross .......................................................................................................... 52

Caloneis silicula (Ehrenberg) Cleve .................................................................................................. 16 
Cavinula cocconeiformis (W. Gregory ex Greville) D. G. Mann et A. J. Stickle ................................ 16

Cavinula pseudoscutiformis (Hustedt) D. G. Mann et A. J. Stickle ..................................................... 54

Chamaepinnularia mediocris (Krasske) Lange-Bertalot et Krammer ............................................... 16

Chamaepinnularia soehrensis (Krasske) Lange-Bertalot et Krammer .............................................. 16

Cocconeis disculus (Schumann) Cleve .................................................................................................. 16

Cocconeis placentula var. euglypta (Ehrenberg) Grunow ...................................................................... 17

Cocconeis placentula var. intermedia (Héribaud-Joseph et M. Peragallo) Cleve ............................. 17

Craticula cf. submolesta (Hustedt) Lange-Bertalot ............................................................................ 56

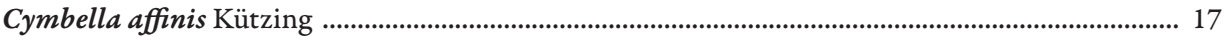

Cymbella aspera (Ehrenberg) Cleve ................................................................................................... 17

Cymbella bistritzae Oltean et Zanoschi ............................................................................................... 17

Cymbella helvetica var. punctata Hustedt .......................................................................................... 17

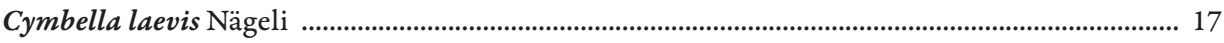

Cymbella neocistula Krammer ....................................................................................................... 17

Cymbella ventricosa var. hankensis Skv. .............................................................................................. 17

Cymbella ventricosa var. ovata Grunow ............................................................................................... 17

Cymbopleura amphicephala (Nägeli) Krammer ................................................................................. 17

Cymbopleura apiculata Krammer .................................................................................................. 58

Cymbopleura cuspidata (Kützing) Krammer ............................................................................. 17

Cymbopleura naviculiformis (Auerswald ex Heiberg) Krammer ........................................................ 60

Diadesmis contenta var. biceps (Grunow) P. B. Hamilton ...................................................................... 18

Diatoma ehrenbergii Kützing ......................................................................................................... 18

Diatoma ebrenbergii f. capitulata (Grunow) Lange-Bertalot .......................................................... 18

Diatoma hyemalis (Roth) Heiberg ........................................................................................... 18

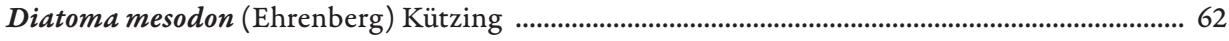

Diatoma vulgaris Bory ..................................................................................................................... 18

Diatoma vulgaris var. linearis Grunow ........................................................................................... 18

Diatoma vulgaris var. producta Grunow ........................................................................................ 18

Diploneis oblongella (Nägeli ex Kützing) Cleve-Euler ......................................................................... 18

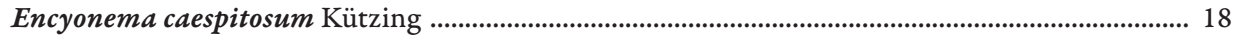

Encyonema elginense (Krammer) D. G. Mann …………............................................................... 18

Encyonema gaeumannii (Meister) Krammer ..................................................................................... 64

Encyonema hebridicum Grunow ex Cleve ...................................................................................... 18

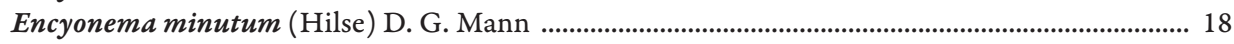

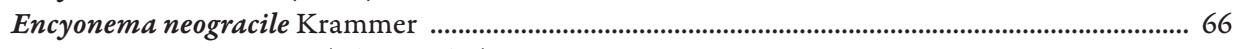

Encyonema perpusillum (Cleve-Euler) D. G. Mann ............................................................................ 68

Encyonema prostratum (Berkeley) Kützing ........................................................................................ 19

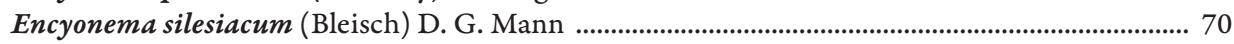

Encyonema ventricosum (C. Agardh) Grunow ........................................................................ 19

Encyonopsis cesatii (Rabenhorst) Krammer .............................................................................. 19

Encyonopsis microcephala (Grunow) Krammer .................................................................................. 19

Epithemia adnata (Kützing) Brébisson ............................................................................................ 19

Fragilaria brevistriata Grunow .................................................................................................. 19

Fragilaria capucina Desmazières ....................................................................................................... 19

Fragilaria capucina subsp. rumpens (Kützing) Lange-Bertalot .................................................... 19

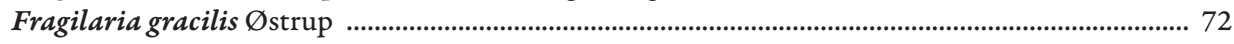

Fragilariforma bicapitata (Mayer) D. M. Williams et Round .......................................................... 74

Fragilariforma virescens (Ralfs) D. M. Williams et Round .............................................................. 76 
Fragilariforma virescens var. mesolepta (Rabenhorst) N. A. Andresen, Stoermer et R. G.

Kreis, Jr.

Frustulia crassinervia (Brébisson ex W. Smith) Lange-Bertalot et Krammer ................................ 78

Frustulia rhomboides (Ehrenberg) De Toni .................................................................................. 20

Frustulia vulgaris (Thwaites) De Toni .......................................................................................................... 20

Geissleria decussis (Østrup) Lange-Bertalot et Metzeltin .................................................................. 20

Geissleria cf. schoenfeldii (Hustedt) Lange-Bertalot et Metzeltin .................................................... 80

Genkalia boreoalpina A. Wojtal, C. E. Wetzel, L. Ector, N. Ognjanova-Rumenova et K. Buczkó .... 82

Genkalia digitulus (Hustedt) Lange-Bertalot et M. S. Kulikovskiy .................................................. 84

Genkalia subprocera (Hustedt) A. Wojtal, L. Ector, C. E. Wetzel, N. Ognjanova-Rumenova et K. Buczkó

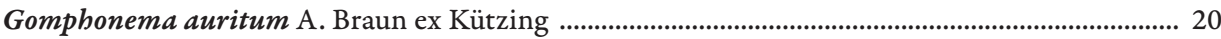

Gomphonema clavatum Ehrenberg ................................................................................................... 20

Gomphonema exilissimum (Grunow) Lange-Bertalot et E. Reichardt ......................................... 20

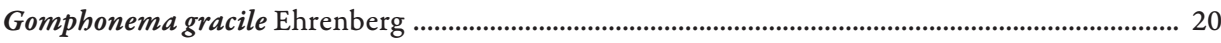

Gomphonema bebridense W. Gregory .......................................................................................... 20

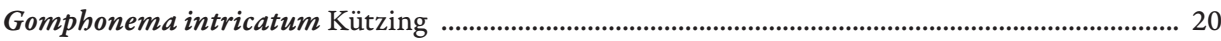

Gomphonema olivaceum (Hornemann) Brébisson ........................................................................... 20

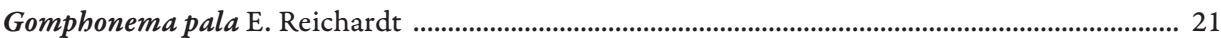

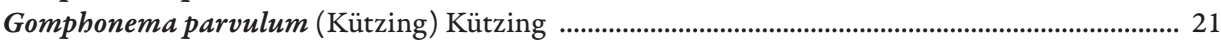

Gomphonema pumilum (Grunow) E. Reichardt et Lange-Bertalot ............................................. 21

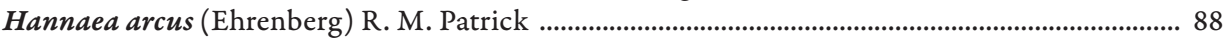

Hannaea linearis (Holmboe) Alvarez-Blanco et S. Blanco ......................................................... 2 21

Hantzschia amphioxys (Ehrenberg) Grunow ............................................................................. 21

Humidophila contenta (Grunow) Lowe, Kociolek, J. R. Johansen, Van de Vijver, Lange-Bertalot et Kopálová ............................................................................................................................................ 21

Humidophila fukushimae (Lange-Bertalot, M. Werum et A. Broszinski ....................................... 90

Humidophila perpusilla (Grunow) Lowe, Kociolek, Johansen, Van de Vijver, Lange-Bertalot et Kopálová ............................................................................................................................................ 21

Humidophila schmassmannii (Hustedt) K. Buczkó et A. Wojtal ..................................................... 94

Humidophila sp. ............................................................................................................................................... 92

Kobayasiella parasubtilissima (H. Kobayasi et T. Nagumo) Lange-Bertalot ............................... 21

Kobayasiella subtilissima (Cleve) Lange-Bertalot ........................................................................ 22

Kurtkrammeria neoamphioxys (Krammer) L. Bahls ................................................................. 22

Lindavia ocellata (Pantocsek) T. Nakov .......................................................................................... 22

Luticola mutica (Kützing) D. G. Mann ........................................................................................ 22

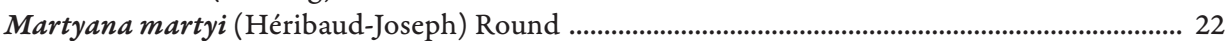

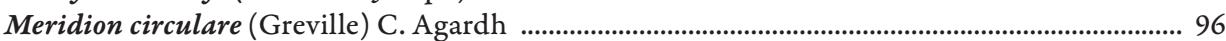

Meridion circulare var. constrictum (Ralfs) Van Heurck ............................................................... 98

Microcostatus krasskei (Hustedt) Johansen et Sray .................................................................... 100

Microfissurata paludosa Cantonati et Lange-Bertalot ................................................................ 102

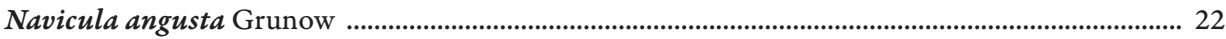

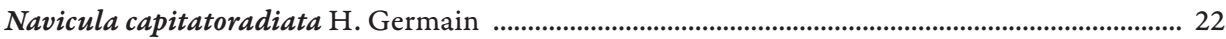

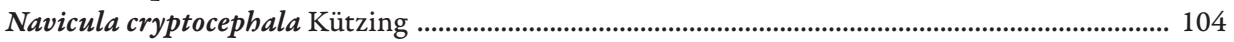

Navicula cryptotenella Lange-Bertalot ..................................................................................................... 22

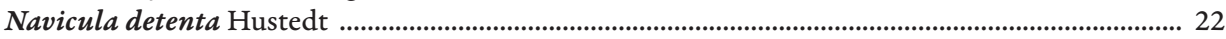

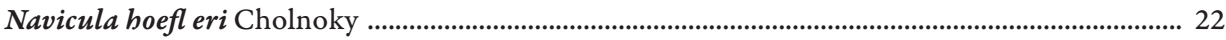

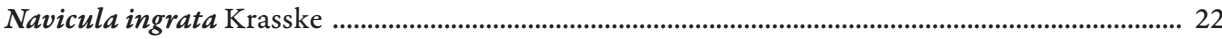




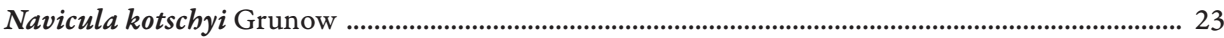

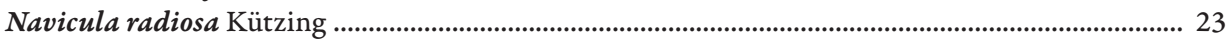

Navicula rhynchocephala Kützing ............................................................................................... 106

Neidium affine (Ehrenberg) Pfitzer ................................................................................................ 23

Neidium affine var. longiceps (W. Gregory) Cleve ....................................................................... 108

Neidium alpinum Hustedt ........................................................................................................... 110

Neidium amphigomphus (Ehrenberg) Pfitzer ................................................................................... 112

Neidium ampliatum (Ehrenberg) Krammer .............................................................................. 114

Neidium cf. antarcticum P. B. Hamilton, M. de Haan, K. Kopalova, R. Zidarova et

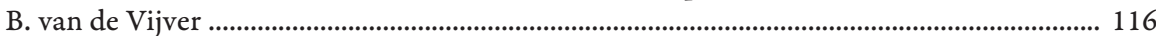

Neidium bisulcatum (Lagerstedt) Cleve ..................................................................................... 118

Neidium bisulcatum var. subundulatum (Grunow) Reimer ........................................................... 23

Neidium continentale M. S. Kulikovskiy, Lange-Bertalot et A. Witkowski .................................... 120

Neidium iridis (Ehrenberg) Cleve ................................................................................................... 122

Neidium productum (W. Smith) Cleve ………………………………………………................ 23

Nitzschia amphibia Grunow ............................................................................................................ 23

Nitzschia communis Rabenhorst ................................................................................................ 23

Nitzschia frustulum (Kützing) Grunow ............................................................................................ 23

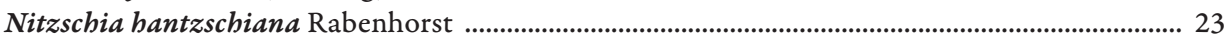

Nitzschia palea var. debilis (Kützing) Grunow ................................................................................ 23

Nitzschia perminuta (Grunow) M. Peragallo ........................................................................................ 124

Nupela fennica (Hustedt) Lange-Bertalot ............................................................................................. 24

Nupela impexiformis (Lange-Bertalot) Lange-Bertalot ................................................................. 126

Nupela lapidosa (Krasske) Lange-Bertalot ...................................................................................... 128

Nupela paludigena (R. P. Scherer) Lange-Bertalot .......................................................................... 130

Nupela pocsii K. Buczkó et A. Z. Wojtal .............................................................................................. 132

Nupela silvahercynia (Lange-Bertalot) Lange-Bertalot ...................................................................... 24

Nupela vitiosa (Schimanski) P. Siver et P. B. Hamilton ................................................................... 134

Orthoseira roeseana (Rabenhorst) O'Meara ........................................................................................ 136

Placoneis elginensis (W. Gregory) E. J. Cox ..................................................................................... 24

Planothidium distinctum (Messikommer) Lange-Bertalot ................................................................. 138

Planothidium frequentissimum (Lange-Bertalot) Lange-Bertalot ................................................... 24

Planothidium lanceolatum (Brébisson ex Kützing) Lange-Bertalot .............................................. 140

Planothidium oestrupii (A. Cleve) M. B. Edlund …………………………………........................... 24

Platessa conspicua (Ant. Mayer) Lange-Bertalot ............................................................................... 24

Psammothidium altaicum (Poretzky) Bukhtiyarova ......................................................................... 142

Psammothidium belveticum (Hustedt) L. N. Bukhtiyarova et Round ............................................ 144

Psammothidium helveticum var. minor (Flower et Jones) Buczkó, comb. nov. ............................. 146

Psammothidium kuelbsii (Lange-Bertalot) L. Bukhtiyarova et F. E. Round ................................... 148

Psammothidium lauenburgianaum (Hustedt) L. N. Bukhtiyarova et Round .................................. 24

Psammothidium levanderi (Hustedt) Bukhtiyarova et Round .......................................................... 150

Psammothidium marginulatum (Grunow) Bukhtiyarova et Round ............................................... 152

Psammothidium microscopicum (Cholnoky) S. Blanco ................................................................... 154

Psammothidium montanum (Krasske) S. Mayama ……………………………………..................... 25

Psammothidium rossii (Hustedt) L. Bukhtiyarova et Round ............................................................. 156

Psammothidium scoticum (R. J. Flower et V. J. Jones) Bukhtiyarova et Round ............................. 158

Psammothidium subatomoides (Hustedt) L. Bukhtiyarova et Round ............................................ 160

Pseudostaurosira parasitica (W. Smith) Morales ………………………………................................ 25 
Pseudostaurosira parasitica var. subconstricta (Grunow) E. Morales ............................................ 25

Pseudostaurosira pseudoconstruens (Marciniak) D. M. Williams et Round ................................ 162

Pseudostaurosiropsis E. A. Morales .......................................................................................................... 25

Reimeria sinuata (W. Gregory) Kociolek et Stoermer ....................................................................... 25

Rossithidium nodosum (Cleve) Aboal ......................................................................................................... 25

Sellaphora bacillum (Ehrenberg) Mann …................................................................................................. 25

Sellaphora elorantana (H. Lange-Bertalot) C. E. Wetzel ................................................................. 164

Sellaphora hentiensis (Kulikovskiy, Lange-Bertalot, A. Witkowski et N. I. Dorofeyuk) C. E.

Wetzel et L. Ector .................................................................................................................................. 25

Sellaphora laticeps (Hustedt) C. E. Wetzel, L. Ector, B. Van de Vijver, Compère et D. G. Mann .... 25

Sellaphora medioconvexa (Hustedt) Wetzel ................................................................................... 25

Sellaphora nigri (De Not.) C. E. Wetzel et L. Ector .......................................................................... 166

Sellaphora pupula (Kützing) Mereschkowsky ...............................................................................2 26

Sellaphora radiosa (Hustedt) H. Kobayasi .................................................................................... 26

Sellaphora rectangularis (W. Gregory) Lange-Bertalot et Metzeltin ............................................. 26

Sellaphora stauroneioides (Lange-Bertalot) J. Vesela et J. R. Johansen ......................................... 168

Sellaphora tridentula (Krasske) Wetzel ...................................................................................... 26

Stauroforma exiguiformis (Lange-Bertalot) R. J. Flower, V. J. Jones et F. E. Round ................... 170

Stauroneis acidoclinata Lange-Bertalot et Werum .......................................................................... 172

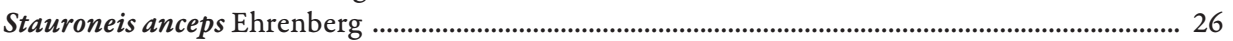

Stauroneis neofossilis Lange-Bertalot et Metzeltin ..................................................................... 174

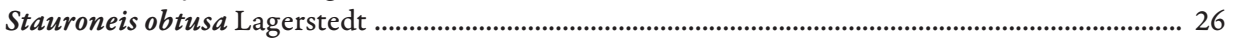

Stauroneis phoenicenteron (Nitzsch) Ehrenberg ................................................................................ 176

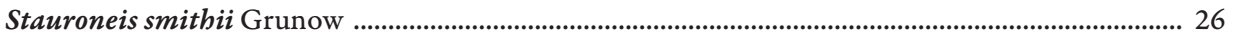

Stauroneis thermicola (Petersen) Lund ....................................................................................... 26

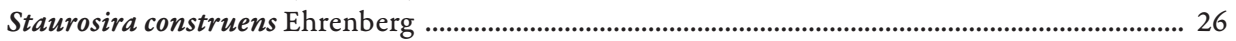

Staurosira construens var. binodis (Ehrenberg) Hamilton ................................................................. 26

Staurosira parasitoides Lange-Bertalot, Schmidt et Klee .................................................................... 27

Staurosira venter (Ehrenberg) Cleve and J. D. Möller ....................................................................... 178

Staurosirella leptostauron (Ehrenberg) D. M. Williams et Round .................................................... 27

Staurosirella pinnata (Ehrenberg) D. M. Williams et Round ...................................................... 180

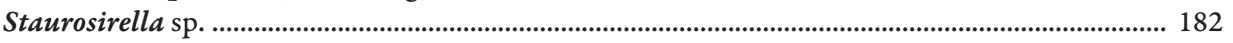

Stenopterobia delicatissima (F. W. Lewis) Brébisson ex Van Heurck ............................................. 184

Stephanodiscus astraea (Kützing) Grunow ................................................................................ 27

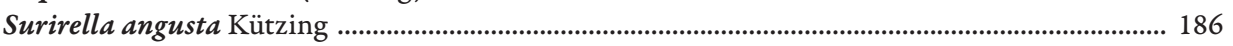

Surirella bifrons Ehrenberg ................................................................................................... 186

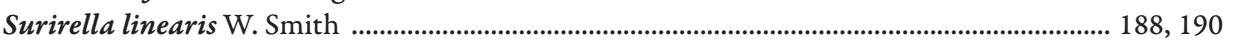

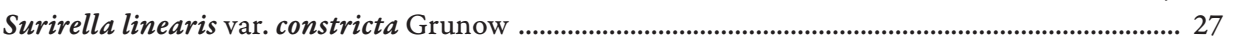

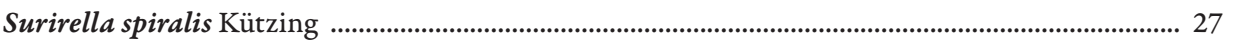

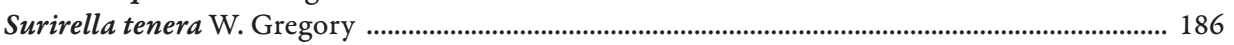

Tabellaria fenestrata (Lyngbye) Kützing ...................................................................................... 27

Tabellaria flocculosa (Roth) Kützing ........................................................................................... 192

Tetracyclus rupestris (Kützing) Grunow ................................................................................................... 27

Ulnaria ulna var. aequalis (Kützing) Aboal ........................................................................................ 27

Ulnaria ulna var. amphirhynchus (Ehrenberg) Aboal ................................................................ 27 